\title{
Topics in ultra-cold Bose gases: The Bose-Hubbard model; Analogue models for an expanding universe and for an acoustic black hole
}

Piyush Jain

Thesis submitted in partial fulfillment

of the requirements for the degree of Doctor of Philosophy at the Victoria University of Wellington.

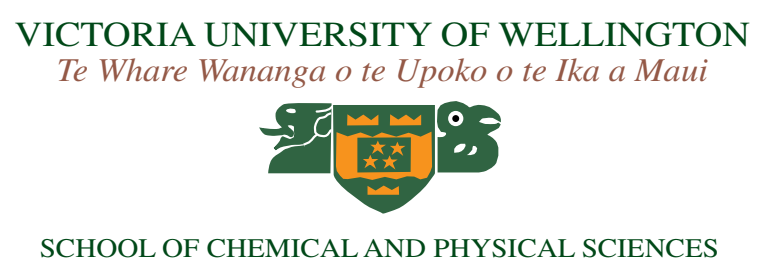

January 2007 


\section{REVISION HISTORY:}

Version 0.1, 6 February 2006.

Version 0.2, 14 February 2006.

Version 0.3, 14 August 2006.

Version 0.4, 5 October 2006.

Version 0.5, 2 January 2007.

Version 1.0, 9 January 2007 (submitted for examination).

Version 1.1, 16 August 2007 (including corrections). 


\begin{abstract}
In this thesis we consider the application of phase-space methods to Bose-Einstein condensates; the work comprises of three main parts: Part I: A phase-space method for the Bose-Hubbard model; Part II: An analogue model of an expanding universe in Bose-Einstein condensates. and Part III: An analogue model of an acoustic Black Hole in Bose-Einstein condensates.

In part I we present a phase-space method for the Bose-Hubbard model based on the Qfunction representation. In particular, we consider two model Hamiltonians in the mean-field approximation; the first is the standard "one site" model where quantum tunneling is approximated entirely using mean-field terms; the second "two site" model explicitly includes tunneling between two adjacent sites while treating tunneling with other neighbouring sites using the meanfield approximation. The ground state is determined by minimising the classical energy functional subject to quantum mechanical constraints, which take the form of uncertainty relations. For each model Hamiltonian we compare the ground state results from the Q-function method with the exact numerical solution. The results from the Q-function method, which are easy to compute, give a good qualitative description of the main features of the Bose-Hubbard model including the superfluid to Mott insulator. We find the quantum mechanical constraints dominate the problem and show there are some limitations of the method particularly in the weak lattice regime.
\end{abstract}

Analogue models of gravity have been motivated by the possibility of investigating phenomena not readily accessible in their cosmological counterparts. In particular, the prediction of quasiparticle creation in ultra-cold Bose gases in specific configurations can be viewed as an analogue to either cosmological particle creation or the Hawking effect.

In part II of this thesis we investigate the analogue of cosmological particle creation in an expanding universe by numerically simulating a Bose-Einstein condensate with a time-dependent scattering length. In particular, we simulate a $2 \mathrm{D}$ homogeneous condensate using the classical field method via the truncated Wigner approximation. We show that for several different expansion scenarios the calculated particle production is consistent with the underlying theory. For inflationary models we find the particle production for long wavelength modes coincides with the analytic theory within the acoustic approximation, whereas the particle production is suppressed for short wavelength (ie. free-particle like) modes. Moreover, particle production is enhanced for faster expansions, approaching the analytic result for the sudden expansion in the limit of a very fast expansion. For the case of a cyclic expansion, particle production peaks for a mode frequency that is approximately half of the driving frequency as expected for parametric resonance.

In part III of this thesis we investigate an acoustic black hole in a Bose-Einstein condensate, formed by two de Laval nozzles in a ring configuration - a system we refer to as the quantum de Laval nozzle. Our model is formulated in one dimension with a sinusoidal potential. For nonzero superfluid flow, this system can exhibit stable transonic flow with both black and white hole sonic horizons. Stationary states are found by solving the time-independent Gross-Pitaevskii equa- 
tion subject to a phase quantisation constraint. By solving the projected Bogoliubov-de Gennes equations for the system, we also find the discrete spectrum and quasiparticle modes. There are dynamical instabilities for certain values of winding number and potential depth, for which it is possible to construct pairs of normalisable modes. We further investigate the dynamics of the system using a classical field method based on the truncated Wigner approximation. For a low winding number and unstable configuration, we find exponential growth for the pair of unstable modes, whereas there is no growth in these modes for a stable configuration. This can be interpreted as non-degenerate parametric amplification, valid for short times. In contrast, for a large winding number, there is significant growth in modes for both stable and unstable configurations. This is indicative of higher order processes neglected in the quasiparticle picture, which is further reinforced by that fact that large winding number solutions require large nonlinearities. Finally, we consider the connection of our results with the usual semi-classical prediction of the Hawking effect. For an unstable configuration, the normalised unstable modes couple equal and opposite real frequencies, so that the growth in these modes represents the closest analogy with the Hawking effect for our quantum system. 


\section{Acknowledgements}

Completing a $\mathrm{PhD}$ has been one of the more challenging and rewarding experiences of my life, and I would like to take this opportunity to thank the many people who have helped and encouraged me along the way. (Although a single page seems insufficient for this purpose, I will do my best!)

First and foremost, I would like to thank Prof. Crispin Gardiner, my primary advisor, for his guidance and encouragement. He has always been very approachable and his diligence, intellect and good humour have made this a rewarding and enjoyable experience. I have been particularly grateful to him for striking just the right balance between providing guidance and allowing me to work independently. Moreover, I would like to thank him for continuing to advise me (and indeed helping with my transfer to Otago) after his shift from VUW to Otago, and therefore after his official obligations had expired.

To Dr. Ashton Bradley, my office mate for two years, thanks for all your help and friendship (and very frequent coffee breaks); our collaboration on Part III of this thesis was very enjoyable and productive. Thanks also for your superb proof-reading skills and constant encouragement. I am certainly in your debt (you deserve a whole page to yourself, but you're not getting one!).

To Silke Weinfurtner and Prof. Matt Visser, thank you for introducing me to analogue models of gravity, and in particular, for our fruitful collaboration on Part II of this thesis. Silke, thanks also for being a wonderful friend - expect a visit in Canada. Thanks also for your help with proof-reading.

To the staff and students at the School of Physical and Chemical Sciences at Victoria University, thank you to all the support I received over the years I spent there. Special mention to Prof. John Lekner (my secondary advisor), as well as Rob Lenihan, who certainly made life easier maintaining our assortment of computers.

To the staff and students at the Physics Department at the University of Otago, thank you for hosting me during the final stages of my study. In particular, thanks to Prof. Rob Ballagh and Dr. Blair Blakie for including me in the ultra-cold atoms group, as well as to the IT guys (Terry, Peter and Peter). Thanks also to Francesco who was a very welcome office mate for 6 months.

On a personal note, I would like to thank the many great friends and flatmates who have kept me sane (a matter of opinion!) during my studies, not least being: Rachel M, Nicky J, Fenella, Julia, Hamish, Bex, Rachel B and Chris. To Kathryn, thanks for your encouragement and love. Finally I would like to thank my family, especially my mother, who has always been supportive of me, even though I've managed to delay getting a real job for so long.

This work was supported by the Victoria University of Wellington Scholarships Office and the New Zealand Foundation for Research, Science and Technology Scholarship TAD-1054, and by the New Zealand Marsden Fund under contract numbers PVT-202 and UOO-0509. 



\section{Contents}

Abstract iii

$\begin{array}{lll}\text { Acknowledgements } & \text { v }\end{array}$

$\begin{array}{lc}\text { List of Acronyms } & \text { xiii }\end{array}$

1 Introduction 1

1.1 Bose-Einstein condensation . . . . . . . . . . . . . . . . 1

1.1.1 Historical development . . . . . . . . . . . . . . . . . 2

1.1.2 Advances in experiments and theory . . . . . . . . . . . . 3

1.2 Effective quantum field theory . . . . . . . . . . . . . . . 3

1.2.1 Second-quantised field theory description . . . . . . . . . . . 3

1.2.2 Mean-field description . . . . . . . . . . . . . . . . . 4

1.3 Phase-space representations . . . . . . . . . . . . . . . 5

1.4 Classical field methods . . . . . . . . . . . . . . . . . . . . . 6

1.4.1 Phase space methods . . . . . . . . . . . . . . . 6

1.4.2 The Projected Gross-Pitaevskii equation . . . . . . . . . . . . 8

1.4.3 The Stochastic Gross-Pitaevskii equation . . . . . . . . . . . . . 8

1.5 Truncated Wigner approximation . . . . . . . . . . . . . . 8

1.6 Thesis outline . . . . . . . . . . . . . . . . . . 10

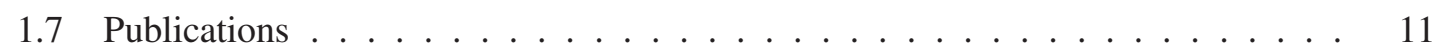

I A phase-space method for the Bose-Hubbard model 13

2 Introduction to the Bose-Hubbard Model 15

2.1 Introduction . . . . . . . . . . . . . . . . . . . . 15

2.2 Optical lattices . . . . . . . . . . . . . . . . . . . 16

2.3 The Bose-Hubbard model . . . . . . . . . . . . . . . . . . . . 16

2.3.1 Superfluid to Mott insulator transition . . . . . . . . . . . . . . . 17

2.3.2 Ground state solutions . . . . . . . . . . . . . . . 18

2.3.3 Quantum phase diagram . . . . . . . . . . . . . . 20

2.3 .4 Extensions to model . . . . . . . . . . . . . . . . . . . . . 21

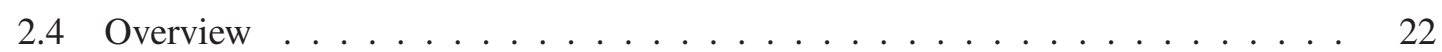


3 One site formulation $\quad 25$

3.1 Q-functions for one site states . . . . . . . . . . . . . . 25

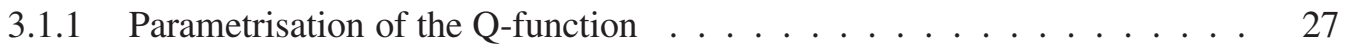

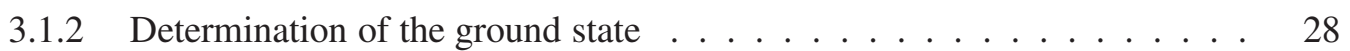

3.2 Exact numerical minimisation of one site problem . . . . . . . . . . 30

3.2.1 Formulation using one site states . . . . . . . . . . . . . . . . 31

3.2 .2 Numerical Solutions . . . . . . . . . . . . . . . . . . . . . . 32

3.2.3 Calculating the phase transition boundary . . . . . . . . . . . . 34

4 Two site formulation $\quad 37$

4.1 Q-function parameterisation on two sites . . . . . . . . . . . . . . . 37

4.1 .1 Constraints . . . . . . . . . . . . . . . . 38

4.1 .2 The ground state solution . . . . . . . . . . . . . . . 42

4.1 .3 Results . . . . . . . . . . . . . . . . . . 42

4.2 Exact numerical minimisation of two site problem . . . . . . . . . . . . . . . . . . . . 42

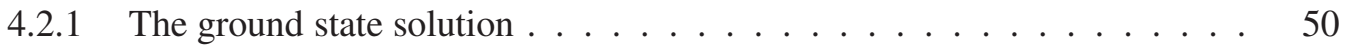

4.2 .2 Results . . . . . . . . . . . . . . . . . . 51

4.2.3 Calculating the phase transition boundary . . . . . . . . . . 52

4.2 .4 Comparison to other work . . . . . . . . . . . . . . . . . 54

4.3 Conclusions . . . . . . . . . . . . . . . . . . . . . 54

II An analogue model of an expanding universe in BECs 57

$5 \quad$ Analogue models of gravity 59

5.1 Introduction . . . . . . . . . . . . . . . . . . . . . 59

5.2 Emergent spacetimes in Bose-Einstein condensates . . . . . . . . . . . . . 60

5.2 .1 Establishing the analogy . . . . . . . . . . . . . . . . 60

5.3 Models of Analogue Gravity . . . . . . . . . . . . . . . . . . . . . 67

5.3.1 Cosmological Models . . . . . . . . . . . . . . . . . . . 67

5.3.2 Acoustic Black Hole Models . . . . . . . . . . . . . . . . . . . . . 68

5.3.3 Classical and Quantum systems _. . . . . . . . . . . . . . 70

5.4 Systems for Analogue Gravity . . . . . . . . . . . . . . . . 71

5.4 .1 Superfluid Helium . . . . . . . . . . . . . . . . . 71

5.4 .2 Shallow water waves . . . . . . . . . . . . . 71

5.4 .3 Slow light in dielectrics . . . . . . . . . . . . . . . 71

5.4.4 Bose-Einstein condensates . . . . . . . . . . . . . . . . . 72

5.5 The Hawking Effect . . . . . . . . . . . . . . . . . . . . . . . 73

5.6 Modifications to the analogy . . . . . . . . . . . . . . . 74

5.6.1 Extensions to theory of Hawking Radiation . . . . . . . . . . . . . . 74

5.6.2 Relevance to analogue models in BECs . . . . . . . . . . . . 76

5.7 Motivation and outline . . . . . . . . . . . . . . . . . . . . 77 
6 Expanding universe models in Bose-Einstein condensates 79

6.1 Introduction . . . . . . . . . . . . . . . . . . . . . . 79

6.2 Emergent FRW Universe . . . . . . . . . . . . . . . . . . . 80

6.2.1 Two dimensional model . . . . . . . . . . . . . . . . . . 81

6.3 Field quantisation and particle production $\ldots \ldots \ldots . \ldots . \ldots 83$

6.3.1 Acoustic approximation . . . . . . . . . . . . . . 86

6.3 .2 Beyond the acoustic approximation $\ldots \ldots \ldots 88$

6.4 Connection with Bogoliubov Theory . . . . . . . . . . . . . . . . . . . . . . 89

6.4 .1 Bogoliubov Theory . . . . . . . . . . . . . . . . . . . 90 90

6.4.2 Bogoliubov modes — Minkowski spacetime . . . . . . . . . . . . . 93

6.4.3 Acoustic modes — Minkowski spacetime . . . . . . . . . . . . . 93

6.4.4 Quasiparticle production . . . . . . . . . . . . . . . . . . 94

6.5 Quasiparticle production in acoustic approximation . . . . . . . . . . . . 95

6.5.1 Sudden transition $(2+1$ dimensions $) \ldots \ldots \ldots 9 . \ldots \ldots$

6.5.2 tanh expansion $(2+1$ dimensions $) \ldots \ldots . \ldots . \ldots 96$

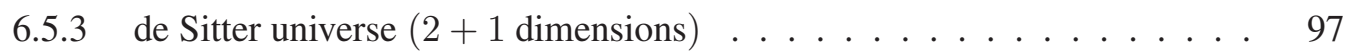

6.6 Quasiparticle production beyond the acoustic approximation . . . . . . . . . 102

6.6.1 Sudden Expansion . . . . . . . . . . . . . . . . . . . . . . 102

6.6.2 Cyclic universe $(2+1$ dimensions $) \ldots \ldots \ldots$. . . . . . . 103

7 The classical field method $\quad 105$

7.1 Introduction . . . . . . . . . . . . . . . . . . . 105

7.2 The truncated Wigner approximation . . . . . . . . . . . . . . . . 106

7.2 .1 Initial states: vacuum noise . . . . . . . . . . . . . . . . . . . 107

7.2 .2 Validity of the TWA _ . . . . . . . . . . . . . . 108

7.2 .3 Quasiparticle number . . . . . . . . . . . . . . . . 108

7.3 Numerical details . . . . . . . . . . . . . . . . . . . . . . . . . . . 109

7.3.1 Dimensionless GPE . . . . . . . . . . . . . . . . . . . . . 109

7.3.2 The projected RK4IP algorithm . . . . . . . . . . . . . . 110

7.4 Suitable parameter regime . . . . . . . . . . . . . . . . . . . . 110

7.4.1 Simulation parameters . . . . . . . . . . . . . . . 111

7.5 Estimating the temperature . . . . . . . . . . . . . . . . . . . 112

7.5.1 Ensemble averaging and ergodicity . . . . . . . . . . . . . 113

7.6 Power Spectral Density . . . . . . . . . . . . . . . . . . . . . . . . . . . . . . . 113

7.7 Results for Homogeneous BEC . . . . . . . . . . . . . . . . . . . . . . 115

7.7.1 Condensate depletion . . . . . . . . . . . . . . . . . 115

7.7.2 Temperature estimates for white noise vacuum . . . . . . . . . . 117

7.7 .3 Elementary excitations . . . . . . . . . . . . . . . 117

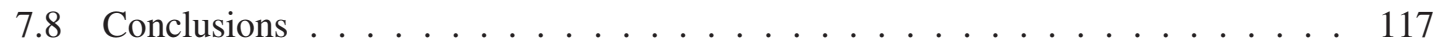

8 Expanding Universe simulations $\quad 119$

8.1 Introduction . . . . . . . . . . . . . . . . . . . . . . . . . . . . 119 
8.2 Quasiparticle production results . . . . . . . . . . . . . . . . . . . . . 119

8.2 .1 de Sitter universe . . . . . . . . . . . . . . . . . . . . . . . 119

8.2.2 Modified de Sitter expansion . . . . . . . . . . . . . . . . . 120

8.2 .3 tanh Expansion . . . . . . . . . . . . . . . . . . . . . 122

8.2 .4 Cyclic universe . . . . . . . . . . . . . . . . . 123

8.3 Discussion . . . . . . . . . . . . . . . . . . . . . . . . . . . . . . 124

8.3.1 Inflationary universe models . . . . . . . . . . . . . . . 124

8.3.2 Thermal equilibrium and the adiabatic regime . . . . . . . . . . 126

8.3 .3 Cyclic universe model . . . . . . . . . . . . . . . . . . . . 128

8.4 Conclusions . . . . . . . . . . . . . . . . . . . . . . . . . . . . . . . . . 129

8.4.1 Possible experimental implementation . . . . . . . . . . . . . . . . . . 129

8.4 .2 Outlook . . . . . . . . . . . . . . . . . . 130

III An analogue model of an acoustic Black Hole in BECs 133

9 The quantum de Laval nozzle $\quad \mathbf{1 3 5}$

9.1 Introduction . . . . . . . . . . . . . . . . . . . . . . . . . . . . 135

9.1 .1 Background . . . . . . . . . . . . . . . . 135

9.1.2 Hawking Radiation in analogue models . . . . . . . . . . . . . . 135

9.1 .3 Outline . . . . . . . . . . . . . . . . . . . . 137

9.2 The de Laval nozzle . . . . . . . . . . . . . . . . . . . . . . . . . 138

9.2 .1 Nozzle equation . . . . . . . . . . . . . . . . . . . 138

9.2 .2 The quantum de Laval nozzle . . . . . . . . . . . . . . . . . . 140

9.3 Estimated Hawking temperature ． . . . . . . . . . . . . . . . . . . . . . . 141

9.4 Stationary states . . . . . . . . . . . . . . . . . . . . . . . . 141

9.4.1 Equations of motion . . . . . . . . . . . . . . . . . . 142

9.4 .2 Ground state . . . . . . . . . . . . . . . . . . . 142

9.4.3 Solutions in hydrodynamic approximation . . . . . . . . . . . . . . 143

9.4.4 Dimensionless units . . . . . . . . . . . . . . . . . . . 146

9.4.5 Solving for the eigenstates . . . . . . . . . . . . . . . . . . 148

9.5 Stationary solution results . . . . . . . . . . . . . . . . . 150

9.6 Progress towards an experimental implementation . . . . . . . . . . . . . . . 152

9.6 .1 Toroidal geometries . . . . . . . . . . . . . . . . . 152

9.6 .2 Measurement techniques . . . . . . . . . . . . . . . . . . 153

10 Stability of the QdLN and the Hawking effect 155

10.1 Bogoliubov theory . . . . . . . . . . . . . . . . . . 155

10.1.1 The Bogoliubov-de Gennes equations . . . . . . . . . . . . . 155

10.2 Spectrum of the $\mathrm{QdLN} \ldots \ldots \ldots$. . . . . . . . . . . . . . 160

10.2.1 Numerical calculation of Bogoliubov modes . . . . . . . . . . 160

10.2 .2 Results . . . . . . . . . . . . . . . . . . . . . . 162 
10.3 Dynamics . . . . . . . . . . . . . . . . . . . . 166

10.3.1 Truncated Wigner approximation _ . . . . . . . . . . 166

10.3.2 Quasiparticle projection . . . . . . . . . . . . . . 167

10.3.3 Numerical details . . . . . . . . . . . . . . . . . . . . . . . 167

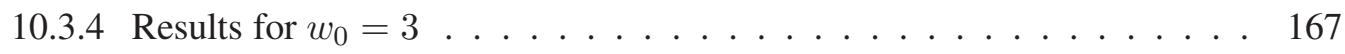

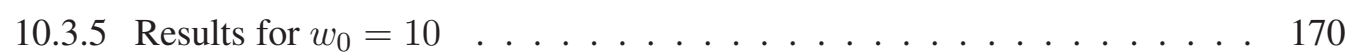

10.3 .6 Discussion . . . . . . . . . . . . . . . . . . 172

10.4 Connection with Hawking effect ． . . . . . . . . . . . . . . . . 174

10.4.1 Hawking effect in the QdLN . . . . . . . . . . . . . . . . . 174

10.4 .2 Open issues . . . . . . . . . . . . . . . . . . 175

10.4.3 Interpretation of Hawking effect for the QdLN . . . . . . . . . . 177

10.4.4 Limitations of classical field method . . . . . . . . . . . . . . . 178

10.5 Conclusions . . . . . . . . . . . . . . . . . . . . . . . . . 179

10.5.1 Summary . . . . . . . . . . . . . . . . . . . 179

10.5 Outlook . . . . . . . . . . . . . . . . 180

$\begin{array}{ll}\text { A Q-function for different states } & 181\end{array}$

A.1 Values of parameters in particular cases. . . . . . . . . . . . . . . . 181

A.2 A rigorous lower bound on the energy . . . . . . . . . . . . 183

B Numerical methods $\quad 185$

B.1 Projected Gross-Pitaevskii equation _ . . . . . . . . . . . . . . . . . . 185

B.2 Plane-wave basis . . . . . . . . . . . . . . . . . . . . . 186

B.3 Interaction picture . . . . . . . . . . . . . . . . . . . . 186

B.4 Projected RK4IP algorithm . . . . . . . . . . . . . . . . . . . . . . 187

B.5 Numerical grids . . . . . . . . . . . . . . . . . . . . . . . . . . . 188

B.6 Numerical stability and accuracy . . . . . . . . . . . . . . . . 188

B.6.1 Checking stability . . . . . . . . . . . . . . . . . . . 189

B.6.2 Time step . . . . . . . . . . . . . . . . . . . . . . . 189

$\begin{array}{ll}\text { C Bogoliubov-de Gennes equations for PGPE } & 191\end{array}$

$\begin{array}{ll}\text { List of Figures } & 194\end{array}$

$\begin{array}{ll}\text { References } & 203\end{array}$ 



\section{List of Acronyms}

BdG Bogoliubov-de Gennes (equations)

BEC Bose-Einstein Condensate

CFM Classical Field Method

DMRG Density Matrix Renormalisation Group

EFT Effective Field Theory

FRW Friedmann-Robertson-Walker (universe)

GPE Gross-Pitaevskii Equation

PGPE Projected Gross-Pitaevskii Equation

QdLN Quantum de Laval Nozzle

QFTCS Quantum Field Theory in Curved Spacetime

RK4IP Fourth-order Runge-Kutta in Interaction Picture (algorithm)

SDE Stochastic Differential Equation

TBR Three-Body Recombination

TWA Truncated Wigner Approximation

WKB Wentzel-Kramers-Brillouin (approximation) 



\section{Chapter 1}

\section{Introduction}

The initial observation of Bose-Einstein condensation in dilute atomic gases in 1995, seventy years after its prediction, has heralded in a new era in the study of many body quantum physics, that has brought with it new challenges - and opportunities. From an experimental point of view this has been accompanied by significant progress in the manipulation of ultra-cold atoms. It is now possible to prepare a Bose-Einstein condensate in a wide range of trap configurations, and even to tune the interactions between atoms. On the other hand, the fact that such systems are both weakly interacting and near zero temperature, means there is a simple theoretical description available in many regimes of interest. In particular, the mean-field Gross-Pitaevskii equation and extensions thereof, as well as the Bogoliubov theory of elementary excitations, provide a more than adequate description for many situations.

In this thesis, for the most part we will introduce theory as needed, but it is useful to give a brief overview of Bose-Einstein condensates and the theoretical methods for describing such systems. For more in-depth treatments, the reader is referred to the comprehensive review articles $[1,2]$ and texts $[3,4]$.

\subsection{Bose-Einstein condensation}

Bose-Einstein condensation is characterised by a macroscopic occupation of a single quantum state, typically the ground state of the system. This quantum degeneracy can only be achieved at very low temperatures where the de Broglie wavelength becomes comparable to the inter-particle spacing. In this regime, particles are no longer distinguishable and are necessarily subject to the rules of quantum statistics.

These rules arise from the fact that any physical observable must not depend on the interchange of two identical particles. Such an operation therefore introduces a global phase factor into the quantum many body wave function. Since exchanging the same particles again must reproduce the original wave function, the phase factor can only assume the values \pm 1 . Therefore all elementary particles fall into one of two classes: fermions, for which the wave function is anti-symmetric (-) and that obey Fermi-Dirac statistics; and bosons, for which the wave function is symmetric $(+)$ and that obey Bose-Einstein statistics. As it turns out, fermions obey the Pauli exclusion principle, which forbids any two particles from occupying the same quantum state. On the other hand, 
any number of bosons can occupy the same quantum state and it is this feature which underpins Bose-Einstein condensation.

A remarkable consequence of the spin-statistics theorem that emerges from relativistic field theories is that the exchange symmetry of identical particles is related to their intrinsic angular momentum or spin. Specifically, fermions have half-integer spin, whereas bosons have integer spin. In fact this picture is also valid for composite particles such as atoms, which can be treated as pointlike bosons/fermions depending on whether they have an even/odd number of constituent fermions. In this case the relevant physical processes should not be so energetic as to reveal the particle's internal structure.

\subsubsection{Historical development}

The phenomenon of Bose-Einstein Condensation was first predicted by Einstein in 1925 [5], by extending Bose's work on the statistics of photons [6], to massive particles. Einstein showed that below a certain temperature, a macroscopic fraction of the particles in an ideal Bose gas would condense into the single quantum state of lowest energy, this now being known as a Bose-Einstein condensate (BEC). In the derivation the phase transition was entirely due to quantum statistical effects, not requiring interactions between the particles. However, because the predicted transition temperature was so low, initially the idea of a BEC was relegated to a theoretical curiosity.

Following the discovery of superfluidity in liquid helium, London postulated in 1938 that Bose-Einstein condensation was the underlying mechanism for superfluidity in ${ }^{4} \mathrm{He}$ [7]. It has subsequently been shown that as superfluid Helium is a strongly interacting system, only a small fraction $(\sim 10 \%)$ of the fluid exists in the condensate phase, even at $T=0$. Therefore superfluid Helium is not ideally suited for the study of Bose-Einstein condensation in its original conception. The first microscopic theory of Bose-Einstein condensation was developed by Bogoliubov in 1947 for the case of a weakly interacting Bose gas [8]. Utilising the methods of second quantisation and assuming the system was highly condensed, Bogoliubov derived a general form of the excitation spectrum that included the effects of interactions. In 1956 Penrose and Onsager established a useful criterion for Bose-Einstein condensation, defining it as the emergence of off diagonal long range order in the one-particle density matrix [9]. In 1961 Gross [10] and Pitaevskii [11] independently derived an equation that describes the equation of motion for the condensate wave function (or mean-field order parameter). The Gross-Pitaevskii equation, as it is now known, is an indispensible tool for describing BECs near zero temperature.

However, it was only in 1995, following advances in laser cooling and magnetic trapping of atomic gases, that Bose-Einstein condensation was reported in trapped alkali gases by three separate groups, namely by the JILA group with ${ }^{87} \mathrm{Rb}$ atoms [12], the Rice group with ${ }^{7} \mathrm{Li}$ atoms [13] and the MIT group with ${ }^{23} \mathrm{Na}$ atoms [14]. Bose-Einstein condensation has subsequently been achieved in a number of other atomic species, notably in spin-polarised hydrogen [15], ${ }^{85} \mathrm{Rb}[16]$, metastable ${ }^{4} \mathrm{He}[17,18],{ }^{41} \mathrm{~K}[19]$ and ${ }^{133} \mathrm{Cs}[20]$. 


\subsubsection{Advances in experiments and theory}

With continued advances in both experiment and theory, the field of Bose-Einstein condensation has continued to develop rapidly, drawing on — and contributing to - many related disciplines. Some of the fascinating directions this research has taken include: quantum phase transitions in optical lattices [21], Bose-Einstein condensation of molecules (comprising pairs of fermionic atoms) [22], vortices and superfluidity [23], atom lasers [24], soliton waves [25], low dimensional systems [26] and proposals for analogue models of gravity [27].

\subsection{Effective quantum field theory}

The theoretical description of a BEC that is consistent with current experiments requires the inclusion of some features that do not appear in the textbook formulation for an ideal Bose gas, namely: interactions between atoms, and the effects of a trapping potential. The usual starting point for this description is the second-quantised formalism of quantum field theory.

\subsubsection{Second-quantised field theory description}

We review the effective quantum field theory for a BEC. In the second-quantised formalism, the effective Hamiltonian for a dilute Bose gas is:

$$
\hat{H}=\hat{H}_{0}+\hat{H}_{I}
$$

where the single particle Hamiltonian is

$$
\hat{H}_{0}=\int d \mathbf{x} \hat{\psi}^{\dagger}(\mathbf{x})\left[-\frac{\hbar^{2}}{2 m} \nabla^{2}+V_{\mathrm{ext}}(\mathbf{x})\right] \hat{\psi}(\mathbf{x})
$$

and the interaction Hamiltonian is

$$
\hat{H}_{I}=\frac{U}{2} \int d \mathbf{x} \hat{\psi}^{\dagger}(\mathbf{x}) \hat{\psi}^{\dagger}(\mathbf{x}) \hat{\psi}(\mathbf{x}) \hat{\psi}(\mathbf{x}) .
$$

$V_{\text {ext }}(\mathbf{x})$ is any external potential (eg. from a trap) and the two-body potential has been approximated via a contact potential by $U=4 \pi \hbar^{2} a / m$ in terms of the s-wave scattering length $a$, valid in the cold collision regime. Within this approximation the speed of sound is given in terms of the local condensate density $n=N / V$ by

$$
c=\sqrt{\frac{U n}{m}}
$$

The field operator $\hat{\psi}(\mathbf{x})$ annihilates a boson at position $\mathbf{x}$ and obeys the usual equal time commutation relations

$$
\begin{aligned}
{\left[\hat{\psi}(\mathbf{x}, t), \hat{\psi}\left(\mathbf{x}^{\prime}, t\right)\right] } & =\left[\hat{\psi}^{\dagger}(\mathbf{x}, t), \hat{\psi}^{\dagger}\left(\mathbf{x}^{\prime}, t\right)\right]=0 \\
{\left[\hat{\psi}(\mathbf{x}, t), \hat{\psi}^{\dagger}\left(\mathbf{x}^{\prime}, t\right)\right] } & =\delta\left(\mathbf{x}-\mathbf{x}^{\prime}\right) .
\end{aligned}
$$


The corresponding Heisenberg equation of motion for the field operator is

$$
\begin{aligned}
i \hbar \frac{\partial \hat{\psi}(\mathbf{x}, t)}{\partial t} & =[\hat{\psi}(\mathbf{x}, t), \hat{H}] \\
& =\left[-\frac{\hbar^{2}}{2 m} \nabla^{2}+V_{\text {ext }}(\mathbf{x})+U \hat{\psi}^{\dagger}(\mathbf{x}, t) \hat{\psi}(\mathbf{x}, t)\right] \hat{\psi}(\mathbf{x}, t) .
\end{aligned}
$$

Without further approximations, this equation cannot - using current computational resources - be solved for a realistic system since the Hilbert space becomes prohibitively large even for a system of just a few atoms.

\subsubsection{Mean-field description}

For the case of a weakly interacting Bose gas and for low temperatures $T \approx 0$, a very useful approximation arises from the fact almost all the atoms reside in a single quantum state, so the system occupies only a fraction of the available quantum states. In this case it is useful to write

$$
\hat{\psi}(\mathbf{x}, t)=\psi(\mathbf{x}, t)+\delta \hat{\varphi}(\mathbf{x}, t)
$$

where $\psi(\mathbf{x}, t)=\langle\hat{\psi}(\mathbf{x}, t)\rangle$ is a mean-field term (the condensate wave function) and $\delta \hat{\varphi}(\mathbf{x}, t)$ is that part of the quantum field associated with quantum and thermal fluctuations, with $\langle\delta \hat{\varphi}(\mathbf{x}, t)\rangle=0$. This is known as the Bogoliubov approximation.

\section{Gross-Pitaevskii equation}

This mean-field description itself may be treated with varying levels of approximation. In the very simplest approximation fluctuations are neglected altogether, The resulting equation, known as the Gross-Pitaevskii equation (GPE), is given by

$$
i \hbar \frac{\partial \psi(\mathbf{x}, t)}{\partial t}=\left[-\frac{\hbar^{2}}{2 m} \nabla^{2}+V_{\mathrm{ext}}(\mathbf{x})+U|\psi(\mathbf{x}, t)|^{2}\right] \psi(\mathbf{x}, t)
$$

This equation provides a description of the condensate in terms of a classical field, in much the same way that electromagnetic fields have a classical description in Maxwell's equations. The corresponding energy functional is

$$
E(\psi)=\int d \mathbf{x}\left(\frac{\hbar^{2}}{2 m}|\nabla \psi|^{2}+V_{\mathrm{ext}}(\mathbf{x})|\psi|^{2}+\frac{U}{2}|\psi|^{4}\right)
$$

where the first term is the kinetic energy of the condensate, the second term the potential energy due to the external trap, and the final term is the mean-field interaction energy.

\section{Bogoliubov theory}

While the GPE has been extremely successful for describing mean-field effects (ie. classical dynamics), it necessarily fails in the description of phenomena where quantum or thermal fluctuations play an important role. In fact, even at $T=0$, the condensate is depleted due to interactions 
so that some atoms reside in excited states. This can be seen explicitly by considering the theory of elementary excitations for a weakly interacting Bose gas, first formulated by Bogoliubov for the homogeneous case [8]. Here it was shown that the many body Hamiltonian can be diagonalised by an appropriately chosen canonical transformation of the particle operators. The resulting Hamiltonian represents a system of non-interacting quasiparticles, which exhibits a nonlinear dispersion relation for the quasiparticle excitations. This approximation is expected to be valid when the diluteness condition $n a^{3} \ll 1$ is satisfied, which is consistent with the result that the depletion is proportional to $\sqrt{n a^{3}}$ [28]. The formalism was subsequently extended to the case of an inhomogeneous case by Fetter [29]. An equivalent description is given by considering the linear response of the system to small oscillations around the stationary solution of the GPE, which leads to the so-called Bogoliubov-de Gennes (BdG) equations.

\section{Symmetry breaking}

The presence of a non-zero mean-field order parameter is associated with a breaking the U(1) gauge symmetry of the many body Hamiltonian (1.1). This is evident from the GPE by considering that the replacement of the field operator by its expectation value is the same as requiring $\hat{\psi}|\psi\rangle=$ $\psi|\psi\rangle$ which is the definition for a coherent state $^{1}$, a state with an indefinite number of particles (and a definite phase). The violation of the conservation of particle number is problematic in principle, and has motivated the development of number conserving Bogoliubov theories [31,32], which use the Penrose-Onsager criterion to define the order parameter.

\subsection{Phase-space representations}

An alternative route to a theoretical description of ultra-cold Bose gases, which has recently attracted much attention, is the use of phase-space representations, adopted from the field of quantum optics [30,33]. This has led to the development of methods for performing quantum dynamics that are able to go beyond the GPE description by using a multimode classical field that includes the condensate mode as well as noncondensate modes. We discuss these so-called classical field methods in the following section, but it is useful to first introduce phase-space representations.

The field of quantum optics leads to various quasi-classical distributions [30] which can be used to treat quantum processes using a c-number formalism. Specifically, these distributions arise from expressing the density operator of the system in terms of Glauber coherent states $|\alpha\rangle$. For a one-mode system with density operator $\hat{\rho}$, the most widely used of these are given by

Q-function: defined as

$$
Q\left(\alpha, \alpha^{*}\right)=\frac{1}{\pi}\langle\alpha|\hat{\rho}| \alpha\rangle
$$

and can be used to calculate anti-normally ordered moments

$$
\left\langle\hat{a}^{n} \hat{a}^{\dagger m}\right\rangle=\int d^{2} \alpha \alpha^{n} \alpha^{* m} Q\left(\alpha, \alpha^{*}\right) .
$$

\footnotetext{
${ }^{1} \mathrm{~A}$ discussion on coherent states can be found in [30].
} 
Because the Q-function always exists and is positive, it can be thought of as a quasiprobability distribution.

P-function: defined as

$$
\hat{\rho}=\int d^{2} \alpha P\left(\alpha, \alpha^{*}\right)|\alpha\rangle\langle\alpha|,
$$

and can be used to calculate normally ordered moments

$$
\left\langle\hat{a}^{\dagger n} \hat{a}^{m}\right\rangle=\int d^{2} \alpha \alpha^{* n} \alpha^{m} P\left(\alpha, \alpha^{*}\right) .
$$

However, it does not always exist as a positive well behaved function.

Wigner function: defined as

$$
W\left(\alpha, \alpha^{*}\right)=\frac{1}{\pi^{2}} \int d^{2} \lambda \exp \left(-\lambda \alpha^{*}+\lambda^{*} \alpha\right) \chi_{W}\left(\lambda, \lambda^{*}\right),
$$

where the characteristic function is

$$
\chi_{W}\left(\lambda, \lambda^{*}\right)=\operatorname{Tr}\left\{\hat{\rho} \exp \left(\lambda \hat{a}^{\dagger}-\lambda^{*} \hat{a}\right)\right\},
$$

and can be used to calculate symmetrically ordered moments

$$
\left\langle\left\{\hat{a}^{n} \hat{a}^{\dagger m}\right\}_{\mathrm{sym}}\right\rangle=\int d^{2} \alpha \alpha^{n} \alpha^{* m} W\left(\alpha, \alpha^{*}\right) .
$$

However, while it always exists, is not always positive.

The positive P-function: $P(\alpha, \beta)$, a P-function defined in a doubled phase-space, always exists and is positive, but practical calculations present some technical difficulties [30].

\subsection{Classical field methods}

The development of an effective quantum field theory for Bose gases that includes quantum fluctuations as well as finite temperature effects is a challenging goal. However, considerable progress has been made using so-called classical field methods, whereby the full quantum dynamics can be replaced with the dynamics of a multimode classical field under appropriate conditions. These methods are either based on heuristic arguments or rigorous phase space methods, or combinations thereof.

\subsubsection{Phase space methods}

The essential idea underlying these methods is as follows. The many body density operator for the system is expressed in terms of a coherent state basis using a classical quasiprobability distribution based on any of the phase space representations given in the previous section. By making use of well-known operator correspondences, it is then possible to map the master equation (or 
von Neumann equation) for the density operator to a partial differential equation (PDE) for the quasiprobability distribution. The resulting classical equation of motion is an exact mapping of the original problem, but is impractical to solve for multimode problems. However, it may be possible to put this equation in a Fokker-Planck form, and in this case the evolution of the system is entirely equivalent to solving a set of stochastic differential equations (SDE) for the phase space variables - ie. the quantum dynamics has been replaced the dynamics of a classical field. Quantum expectation values are found by calculating the corresponding moments using the appropriate quasiprobability distribution. In practice this requires taking averages over an ensemble of many trajectories of the classical field evolution. Significantly, the SDE includes the effects of quantum fluctuations in the initial state, which is sampled from the quasiprobability distribution, and/or by the presence of driving noise terms. Two of the more useful phase space methods for dynamics of Bose-Einstein condensates are:

\section{Truncated Wigner approximation}

Based on the Wigner phase space representation, the truncated Wigner approximation (TWA) was first applied to ultra-cold Bose gases by Steel et al. [34], and further developed by many others [35-40]. In this method, the equation of motion for the Wigner distribution is "truncated" by neglecting the third-order derivative terms, and the resulting Fokker-Planck equation corresponds to a set of SDEs that take the form of the GPE, but with initial states sampled from the Wigner distribution. This method has the obvious benefit that numerical methods developed for solving the GPE are also applicable. As the method is approximate, its validity has been the focus of some study [37,41]. The validity of the method requires the condition $N \gg M$ ( $N$ is the number of atoms in the condensate, and $M$ is the number of system modes). In its canonical form [34] the TWA is suited to systems near zero temperature, although it can be used for finite temperature treatments by sampling a thermal distribution for the initial state [35]. Recently, the TWA was successfully applied to the treatment of condensate collisions in order to describe the formation of s-wave scattering haloes $[41,42]$. In those studies, a projector was used so that the dynamical evolution was closed, only involving modes from a low-energy subspace of the system, which is consistent with the effective field theory. As we will make use of the TWA, we outline its derivation in the following section, and discuss its validity in section 7.2.

\section{Positive-P representation}

The positive-P representation was first applied to the dynamics of ultra-cold Bose gases by Steel et al. [34] and by Drummond and Corney [43]. This method leads to a Fokker-Planck equation for the evolution of the positive-P distribution, which is mapped formally to a set of SDEs with dynamical noise terms without requiring any approximation. Therefore the positive-P method can - in principle — be used to exactly simulate the quantum dynamics of the system. However, this method is prone to rather large divergences during evolution and is therefore valid only for short simulation times. These problems are accentuated in the absence of damping or when the system is strongly interacting. Recently, some progress has been made in this regard by the introduction of Gauge-P representations [44-46], and it 
remains a promising avenue of study.

\subsubsection{The Projected Gross-Pitaevskii equation}

The idea of using the GPE to simulate the dynamics of a finite temperature system was first proposed by Svistunov et al. [47-49], and was followed by a numerical study of evaporative cooling in a BEC that used this approach by Marshall et al. [50]. These ideas were further developed by Davis et al. [51-54] being formalised by the Projected Gross-Pitaevskii equation (PGPE). This formalism separates the system into two subspaces: a coherent region (low energy, highly occupied modes) and an incoherent region (high energy, sparsely occupied modes). Modes in the coherent region are treated by classical equations of motion, which is justified by neglecting the commutators of the field operators for the highly occupied modes. Neglecting the incoherent region entirely then leads to the PGPE, where a projector is introduced to ensure the evolution is closed in the low-energy subspace. The equilibrium properties of the system can be calculated from a single trajectory by the assumption of ergodicity. It should be emphasised that the PGPE and TWA methods are very closely related as both correspond to the evolution of a multimode classical field using the GPE. However, the interpretation of each method is distinct, and in practice this may lead to a difference in the choice of initial conditions or in the calculation of observables.

\subsubsection{The Stochastic Gross-Pitaevskii equation}

The formulation of the Stochastic Gross-Pitaevskii equation (SGPE) by Gardiner et al. [39, 5557], has been motivated by developing a finite temperature field theory for Bose gases to describe, for example, condensate growth and vortex nucleation. The method combines aspects of quantum kinetic theory (see [58] and references therein), as well as the TWA and PGPE formalisms. The essential idea is to separate the system into a condensate band (low-energy subspace for highly occupied modes) and a noncondensate band (high-energy subspace for thermal component). The coupling between the two bands is accounted for by the introduction of damping and noise terms. A related approach by Stoof [59] also leads to a stochastic form for the system evolution, but differs in some aspects such as the need to explicitly include the many body T-matrix for scattering processes.

\subsection{Truncated Wigner approximation}

In the work presented in this thesis (parts II and III), we will investigate the dynamics of a BEC near zero temperature, to which the truncated Wigner approximation is well suited. We therefore outline the derivation of this method here. For clarity of presentation we will follow the derivation of $[34,36,39,56]$, which uses the compact functional differentiation notation.

The classical field is defined by

$$
\psi(\mathbf{x}) \equiv \overline{\sum_{n}} \phi_{n}(\mathbf{x}) \alpha_{n}
$$


on a finite basis with wavefunctions $\phi_{n}(\mathbf{x})$ and phase space mode amplitudes $\alpha_{n}$. We further define the functional differentiation operators

$$
\begin{aligned}
\frac{\bar{\delta}}{\bar{\delta} \psi(\mathbf{x})} & \equiv \overline{\sum_{n}} \phi_{n}^{*}(\mathbf{x}) \frac{\partial}{\partial \alpha_{n}} \\
\overline{\bar{\delta}} & \equiv \overline{\sum_{n}} \phi_{n}(\mathbf{x}) \frac{\partial}{\partial \alpha_{n}^{*}}
\end{aligned}
$$

With the effective Hamiltonian (1.1), the equation of motion for density operator $\hat{\rho}$ (the von Neumann equation) is given by

$$
i \hbar \frac{\partial \hat{\rho}}{\partial t}=[\hat{H}, \hat{\rho}]
$$

With the above definitions, the following operator correspondences for the Wigner function can be found [30]:

$$
\begin{aligned}
& \hat{\psi} \hat{\rho} \leftrightarrow\left(\psi+\frac{1}{2} \frac{\bar{\delta}}{\bar{\delta} \psi(\mathbf{x})^{*}}\right) W\left(\alpha, \alpha^{*}\right), \\
& \hat{\psi}^{\dagger} \hat{\rho} \leftrightarrow\left(\psi^{*}-\frac{1}{2} \frac{\bar{\delta}}{\bar{\delta} \psi(\mathbf{x})}\right) W\left(\alpha, \alpha^{*}\right), \\
& \hat{\rho} \hat{\psi} \leftrightarrow\left(\psi-\frac{1}{2} \frac{\bar{\delta}}{\bar{\delta} \psi(\mathbf{x})^{*}}\right) W\left(\alpha, \alpha^{*}\right), \\
& \hat{\rho} \hat{\psi}^{\dagger} \leftrightarrow\left(\psi^{*}+\frac{1}{2} \frac{\bar{\delta}}{\bar{\delta} \psi(\mathbf{x})}\right) W\left(\alpha, \alpha^{*}\right) .
\end{aligned}
$$

Using these relations, equation (1.20) can be mapped to an equation of motion for the Wigner function

$$
\begin{aligned}
i \hbar \frac{\partial W}{\partial t}=\int d \mathbf{x}\{ & -\frac{\bar{\delta}}{\bar{\delta} \psi(\mathbf{x})}\left(-\frac{\hbar^{2}}{2 m} \nabla^{2}+V_{\text {ext }}(\mathbf{x})+U\left(|\psi(\mathbf{x})|^{2}-\delta_{R}(\mathbf{x}, \mathbf{x})\right)\right) \\
& \left.+\frac{U}{4} \frac{\bar{\delta}^{3}}{\bar{\delta}^{2} \psi(\mathbf{x}) \bar{\delta} \psi^{*}(\mathbf{x})} \psi(\mathbf{x})\right\} W-\text { c. c. }
\end{aligned}
$$

where the restricted delta function is defined as

$$
\delta_{R}\left(\mathbf{x}, \mathbf{x}^{\prime}\right) \equiv \overline{\sum_{n}} \phi_{n}^{*}\left(\mathbf{x}^{\prime}\right) \phi_{n}(\mathbf{x})
$$

Equation (1.25) contains a drift term (first order derivatives), and a "cubic noise" term (third order derivatives), but lacks any diffusion terms (second order derivatives), and so is often referred to as a generalised Fokker-Planck equation. The presence of third order derivative terms mean that (1.25) cannot be converted to an equivalent set of SDEs using well known methods [60] without further approximation. The standard approximation is to neglect (ie. "truncate") the third order terms so that the resulting stochastic differential equation is a Liouville equation, which in fact, takes exactly the same form as the GPE (1.8). However, there are three important differences here: 
(i) Quantum fluctuations are included in the initial state by sampling the the Wigner distribution this amounts to adding classical noise corresponding to half a particle per mode; (ii) The moments of the Wigner function give expectation values for symmetrically ordered operators, which are found by averaging over many trajectories in phase space; and (iii) A projector has been included by expressing the field (1.17) in a restricted basis, and in this case, the system dynamics are given by the PGPE (see for example $[39,41,56]$ where this has been formulated explicitly). We will further discuss the TWA, including its validity and implementation, in chapter 7.

\subsection{Thesis outline}

Two of the themes that have emerged from the field of ultra-cold Bose gases are: (A) Quantum phase transitions in highly correlated systems; and (B) Analogue models of gravity. This thesis consists of three theoretical studies, logically divided between the two themes as follows: Part I: A phase-space method for the Bose-Hubbard model (theme A); Part II: An analogue model of an expanding universe in Bose-Einstein condensates (theme B); and Part III: An analogue model of an acoustic Black Hole in Bose-Einstein condensates (theme B).

Although quite different in scope, all these studies share some important aspects with regards to the approach we take. In particular, in both cases we will make use of the effective quantum field theory for ultra-cold Bose gases, and moreover, use phase-space representations to map the quantum theory to an approximate c-number formalism, valid in some regime of interest.

\section{Part I: A phase-space method for the Bose-Hubbard model}

In part I of the thesis, we investigate the ground state phase diagram of the Bose-Hubbard model, which describes interacting bosons on an optical lattice. In chapter 1, we introduce the BoseHubbard model, with particular relevance to ultra-cold Bose gases and the superfluid to Mott insulator quantum phase transition. In chapter 3, we formulate the Bose-Hubbard model in terms of a phase-space method, based on the Q-function. This one-site model treats all hopping terms using the mean-field approximation. The energy function is reparameterised using the Q-function, and minimised subject to uncertainty relations to give the ground state phase diagram. These results are compared to the usual mean-field treatment, and we show the qualitative features of the phase transition are reproduced. In chapter 4, we introduce the two-site model, which explicitly includes correlations between two neighbouring sites while treating all other hopping terms with the mean-field approximation. Similarly to the one-site model, we find the ground state phase diagram using the Q-function reparameterisation, and compare these results with the exact numerical solutions to the same model Hamiltonian. We then compare our work with other investigations and conclude.

\section{Part II: An analogue model of an expanding universe in Bose-Einstein condensates}

In part II of the thesis, we investigate an analogue model of an expanding universe in a BEC. A simple model is formulated by considering a homogeneous condensate in two spatial dimensions 
with a time-dependent scattering length. The effective metric tensor for this system is that of a Friedmann-Robertson-Walker type universe, which is familiar from inflationary cosmology, and therefore leads to the notion of particle production.

In chapter 5, we introduce the concept of analogue models of gravity, with particular emphasis on models in Bose-Einstein condensates. In chapter 6, we develop our expanding universe model, and by using the acoustic approximation, can make analytic predictions for quasiparticle production using Bogoliubov's theory of linear excitations. Specifically we calculate the quasiparticle production for a sudden, tanh and de Sitter expansion scenarios. We also consider the question of particle production beyond the acoustic approximation, by including the so-called quantum pressure term. This leads to the idea of Lorentz violation, and should lead to a suppression of particle production for short wavelength modes. In chapter 7, we introduce the classical field method based on the truncated Wigner approximation. We discuss the requirements of the method, and suggest a suitable set of parameters with which to simulate our expanding universe model. In chapter 8 , we give results for the classical field method simulations of the expanding universe model. In particular we show results for inflationary scenarios with de Sitter and tanh expansions, and also for a cyclic universe model. We compare these results to the predictions made in chapter 6 , then then conclude by considering the experimental relevance of the results, and suggesting avenues for further work.

\section{Part III: An analogue model of an acoustic Black Hole in Bose-Einstein condensates}

Part III continues the theme started in part II. We present an analogue model of an acoustic black hole in a trapped Bose gas. The specific geometry is based on using two de Laval nozzles in a ring, which is implemented by a periodic potential. We refer to this configuration as the quantum de Laval nozzle.

In chapter 9, we introduce the quantum de Laval nozzle, and find its stationary solutions using the time-independent GPE. We further show that this system does indeed exhibit both a black and white hole horizon. In chapter 10, we solve the Bogoliubov-de Gennes equations for the system, and show the presence of dynamical instabilities for certain values of the potential amplitude and winding number. It is then possible to construct positive norm modes following the analysis of Leonhardt et al. [61], which are used to analyse the evolution of the system. Single trajectory dynamics, based on the truncated Wigner approximation, show that for a low winding number there is exponential growth in the dynamically unstable modes. This instability is associated with the Hawking effect, although the picture is not entirely clear for several reasons, namely: the constraint of phase quantisation, the discreteness of the spectrum, and dynamics for large winding numbers. We then conclude and suggest some possible extensions to this work.

\subsection{Publications}

The work from part I has been previously published [62], and is included here (in chapters 2-4) with some modifications necessary for consistency of presentation. The work from part II (in particular, from chapters 6 and 8) is currently in preparation for publication, as is the work from 
part III (chapters 9 and 10). 


\section{Part I}

\section{A phase-space method for the Bose-Hubbard model}





\section{Chapter 2}

\section{Introduction to the Bose-Hubbard Model}

\subsection{Introduction}

Quantum phase transitions in strongly correlated systems have recently attracted a lot of interest. This has been encouraged by two significant developments: Firstly, it is now possible to trap cold bosonic atoms in optical lattices. Secondly, the prediction [63] that a Bose-Hubbard Hamiltonian could be realised with this configuration, and therefore the possibility of observing the transition between superfluid and Mott insulator phases. Indeed this led to the subsequent development of an experiment in which these predictions were verified [64], and has lent urgency to the quest for a computationally simple description of the phenomena involved.

In experiments the transition from a superfluid to Mott insulator phase can be achieved by increasing the lattice strength. It is desirable to find a description which can cover both of the two regimes of qualitatively different behaviour: for a weak lattice where the atoms are delocalised ie. the superfluid phase; and for a strong lattice where the atoms are strongly localised and the system is strongly correlated - if additionally there is an integral number of atoms per site it is possible to enter the Mott insulator phase.

The dynamics of the Bose-Hubbard model in states for the strong lattice region presents formidable technical difficulties. The so-called Gutzwiller approximation $[63,65,66]$ has been used in several treatments, but because it represents the wavefunction as a product of wavefunctions at different sites, the description of intersite correlations is necessarily very approximate. Progress has been made $[67,68]$ in regions near to the weak lattice regime by using a selfconsistent Bogoliubov method, in which the statistics of the quantum fluctuations are treated by what amounts to a Gaussian ansatz. However, this method cannot be used near the strong lattice regime because in this case the statistics are far from Gaussian.

In this part of the thesis, we introduce an approximate phase space representation for the BoseHubbard Hamiltonian, based on the Q-function, which is applicable in both regimes of interest. By utilising the mean-field approximation, we are able to find the ground state solutions, and therefore determine the phase diagram. Before we embark on this programme, in the remainder of this chapter, we introduce the ideas of quantum phase transitions in optical lattices, and then give an overview of our work, which is presented in chapters 3 and 4 . 


\subsection{Optical lattices}

Light fields - such as those provided by lasers - have become a useful tool for the manipulation of atoms. In particular, an optical lattice is formed by the standing wave interference pattern of two or more laser beams. The resulting periodic potential constitutes an array of microtraps that may trap atoms in an artificial crystal structure. The trapping force is due to the ac-Stark shift, whereby the atoms experience an energy shift due to an induced dipole moment (see $[69,70]$ and references therein). For the resulting conservative potential, this must occur at laser wavelengths that are far-detuned from any atomic resonance. For a red detuning, neutral atoms experience a force in direction of increasing field - ie. they are attracted to the maxima of the laser field intensity. For a blue detuning the effect is reversed, with atoms attracted to the minima of the field. In one dimension for example, an optical lattice can be formed by two counter-propagating laser beams, with the lattice spacing equal to half the laser wavelength.

Experiments with BECs in optical lattices have led to the observation of highly correlated quantum states as described by the Bose-Hubbard model. In particular, approaching the Mott insulator state, Orzel et al. found evidence of squeezed states in a one-dimensional optical lattice [71]. This was soon followed by the landmark experiments by Greiner et al. in three-dimensional optical lattices that demonstrated the Superfluid to Mott insulator transition [64], and also the collapse and revival of the coherent matter wave by entering (and leaving) the Mott insulator state [72]. The experiment in [64] also showed evidence for the excitation gap that occurs for the Mott insulator state.

In general, the propagation of atoms in a periodic potential is represented by Bloch states that exhibit an energy band structure for the system (for localised states it is appropriate to use the Wannier function basis). For high occupation numbers, a multi-band Bose-Hubbard model is required to describe interactions between atoms in different energy bands [73]. However, the multi-band description complicates the theory of interacting atoms in an optical lattice, and so it is often desirable to consider only the lowest energy band - that is, the ground state of the optical lattice. Indeed, in the above experiments the condensate was loaded into the optical lattice adiabatically (ie. the lattice was turned on slowly) so that the system remained in the ground state. However, experiments with non-adiabatic loading have also been performed more recently [74].

\subsection{The Bose-Hubbard model}

The Bose-Hubbard model was first introduced by Fisher et al. to describe the superfluid to insulator phase transition in liquid ${ }^{4} \mathrm{He}$ [75], but is generally applicable to any system of interacting bosons on a lattice. It is possible to derive the Bose-Hubbard Hamiltonian by starting with the many body Hamiltonian (1.1), where the external potential includes components from the optical lattice and from any additional trapping potential $\left(V_{\mathrm{ext}}=V_{\text {lattice }}+V_{\text {trap }}\right)$. The simplest form of the model is given when the atoms are loaded adiabatically into the lattice, so that they remain in the lowest vibrational state (that is, the lowest energy band of the lattice). Using a Wannier function 
basis, the resulting Bose-Hubbard Hamiltonian is given by [63]

$$
H_{\mathrm{BH}}=-J \sum_{\langle i, j\rangle} a_{i}^{\dagger} a_{j}+\frac{1}{2} U \sum_{i} \hat{n}_{i}\left(\hat{n}_{i}-1\right)+\sum_{i} \epsilon_{i} \hat{n}_{i}
$$

where $a_{i}^{\dagger}$ and $a_{i}$ respectively are the creation and annihilation operators for a boson on the $i$ th site and $\hat{n}_{i} \equiv a_{i}^{\dagger} a_{i}$ is the corresponding number operator. The first term of the Hamiltonian describes the quantum tunneling ("hopping") between neighbouring sites with an amplitude $J$ which is only non-zero when $i$ and $j$ are adjacent $(\langle i, j\rangle$ indicates the summation only occurs over adjacent sites). The second term represents the on-site interaction energy which is taken as repulsive with $U \geq 0$, as is the case for Rubidium atoms. The final term arises from the presence of a trapping potential $V_{\text {trap }}$, which leads to an on-site energy shift $\epsilon_{i}$. Because the lattice spacing is typically much smaller than the scale on which the trapping potential varies, it is reasonable to neglect this term - which we will henceforth do - the main benefit being that the model becomes tractable.

As we will be formulating the model using the Q-function representation [30], and noting that this distribution is adapted to antinormal ordering, we write the Bose-Hubbard Hamiltonian above in the antinormal ordered form

$$
H_{\mathrm{BH}}=-J \sum_{\langle i, j\rangle} a_{i} a_{j}^{\dagger}+u \sum_{i} a_{i} a_{i} a_{i}^{\dagger} a_{i}^{\dagger}
$$

where in order to simplify notation we have set $u=U / 2$, and where we have neglected the on-site energy due to an additional trap. We note that this Hamiltonian is easily related to its normally ordered counterpart using the commutation relation $\left[a_{i}, a_{j}^{\dagger}\right]=\delta_{i j}$.

\subsubsection{Superfluid to Mott insulator transition}

The Bose-Hubbard Hamiltonian exhibits solutions for two qualitatively different regimes:

a) Weak Lattice $(u / J \approx 0)$, when the atoms are delocalised, and are thus highly mobile; the system is weakly correlated.

b) Strong Lattice $(J / u \approx 0)$, when the atoms are localised; the system is highly correlated. In this case there are two subcases

i) Commensurate, when the number of atoms per site is integral, and the state is a product of number states on each site. In this case there can be no mean-field.

ii) Incommensurate, when the number of atoms per site is non-integral, and in this case there can be a mean-field.

In the case of an integral average site filling, as the strength of the lattice varies, a transition from the delocalised to the localised situation takes place, and one passes from state with a non-zero mean-field to a state with no mean-field. Conventionally, if there is no mean-field, one speaks of a Mott insulator phase, while if there is a non-vanishing mean-field, the terminology superfluid 
phase is applied. At zero temperature, the "competition" between the tunneling energy $J$ and onsite interaction energy $u$ determines a phase boundary between the Mott insulator and superfluid phases - this is quantified by a critical value $(J / u)_{c}$.

The onset of the Mott insulator phase is also characterised by the existence of an energy gap, which represents the energy required to add a particle or hole excitation to the system. In the grand canonical ensemble description, this can be expressed by the condition that $\partial n / \partial \mu=0$ where $n$ is the mean atom number on a site, and $\mu$ is the chemical potential - the Mott insulator phase is then said to be incompressible.

The superfluid - Mott insulator transition is an example of a continuous quantum phase transition, which is governed by quantum fluctuations - significant near zero temperature. The phase transition is driven by changing some external parameter that appears in the system Hamiltonian, rather than a change of temperature, as is the case with classical phase transitions. Here the external parameter is the parameter $J / u$, which is easily tuned in experiments by increasing or decreasing the depth of the optical lattice potential.

The Heisenberg uncertainty relation between number and phase largely determines the nature of each quantum phase: In particular, the superfluid phase is characterised by large number fluctuations on each site with an associated reduction in phase fluctuations, which are features of a coherent state ${ }^{1}$. In contrast, the Mott insulator phase is characterised by a fixed number of atoms on each site and large fluctuations in the phase between different sites, which represents a Fock number state. In fact the transition to the Mott insulator phase is associated with the breaking of $\mathrm{U}(1)$ symmetry [76].

\subsubsection{Ground state solutions}

In practice it is difficult to find exact solutions to the Bose-Hubbard model since the required basis of states is unfeasibly large even for a modest lattice size [67]. In light of this, a number of approximation methods have been used, with varying success, to describe the zero-temperature phase diagram. We give an overview of these methods with particular emphasis on describing the superfluid to Mott insulator phase transition, which occurs at commensurate occupations.

A standard method of dealing with excitations in a homogeneous Bose gas, the Bogoliubov approximation, has recently been applied by several authors to the case of atoms trapped in an optical lattice $[67,68,77]$. However, while this method is appropriate for describing number squeezing in the superfluid phase $[67,77]$, it does not correctly predict the superfluid to Mott insulator transition [68]. This is because the Bogoliubov approach fails to account for the large depletion of the condensate that occurs in the immobile Mott insulator phase.

In contrast, Garcia-Ripoll et al. have shown the equivalence of the Bose-Hubbard and quantum rotor models in the limit of commensurate occupations and strong interactions [78]. Using a variational ansatz, these authors then showed the quantum rotor model is well suited to describing the Mott insulator state as well as the transition to the superfluid phase, even though it cannot

\footnotetext{
${ }^{1}$ This description is not complete however, since the total number operator $\hat{N}=\sum_{i} \hat{n}_{i}$ commutes with the Hamiltonian (2.1), and so the total atom number should be conserved - ie. the coherent state description is only an approximation. Strictly speaking, to find the superfluid order parameter one should calculate the superfluid fraction [75] instead of using the symmetry breaking mean-field order parameter.
} 
represent the superfluid phase itself.

A more successful approach utilises a mean-field approximation based on the Gutzwiller ansatz for bosons $[63,65,66]$ in which the many-body wavefunction of $N$ atoms occupying $L$ sites is given as a product of single-site states of the form

$$
|\psi\rangle=\prod_{l=1}^{L}\left(\sum_{n=0}^{\infty} c_{n}|n\rangle\right)
$$

Such a wavefunction can be used to describe a wide variety of states, and in particular, can represent both the superfluid and Mott insulator phases. In particular, in the limit where tunneling dominates $(u / J \approx 0)$, the system is in the superfluid state and the wavefunction is of the form

$$
\left|\psi_{\mathrm{SF}}\right\rangle \propto\left(\sum_{l=1}^{L} a_{i}^{\dagger}\right)^{N}|0\rangle
$$

This approximates a coherent state for large $N, L$ where the statistics are Poissonian. In the opposite limit where on-site interactions dominate $(J / u \approx 0)$ and in the case of commensurate occupation, the system is in the Mott insulator state. The wavefunction is then given by a product of Fock number states

$$
\left|\psi_{\mathrm{MI}}\right\rangle \propto \prod_{l=1}^{L}\left(a^{\dagger}\right)^{n}|0\rangle
$$

with an occupation of $n=N / L$ atoms per site. In this regime the number variation vanishes and the statistics are far from Gaussian.

Using the Gutzwiller approximation the tunneling term can be treated using a mean-field approximation $[63,66,68,76]$; this introduces a symmetry breaking term into the Hamiltonian that accounts explicitly for the presence of the superfluid to Mott insulator transition. A vanishing mean-field identifies the Mott insulating phase where intersite interactions are suppressed. For this reason the mean-field is analogous to the superfluid order parameter. The main advantage of the Gutzwiller ansatz is the approximation of the Bose-Hubbard Hamiltonian by the sum of singlesite Hamiltonians. However, this methodology necessarily approximates the intersite correlations and therefore fails to account correctly for the superfluid to Mott insulator transition boundary. As we shall show, a better approximation is to use the two-site ansatz which includes the correlations between two adjacent sites while still treating tunneling to other sites with the mean-field approximation.

The mean-field approximation has also been applied to the Bose-Hubbard model in a number of other scenarios including the variational principle $[63,75]$, time-dependent variational principle $[79,80]$ and using perturbative methods [81-85]. The latter case makes use of an important feature of the Bose-Hubbard model: the existence of an energy gap in the Mott insulating phase. In the superfluid phase this energy gap disappears so that the phase is compressible. The phase transition boundary can then be calculated by considering a perturbative expansion of the ground state energy around the particle/hole states.

The Bose-Hubbard model has also been investigated using exact numerical methods in small 


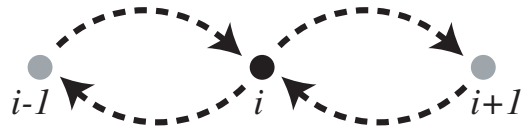

(a) One site model

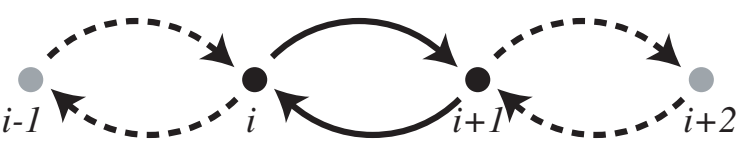

(b) Two site model

Figure 2.1: Treatment of hopping terms in the Bose-Hubbard model for a lattice of dimension $d=$ 1. The dashed arrows indicate those hopping terms $J a_{i} a_{j}^{\dagger}$ included by a mean-field approximation, whereas the solid arrows indicate those terms included explicitly in the Hamiltonian. We consider two formulations: (a) The hopping terms between the $i$ th site and its $z=2 d$ nearest neighbours are treated solely by a mean-field approximation; (b) The hopping terms between two adjacent sites $i$ and $i+1$ are included explicitly, whereas the hopping terms between each of these sites and and their (other) nearest $z^{\prime}=2 d-1$ neighbours are included using the mean-field approximation.

systems, with the Quantum Monte-Carlo (QMC) and Density Matrix Renormalisation Group (DMRG) techniques. In particular, QMC simulations have been used to study the phase diagram in both one [86-88] and two [89-91] dimensional lattices. More recently, this approach has been made more efficient by adapting the Bose-Hubbard model to a stochastic Gutzwiller ansatz [92].

The DMRG approach was first developed by White to deal with lattice systems [93] and has subsequently been applied to the one-dimensional Bose-Hubbard model with the infinite-system algorithm [78,94,95] and the more accurate finite-system algorithm [96,97]. This method benefits from truncation of the Hilbert space with the most probable states represented by the reduced density matrix. The size of the system is grown by adding lattice sites at each iteration. The method is numerically exact in that a desired accuracy can be achieved by varying the number of states retained at each iteration; it has therefore been used as a useful basis of comparison for the validity of other methods [78]. It should be noted that the standard formulation of DMRG is for one dimensional systems. The adaption of the algorithm to higher dimensions is problematic not least because it may require the representation of prohibitively large Hilbert spaces; therefore the DMRG has yet to be adapted to the Bose-Hubbard model in dimensions higher than one.

\subsubsection{Quantum phase diagram}

Using the mean-field approximation, the Hamiltonian can be decoupled into a sum of single site terms, and the ground state solutions are found by a straightforward calculation. For comparison with our following results, we now calculate the phase diagram resulting from this procedure.

\section{The mean-field approximation}

In the mean-field approximation, one assumes that the effect of the the nearest neighbours on the site $i$ is given by a c-number mean-field $\mathcal{E}=\left\langle a_{i}\right\rangle$ which, by a choice of phase, we take as real, and which is independent of $i$ for a homogeneous system. Thus we can write

$$
H_{\mathrm{BH}} \rightarrow H_{\mathrm{mf}}=\sum_{i}\left\{-\frac{Z}{2} \mathcal{E}\left(a_{i}^{\dagger}+a_{i}\right)+u a_{i} a_{i} a_{i}^{\dagger} a_{i}^{\dagger}\right\}
$$



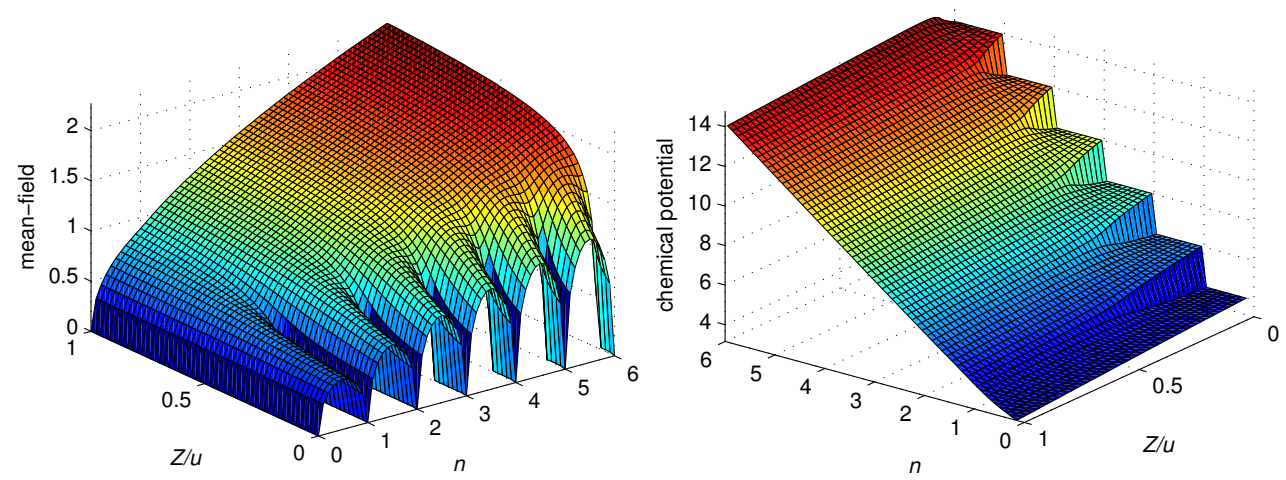

Figure 2.2: Ground state phase diagram for the Bose-Hubbard model using a self-consistent meanfield approach; the mean-field and chemical potential are both shown as a function of the relative interaction strength $Z / u$ and mean occupation number $n$ (normal ordered).

where $Z$ is proportional to the nearest neighbour value of $J$ multiplied by the number of nearest neighbours (see Figure 2.1a). As noted in Sachdev's book [98], to solve the system one finds the lowest eigenvalue of $H_{\mathrm{mf}}$ on a single site, for which the value of $\mathcal{E}$ must match that of $\left\langle a_{i}\right\rangle$.

Thus, one assumes a value for the mean-field, computes the ground state eigenfunctions of the Hamiltonian, whose mean-field should equal that initially assumed. Unlike the more conventional method, we do this at fixed mean occupation of the site, not fixed chemical potential, and compute the chemical potential from the energy. Since the solutions of this process are well known, it will provide an interesting testbed for the phase space method we wish to propose.

\section{Self-consistent mean-field results}

The results obtained by this procedure are shown in Figure 2.2. The usual features of the superfluid to Mott insulator transition are present. In particular, in the Mott insulator phase, the mean-field vanishes for commensurate occupations when the relative interaction strength $(Z / u)$ is below a critical value. There is a corresponding energy gap evident in the chemical potential which represents the energy required to add or remove a particle to the system; hence the Mott phase is incompressible. When the density is incommensurate or the tunneling dominates, there is a transition to the superfluid phase where the mean-field is non-zero.

\subsubsection{Extensions to model}

While the Bose-Hubbard Hamiltonian given by (2.2) encapsulates the essential physics of bosonic atoms in an optical lattice, it neglects terms which may lead to additional features of the phase diagram in certain regimes. Here we comment on a few such possible extensions and their consequences:

Nearest-neighbour interactions: When nearest-neighbour interactions are included in the Bose-Hubbard Hamiltonian, there is evidence for a compressible supersolid phase in two dimensions [90,91]. Such a possibility was first considered by Fisher et al. [75]. This phase is qualified by simultaneous diagonal (crystalline) and off-diagonal (superfluid) long range 
order. However it is worth noting that there is no evidence for this phase in simulations in one dimension $[87,95,97]$.

Disorder: The effects of disorder can be accounted for by a random on-site energy in the Hamiltonian. The disordered model can lead to the prediction of an insulating Boseglass phase which is distinguishable from both the superfluid and Mott insulator phases $[75,86,88,89,96]$. In particular, this phase has a superfluid density of zero (leading to localisation of the bosons) while exhibiting superfluid characteristics such as compressibility and a vanishing energy gap. There is also evidence of an insulating Anderson glass phase for the noninteracting system [86]. Finally, when the effects of on-site disorder are included, it has been shown that the Mott insulator phase is only stable for commensurate occupations less than some critical value [99].

Superlattices: A two-colour superlattice is formed when two optical lattices each with a different lattice spacing (ie. formed by different laser wavelengths) are superimposed. The resulting lattice exhibits a spatially periodic modulation of the lattice depth, and can be described using a cell structure where each cell contains multiple lattice sites. This arrangement introduces an onsite energy shift in the Bose-Hubbard Hamiltonian, and is therefore related to studies of disordered systems. Investigations of ultracold bosonic atoms in two-colour superlattices have revealed that the phase diagram also exhibits a Bose-glass phase $[100,101]$.

\subsection{Overview}

In this part of the thesis we want to introduce approximate methods which should be applicable in both the superfluid and the Mott insulator regimes, and to show their efficacy in the very simplest method used for the Bose-Hubbard model, that of static mean-field theory. We propose a phase space method from quantum optics based on the Q-function representation which involves an approximate reparameterisation of the Bose-Hubbard Hamiltonian in terms of Gaussian variables. The advantage of this methodology lies in the simple description of a wide range of quantum states for the system.

We apply this method to two approximations of the Bose-Hubbard Hamiltonian which we denote as the one site and two site models. A schematic of these models for one dimension is given in Figure 2.1. The one site model treats all intersite correlations (ie. tunneling to adjacent sites) by a mean-field term, which essentially decouples the total Hamiltonian into a sum of one site Hamiltonians. The two site model extends this formulation by explicitly including intersite correlations between two adjacent sites, while treating the interactions with neighbouring sites using the mean-field approximation. The formalism and results for the one and two site models are presented respectively in chapters 3 and 4 , and in section 4.3 we conclude.

For the one site Q-function approach, we find rather good agreement over all regimes, with the advantage that our phase space method can be evaluated very easily - in a matter of seconds on any reasonable workstation. The accuracy is usually about $5 \%$, with the qualitative behavior being 
accurately given. The essence of the result is that the ground state is essentially that wavefunction which minimises the classical energy functional subject to the inequality constraints given by the uncertainty principle in two different forms.

These results are compared with an exact numerical solution to the one site model using arbitrary one site states. In this case, the solution is found by reparameterising the energy in terms of the number variance and finding the maximum mean-field for which the energy is minimised. The formulation is equivalent to the application of the standard Gutzwiller ansatz.

The Q-function formulation is easily extended to the two site model when lattice homogeneity is assumed. The correct description of the two site quantum statistics requires the inclusion of additional constraints which are used to determine the ground state solution. The results here give a good qualitative description of the overall features of the Bose-Hubbard model. However, a shortcoming of the parameterisation on two sites is highlighted by the failure of the method to correctly predict the Mott insulator phase, as determined by a vanishing mean-field, when compared to the results from sections 3.1, 3.2 and 4.2.

The two site model is also solved by exact numerical minimisation with the assumption of lattice symmetry giving a reduced Hilbert space. Here, the ground state results show the pertinent features of the Bose-Hubbard model, with a vanishing mean-field throughout the Mott insulator phase, which is an improvement over the two site Q-function formulation. The results are compared with other reports from the literature, including Density Matrix Renormalisation Group and Quantum Monte-Carlo methods, which yield numerically exact results for one dimensional finite lattices. We then conclude in section 4.3. 


\section{Chapter 3}

\section{One site formulation}

In the preceding chapter, we introduced the Bose-Hubbard model for bosonic atoms interacting on a lattice. Even the simplest form the Bose-Hubbard Hamiltonian (2.1) leads to two vastly different regimes, namely weakly correlated and strongly correlated states. In this chapter, we therefore introduce an approximate method which should be applicable in both of these regimes. This method uses a phase space representation for the one site Hamiltonian (2.6), based on the Q-function, which was introduced in section 1.3. The ground state phase diagram is found by minimising the resulting energy functional subject to quantum mechanical constraints. In section 3.1 we formulate the one site problem in terms of the Q-function, while in section 3.2 we provide the exact numerical solution to same model Hamiltonian for comparison.

\subsection{Q-functions for one site states}

We develop a treatment in terms of the Q-function, following the theory given in Section 4.4.1 of reference [30]. Since the Q-function is always positive and well-defined, and is thus a genuine probability density to which we can apply probabilistic approximations, it can be used to describe a wide range of states for the Bose-Hubbard model which interpolate between the extremes:

i) Weak lattice: in the case that the hopping dominates ( $J$ very large) the ground state is the product of coherent states at each site.

ii) Strong lattice: in the other extreme, when the hopping is negligible, the ground state is the product of eigenstates at each site. If the mean occupation per site is $n$ and $[n]$ denotes the integer part of $n$, then the state at each site is a superposition of number states of the form $\sqrt{\lambda}|[n]\rangle+\sqrt{1-\lambda}|[n]+1\rangle$, where $\lambda$ is chosen to give the correct mean occupation. These states are not Gaussian.

The Gaussian or non-Gaussian nature of the statistics in the Bose-Hubbard model is very important, and one of the virtues of the Q-function is that it is Gaussian when the quantum statistics is also Gaussian [30].

Taking the density operator as the outer product $\rho=|\psi\rangle\langle\psi|$ for a state vector $|\psi\rangle$ and using (1.10), the Q-functions for the three principal kinds of states are: 


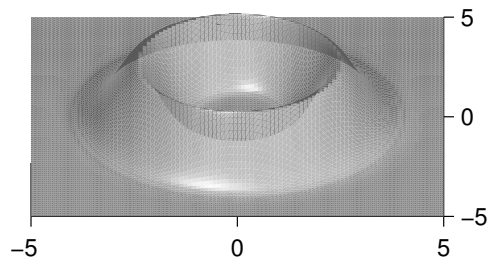

(a) $n=7, \lambda=0$

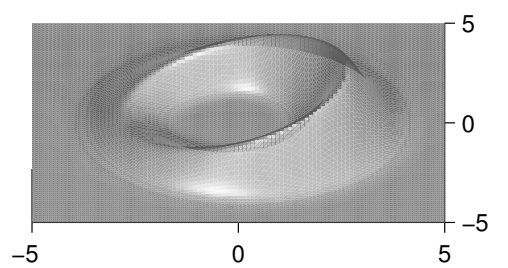

(d) $n=7, \lambda=0.85$

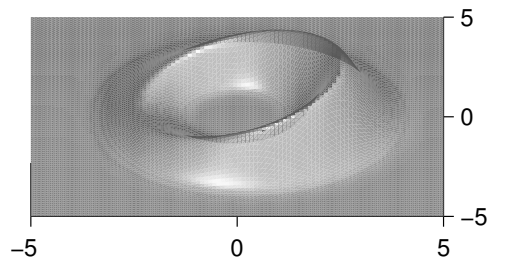

(b) $n=7, \lambda=0.15$

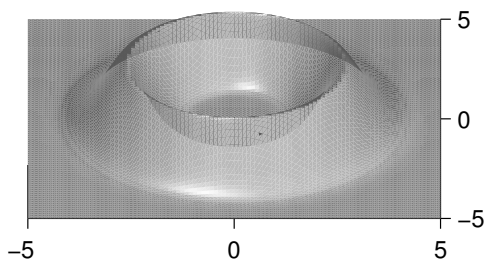

(e) $n=7, \lambda=1$

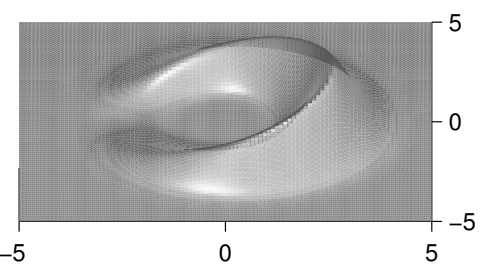

(c) $n=7, \lambda=0.5$

Figure 3.1: Q-function for the superposition of $n=6$ and $n=7$ states in proportion 1:0, $0.85: 0.15,0.5: 0.5,0.15: 0.85,0: 1$; and for a coherent state with $\beta=\sqrt{6}$.

i) Number state: The Q-function for a number state is

$$
Q_{n}\left(\alpha, \alpha^{*}\right)=\frac{1}{\pi} \frac{e^{-|\alpha|^{2}}|\alpha|^{2 n}}{n !} .
$$

Examples of this distribution appear in Figure 3.1 (a) and (e). The distribution has a characteristic ring shape, which is clearly non-Gaussian.

ii) Superposition of number states The Q-function for the superposition of number states with $n-1$ and $n$ atoms in proportions $1-\lambda: \lambda$ is

$$
Q_{\sup \lambda}\left(\alpha, \alpha^{*}\right)=\frac{1}{\pi}\left|\sqrt{1-\lambda}+\alpha \sqrt{\frac{\lambda}{n}}\right|^{2} \frac{e^{-|\alpha|^{2}}|\alpha|^{2(n-1)}}{(n-1) !} .
$$

This is the kind of state expected with an incommensurate filling in the limit of no hopping. The Q-function then varies between a ring shaped distribution for $\lambda=0,1$ to a rather distorted Gaussian distribution at $\lambda=1 / 2$; see Figures 3.1 (a)-(e).

iii) Coherent state In the superfluid case with mean filling per site $n$, where the hopping is dominant, we expect an approximately coherent state with parameter $\beta=\exp (i \theta) \sqrt{n}$, for some real $\theta$, and then the Q-function has the Gaussian form

$$
Q_{\mathrm{coh}, \beta}=\frac{1}{\pi} \exp \left(-|\alpha-\beta|^{2}\right) .
$$

This is plotted in Figure 3.1 (f). 


\subsubsection{Parametrisation of the $Q$-function}

The shape of the probability distributions in Figure 3.1 can be approximately parametrised by writing the random variable $a \rightarrow \alpha$ with

$$
\alpha=(v+\delta) e^{i \theta}
$$

where

a) $v$ is a nonrandom positive real quantity;

b) $\delta$ is a Gaussian random variable with zero mean and variance $\sigma$;

c) $\theta$ is a Gaussian random variable with a mean which is in principle nonzero, but which by choice of phase definition can be chosen to be zero, and with variance $\Delta$. In this case, the averages of powers of $\exp (i \theta)$ are given by

$$
\left\langle e^{p i \theta}\right\rangle=\exp \left(-p^{2} \Delta / 2\right) \equiv c^{p^{2}}
$$

Using properties of Gaussian variables this approximation leads to the values of the antinormal ordered moments:

$$
\begin{aligned}
\langle a\rangle & =v c \\
\left\langle a a^{\dagger}\right\rangle & =\left(v^{2}+\sigma\right) \\
\langle a a\rangle & =\left(v^{2}+\sigma\right) c^{4} \\
\left\langle a a a^{\dagger}\right\rangle & =\left(v^{3}+3 \sigma v\right) c \\
\left\langle a a a^{\dagger} a^{\dagger}\right\rangle & =\left(v^{4}+6 v^{2} \sigma+3 \sigma^{2}\right)
\end{aligned}
$$

Note that the first of these moments (3.6) represents the mean-field. A straightforward extension of this this parameterisation to $L$ sites can be given by the substitution $a_{k} \rightarrow\left(v_{k}+\delta_{k}\right) e^{i \theta_{k}}$ for $k=1,2, \ldots, L$; we make use of this for the two site problem presented in section 4.1.

\section{Validity of parameterisation: values of the parameters in particular cases}

The justification for the above parameterisation is made by comparing the parameterised and exact moments calculated for relevant quantum states. In particular, to fit the kinds of distribution in Figure 3.1 we determine the parameters $v, \sigma$ and $c$ by fitting the moments $\langle a\rangle,\left\langle a a^{\dagger}\right\rangle$ and $\left\langle a a a^{\dagger} a^{\dagger}\right\rangle$. These are given in detail in A.1, where we show that for a wide variety of states we get very tolerable approximations. Thus we can expect a good qualitative description of the system for an arbitrary lattice strength. However, we note that the parameterisation is least accurate in the case of an equal superposition of number states which we will find reflected in the ground state phase diagram in the strong lattice regime. 


\subsubsection{Determination of the ground state}

Using the Hamiltonian (2.6) and the moments (3.6)-(3.10), the average energy in the Q-function representation is

$$
E(n, c, v)=-Z c^{2} v^{2}+u\left(3 n^{2}-2 v^{4}\right) .
$$

To find the ground state solution at a given interaction strength $Z / u$ and mean occupation $n$, this quantity is minimised with respect to the free parameters $v$ and $c$, with the bounds $v \geq 0$ and $0 \leq c \leq 1$ as permitted by the Q-function parameterisation.

\section{Constraints}

The functional (3.11) is a classical distribution that has no local minima so that the minimum must occur on the boundary. However the system should be governed by the quantum mechanical nature of the problem, which we reintroduce in the form of two constraints on the minimisation procedure, one derived from a restriction on the number variance, the other from the uncertainty relation for the conjugate variables of phase and number.

The most significant of the constraints enforced by the quantum mechanical nature of the problem are as follows.

Fractionality constraint If the mean occupation per site is non-integral, the variance of the occupation must be nonzero, and this has a major bearing on the problem, which we shall formulate precisely. We will be considering cases where the mean occupation per site is fixed, so that

$$
\left\langle a a^{\dagger}\right\rangle=n \geq 1
$$

so that

$$
n=v^{2}+\sigma \geq 1 \text {. }
$$

Setting

$$
N=a a^{\dagger}
$$

and using (3.6)-(3.10) the variance of the site occupations is

$$
\begin{aligned}
\operatorname{var}[N] & \equiv\left\langle a a^{\dagger} a a^{\dagger}\right\rangle-\left\langle a a^{\dagger}\right\rangle^{2} \\
& =2 n^{2}-n-2 v^{4}
\end{aligned}
$$

In $n$ is non-integral, with fractional part $\delta n$, then the minimum variance for a given $n$ occurs when only the two occupation numbers which bracket the value $n$ are represented, and then this gives a 
variance of $\delta n(1-\delta n)$, leading to the constraint

$$
\operatorname{var}[N] \geq \delta n(1-\delta n)
$$

and using (3.15) we can write this as

$$
0 \leq v^{4} \leq n^{2}-\frac{1}{2}(n+\delta n(1-\delta n))
$$

Phase-number uncertainty relations The uncertainty principle enters when we consider that phase and number are conjugate variables. We can write a rigorous uncertainty relationship using

$$
X=\frac{a+a^{\dagger}}{2} ; \quad Y=\frac{a-a^{\dagger}}{2 i}
$$

so that we have the commutation relations

$$
[N, X]=-i Y ; \quad[N, Y]=i X
$$

from which follow an uncertainty relation

$$
\left\langle\delta Y^{2}\right\rangle \operatorname{var}[N] \geq \frac{1}{4}\langle X\rangle^{2}
$$

In our formulation we have chosen a zero mean phase so that $\langle Y\rangle=0$ (see (A.13)) and thus $\left\langle\delta Y^{2}\right\rangle=\left\langle Y^{2}\right\rangle$. Using (A.13-A.17), and antinormally ordering all the products involved, we find

$$
\left\langle\delta Y^{2}\right\rangle=\frac{n}{2}\left(1-c^{4}\right)-\frac{1}{4}
$$

and thus

$$
\left[\frac{n}{2}\left(1-c^{4}\right)-\frac{1}{4}\right]\left(2 n^{2}-n-2 v^{4}\right) \geq \frac{1}{4} v^{2} c^{2} .
$$

\section{Results}

Minimising (3.11) with the constraints (3.17) and (3.22) leads to the ground state phase diagram shown in Figure 3.3. These results compare well with the exact solution shown in Figure 2.2, particularly with confirmation of the Mott insulator phase at commensurate occupations for a strong lattice (small $Z / u$ ), and of the superfluid phase for a weak lattice (large $Z / u$ ).

The results are dominated by the interplay between the constraints, which is made evident by considering Figure 3.2. In the strong lattice regime, the ground state solution is given at the intersection of the fractionality constraint (3.17) which determines the upper bound for $v$, and the phase-number uncertainty constraint (3.22), shown by the solid curve. Specifically for $Z / u=0.5$, and for an integer $n=1$, this occurs at $c=0$ and the resulting vanishing mean-field is indicative of the Mott insulator phase; for a non-integer $n=1.5$ the solution occurs for $c \neq 0$ so that there is a non-zero mean-field corresponding to a superfluid component. Conversely for a weak lattice, 


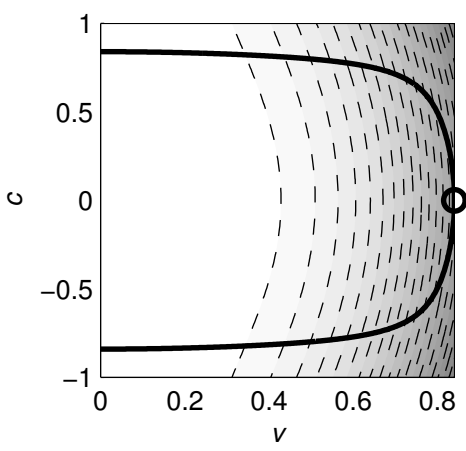

(a) $n=1, Z / u=0.5$

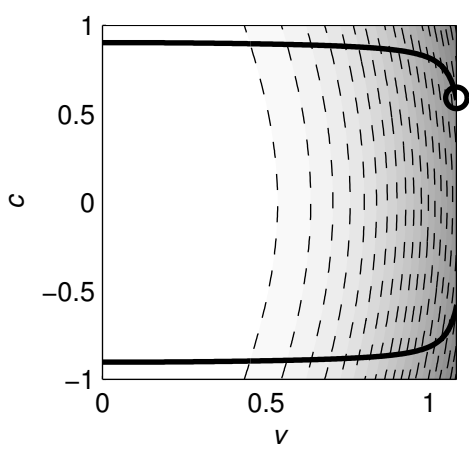

(b) $n=1.5, Z / u=0.5$

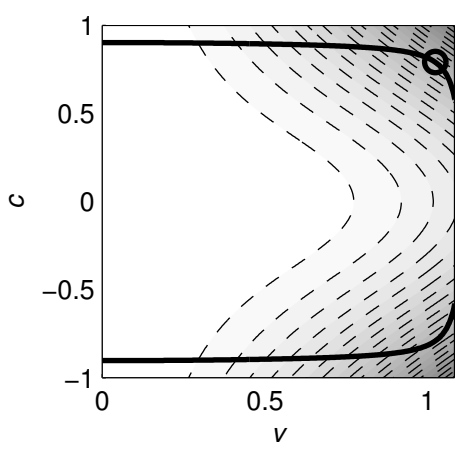

(c) $n=1.5, Z / u=10$

Figure 3.2: Level curves of energy (3.11) in the Q-function representation for three different cases are indicated by the dashed curves, with the energy decreasing for darker regions; the permissible parameter space for $v$ and $c$ is given by the bounds $v \geq 0$ and $0 \leq|c| \leq 1$ (for purely illustrative purposes we allow $c$ to be negative here because the problem is symmetrical about $c=0$ ) where $v$ is further bounded by (3.17). Since (3.11) has no local minima, the minimum energy occurs within the shown bounded region and subject to the constraint (3.22) derived from the phase-number uncertainty relation which is shown by the solid curve. The solution in each case is indicated by a circle. In the first case $(n=1, Z / u=0.5)$ this occurs for $c=0$ so that the mean-field is zero; in the second case $(n=1.5, Z / u=0.5)$ this occurs for a non-zero $c$ so that there is a mean-field. In both these cases, the solution saturates the upper bound for $v$. Conversely, in the third case $(n=1.5, Z / u=10)$ where the lattice is weak, the solution occurs within the bounded region.

with $n=1.5$ and $Z / u=10$, the solution is determined solely by (3.22).

The fact that the quantum mechanical constraints dominate the problem is not unexpected as the phase transition between the Mott insulator and superfluid is driven by quantum fluctuations.

We note that for the Q-function results at half-integer values of $n$, the mean-field remains constant over some range of $Z / u$ in the strong lattice regime. This contrasts with the numerically exact results obtained by a self-consistent mean-field approach as shown in Figure 2.2 where the mean-field is a monotonically increasing function of $Z / u$. This difference arises from the weaker approximation of the Q-function parameterisation in the case of an equal superposition of number states as shown in Figure 3.1 (c), and quantified in Appendix A.1.

\subsection{Exact numerical minimisation of one site problem}

The inequalities (3.22) and (3.17) restrict the mean-field to a certain maximum value, but this maximum may not be optimal. Let us then pose the question: What is the largest value of the mean-field for a given mean number and variance. This value determines the minimum energy and permits the following basic strategy for finding the ground state phase diagram. Firstly, reparameterise the mean-field in terms of the mean number occupation and number variance; and secondly, minimise the energy with respect to the number variance at a fixed relative interaction strength $z t / u$ and mean occupation. The problem is then essentially reduced to a minimisation of energy with respect to a single variable - the number variance. 

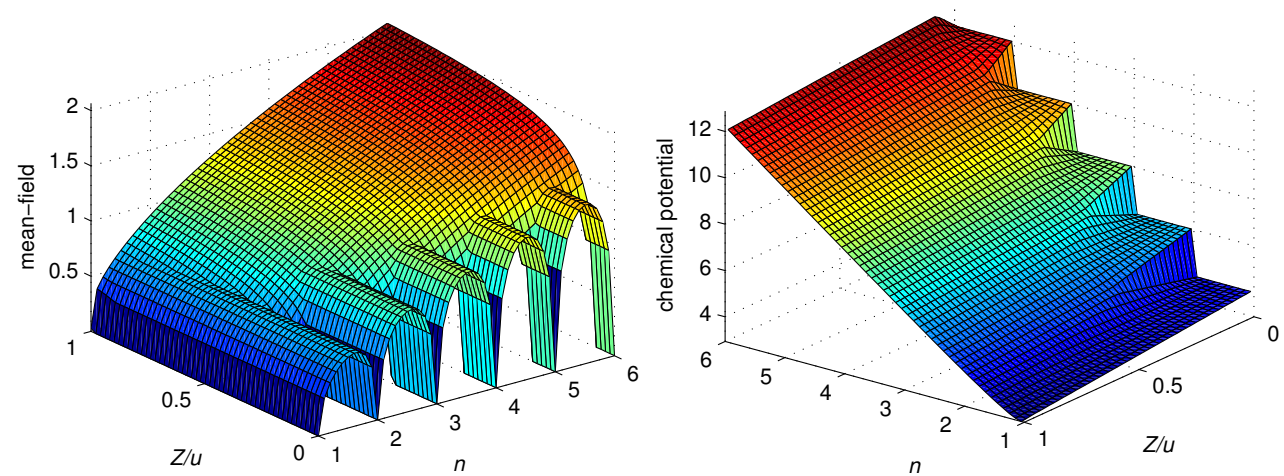

Figure 3.3: Ground state results for Bose-Hubbard model with Q-function representation; the mean-field and chemical potential are shown as a function of relative interaction strength $Z / u$ and (anti-normal ordered) occupation $n$.

\subsubsection{Formulation using one site states}

Here we give an exact numerical procedure for determining the ground state of the Bose-Hubbard model in the one site mean-field approximation. A one site quantum state can be written as a superposition of number states

$$
|s\rangle=\sum_{n} c_{n}|n\rangle
$$

where the usual normalisation condition $(1=\langle s \mid s\rangle)$ applies. In this basis the mean-field is

$$
\langle X\rangle=\left\langle s\left|\frac{a+a^{\dagger}}{2}\right| s\right\rangle=\Re\left\{\sum \sqrt{n} c_{n-1}^{*} c_{n}\right\}
$$

The Hamiltonian (2.6) leads to the expression for the one site energy in the mean-field approximation

$$
\langle E\rangle=-Z\langle X\rangle^{2}+u\left(2+\operatorname{var}[N]+\bar{n}^{2}+3 \bar{n}\right)
$$

where $\bar{n} \equiv\left\langle a^{\dagger} a\right\rangle$ is the (normal ordered) mean number and $\operatorname{var}[N]=\overline{n^{2}}-\bar{n}^{2}$ is the number variance of atoms on each site (note the normal and anti-normal ordered means are related by $\left.\left\langle a^{\dagger} a\right\rangle=\left\langle a a^{\dagger}\right\rangle-1\right)$. Since the chemical potential term has been omitted in (2.2), we treat the system using the canonical ensemble by taking a fixed number of atoms in the lattice, that is by explicitly using the constraint of fixed mean occupation.

Clearly, the energy functional (3.25) is then minimised when the mean-field $\langle X\rangle$ is a maximum and subject to the constraints of normalisation and fixed number mean and variance. In terms of the one site state (3.23) these constraints are given by

$$
\begin{aligned}
& \phi_{1}=\sum_{n}\left|c_{n}\right|^{2}-1=0 \text { (normalisation) } \\
& \phi_{2}=\sum_{n}\left|c_{n}\right|^{2} n-\bar{n}=0 \text { (fixed mean) }
\end{aligned}
$$




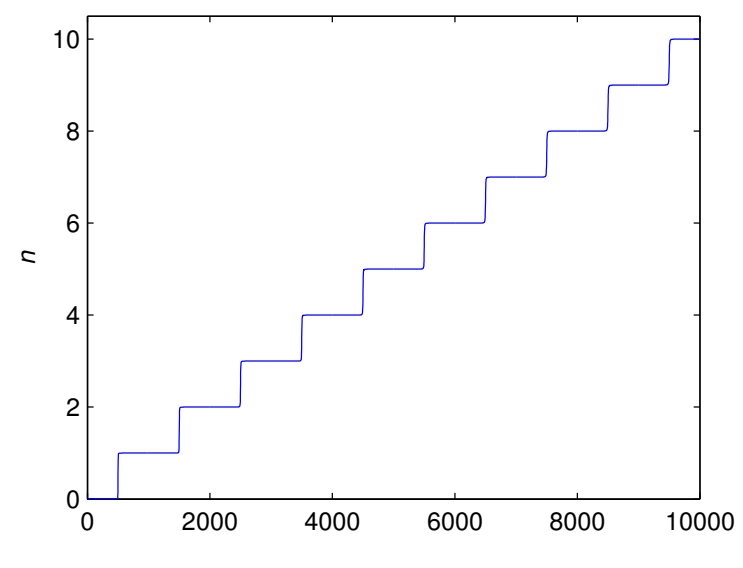

Figure 3.4: Mean number occupation $n$ as a function of $\mu$ at $\nu=-500$ for the one site model. The Lagrange multiplier $\mu$ plays the role of (but is not equal to) the chemical potential; the horizontal regions are indicative of the system near the incompressible Mott insulator phase where $\partial \bar{n} / \partial \mu=$ 0 .

$$
\phi_{3}=\sum_{n}\left|c_{n}\right|^{2} n^{2}-\overline{n^{2}}=0 \text { (fixed variance) }
$$

Moreover, since the constraints are independent of phase, the mean-field (3.24) is maximised by choosing the set of $c_{n}$ to be real and positive at fixed $\left|c_{n}\right|$. To deal with the problem of constrained optimisation we apply the method of Lagrange multipliers; the mean-field is maximised when

$$
0=\frac{\partial\langle X\rangle}{\partial c_{n}}-\frac{1}{2} \lambda \frac{\partial \phi_{1}}{\partial c_{n}}+\frac{1}{2} \mu \frac{\partial \phi_{2}}{\partial c_{n}}+\frac{1}{2} \nu \frac{\partial \phi_{3}}{\partial c_{n}}
$$

Note the scalar factors that appear before the Lagrange multipliers $(\lambda, \mu$ and $\nu)$ are included for convenience. Equation (3.29) along with equations (3.24) and (3.26)-(3.28) then lead to

$$
\sqrt{n} c_{n-1}+\sqrt{n+1} c_{n+1}+\left(\mu n+\nu n^{2}\right) c_{n}=\lambda c_{n}
$$

This is a Hermitian eigenvalue problem which has only one eigenvector solution with the set $c_{n}$ all positive, since the solutions form an orthonormal basis (solutions with some $c_{n}$ vanishing may cause ambiguities in principle however).

\subsubsection{Numerical Solutions}

Numerical solutions of (3.30) determine the maximum mean-field in terms of $\operatorname{var}[N]$ and $\bar{n}$. By choosing a fixed $Z / u$ and $\bar{n}$ the calculation of the ground state energy then amounts to a direct minimisation of (3.25) with respect to the variance $\operatorname{var}[N]$.

We outline the procedure as follows. First, for an appropriate range of the Lagrange multipliers $(\mu, \nu)$ the eigenvector solutions of (3.30) are used to calculate corresponding values of $\bar{n}, \operatorname{var}[N]$ and the maximum mean-field $\langle X\rangle$. A fixed value of $\nu$ determines a $\bar{n}(\mu)$ curve (see for example Fig.3.4) which is inverted using linear interpolation - with an additional optimisation step - to give $\mu$ on a uniform set of $\bar{n}$ points. That is, each value of $\bar{n}$ determines a curve in $\mu$ and $\nu$ from which 
the dependence of $\langle X\rangle$ on $\operatorname{var}[N]$ is determined from solutions to (3.30). Since this relationship is determined on a finite set of points, it is necessary in practice to use cubic interpolation to determine $\langle X\rangle$ for arbitrary $\operatorname{var}[N]$.

Calculations of $\langle X\rangle$ as a function of $\operatorname{var}[N]$ are shown in Fig.3.5 for commensurate and incommensurate mean site occupations; the corresponding curves which arise from the uncertainty relation (3.22) are shown to be very similar. Note that for commensurate mean occupations the mean-field and variance simultaneously approach zero, corresponding to the Mott insulator phase (a pure number state). In contrast, in the incommensurate case neither the mean-field nor variance approach zero; even in the strong lattice regime there is a non-zero superfluid component, corresponding to a superposition of two number states. Using these curves, the energy (3.25) can be minimised with respect to $\operatorname{var}[N]$. This leads to ground state results that are the same numerically as those of the self-consistent mean-field approach shown in Fig.2.2.

Although the difference in the two sets of curves shown in Fig.3.5 appears slight, it is entirely responsible for the notable difference between the exact one site calculation (Fig.2.2) and the approximate phase-space calculation (Fig.3.3). In the incommensurate case, the fact that the exact curves drop rapidly as $\operatorname{var}[N] \rightarrow 1 / 4$ is responsible for the sloping behaviour of the mean-field arches shown in Fig.2.2 as opposed to the level behaviour shown in Fig.3.3.
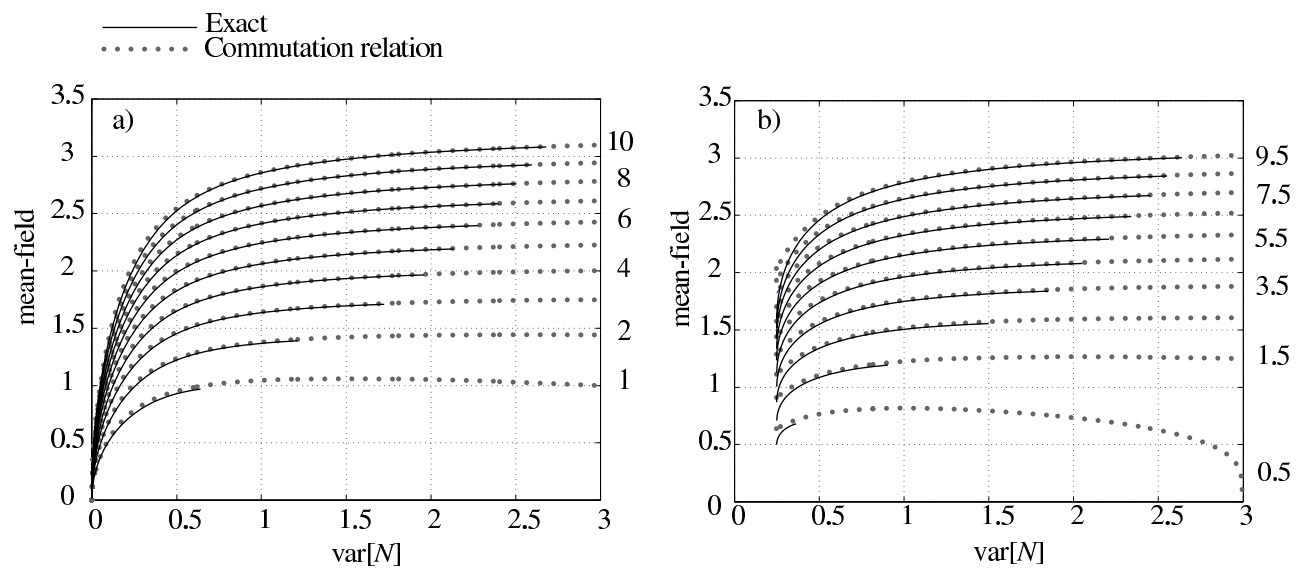

Figure 3.5: Mean-field versus variance for commensurate (a) and incommensurate (b) mean site occupations for the one site case. The mean occupation number is indicated on the right axis of each plot.

Numerical calculations were performed using the MATLAB software package. The state space was truncated with $n \leq 40$ as this provided a good trade-off between computational efficiency and accuracy (ie. this truncation introduced negligible error to solutions in the region of interest $\bar{n} \leq 10)$. Note, to cover a sufficient $\bar{n}$ and $\operatorname{var}[N]$ range, $(\mu, \nu)$ were chosen from the triangular region with $-800 \leq \nu \leq-0.01$ and $0 \leq \mu \leq-40 \nu$. The minimisation procedure used the MATLAB fminbnd (bounded minimisation) function, with a lower bound for the number variance determined by the fractionality constraint (3.16). 


\subsubsection{Calculating the phase transition boundary}

To check the validity of the one site formulation near the transition point, it is useful to calculate the position of the phase boundary between the superfluid and Mott insulator phases. This can be done approximately by a perturbation method where the ground state energy is determined by states near the transition point. We consider a normalised state of commensurate occupation $\bar{n}$ near the transition point

$$
|s\rangle=\sqrt{1-2 \lambda}|\bar{n}\rangle+\sqrt{\lambda}\left(e^{i \theta_{1}}|\bar{n}-1\rangle+e^{i \theta_{2}}|\bar{n}+1\rangle\right)
$$

where $\lambda$ is a real and small; $\lambda=0$ corresponds to the Mott insulating phase with a commensurate occupation $\bar{n}$. States with $\lambda \geq 0$ characterise the superfluid phase where the mean-field is nonzero. The corresponding one site energy (3.25) is given by

$$
\begin{aligned}
\bar{E}= & -\lambda(1-2 \lambda)\left(\sqrt{\bar{n}} \cos \theta_{1}+\sqrt{\bar{n}+1} \cos \theta_{2}\right)^{2} \\
& +\bar{u}\left(2+2 \lambda+\bar{n}^{2}+3 \bar{n}\right)
\end{aligned}
$$

Note that in scaling by the factor $1 / z t$ this form of the energy is dimensionless and we have defined the ratio $\bar{u} \equiv u / z t$ for the relative interaction strength. The ground state is determined by the minimum energy with respect to variations in the free parameters: $\lambda, \theta_{1}$ and $\theta_{2}$. Clearly this occurs for $\theta_{1}=\theta_{2}=0$ (the result $\theta_{1}=\theta_{2}=\pi$ gives the same ground state energy and is equivalent to a change in the sign of the mean-field) and when

$$
\frac{\partial \bar{E}}{\partial \lambda}=0=-(1-4 \lambda)\left(\sqrt{\bar{n}} \cos \theta_{1}+\sqrt{\bar{n}+1} \cos \theta_{2}\right)^{2}+2 \bar{u}
$$

The transition to a Mott insulator phase occurs in the limit $\lambda \rightarrow 0$ when the mean-field goes to zero. Applying this condition, the critical point for the transition is then given by

$$
\bar{u}_{c}=\frac{1}{2}(\sqrt{\bar{n}}+\sqrt{\bar{n}+1})^{2}
$$

For values of the relative interaction strength above the critical value $\bar{u}>\bar{u}_{c}$ the ground state solution saturates the bound at $\lambda=0$ and the system remains in the Mott insulator phase.

\section{Comparison to other work}

In order to compare our results with those reported elsewhere we define the parameter

$$
\bar{U}_{c} \equiv 2(u / z t)_{c}
$$

to describe the relative interaction strength at the critical point. The factor of 2 is included because the on-site interaction term $u$ in the Hamiltonian (2.2) is equivalent to the term $U / 2$ which has been used elsewhere (see [68] and [66] for example).

Equation (3.34) then becomes $\bar{U}_{c}=(\sqrt{\bar{n}}+\sqrt{\bar{n}+1})^{2}$, an expression also found elsewhere $[68,81]$; this yields the following values for the transition: $\bar{U}_{c}=5.83$ for $\bar{n}=1, \bar{U}_{c}=9.90$ for 
$\bar{n}=2, \bar{U}_{c}=13.93$ for $\bar{n}=3$. 


\section{Chapter 4}

\section{Two site formulation}

The one site formulation, presented in the preceding chapter, necessarily neglects correlations between neighbouring sites in the mean-field approximation. In this chapter, we improve on this situation by using a Hamiltonian that explicitly includes hopping between two adjacent sites while still treating interactions with other neighbouring sites with the mean-field approximation. In section 4.1 we formulate the two site problem in terms of the Q-function, while in section 4.2 we provide the exact numerical solution to same model Hamiltonian for comparison, as well as comparing the one site and two site models, before concluding in section 4.3.

\subsection{Q-function parameterisation on two sites}

We formulate the two site problem as follows: consider two adjacent sites labeled by 1 and 2 corresponding to sites $i$ and $i+1$ respectively as shown in Fig. 2.1(b). Similarly to the one site case (2.2) we can write the Bose-Hubbard Hamiltonian in a two site mean-field approximation as

$$
\begin{aligned}
H_{\text {two site }}= & -t\left[a_{1} a_{2}^{\dagger}+a_{2} a_{1}^{\dagger}+(2 d-1)\left(\frac{1}{2} \mathcal{E}\left(a_{1}+a_{1}^{\dagger}\right)+\frac{1}{2} \mathcal{E}\left(a_{2}+a_{2}^{\dagger}\right)\right)\right] \\
& +u\left(a_{1} a_{1} a_{1}^{\dagger} a_{1}^{\dagger}+a_{2} a_{2} a_{2}^{\dagger} a_{2}^{\dagger}\right)
\end{aligned}
$$

for a homogeneous lattice of dimension $d$ where $\mathcal{E}=\left\langle a_{k}\right\rangle$ (for $k \neq 1,2$ ) is the mean-field representing the interaction with nearest neighbours, which as with the one site model, by a choice of phase is taken as real. Here $t$ is the tunneling amplitude. (Note the change of notation from chapters 2 and 3 where the tunneling amplitude was denoted by $J$.)

In this case the Q-function parameterisation becomes

$$
\begin{aligned}
& a_{1} \rightarrow \alpha_{1}=\left(v_{1}+\delta_{1}\right) e^{i \theta_{1}} \\
& a_{2} \rightarrow \alpha_{2}=\left(v_{2}+\delta_{2}\right) e^{i \theta_{2}}
\end{aligned}
$$

We define the intersite correlation operators

$$
b \equiv a_{1} a_{2}^{\dagger} ; \quad b^{\dagger} \equiv a_{2} a_{1}^{\dagger}
$$


The corresponding moments are

$$
\langle b\rangle=\left\langle b^{\dagger}\right\rangle=\left(v_{1} v_{2}+w\right) g
$$

where we have set

$$
\begin{gathered}
w \equiv\left\langle\delta_{1} \delta_{2}\right\rangle \\
g \equiv\left\langle e^{i\left(\theta_{1}-\theta_{2}\right)}\right\rangle
\end{gathered}
$$

and assumed that the phase and number correlations are independent; although this assumption is possibly not strictly valid, without it the formalism becomes significantly more complicated.

The two site Hamiltonian (4.1) leads to the expression for the average energy

$$
\begin{aligned}
E= & \left\langle H_{\text {two site }}\right\rangle \\
= & -\bar{t}\left[2\left(v_{1} v_{2}+w\right) g+(2 d-1)\left(v_{1}^{2} c_{1}^{2}+v_{2}^{2} c_{2}^{2}\right)\right] \\
& +3 n_{1}^{2}-2 v_{1}^{4}+3 n_{2}^{2}-2 v_{2}^{4}
\end{aligned}
$$

which has been normalised by dividing through by $u$ and defining the relative interaction strength $\bar{t} \equiv t / u$.

\subsubsection{Constraints}

Following the one site case, to find the ground state solution, the energy (4.8) is minimised subject to constraints arising from any applicable uncertainty relations. In particular, in addition to the constraints (3.17) and (3.22) that have already been introduced for the one site statistics, we can derive further constraints that account for the two site statistics by considering commutation relations between bilinear operators.

\section{Variances and covariance}

In deriving the necessary constraints for the two site problem, we first consider the following operators

$$
\begin{aligned}
& N \equiv \frac{N_{1}+N_{2}}{2} \\
& M \equiv \frac{N_{1}-N_{2}}{2}
\end{aligned}
$$

The corresponding variances for each of these operators are given by

$$
\begin{aligned}
& \operatorname{var}[N]=1 / 4\left(\operatorname{var}\left[N_{1}\right]+\operatorname{var}\left[N_{2}\right]+2 \operatorname{cov}\left(N_{1}, N_{2}\right)\right) \\
& \operatorname{var}[M]=1 / 4\left(\operatorname{var}\left[N_{1}\right]+\operatorname{var}\left[N_{2}\right]-2 \operatorname{cov}\left(N_{1}, N_{2}\right)\right)
\end{aligned}
$$


where the number covariance function is given by

$$
\begin{aligned}
\operatorname{cov}\left(N_{1}, N_{2}\right) & \equiv\left\langle N_{1} N_{2}\right\rangle-\left\langle N_{1}\right\rangle\left\langle N_{2}\right\rangle \\
& =2\left(2 v_{1} v_{2} w+w^{2}\right)
\end{aligned}
$$

with the one site variances, $\operatorname{var}\left[N_{1}\right]$ and $\operatorname{var}\left[N_{2}\right]$, given analogously to (3.15).

\section{Commutation relations}

We define the following operators

$$
\begin{gathered}
V \equiv \frac{b+b^{\dagger}}{2} \\
W \equiv \frac{b-b^{\dagger}}{2 i}
\end{gathered}
$$

$\langle V\rangle$ is the intersite correlation and gives a measure of particle exchange, or tunneling, between adjacent sites. Eqs. (4.14) and (4.15) have the following commutation relations

$$
\begin{aligned}
& {[V, M]=i W} \\
& {[M, W]=i V} \\
& {[W, V]=i M}
\end{aligned}
$$

\section{Quantum mechanical constraints}

Using (4.16)-(4.18) and noting that $\langle W\rangle=0$, we can write the corresponding uncertainty relations

$$
\begin{gathered}
\left(\left\langle V^{2}\right\rangle-\langle V\rangle^{2}\right) \operatorname{var}[M] \geq \frac{1}{4}\left\langle[\delta V, \delta M]_{+}\right\rangle^{2} \\
\operatorname{var}[M]\left\langle W^{2}\right\rangle \geq \frac{1}{4}\langle V\rangle^{2}+\frac{1}{4}\left\langle[\delta M, \delta W]_{+}\right\rangle^{2} \\
\left(\left\langle V^{2}\right\rangle-\langle V\rangle^{2}\right)\left\langle W^{2}\right\rangle \geq \frac{1}{4}\langle M\rangle^{2}+\frac{1}{4}\left\langle[\delta V, \delta W]_{+}\right\rangle^{2}
\end{gathered}
$$

with

$$
\begin{gathered}
\left\langle V^{2}\right\rangle=\frac{1+g^{4}}{2}\left(n_{1} n_{2}+\operatorname{cov}\left(N_{1}, N_{2}\right)\right)-\frac{n_{1}+n_{2}}{4} \\
\langle V\rangle=\left(v_{1} v_{2}+w\right) g \\
\left\langle W^{2}\right\rangle=\frac{1-g^{4}}{2}\left(n_{1} n_{2}+\operatorname{cov}\left(N_{1}, N_{2}\right)\right)-\frac{n_{1}+n_{2}}{4}
\end{gathered}
$$

\section{Applying homogeneity}

These constraints further simplify when homogeneity is assumed. In particular, if we assume the ground state is symmetric to the $1 \leftrightarrow 2$ interchange, we can set

$$
v_{1}=v_{2}=v
$$




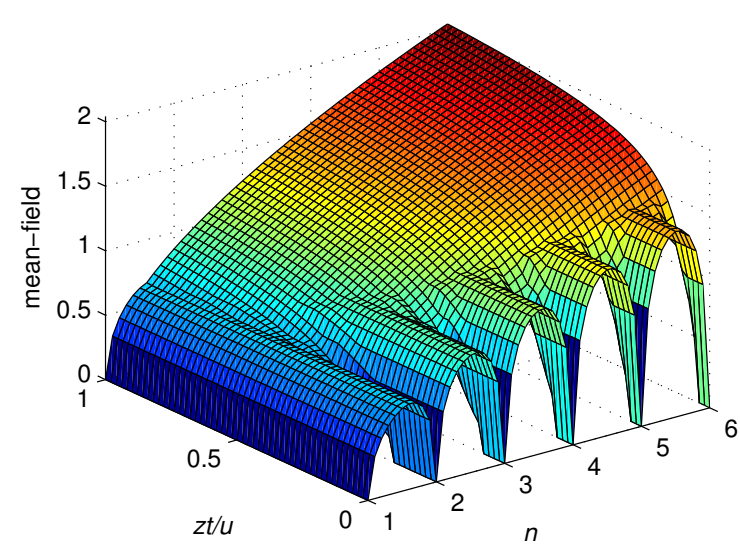

(a) Mean-field, $d=1$

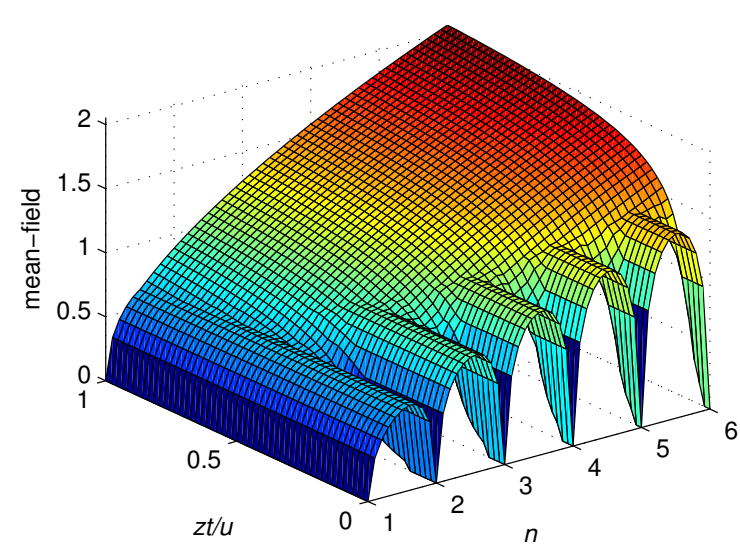

(c) Mean-field, $d=2$

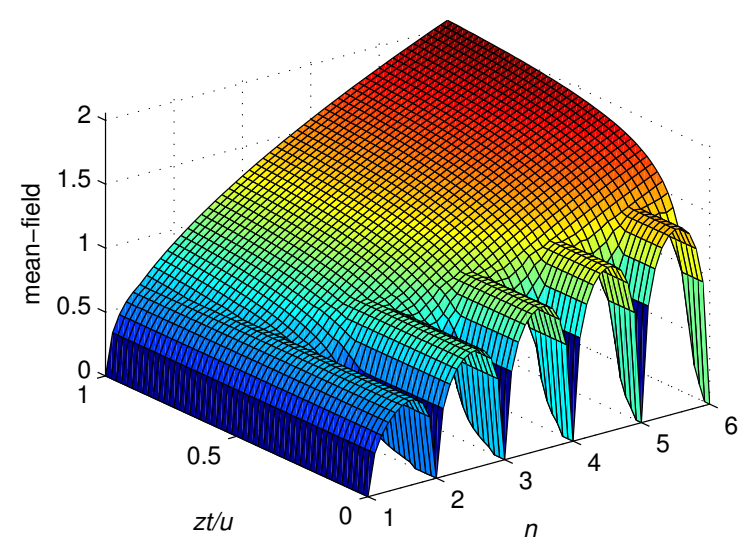

(e) Mean-field, $d=3$

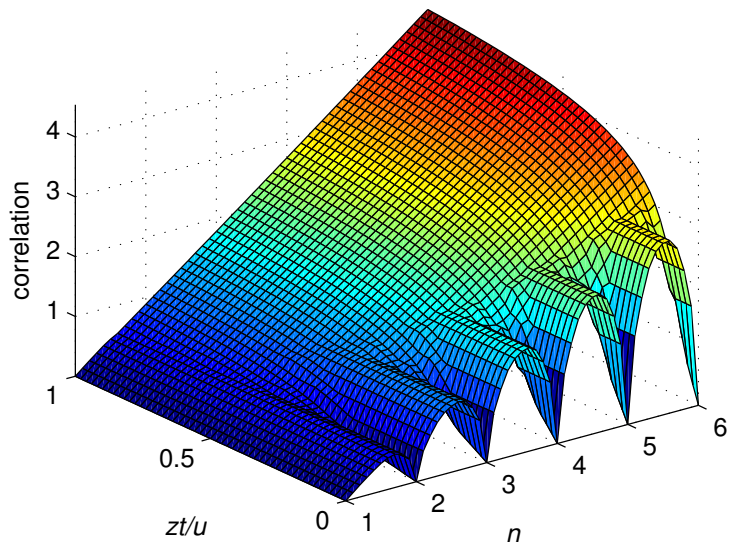

(b) Intersite correlation, $d=1$

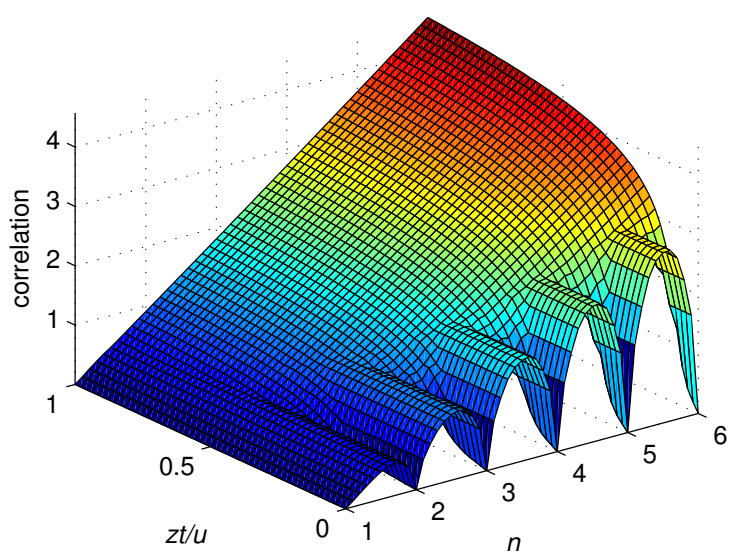

(d) Intersite Correlation, $\mathrm{d}=2$

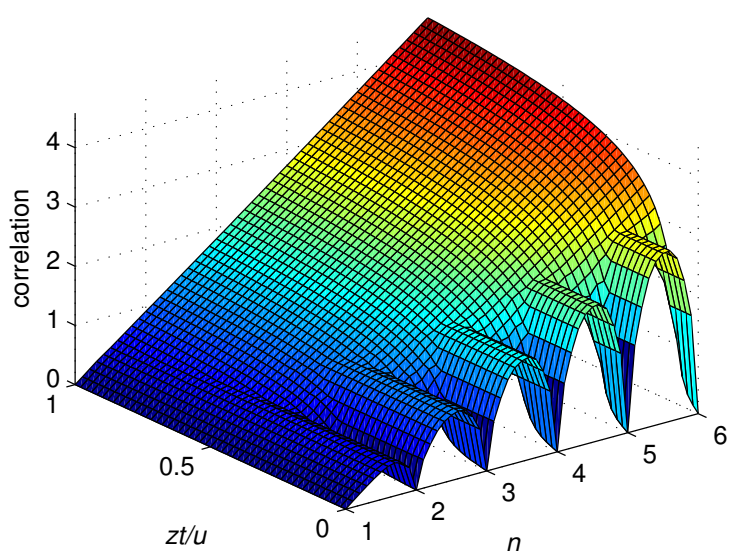

(f) Intersite Correlation, $\mathrm{d}=3$

Figure 4.1: Ground state phase diagram for two site Bose-Hubbard model using the Q-function representation in the mean-field approximation. The mean-field and intersite correlations are shown as a function of the mean (anti-normal ordered) occupation $n$ and the relative interaction strength $z t / u$. 


$$
\begin{gathered}
c_{1}=c_{2}=c \\
n_{1}=n_{2}=n
\end{gathered}
$$

Clearly then

$$
\langle M\rangle=0
$$

Moreover, under the above interchange the following moments are equal

$$
\begin{aligned}
& \left\langle b^{\dagger} N_{2}\right\rangle=\left\langle b N_{1}\right\rangle \\
& \left\langle b N_{2}\right\rangle=\left\langle b^{\dagger} N_{1}\right\rangle
\end{aligned}
$$

It follows that the anti-commutators in equations (4.19)-(4.21) are given by

$$
\begin{aligned}
& {[\delta V, \delta M]_{+}=0} \\
& {[\delta M, \delta W]_{+}=0} \\
& {[\delta V, \delta W]_{+}=0}
\end{aligned}
$$

\section{Two site phase-number uncertainty relation}

Similarly, the commutation relation $(i=1,2)$

$$
\left[N, Y_{i}\right]=\frac{1}{2} i X_{i}
$$

leads to the constraint

$$
\operatorname{var}[N]\left\langle Y_{i}^{2}\right\rangle \geq \frac{1}{16}\left\langle X_{i}\right\rangle^{2}
$$

\section{Two site fractionality constraint}

A lower limit on the two site variance $\operatorname{var}[N]$ can also be given by considering two site states with minimum variance. Such a state has the form

$$
\begin{aligned}
|s\rangle= & \sqrt{\alpha}|[n],[n]\rangle+\sqrt{\beta / 2}(|[n],[n]+1\rangle+|[n]+1,[n]\rangle) \\
& +\sqrt{\gamma}|[n]+1,[n]+1\rangle
\end{aligned}
$$

where $[n]=n-\delta n$ is the integer part of $n, \delta n$ being the fractional part. We then have

$$
\begin{aligned}
& \operatorname{var}\left[N_{i}\right]=\frac{\beta}{2}\left(1-\frac{\beta}{2}\right) \\
& \operatorname{cov}\left(N_{1}, N_{2}\right)=-\beta^{2} / 4
\end{aligned}
$$

so that we can write the minimum variance

$$
\operatorname{var}[N]_{\text {min }}=\frac{1}{4} \beta(1-\beta)
$$


Using (4.11) and applying homogeneity this leads to the fractionality constraint for the two site variance

$$
n^{2}-\frac{1}{2} n-v^{4}+2 v^{2} w+w^{2} \geq \frac{1}{4} \beta(1-\beta)
$$

We note the following two cases

1. $0 \leq \delta n \leq 0.5$ : In this case $\gamma=0$ and $\beta=2 \delta n$.

2. $0.5 \leq \delta n \leq 1:$ In this case $\alpha=0$ and $\beta=2(1-\delta n)$.

\subsubsection{The ground state solution}

To find the ground state solution we assume homogeneity and minimise the two site energy (4.8) at a given occupation $n$ and relative interaction strength $\bar{t}$ with respect to the free parameters $v$, $c, w$ and $g$. This is done subject to the constraints (3.17), (3.22), (4.19)-(4.21), (4.35) and (4.40). The parametrisation requires that

$$
v \geq 0
$$

Additionally, by noting the variables $c$ and $g$ both take the form $\left\langle e^{i \phi}\right\rangle$, the following bounds apply

$$
\begin{aligned}
& 0 \leq c \leq 1 \\
& 0 \leq g \leq 1
\end{aligned}
$$

\subsubsection{Results}

The results of this calculation are shown in Fig.4.1. The solutions reproduce the general features of the Bose-Hubbard model in the superfluid limit and for incommensurate occupations in the limit of zero tunneling. However, in the case of a commensurate mean occupation, the Q-function parametrisation on two sites does not correctly demonstrate the existence of the Mott insulator phase. Specifically we find that for a three dimensional lattice, the mean-field only vanishes in the limit of $z t / u \rightarrow 0$. Moreover, in the one and two dimensional cases, the predicted Mott insulator phase only occurs for very small values of $z t / u$. See sections 3.1, 3.2 and 4.2 for comparison; in particular, the results from section 4.2 show a well defined Mott phase with a vanishing mean-field. Improved agreement can be seen between the intersite correlations calculated by the Q-function representation and the exact numerical results of section 4.2. In both cases the intersite correlations vanish in the limit of zero tunneling for commensurate occupations.

\subsection{Exact numerical minimisation of two site problem}

Following the one site formulation discussed in section 3.2.1, we apply a two site generalisation of the exact numerical calculation using arbitrary two site states. The formulation uses the assumption that the lattice is translationally invariant so that we can apply symmetry for the two sites in the ground state. 
The Hamiltonian (4.1) leads to an expression for the two site energy

$$
\begin{aligned}
\langle E\rangle_{\text {two site }}= & -2 t\left[\langle V\rangle+(2 d-1)\langle X\rangle^{2}\right] \\
& +2 u\left(2+\operatorname{var}[N]+\bar{n}^{2}+3 \bar{n}\right)
\end{aligned}
$$

where $V$ is the intersite correlation operator given by (4.14) and

$$
\hat{X}=\frac{1}{2}\left(a_{i}+a_{i}^{\dagger}\right)
$$

is the mean-field operator, which by homogeneity, does not depend on the site index.

Note that in the limit that $\langle V\rangle \rightarrow\langle X\rangle^{2}$ the two site energy tends to twice the one site energy. This corresponds to the case where the two site state is factorisable $\left|\psi_{12}\right\rangle \rightarrow\left|\psi_{1}\right\rangle\left|\psi_{2}\right\rangle$ and the one site behaviour is returned.

In general, for $n$ and $m$ atoms on sites 1 and 2 respectively, the two site quantum state can be written as

$$
\begin{aligned}
|s\rangle= & \sum_{n, m} c_{n, m}|n, m\rangle \\
= & \sum_{N, p \neq 0} C_{N, p}\left(\left|\frac{1}{2}(N+p), \frac{1}{2}(N-p)\right\rangle+\left|\frac{1}{2}(N-p), \frac{1}{2}(N+p)\right\rangle\right) \\
& +\sum_{N, p=0} C_{N, 0}\left|\frac{1}{2} N, \frac{1}{2} N\right\rangle
\end{aligned}
$$

where we have introduced the quantum numbers $N=n+m$ and $p=|n-m| \leq N$ to account for the lattice symmetry $c_{n, m}=c_{m, n}$ (we should note this symmetry argument can only be applied when the ground state exhibits homogeneity). The correlation function is

$$
\begin{aligned}
\langle V\rangle= & \left\langle a_{i} a_{j}^{\dagger}\right\rangle \\
= & \sum_{n, m} c_{n, m} c_{n+1, m-1} \sqrt{m(n+1)} \\
= & \sum_{N, p \geq 0} C_{N, p} C_{N, p+2} \sqrt{(N-p)(N+p+2)} \\
& +\frac{1}{2} \delta_{p, 1} \sum_{N}\left(C_{N, 1}\right)^{2}(N+1)
\end{aligned}
$$

and the mean-field is

$$
\begin{aligned}
\langle X\rangle= & \left\langle a_{i}\right\rangle \\
= & \sum_{n, m} c_{n, m} c_{n+1, m} \sqrt{n+1} \\
= & \frac{1}{\sqrt{2}} \sum_{N, p>0} C_{N, p}\left[C_{N+1, p+1} \sqrt{N+p+2}+C_{N+1, p-1} \sqrt{N-p+2}\right] \\
& +\frac{1}{\sqrt{2}} \delta_{p, 0} \sum_{N} C_{N, 0} C_{N+1,1} \sqrt{N+2}
\end{aligned}
$$


Since the second term in (4.44) is always positive, it is evident that, for a given $\bar{n}, d$ and $t / u$, the ground state solution can be found by finding the maximum kinetic (hopping) energy

$$
F=\langle V\rangle+(2 d-1)\left\langle X_{i}\right\rangle^{2}
$$

subject to the constraints

1. normalisation, $1=\langle s \mid s\rangle$

2. fixed mean occupation (per site), $\bar{n}=\frac{1}{2}\left\langle a_{1}^{\dagger} a_{1}+a_{2}^{\dagger} a_{2}\right\rangle$

3. fixed variance (per site), $\overline{n^{2}}=\frac{1}{2}\left\langle a_{1}^{\dagger} a_{1} a_{1}^{\dagger} a_{1}+a_{2}^{\dagger} a_{2} a_{2}^{\dagger} a_{2}\right\rangle$

In terms of the symmetric state (4.46) these constraints are respectively given by

$$
\begin{gathered}
\phi_{1}=\left(2 \sum_{N, p>0} C_{N, p}^{2}+\sum_{N} C_{N, 0}^{2}\right)-1=0 \text { (normalisation) } \\
\phi_{2}=\left(\sum_{N, p>0} C_{N, p}^{2} N+\frac{1}{2} \sum_{N} C_{N, 0}^{2} N\right)-\bar{n}=0 \text { (fixed mean) } \\
\phi_{3}=\left(\frac{1}{2} \sum_{N, p>0} C_{N, p}^{2}\left(N^{2}+p^{2}\right)+\frac{1}{4} \sum_{N} C_{N, 0}^{2} N^{2}\right)-\overline{n^{2}}=0
\end{gathered}
$$

Using the method of Lagrange multipliers, $F$ is then maximised when

$$
0=\frac{\partial F}{\partial C_{N, p}}-\frac{1}{2} \lambda \frac{\partial \phi_{1}}{\partial C_{N, p}}+\mu \frac{\partial \phi_{2}}{\partial C_{N, p}}+\nu \frac{\partial \phi_{3}}{\partial C_{N, p}}
$$

\section{Vectorising the problem}

We can write this set of simultaneous differential equations in matrix form when the set of coefficients $C_{N, p}$ is expressed as an ordered vector. To truncate the problem space in terms of a maximum two site occupation $N_{\max }$ (with a corresponding $p_{\max }$ ), it is convenient to use an ordering for the quantum numbers $p$ and $N$ with increasing $p$ (for each $N$ value) within increasing $N$. That is,

$$
\mathbf{C}=\left[C_{0,0}, C_{1,1}, C_{2,0}, C_{2,2}, C_{3,1}, C_{3,3}, C_{4,0}, C_{4,2}, C_{4,4} \ldots C_{N_{\max }, p_{\max }}\right]
$$

The correlation and mean-field can then be written respectively in terms of the vector quadratic forms

$$
\begin{aligned}
\langle V\rangle & =\mathbf{C}^{T} A_{V} \mathbf{C} \\
\langle X\rangle & =\mathbf{C}^{T} A_{X} \mathbf{C}
\end{aligned}
$$




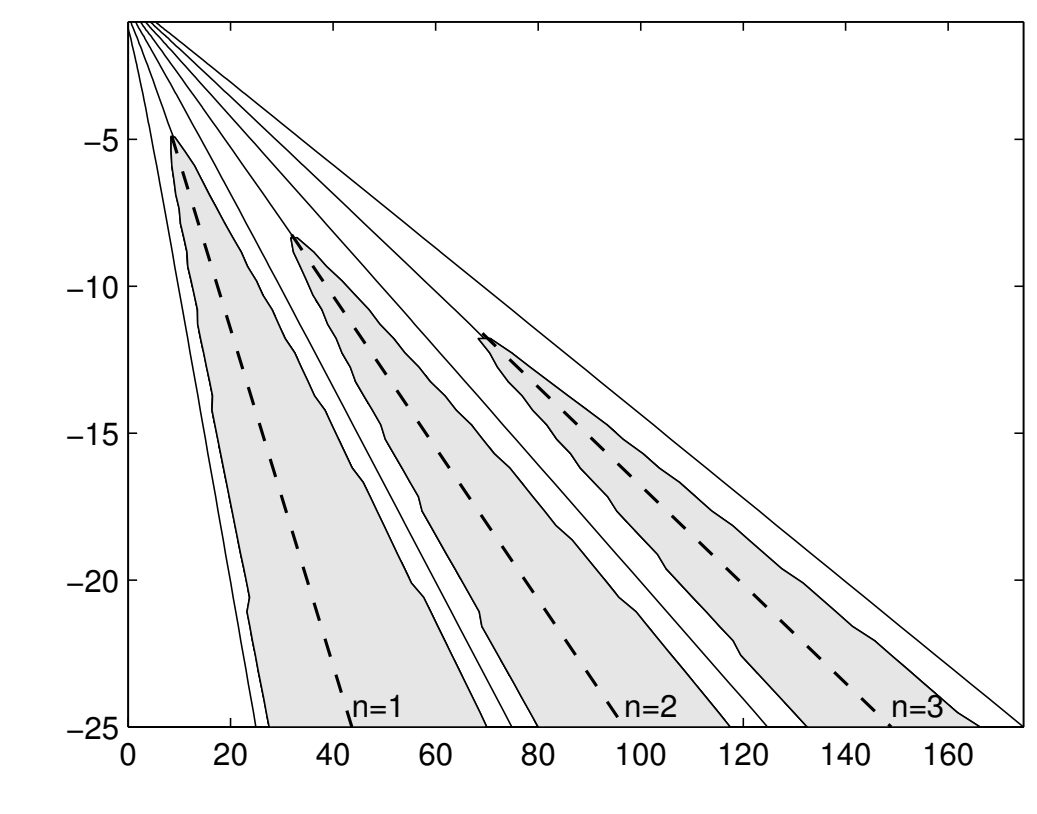

Figure 4.2: A contour plot of the mean occupation $\bar{n}$ with respect to the Lagrange multipliers $\mu$ and $\nu$ reveals the Mott lobes for commensurate occupations, shown for $\bar{n}=1,2,3$, where the solutions to (4.65) are degenerate with respect to $\mu$, indicating the incompressibility of the phase. The Mott insulator phase does not exist for incommensurate occupations as shown by the remaining curves at $\bar{n}=0.5,1.5,2.5$ and 3.5 from left to right in the figure. The dashed lines represent possible (non-degenerate) trajectories for the Mott insulator phase as discussed in section 4.2.1.

where the construction of matrices $A_{V}$ and $A_{X}$ follows directly from equations (4.14) and (4.45). In particular,

$$
A_{V}=\left(\begin{array}{c|ccccccccc}
0 & 0 & 0 & 0 & 0 & 0 & 0 & 0 & 0 & \\
\hline 0 & 1 & 0 & 0 & 0 & 0 & 0 & 0 & 0 & \\
0 & 0 & 0 & 2 \sqrt{2} & 0 & 0 & 0 & 0 & 0 & \\
0 & 0 & 0 & 0 & 0 & 0 & 0 & 0 & 0 & \\
0 & 0 & 0 & 0 & 2 & 2 \sqrt{3} & 0 & 0 & 0 & \\
0 & 0 & 0 & 0 & 0 & 0 & 0 & 0 & 0 & \\
0 & 0 & 0 & 0 & 0 & 0 & 0 & 2 \sqrt{6} & 0 & \\
0 & 0 & 0 & 0 & 0 & 0 & 0 & 0 & 4 & \\
0 & 0 & 0 & 0 & 0 & 0 & 0 & 0 & 0 & \\
\cline { 2 - 6 } & & & & & & & & & \ddots
\end{array}\right)
$$


and

$$
A_{X}=\left(\begin{array}{c|ccccccccc}
0 & 1 & 0 & 0 & 0 & 0 & 0 & 0 & 0 & \\
\hline 0 & 0 & 1 & \sqrt{2} & 0 & 0 & 0 & 0 & 0 & \\
0 & 0 & 0 & 0 & \sqrt{2} & 0 & 0 & 0 & 0 & \\
0 & 0 & 0 & 0 & 1 & \sqrt{3} & 0 & 0 & 0 & \\
0 & 0 & 0 & 0 & 0 & 0 & \sqrt{2} & \sqrt{3} & 0 & \\
0 & 0 & 0 & 0 & 0 & 0 & 0 & 1 & 2 & \\
0 & 0 & 0 & 0 & 0 & 0 & 0 & 0 & 0 & \\
0 & 0 & 0 & 0 & 0 & 0 & 0 & 0 & 0 & \\
0 & 0 & 0 & 0 & 0 & 0 & 0 & 0 & 0 & \\
& & & & & & & & & \ddots
\end{array}\right)
$$

The constraints (4.50)-(4.52) can then be written as

$$
\begin{gathered}
\phi_{1}=\mathbf{C}^{T} S \mathbf{C}-1=0 \\
\phi_{2}=\frac{1}{2} \mathbf{C}^{T} S D \mathbf{C}-\bar{n}=0 \\
\phi_{3}=\frac{1}{4} \mathbf{C}^{T} S D^{\prime} \mathbf{C}-\overline{n^{2}}=0
\end{gathered}
$$

where $S, D$ and $D^{\prime}$ are diagonal matrices with elements given by

$$
\begin{gathered}
S_{N N^{\prime} p p^{\prime}}=\delta_{N N^{\prime}} \delta_{p p^{\prime}}\left(2-\delta_{p 0}\right) \\
D_{N N^{\prime} p p^{\prime}}=\delta_{N N^{\prime}} \delta_{p p^{\prime}} N \\
D_{N N^{\prime} p p^{\prime}}^{\prime}=\delta_{N N^{\prime}} \delta_{p p^{\prime}}\left(N^{2}+p^{2}\right)
\end{gathered}
$$

with the usual Kronecker delta function. Using these definitions, equation (4.53) can then be written in matrix form as

$$
\left(S^{-1 / 2} A S^{-1 / 2}+S^{1 / 2} B S^{-1 / 2}\right) \mathbf{u}=\lambda \mathbf{u}
$$

where

$$
\begin{gathered}
\mathbf{u}=S^{1 / 2} \mathbf{C} \\
A=A_{V}+A_{V}^{T}+(2 d-1)\langle X\rangle\left(A_{X}+A_{X}^{T}\right) \\
B=\mu D+\frac{1}{2} \nu D^{\prime}
\end{gathered}
$$

This can be made linear in $\mathbf{u}$ (and therefore $\mathbf{C}$ ) when the mean-field term $\langle X\rangle$ in (4.67) is treated as a free parameter $x$; the resulting eigenvalue equation is solved iteratively in $x$ so that it is self-consistent with the calculated mean-field $\langle X\rangle=\mathbf{C}^{T} A_{X} \mathbf{C}$. The eigenvector solution $\mathbf{u}$ which maximises $F$ at each iteration is nonnegative; in fact the nonnegative eigenvector, the maximising solution, belongs to the maximum eigenvalue as we now show. 


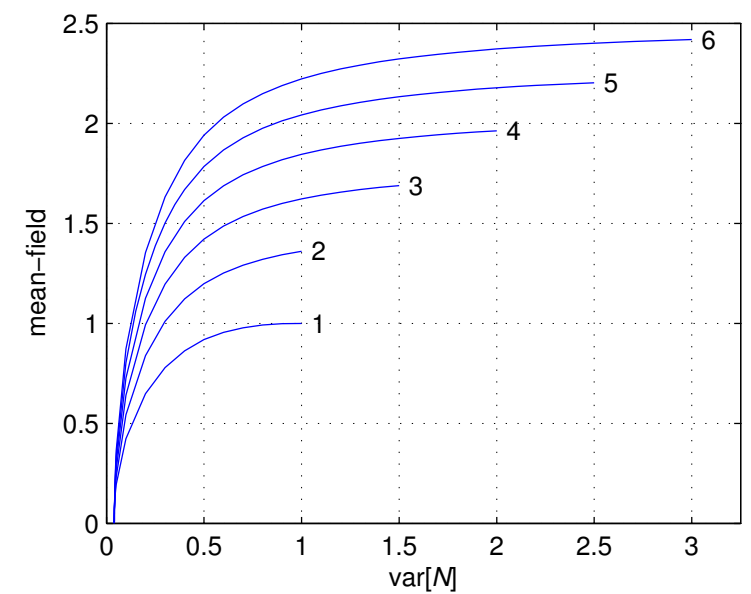

(a) Commensurate

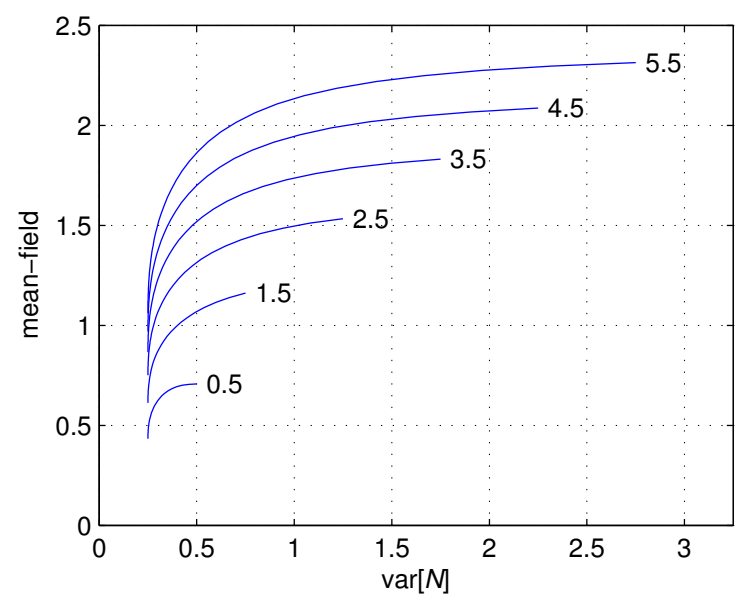

(b) Incommensurate

Figure 4.3: Mean-field as a function of number variance for commensurate (a) and incommensurate (b) occupations, both with $d=1$. In contrast to the one site approximation, in the commensurate case the Mott insulator phase with vanishing mean-field can occur for a non-zero number variance.

\section{Determining the maximising solution}

To see why this is the case we need to invoke the Perron-Frobenius theorem for nonnegative matrices [102]. We first note that the $r \times r$ matrix $G=S^{-1 / 2} A S^{-1 / 2}+S^{1 / 2} B S^{-1 / 2}$ in equation (4.65) is nonnegative everywhere except along the leading diagonal where negative values are possible when either $\mu$ or $\nu$ (or both) are negative. It is then always possible to construct a matrix $G^{\prime}=G+\gamma I_{r}$ that is nonnegative everywhere for a sufficiently large $\gamma, I_{r}$ being the $r \times r$ identity matrix.

The eigenvectors of $G^{\prime}$ and $G$ are clearly the same with the eigenvalues of $G^{\prime}$ given by $\lambda_{i}^{\prime}=\lambda_{i}+\gamma$ where $\lambda_{i}$ is the $i$ th eigenvalue of $G$ corresponding to the $i$ th eigenvector. The Perron-Frobenius theorem states that the spectral radius $\rho$ of a nonnegative matrix is an eigenvalue corresponding to a nonnegative eigenvector ${ }^{1}$. That is, $\rho\left(G^{\prime}\right)$ is the eigenvalue with the desired nonnegative solution. We can relate this to the maximum eigenvalue of $G$ when $\gamma$ simultaneously satisfies the following two conditions:

1. $\gamma \geq|\min (G)|$ so that $G^{\prime}$ is nonnegative

2. $\gamma \geq\left|\min \left(\lambda_{i}\right)\right|$ so that all $\lambda_{i}^{\prime} \geq 0$ and $\max \left(\lambda_{i}\right)+\gamma$ is necessarily the spectral radius of $G^{\prime}$

For any bounded matrix $G$ it is always possible to select such a value of $\gamma$; we can therefore associate the correct solution for $C_{N, p}$ with the maximum eigenvalue of (4.65) which returns a self-consistent mean-field.

\footnotetext{
${ }^{1}$ The spectral radius for an $n \times n$ matrix $A$ with eigenvalues $\lambda_{i}(1 \leq i \leq n)$ is defined as $\rho(A)=\max \left|\lambda_{i}\right|$.
} 


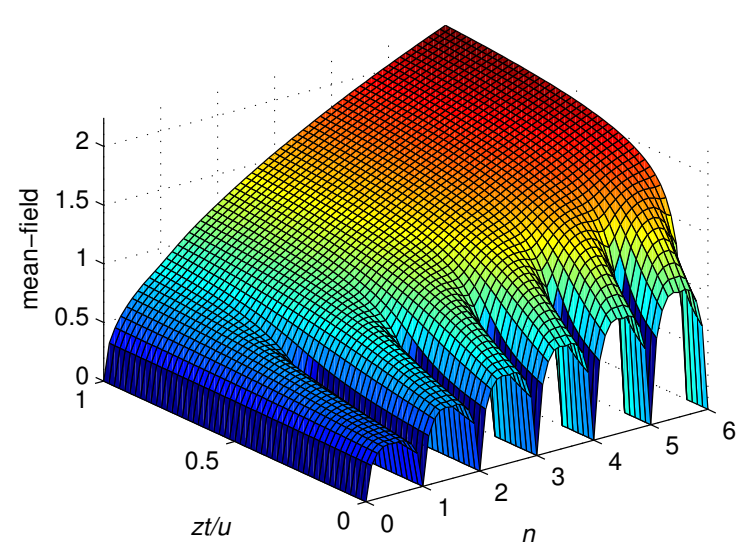

(a) Mean-field, $d=1$

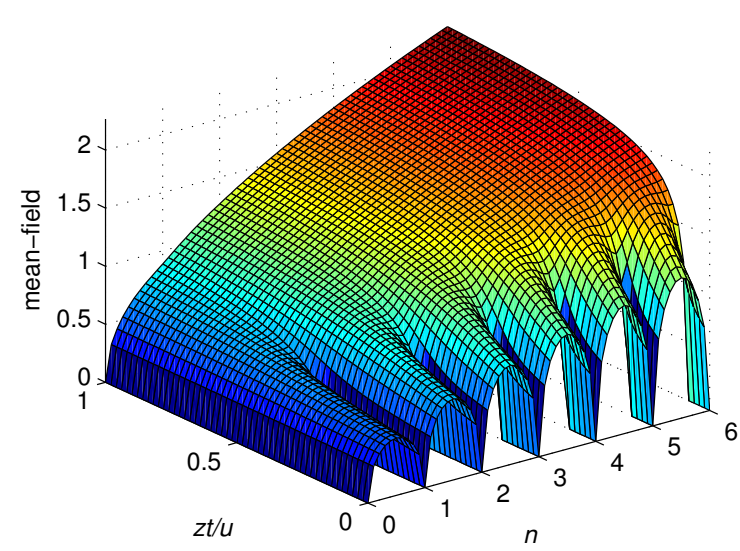

(c) Mean-field, $d=2$

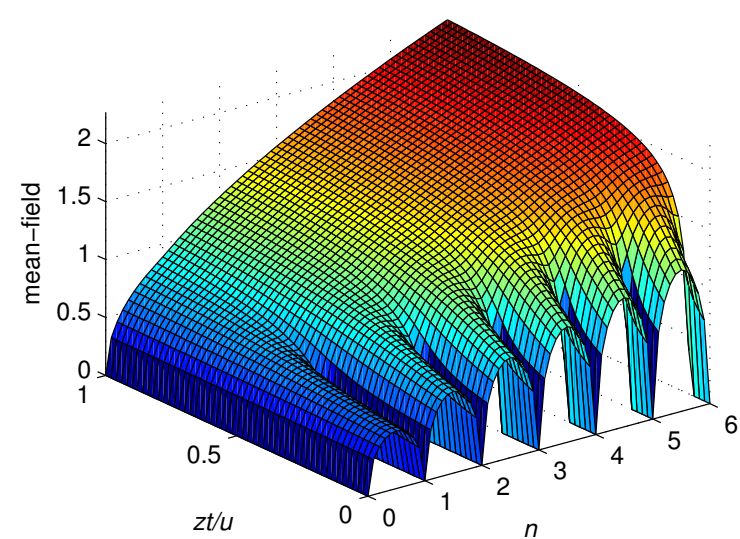

(e) Mean-field, d=3

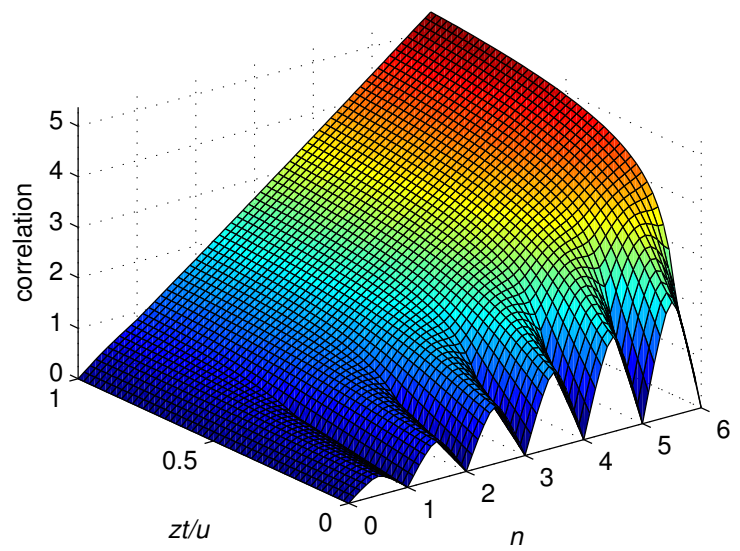

(b) Intersite correlation, $\mathrm{d}=1$

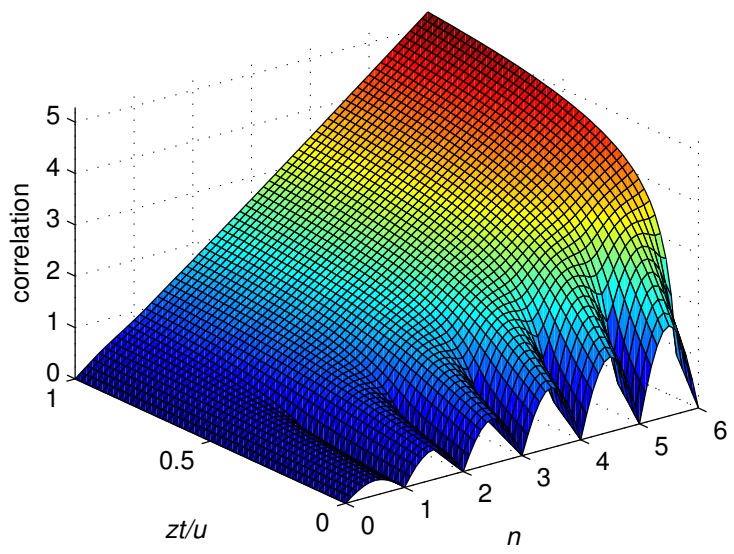

(d) Intersite correlation, $d=2$

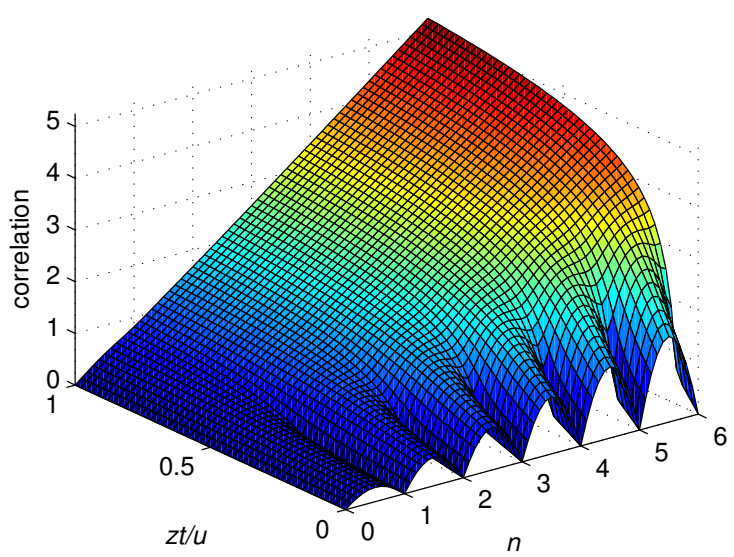

(f) Intersite correlation, $\mathrm{d}=3$

Figure 4.4: Ground state results for the Bose-Hubbard model in the two site mean-field approximation. The mean-field and intersite correlations are shown as a function of the mean site occupation $\bar{n}$ and the relative interaction strength $z t / u$. A non-zero mean-field represents the superfluid regime. The mean-field is zero below a critical value $(z t / u)_{c}$ for commensurate occupations, indicating the onset of the Mott insulator phase. The intersite correlations are non-zero everywhere except for commensurate occupations in the limit of no tunneling where $z t / u=0$. The results are qualitatively similar for 1, 2, and 3 dimensional lattices. However, a feature of the $d=1$ phase diagram is the weak suppression of the mean-field at half-integer occupations, which is seen more seen more clearly in Fig.4.6. This may be an artifact of the two site approximation and requires further study. 


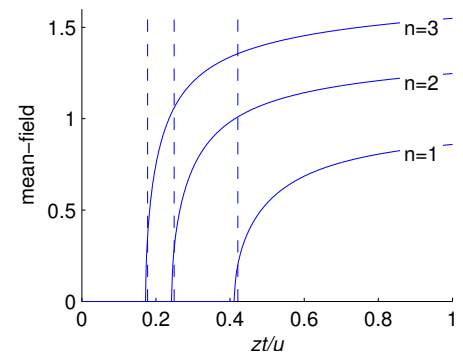

(a) Mean-field, $d=1$

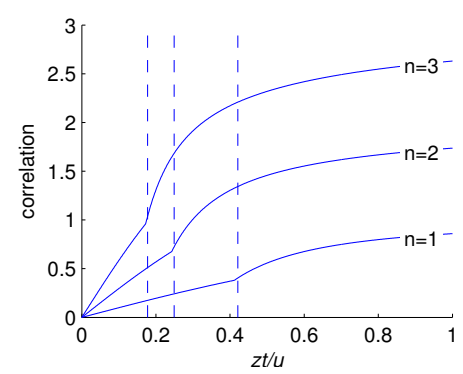

(d) Correlation, $d=1$

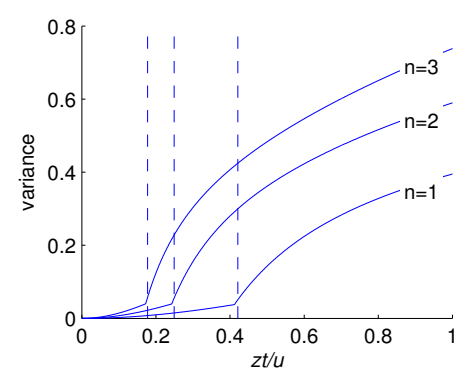

(g) Variance, $d=1$

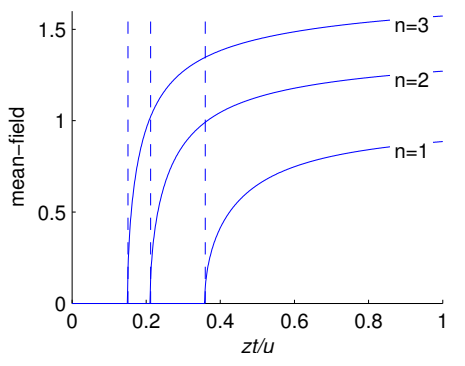

(b) Mean-field, $d=2$

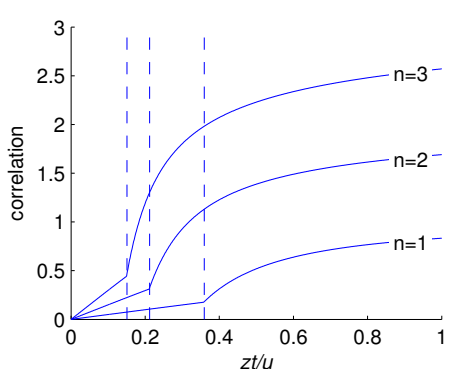

(e) Correlation, $d=2$

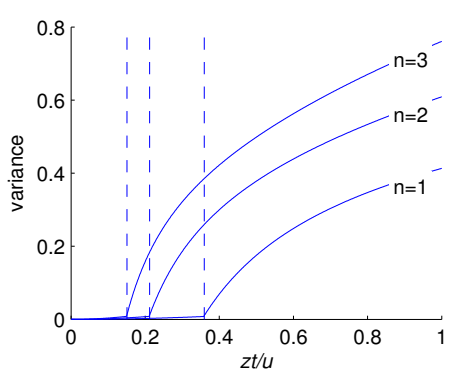

(h) Variance, $d=2$

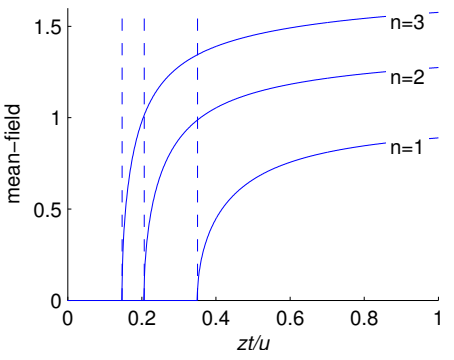

(c) Mean-field, $d=3$

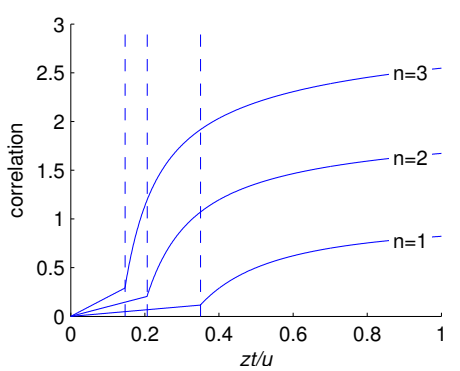

(f) Correlation, $d=3$

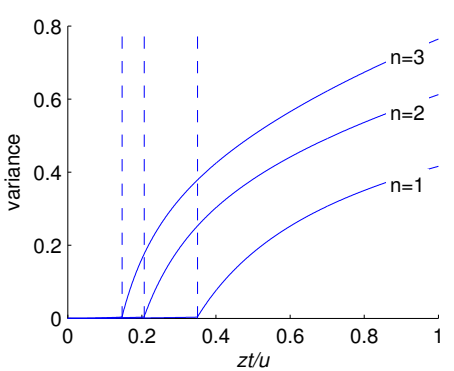

(i) Variance, $d=3$

Figure 4.5: $d=1,2,3$ results: mean-field, intersite correlation and variance for commensurate occupations of $\bar{n}=1,2,3$. The dashed vertical lines indicate the corresponding positions (from left to right) of the phase boundary between the superfluid and Mott insulator phases as calculated using the perturbation theory outlined in section 4.2 .3 


\subsubsection{The ground state solution}

The general procedure for finding the ground state solution follows the method used in the one site formulation (see section 3.2). In particular, at a given mean occupation $\bar{n}$, (4.65) determines a locus of solutions which can be expressed as a function of number variance. Therefore, at a given $t / u$ and $n$, the two site energy can be reparameterised in terms of number variance; finding the ground state phase diagram then only requires the minimisation of the energy with respect to variance. In practice to perform this procedure efficiently, it is useful to first calculate a set of solution states covering a suitable variance range, which act as initial conditions in the final minimisation step.

In the results presented here, this was achieved by fixing $\bar{n}$ and initially choosing a large value for the variance corresponding to the superfluid regime (specifically $\operatorname{var}[N]=\bar{n}$ for $\bar{n} \leq 1$ and $\operatorname{var}[N]=\bar{n} / 2$ for $\bar{n} \geq 1$ ). The corresponding state was found by solving equation (4.65) using the initial values $x_{0}=5, \mu_{0}=2$ and $\nu_{0}=-1$, which was found to be sufficient for the range of parameters considered here. A lower variance was then selected and its corresponding state was calculated, by using the previous solution to determine the initial conditions. By iteration it was then possible to find solutions efficiently over a broad variance range.

However, although this general procedure works well for most of the parameter space, it fails to converge (to a sufficient accuracy) in two limiting cases: for Mott-insulator states where the meanfield is zero and there is degeneracy; and in the limit of the lowest possible variance, $\operatorname{var}[N]=$ $\delta \bar{n}(1-\delta \bar{n})$, corresponding to the localised region where the Lagrange multipliers diverge at the solution. It is therefore necessary to consider these cases separately.

\section{Calculating Mott states}

For commensurate occupations and below a critical value for the variance, the system is in the Mott insulator state corresponding to a vanishing mean-field. In this case, the above approach is numerically unstable for two reasons. Firstly, the algorithm fails to correctly converge to a zero mean-field since the search direction cannot be determined. Secondly, the system states become highly degenerate in $\mu$ in the Mott insulator state (corresponding to a incompressible phase) which further prevents convergence (with respect to $\mu$ ).

These problems are circumvented respectively by the following adjustments to the general procedure. Firstly, explicitly setting the free-parameter $x$ to zero in the equation (4.65), ensures that any solution is self-consistent in the mean-field; this can be seen to be case by considering how the mean-field term appears in the eigenvalue equation (see (4.55) and (4.67) in particular). Secondly, setting $\mu=-r \nu$ for a suitably chosen quantity $r$, restricts the parameter space to the degenerate region, so that $\bar{n}$ remains fixed while $\operatorname{var}[N] \rightarrow 0$ in the limit $\nu \rightarrow \infty$. These trajectories are illustrated by the dashed curves in Fig.4.2. By enforcing this relationship between $\mu$ and $\nu$, we can therefore probe the Mott insulator states accurately. 


\section{Solutions in the limit of zero tunneling}

Considering (4.44) in the limit of zero tunneling where $t / u \rightarrow 0$, the ground state is clearly determined by states of the lowest possible variance. In this case, we found the method of Lagrange multipliers leads to poor convergence as the Lagrange multipliers diverge at the solution. We therefore consider a more direct method whereby we minimise the two site energy in terms of a reduced parameter space, which correspond to the lowest allowed variance $(\operatorname{var}[N]=\delta n(1-\delta n))$ for a given mean occupation $\bar{n}$.

Recall the lowest variance state is given by equation (4.36); in this basis, the hopping energy (4.49) is given by

$$
F=\beta / 2([\bar{n}]+1)\left(1+(2 d-1)(\sqrt{\alpha}+\sqrt{\gamma})^{2}\right)
$$

The constraints (normalisation, fixed $\bar{n}$ and fixed variance) can be given as a set of linear equations

$$
\begin{aligned}
\alpha+\beta+\gamma & =1 \\
{[\bar{n}] \alpha+\left([\bar{n}]+\frac{1}{2}\right) \beta+([\bar{n}]+1) \gamma } & =\bar{n} \\
{[\bar{n}]^{2} \alpha+\left([\bar{n}]^{2}+[\bar{n}]+\frac{1}{2}\right) \beta+\left([\bar{n}]^{2}+2[\bar{n}]+1\right) \gamma } & =\operatorname{var}[N]+\bar{n}^{2}
\end{aligned}
$$

which when written as $A x=b$, admits solutions of the form $x=x_{0}+\kappa$ nullspace $[A]$ where we have taken

$$
x_{0}=\left|\begin{array}{c}
\alpha \\
\beta \\
\gamma
\end{array}\right|=\left|\begin{array}{c}
{[\bar{n}]+1-\bar{n}} \\
0 \\
\bar{n}-[\bar{n}]
\end{array}\right|
$$

as a solution of the homogeneous system $A x=0$. There is an additional requirement on $\kappa$ that the coefficients are bounded with $0 \leq \alpha \leq 1,0 \leq \beta \leq 1$ and $0 \leq \gamma \leq 1$. For a given occupation $\bar{n}$ and dimension $d$, we can then maximise the hopping energy (4.69) with respect to the remaining free parameter $\kappa$. The ground state phase diagram is then calculated easily for the case where $z t / u=0$; the resulting mean-field and intersite correlation are shown in figures 4.6(a) and 4.6(b) respectively.

\section{Numerical Solutions}

Numerical calculations were performed using a state space truncated with $N \leq 20$, corresponding to 121 coefficients $C_{N, p}$. The MATLAB function f solve.m was used to find the self-consistent solution, for a given $\bar{n}$ and $\operatorname{var}[N]$, by supplying the function with suitable initial values $x_{0}, \mu_{0}$ and $\nu_{0}$ for the mean-field and Lagrange multipliers.

\subsubsection{Results}

Using the calculated states with maximum tunneling energy, the two site energy (4.44) was then minimised with respect to $\operatorname{var}[N]$ for a given $\bar{n}$ and $Z / u$. The results of this calculation are shown in Figs. 4.4(a)-(f) for arbitrary mean occupations. To investigate the superfluid to Mott-insulator transition more clearly, these results have also been reproduced for commensurate occupations in Figures 4.5(a)-(i). 


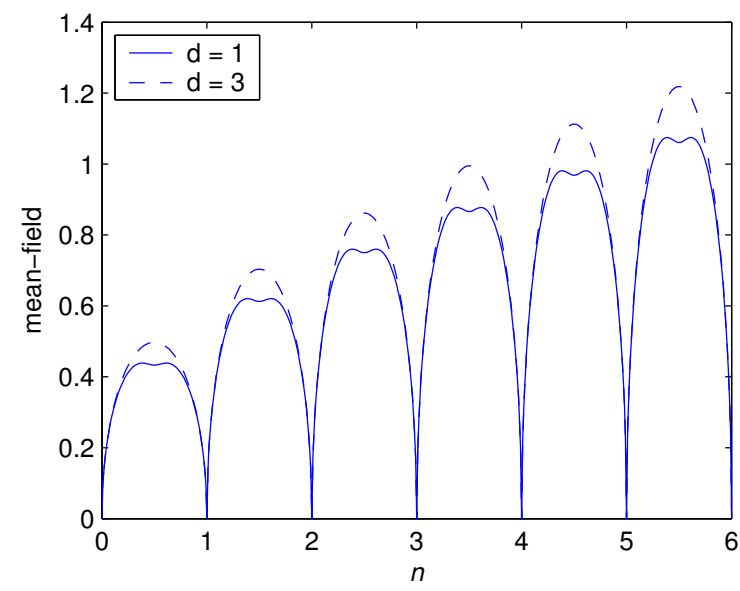

(a)

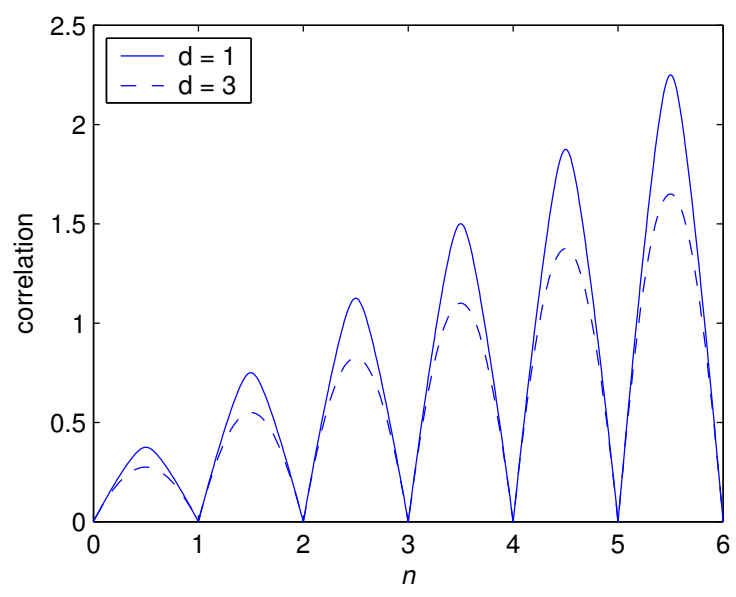

(b)

Figure 4.6: The mean-field (a) and intersite correlation (b) using a reduced basis of three states in the limit of zero tunneling $(z t / u=0)$ for $d=1,3$. The methodology is outlined in section 4.2.1. A feature of the $d=1$ case is the weak suppression of the mean-field around half-integer occupations. The $d=2$ results, which been omitted for clarity, are very close to the $d=3$ case.

This ground state phase diagram is qualitatively similar to the results for the one site model (Fig.2.2); the most important feature of both models is the vanishing of the mean-field for commensurate occupations above the critical transition point $\bar{u}_{c}$, corresponding to the Mott insulator phase. Note that the Mott insulator to superfluid transition is clearly reproduced for commensurate mean occupations, an improvement over the corresponding two site Q-function approximation (section 4.1) which can be attributed to the exact treatment of the intersite correlations in the present treatment. We now consider a simple perturbative expansion from which the position of the phase transition boundary can be easily calculated for the two site formulation.

\subsubsection{Calculating the phase transition boundary}

Following the one-site case, we consider the following states near the transition with commensurate occupation $\bar{n}$ :

$$
\begin{aligned}
|s\rangle= & \sqrt{1-\alpha-2 \beta}|\bar{n}, \bar{n}\rangle+\sqrt{\frac{\alpha}{2}}(|\bar{n}-1, \bar{n}+1\rangle+|\bar{n}+1, \bar{n}-1\rangle) \\
& +\sqrt{\frac{\beta}{2}}(|\bar{n}, \bar{n}-1\rangle+|\bar{n}-1, \bar{n}\rangle+|\bar{n}, \bar{n}+1\rangle+|\bar{n}+1, \bar{n}\rangle)
\end{aligned}
$$

This state is a superposition of the minimum set of number states that give a non-zero mean-field and intersite correlation. The coefficients have been chosen to satisfy normalisation and a fixed commensurate occupation $\bar{n}$. The corresponding variance on one site is given by $\operatorname{var}[N]=\alpha+\beta$. Note that $|s\rangle$ represents a perturbation in $\alpha$ and $\beta$ from the Mott insulator phase. With this state the intersite correlation (4.47) is given by

$$
\langle V\rangle=\sqrt{2(1-\alpha-2 \beta) \alpha} \sqrt{\bar{n}(\bar{n}+1)}+\frac{\beta}{2}(2 \bar{n}+1)
$$




\begin{tabular}{|l|ccc|}
\hline$d$ & $\bar{n}=1$ & $\bar{n}=2$ & $\bar{n}=3$ \\
\hline 1 & 4.75 & 8.02 & 11.26 \\
2 & 5.56 & 9.43 & 13.27 \\
3 & 5.71 & 9.69 & 13.63 \\
10 & 5.82 & 9.88 & 13.90 \\
$10^{6}$ & 5.83 & 9.90 & 13.93 \\
\hline
\end{tabular}

Table 4.1: Critical value $\bar{U}_{c}$ calculated for two site mean-field approximation using a perturbation expansion around the Mott Insulator state

and the mean-field (4.45) is

$$
\langle X\rangle=\sqrt{\frac{\beta}{2}}\left(\sqrt{1-\alpha-2 \beta}+\sqrt{\frac{\alpha}{2}}\right)(\sqrt{\bar{n}}+\sqrt{\bar{n}+1})
$$

In the ground state, the phase transition occurs for the minimum two site energy (4.44); clearly this occurs with respect to the state (4.72) when $\partial E_{12} / \partial \alpha=0$ and $\partial E_{12} / \partial \beta=0$ are both satisfied. Moreover the phase transition occurs for $\beta=0$ where the mean-field is zero. That is,

$$
\begin{aligned}
0=\left.\frac{\partial E}{\partial \alpha}\right|_{\beta=0}=\left(\frac{u}{t}\right)-\frac{\sqrt{\bar{n}(1+\bar{n})}(1-2 \alpha)}{\sqrt{2 \alpha(1-\alpha)}} \\
0=\left.\frac{\partial E}{\partial \beta}\right|_{\beta=0}=\left(\frac{u}{t}\right)-\frac{1}{2}(1+2 \bar{n}) \\
\\
-\frac{1}{2}(2 d-1)(\sqrt{\bar{n}}+\sqrt{\bar{n}+1})^{2}(\sqrt{1-\alpha}+\sqrt{\alpha / 2})^{2} \\
+\frac{\sqrt{2 \alpha \bar{n}(\bar{n}+1)}}{\sqrt{1-\alpha}}
\end{aligned}
$$

Numerically solving these equations leads to the results shown in table 4.1. Note that in the nonphysical limit of $d \rightarrow \infty$ the variance at the transition is $\operatorname{var}[N]=\alpha=0$. Considering equation (4.76) the transition then occurs at $\bar{U}_{c}=2(u / 2 d t)_{c}=(\sqrt{\bar{n}}+\sqrt{\bar{n}+1})^{2}$, the same value as in the one-site model. This is not unexpected as the intersite correlation $\langle V\rangle$ becomes negligible compared with the mean-field contribution in the Hamiltonian (4.1).

There are some notable differences between the one and two site mean-field approximations, which we discuss here. In particular, the inclusion of intersite correlations in the two site Hamiltonian, results in a non-zero number variance even in the Mott insulator phase where the mean-field vanishes; this can be seen in Figs. 4.3 and 4.5. This contrasts with the one site results (see Fig. 3.5) where the mean-field and number variance are identically zero. Moreover, for the two site model the calculated transition points $\bar{U}_{c}$, as shown in table 4.1, occur at lower values than for the one site model. In particular, for $\bar{n}=1$ and $d=1$, the two site model yields $\bar{U}_{c}=4.75$, whereas the one site model gives $\bar{U}_{c}=5.83$.

These differences are most pronounced for the $d=1$ case where the mean-field approximation 
is no longer valid, as has also been noted elsewhere [66]. At higher dimensions $(d=2,3)$, where the mean-field approximation is more accurate, the number variance is close to zero in the Mott phase and the calculated transition points for the one and two site models are close. It is expected that in the (non-physical) limit of infinite lattice dimensionality, the one and two site results will converge.

\subsubsection{Comparison to other work}

The results for the two site Hamiltonian given in the previous section clearly demonstrate a partial shift to lower values $\bar{U}_{c}$ (ie. a weaker lattice) when compared to the one site results. This shift can be attributed to the inclusion of intersite correlations in the model. To compare with our results with those reported elsewhere it is useful to refer to table 4.2.

For the $d=1$ case, numerically exact schemes, with Density Matrix Renormalisation Group (DMRG) and Quantum Monte-Carlo (QMC) simulations, predict a much lower value for the phase transition point $\bar{U}_{c}$ in the Bose-Hubbard model. Using the DMRG technique with the infinitesystem algorithm, the transition point for $\bar{n}=1$ and $d=1$ has been reported as $\bar{U}_{c}=1.68$ [94] and $\bar{U}_{c}=1.81$ [95]. Using the more accurate finite-system algorithm, the same transition has been reported as $\bar{U}_{c}=1.92$ [96] and $\bar{U}_{c}=1.68$ [97]. Similarly, for QMC simulations without lattice disorder, the transition has been reported as $\bar{U}_{c}=2.33$ [88]. Also, perturbative expansions using defect states have yielded similar results to the that of the QMC technique in one dimension. In particular, the above transition has been reported as $\bar{U}_{c}=2.33$ [82] and $\bar{U}_{c}=2.04$ [83].

\subsection{Conclusions}

We have presented two model Hamiltonians for the Bose-Hubbard model using two different mean-field approximations. The simpler one site model treats all intersite correlations using a mean-field approximation that decouples the problem into the sum of one site Hamiltonians. The second model is a two site extension where intersite correlations between two adjacent sites are explicitly included while treating interactions with neighbouring sites using the mean-field approximation. Each model has been tackled using two methodologies: a treatment in terms of a Q-function representation; and a numerically exact method using either the one or two site states.

In the case of the one site Hamiltonian, we find the Q-function representation agrees well with the numerically exact treatment, but can be solved at a fraction of the computational cost. For the two site Hamiltonian, the Q-function gives a good qualitative description but does not give a clear Mott insulator to superfluid transition due to limitations of the parameterisation on two sites. It is encouraging, however, that the Q-function approach gives the intersite correlations accurately when compared with the two site exact solution. In contrast to the Q-function approach, the two site exact solution yields a well-defined phase transition for commensurate mean occupations. In this case, the critical relative interaction strength $\bar{U}_{c}$ is smaller (corresponding to a weaker lattice strength) for all commensurate values of $\bar{n}$ than for the one site model; this shift is most pronounced for a one dimensional lattice.

What emerges from the Q-function approach is that the quantum mechanical constraints play 


\begin{tabular}{l|l|l|l}
\hline Method & $\begin{array}{l}\text { Position of phase transition } \\
\text { boundary for } d=1, n=1 \\
\text { (unless otherwise stated) }\end{array}$ & Authors & Reference \\
\hline $\begin{array}{l}\text { Mean-field approxima- } \\
\text { tion using second order } \\
\text { perturbation expansion }\end{array}$ & $\begin{array}{l}\bar{U}_{c}=2 n+1+\sqrt{(2 n+1)^{2}-1}, \\
\bar{n}=1: \bar{U}_{c}=5.83\end{array}$ & Oosten et al. & {$[68]$} \\
\hline $\begin{array}{l}\text { Mean-field theory using } \\
\text { variational approach }\end{array}$ & $\bar{U}_{c}=2.0$ & Amico and Penna & {$[80]$} \\
\hline $\begin{array}{l}\text { Mean-field theory using } \\
\text { variational approach }\end{array}$ & $\bar{n}=1: \bar{U}_{c}=5.83$ & Sheshadri et al. & {$[81]$} \\
\hline $\begin{array}{l}\text { Perturbative expansion } \\
\text { using defect (parti- } \\
\text { cle/hole) states }\end{array}$ & $\begin{array}{l}d=1, \bar{n}=1: \bar{U}_{c}=2.33 ; d=2, \\
\bar{U}_{c}=3.68\end{array}$ & Freericks and Monien & {$[82]$} \\
\hline $\begin{array}{l}\text { Perturbative expansion } \\
\text { using defect (parti- } \\
\text { cle/hole) states }\end{array}$ & $\begin{array}{l}\bar{U}_{c}=2.04 \text { (see table in refer- } \\
\text { ence for further calculated values } \\
\text { at different } d \text { and } n \text { ) }\end{array}$ & Freericks and Monien & {$[83]$} \\
\hline Quantum Monte-Carlo & $\bar{U}_{c}=2.33$ (without disorder) & Batrouni and Scalettar & {$[88]$} \\
\hline $\begin{array}{l}\text { DMRG with infinite- } \\
\text { system algorithm }\end{array}$ & $\bar{U}_{c}=1.68$ & Pai et al. & {$[94]$} \\
\hline $\begin{array}{l}\text { DMRG with infinite- } \\
\text { system algorithm }\end{array}$ & $\begin{array}{l}\bar{U}_{c}=1.81 \text { (without nearest- } \\
\text { neighbour interactions) }\end{array}$ & Kuhner and Monien & {$[95]$} \\
\hline $\begin{array}{l}\text { DMRG with finite- } \\
\text { system algorithm }\end{array}$ & $\begin{array}{l}\bar{U}_{c}=1.68 \text { (without nearest- } \\
\text { neighbour interactions) }\end{array}$ & Kuhner et al. & {$[97]$} \\
\hline $\begin{array}{l}\text { DMRG with finite- } \\
\text { system algorithm }\end{array}$ & \begin{tabular}{l}
$\bar{U}_{c}=1.92$ (for zero disorder) \\
\hline
\end{tabular} & Rapsch et al. & {$[96]$} \\
\hline
\end{tabular}

Table 4.2: The calculated boundary of the Superfluid to Mott-insulator phase boundary as determined from the Bose-Hubbard model as solved using various methods in the literature. Note, where appropriate, we have scaled the above values in terms of the variables defined in this work due to differing notation for $t$ and $U$ in some references. 
a primary role in determining the ground state results for the Bose-Hubbard model. This is most clearly illustrated by the one site formulation where the results are determined by the uncertainty relation in two forms: a restriction on the minimum variance permitted by the quantum state; and a relation between the number and phase fluctuations. In particular, the onset of the Mott insulator phase is characterised by suppression of number fluctuations at the lower bound of the variance constraint.

Exact numerical results with QMC or DMRG simulations on finite one dimensional lattices by other authors indicate the actual position of the superfluid to Mott insulator transition occurs for an even lower value of $\bar{U}_{c}$ than is calculated by our two site model. It is expected that if we were to extend our formalism to explicitly include the interactions between three or more sites, the predicted value $\bar{U}_{c}$ would shift to lower values in line with these other treatments. However, this remains a computationally difficult problem due to the dramatic increase in the size of the Hilbert space of the problem when more sites are included. 


\section{Part II}

An analogue model of an expanding universe in Bose-Einstein condensates 



\section{Chapter 5}

\section{Analogue models of gravity}

Though analogy is often misleading, it is the least misleading thing we have. Samuel Butler

\subsection{Introduction}

The unification of general relativity and quantum field theory into a theory of quantum gravity is a significant goal in theoretical physics, yet it remains elusive. Considerable progress has been made at the level of a semi-classical approximation, whereby quantum matter fields propagate in a fixed classical gravitational field; the applicability of the approximation is expected to be good at energy scales less than the Planck frequency where the background can be safely treated as a continuum. The resulting theory, known as quantum field theory in curved spacetime (QFTCS), predicts particle creation in strong gravitational fields. In particular, the prediction of thermal emission from a black hole by Hawking is arguably the most celebrated - and surprising - result to emerge from QFTCS. The experimental confirmation of the Hawking effect, however, remains a formidable task due to the enormous scales involved and the relatively small size of the effect. The development of analogue gravity models has therefore been motivated by the possibility of observing particle production in systems that could be realised in the laboratory, and so are amenable to experimental inquiry.

The connection between the propagation of sound waves in a fluid and quantum field theory in curved space was first made by Unruh in 1981 [103]. In this seminal publication, the analogue of Hawking radiation was shown to occur when a sonic horizon is formed in an inviscid irrotational fluid. In addition to numerous studies of these so-called sonic or acoustic black holes (see [27] and references therein), other analogue models of gravity have since appeared in the literature, including analogues of particle creation in expanding universes [104-111], and analogues for classical effects such as superradiance from rotating black holes [112,113]. The majority of such studies focus on fluid systems in either static or time-dependent geometries, which have an analogy with curved spacetime by the identification of an effective metric tensor. The propagation of sound waves in the fluid then has a close analogy with the propagation of a massless scalar field in a classical background.

The field of analogue gravity has lead to a large body of literature, including notably the 
appearance of two books $[114,115]$, several review articles $[27,116]$, and recently, a popular account for Scientific American [117]. Reviews specific to the case of acoustic black holes have also appeared $[118,119]$. We do not attempt a comprehensive review of the field here, and instead, refer the interested reader to the above references.

\subsection{Emergent spacetimes in Bose-Einstein condensates}

A particularly promising candidate system for analogue gravity models are Bose-Einstein condensates, a point which we will elaborate on in section 5.4. To motivate the discussion of analogue models, we show how the mean-field description of Bose-Einstein condensates leads an effective metric, which can describe a variety of emergent spacetimes.

\subsubsection{Establishing the analogy}

To see how analogue models of gravity emerge from the microscopic theory of Bose-Einstein condensates, we proceed following [27, 120].

The analogy between curved space and Bose-Einstein condensates can be revealed starting from the mean-field description of condensates, via the Gross-Pitaevskii equation (GPE):

$$
i \hbar \frac{\partial \Psi(\mathbf{x}, t)}{\partial t}=\left(-\frac{\hbar^{2}}{2 m} \nabla^{2}+V_{\mathrm{ext}}\right) \Psi(\mathbf{x}, t)+U|\Psi(\mathbf{x}, t)|^{2} \Psi(\mathbf{x}, t)
$$

The complex order parameter $\Psi(\mathbf{x}, t)$ can be written in terms of a real density and phase (known also as the Madelung representation) as

$$
\Psi(\mathbf{x}, t)=\sqrt{n(\mathbf{x}, t)} e^{i \theta(\mathbf{x}, t)}
$$

Substituting this into (5.1) and equating real and imaginary parts leads to the pair of equations

$$
\frac{\partial n}{\partial t}+\frac{\hbar}{m} \nabla \cdot(n \nabla \theta)=0
$$

and

$$
\hbar \frac{\partial \theta}{\partial t}-\frac{\hbar^{2}}{2 m} \frac{\nabla \sqrt{n}}{\sqrt{n}}+V_{\mathrm{ext}}+U n+\frac{\hbar^{2}}{2 m}(\nabla \theta)^{2}=0
$$

Identifying the background velocity for the irrotational field as

$$
\mathbf{v}=\frac{\hbar}{m} \nabla \theta
$$

we can rewrite (5.3) and (5.4) respectively as

$$
\frac{\partial n}{\partial t}+\nabla \cdot(n \mathbf{v})=0
$$


and

$$
\frac{\partial \mathbf{v}}{\partial t}+\mathbf{v} \cdot \nabla \mathbf{v}=-\frac{1}{m}\left(-\frac{\hbar^{2}}{2 m} \frac{\nabla \sqrt{n}}{\sqrt{n}}+V_{\mathrm{ext}}+U n\right)
$$

These equations are familiar from fluid dynamics; (5.6) is the continuity equation, whereas (5.7) is a modified form of Euler's equation.

Following $[27,120]$ we linearise about the fields $n$ and $\theta$ by setting

$$
\begin{gathered}
n \rightarrow n_{0}+n_{1} \\
\theta \rightarrow \theta_{0}+\theta_{1}
\end{gathered}
$$

Equations (5.3) and (5.4) then lead to

$$
\begin{gathered}
\frac{\partial n_{1}}{\partial t}=\frac{\hbar}{m}\left(n_{1} \nabla \theta_{0}+n_{0} \nabla \theta_{1}\right) \\
\hbar \frac{\partial \theta_{1}}{\partial t}-\frac{\hbar^{2}}{2 m} \widetilde{D}_{2} n_{1}+U n_{1}+\frac{\hbar^{2}}{m} \nabla \theta_{0} \nabla \theta_{1}=0
\end{gathered}
$$

where we have only retained terms up to first order in $n_{1}$ and $\theta_{1}$. The differential operator $\widetilde{D}_{2}$ has been introduced, which to first order, takes the form

$$
\widetilde{D}_{2} n_{1}=-\frac{1}{2} n_{0}^{-3 / 2}\left(\nabla^{2} \sqrt{n_{0}}\right) n_{1}+\frac{1}{2 \sqrt{n_{0}}} \nabla^{2}\left(\frac{n_{1}}{\sqrt{n_{0}}}\right)
$$

The operator $\widetilde{D}_{2}$ gives the first order correction from the inclusion of the quantum pressure term.

Rearranging (5.10) gives ${ }^{1}$

$$
n_{1}=-\hbar\left[U-\frac{\hbar^{2}}{2 m} \widetilde{D}_{2}\right]^{-1}\left(\frac{\partial \theta_{1}}{\partial t}+\mathbf{v} \cdot \nabla \theta_{1}\right)
$$

Substituting this into (5.9), to eliminate $n_{1}$ entirely, yields the second-order differential equation for the perturbed phase

$$
\begin{array}{r}
-\partial_{t}\left(\left[U-\frac{\hbar^{2}}{2 m} \widetilde{D}_{2}\right]^{-1}\left(\frac{\partial \theta_{1}}{\partial t}+\mathbf{v} \cdot \nabla \theta_{1}\right)\right) \\
+\nabla\left(\frac{n_{0}}{n} \nabla \theta_{1}-\mathbf{v}\left[U-\frac{\hbar^{2}}{2 m} \widetilde{D}_{2}\right]^{-1}\left(\frac{\partial \theta_{1}}{\partial t}+\mathbf{v} \cdot \nabla \theta_{1}\right)\right)=0
\end{array}
$$

This can be written in the compact form

$$
\partial_{\mu}\left(f^{\mu \nu} \partial_{\nu} \theta_{1}\right)=0
$$

\footnotetext{
${ }^{1}$ There is an assumption here, that the linear operator $T=U-\frac{\hbar^{2}}{2 m} \widetilde{D}_{2}$ is invertible, which is true only if the null space of $T$ is the zero vector space. This is certainly true for the homogeneous BEC as we will show in section 6.4.
} 
where we have introduced the symmetric matrix

$$
f^{\mu \nu}(\mathbf{x}, t)=\left[\begin{array}{ccc}
f^{00} & \vdots & f^{0 j} \\
\cdots & & \cdots \\
f^{i 0} & \vdots & f^{i j}
\end{array}\right]
$$

with components

$$
\begin{aligned}
f^{00} & =-\left[U-\frac{\hbar^{2}}{2 m} \widetilde{D}_{2}\right]^{-1} \\
f^{0 j} & =-\left[U-\frac{\hbar^{2}}{2 m} \widetilde{D}_{2}\right]^{-1} v^{j} \\
f^{i 0} & =-v^{i}\left[U-\frac{\hbar^{2}}{2 m} \widetilde{D}_{2}\right]^{-1} \\
f^{i j} & =\frac{n_{0}}{m} \delta^{i j}-v^{i}\left[U-\frac{\hbar^{2}}{2 m} \widetilde{D}_{2}\right]^{-1} v^{j}
\end{aligned}
$$

Note we have used the standard nomenclature where the Greek indices run from 0 to $d$ and the Roman indices from 1 to $d$ for $d$ spatial dimensions.

The equation for the phase fluctuations is formally analogous to the dynamics of a massless and minimally coupled scalar field in a curved space-time when (5.14) can be rewritten as [121]

$$
\frac{1}{\sqrt{-g}} \partial_{\mu}\left(\sqrt{-g} g^{\mu \nu} \partial_{\nu} \theta_{1}\right)=0
$$

where $g_{\mu \nu}$ is the effective metric tensor with determinant $g$. It is worth emphasising that although the external potential $V_{\text {ext }}$ does not explicitly appear here, the field equation still depends on $V_{\text {ext }}$ implicitly, as the the background geometry is determined by the stationary solutions of the GPE (5.1). We now show how the mapping to (5.17) is exact when the acoustic approximation is employed.

\section{The acoustic metric}

In the acoustic (or hydrodynamic) approximation the quantum pressure term is neglected. In this case $\widetilde{D}_{2}$ is set to zero and the resulting matrix $f$ takes the much simpler form

$$
f^{\mu \nu}(\mathbf{x}, t)=\frac{n_{0}}{c^{2}}\left[\begin{array}{ccc}
-1 & \vdots & -v^{j} \\
\cdots & & \cdots \\
-v^{i} & \vdots & c^{2} \delta^{i j}-v^{i} v^{j}
\end{array}\right]
$$

We have used the fact that the speed of sound in a condensate is given by $c^{2}=U n_{0} / m$.

This is known as either the hydrodynamic or acoustic approximation - in this case all collective excitations behave as sound waves with the usual linear dispersion form $\omega=c k-$ the 
quanta of excitations are thus phonons. An interesting consequence of Bogoliubov theory in BoseEinstein condensates is that in general the excitation spectrum displays nonlinear dispersion (see section 6.4), being linear (ie. phononic) for low $|\mathbf{k}|$ and becoming quadratic (ie. free-particle like) at large $|\mathbf{k}|$. When nonlinear dispersion is incorporated into analogue models of gravity it is equivalent to breaking Lorentz invariance [27]. We will return to this point in section 6.3.2.

The differential equation for the phase fluctuations given by (5.14) coincides with the field equation (5.17) when

$$
f^{\mu \nu}=\sqrt{-g} g^{\mu \nu}
$$

Since $\operatorname{det}\left(g^{\mu \nu}\right)=g^{-1}$ we can then write

$$
\operatorname{det}\left(f^{\mu \nu}\right)=-(-g)^{\frac{d-1}{2}}
$$

From (5.18) we also find directly that

$$
\operatorname{det}\left(f^{\mu \nu}\right)=-n_{o}^{d+1} c^{-2}
$$

Combining (5.20) and (5.21), we can therefore write

$$
\sqrt{-g}=n_{o}^{(d+1) /(d-1)} c^{-2 /(d-1)}
$$

and additionally using (5.17) we also have for the effective contravariant (ie. inverse) metric tensor

$$
g^{\mu \nu}(\mathbf{x}, t)=\left(\frac{n_{0}}{c}\right)^{\frac{-2}{d-1}} \frac{1}{c^{2}}\left[\begin{array}{ccc}
-1 & \vdots & -v^{j} \\
\cdots & & \cdots \\
-v^{i} & \vdots & c^{2} \delta^{i j}-v^{i} v^{j}
\end{array}\right]
$$

Inverting this gives the effective covariant metric tensor

$$
g_{\mu \nu}(\mathbf{x}, t)=\left(\frac{n_{0}}{c}\right)^{\frac{2}{d-1}}\left[\begin{array}{ccc}
-\left(c^{2}-v^{2}\right) & \vdots & -v_{j} \\
\cdots & & \cdots \\
-v_{i} & \vdots & \delta_{i j}
\end{array}\right]
$$

The corresponding line element for this geometry is

$$
d s^{2}=\left(\frac{n_{0}}{c}\right)^{2 /(d-1)}\left[-\left(c^{2}-v^{2}\right) d t^{2}-2 \mathbf{v} \cdot \mathbf{d} \mathbf{x} d t+\mathbf{d} \mathbf{x}^{2}\right]
$$

This is in the Painlevé-Gullstrand form up to a conformal factor ${ }^{2}$. Note that when there is no background flow $(\mathbf{v}=0)$ and the spacetime geometry is time-independent, (5.24) reduces to the metric for a flat Minkowski spacetime (up to a conformal factor).

\footnotetext{
${ }^{2}$ For a discussion on the relationship between Painlevé-Gullstrand coordinates and the better known Schwarzschild coordinate system see Visser [118].
} 


\section{Effective field theory}

Equation 5.17 is the massless Klein-Gordon equation. The metric (5.24) has the signature $(-+++)$ and so the effective spacetime represents a Lorentzian geometry; that is, the massless Klein-Gordon equation is invariant to Lorentz transformations. It is interesting to note that a relativistic wave equation arises from the equations of motion for a non-relativistic fluid.

The hydrodynamic description (ie. long wavelength) description of a BEC leads to an effective field theory (EFT) for curved spacetime in the same sense that classical gravity is expected to emerge as the low-energy EFT of a full theory of quantum gravity [122]. (The analogue gravity EFT arising from a BEC does not impose a strict cut-off and so includes so-called trans-Planckian physics - we return to this point in section 6.3.2). However, it should be noted that the emergent spacetime is itself embedded in an low-energy EFT for the Bose system; here the cut-off is determined by the validity of replacing the two-body interaction potential by an effective potential that is characterised by the s-wave scattering length. In practice, the cut-off is enforced by the the use of a projector in mode space (see chapter 7).

We further emphasise that the above analogy only holds for massless spin- 0 particles; in general, it is possible to modify the formalism to include massive modes at the expense of dealing with a more complex configuration, e.g. a two-component BEC [123]. Moreover, it is possible to develop an analogue model of gravity for spin-1 fields, eg. in dielectric media [124] (see section 5.4 for more on dielectric models of analogue gravity).

\section{Dynamics and Kinematics}

The analogue gravity framework we have thus far established is able to reproduce many of the kinematic aspects of general relativity; in particular, quantum field theory in curved spacetime leads to the notion of particle production, as described by the Hawking effect for example. The degree to which we can extend the analogy to dynamical aspects of general relativity is not clear. Here, by "dynamical", we mean a description of the evolution of the spacetime geometry itself, as is governed by Einstein's equations in general relativity. In the analogue context, the effective metric arises from the physical theory of the underlying fluid, and there is not necessarily any dependence on Einstein's equations or analogue thereof. This point of view has been emphasised by Visser $[118,125]$. However, there have been some efforts to further probe the analogy, of which we mention three:

Lagrangian models: Barcelo et al. [126] have shown that the construction of the Lagrangian model (and corresponding action) for an acoustic geometry contains a term that is proportional to the Einstein-Hilbert action, which arises from one-loop effects and the idea of Sakharov's “induced gravity”.

Ermakov system: Lidsey showed that a correspondence exists between a four-dimensional Friedmann-Robertson-Walker spacetime and a two-dimensional Bose-Einstein condensate in a harmonic trap, because both systems can be mapped to a one-dimensional Ermakov system [127]. 
Two-dimensional dilaton gravity: Cadoni has shown that it is possible to map a spherically symmetric acoustic black hole to a two-dimensional dilaton gravity model, and therefore define a mass, temperature and entropy for the system [128].

A related issue is the back-reaction of the quantum field on the geometry, which has been neglected in the linearised theory above. In fluid analogues, the phononic excitations and effective spacetime are both described by the microscopic theory of the constituent matter; for example, for analogue models in Bose-Einstein condensates, the quantum theory of the underlying bosonic atoms should be sufficient - in principle - to describe the kinematics and dynamics of the system, including the effects of back-reaction.

The ability to derive an effective metric tensor does not automatically assure that the spacetime is a stable quantity. After all, this picture neglects the dynamical evolution of the system. The stability of the geometry should be checked by finding the normal modes of the effective field theory, by including the effects of back-reaction, Lorentz violation and any external forces that may be present.

\section{The role of dimensionality}

It is interesting to comment on the role of dimensionality at this point. Clearly the metric tensor (5.24) is ill-defined for $d=1$ and so we surmise there is no analogue model of gravity (or effective spacetime) for this case, even though (5.14) adequately describes the dynamics of the system.

However, this is not the end of the story: it is still possible to model a $(d+1)$ dimensional spacetime using an effectively lower dimensional description of the system by using dimensional reduction; that is, by utilising the symmetries of the spatial geometry to integrate out one or more degrees of freedom. This is a valid approach so long as the reduced description does not neglect any of the essential physics of the original system, such as topological excitations (eg. rotons or vortices).

In particular, in part III of this thesis, we investigate an acoustic black hole model in a ring geometry by using a $(1+1)$ dimensional formulation; in this case, we implicitly make use of symmetry in the transverse dimensions to justify the reduced description.

\section{Horizons and geometric acoustics}

The effective metric (5.24) derived above, can be used to describe a variety of emergent spacetime geometries. In particular, consider the case when the geometry is stationary (by making $n_{0}, c$ and $v$ independent of time). If there exists a closed surface ${ }^{3}$ where the flow becomes supersonic, being subsonic on one side and supersonic on the other, the system is said to have a sonic horizon; sound waves originating in the supersonic region cannot propagate against the flow, and the sound waves are thus "trapped" in the supersonic region.

A sonic horizon is an example of a trapped surface [118]. In particular, an outer trapped surface is a closed surface where the fluid velocity is everywhere ingoing and the normal component is supersonic at the surface, so that a sound wave necessarily propagates inwards. An outer trapped

\footnotetext{
${ }^{3}$ In general this surface has spatial dimension $d-1$
} 


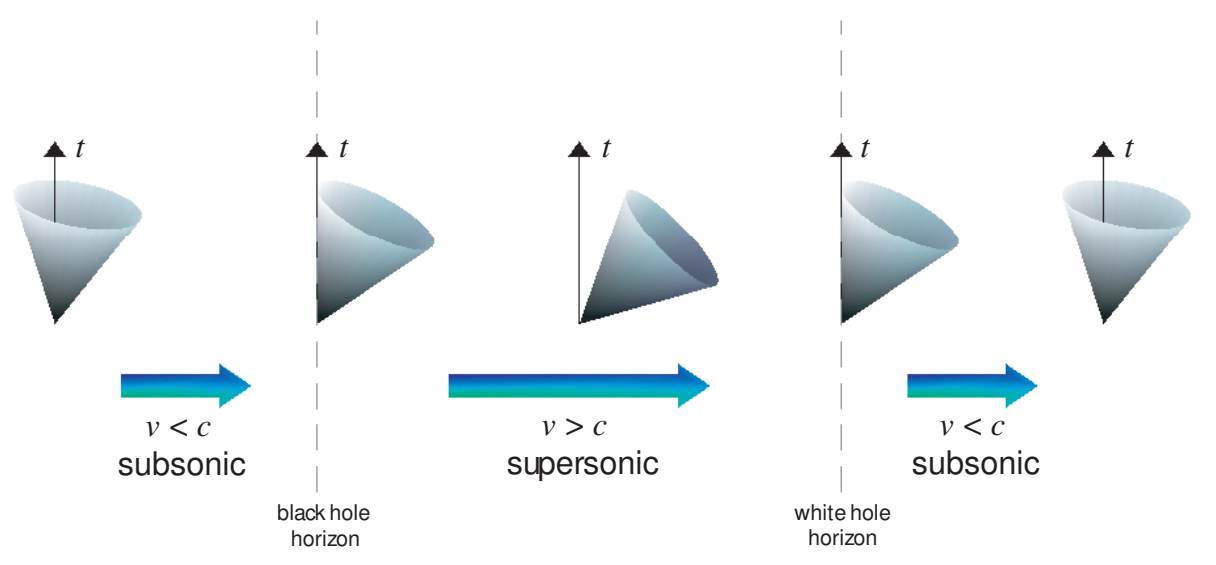

Figure 5.1: Sonic horizons in a flowing fluid: the black and white hole horizons occur where the normal component of the fluid velocity is equal to the local speed of sound; between the two surfaces, a region called the ergoregion, the flow is supersonic. The behaviour of sound waves at different points in the fluid flow is shown by "sound cones" (the wavefronts of sound waves emitted from a point, defining the locus of null geodesics); the sound cones are tipped in the direction of flow in the supersonic region so that all null geodesics necessarily propagate in the direction of the flow. See the text for more details.

surface behaves like a black hole horizon, with the interior/exterior of the hole given by the supersonic/subsonic region. In contrast, for an inner trapped surface the fluid velocity is everywhere outgoing with the normal component supersonic; sound waves must propagate outwards. Therefore, an inner trapped surface behaves like a white hole horizon. Note that a white hole horizon is the time-reversed case of a black hole horizon. The presence of both outer and inner trapped surfaces (ie. sonic horizons) for a fluid flow is illustrated in Figure 5.1. A variety of analogue spacetimes with sonic horizons has been analysed by Barcelo et al. [129].

This all follows naturally from the idea of geometric acoustics, which is discussed in detail elsewhere $[118,130]$. The propagation of sound waves is governed by the null geodesics of the metric tensor, given by

$$
d s^{2}=g_{\mu \nu} d x^{\mu} d x^{\nu}=0
$$

which yields the ray equation

$$
\left\|\frac{d \mathbf{x}}{d t}-\mathbf{v}\right\|=c
$$

The norm has been taken in Euclidean space. That is, a sound wave (ie. phonon) propagates with speed $c$ relative to a flow velocity $v$. The geodesic description is restricted to short wavelength modes (ie. it describes the propagation of classical test particles in a spacetime), and so, it is not strictly valid as a physical description of our system where the short wavelength modes fall outside the acoustic regime. However, we retain the geodesic description, as it usefully connects the concepts of sonic horizons to event horizons that appear in general relativity. 
In fact, a more rigorous consideration of spacetime geometries leads to a refinement of these ideas. We note in particular the following cases for trapped surfaces (see, for example $[118,130$, 131]):

Event horizon: the surface that separates regions where null geodesics can or cannot escape to infinity. This definition depends on the entire future history of the universe and so is a global property of the spacetime. Because the acoustic analogue reproduces the kinematic aspects of gravity, the notion of an (absolute) event horizon here is not strictly applicable, unless our model also includes a description of the dynamical evolution of the system. For this reason, it is more appropriate to consider apparent horizons.

Apparent horizon: the outermost closed surface on which all outgoing future-directed null geodesics have zero expansion. Inside the surface (ie. within the trapped region) outgoing null geodesics are converging; that is, the wavefronts of sound waves are decreasing in area. The concept of an apparent horizon is useful for acoustic geometries as it can be defined instantaneously; it is a local property of the spacetime. In a stationary geometry, however, the event horizon and apparent horizons are identical.

Ergoregion: the region of space where the timelike Killing vector $\partial_{t}$ is spacelike. Thus the boundary of the ergoregion - the ergosphere - occurs where $g_{00}=0$. In a cosmological context, an ergoregion is any region where an observer cannot remain stationary relative to the background; this concept is particularly relevant to rotating black holes (eg. Kerr black holes) where the tangential velocity of the black hole may exceed the speed of light. For an acoustic analogue, any region where the flow is supersonic is an ergoregion. In general, the ergoregion and apparent horizon do not coincide; however, for a $(1+1)$ dimensional spacetime the ergoregion and apparent horizon are always the same.

In what follows, we consider quasi-stationary geometries; that is, we cannot formally define an event horizon for the system, but apparent horizons may certainly exist.

\subsection{Models of Analogue Gravity}

In this thesis we consider two classes of models that can be described by the effective metric (5.24): These are analogue models for cosmological and black hole geometries.

\subsubsection{Cosmological Models}

\section{Expanding universe models}

The prediction of particle production in an expanding universe originates from Parker's work in the 1960's [132-134]. It is routinely believed that the large scale structure in the present universe (eg. cosmic background radiation) may have therefore occurred during a period of rapid inflation of the early universe. Accordingly, an important class of cosmological models are Friedmann- 
Robertson-Walker (FRW) spacetimes ${ }^{4}$; these are geometries that are described by the metric

$$
d s^{2}=-c^{2} d t^{2}+a_{\mathrm{FRW}}^{2}(t) d S^{2}
$$

where $a_{\mathrm{FRW}}$ is the scale factor of the universe (we later introduce a related scale factor $b(t)$ ) and $d S^{2}$ is the line element for three-dimensional space that is both homogeneous and isotropic. In general there are three possibilities for the spatial geometry: (i) flat; (ii) closed (spherical) and (iii) open (hyperbolic). In what follows we consider only the case where the spatial geometry is flat; there are two analogue models of an expanding universe in Bose-Einstein condensates that can reproduce a flat FRW metric:

Physical expansion: A number of investigations have considered the formulation of an analogue FRW cosmology based on the free expansion of a BEC [104-109, 135]. In this configuration, the BEC is initially confined to a harmonic trap, having a Thomas-Fermi profile in the hydrodynamic approximation. By a choice of suitable co-moving coordinates (by a scaling transformation as in [136]), it is then possible to express the metric in the form (5.28), at least approximately.

Effective expansion: An alternative - and conceptually simpler - scenario involves using a time-dependent speed of sound, while the background remains stationary (ie. $\mathbf{v}=0$ ) [109-111,135]. In BECs this would be achieved in practice by tuning the atomic interactions via a Feshbach resonance $[137,138]$.

We elaborate on these models in chapter 6, and in particular, focus on the case of an effective expansion, which we investigate in detail.

\section{Bose nova}

An example of a process that can be interpreted within the framework of cosmology and QFTCS, but which bears no resemblance to any specific cosmological system, is the phenomenon of condensate collapse now commonly referred to as a Bose nova. The collapse of a Bose-Einstein condensate was initially observed by Donley and co-workers by switching the interactions of a condensate of ${ }^{85} \mathrm{Rb}$ atoms from a repulsive to attractive via a Feshbach resonance [139]. For a scattering length $a$, this corresponds to a signature change from a Lorentzian signature (for $a>0$ ) to an Euclidean signature (for $a<0$ ). The characteristic feature of this effect is the emission of atoms from the condensate by bursts of atoms and jet formation. Calzetta and Hu have subsequently analyzed this effect in the context of cosmology, as squeezing and parametric amplification of vacuum fluctuations [140,141]. It is interesting to note that this is an example of an unstable system.

\subsubsection{Acoustic Black Hole Models}

The initial proposal by Unruh [103], and most subsequent efforts in analogue gravity, have focused on the case of acoustic black hole models. We have already introduced the idea of sonic horizons

\footnotetext{
${ }^{4}$ Also called Friedmann-Lemaître-Robertson-Walker spacetimes.
} 
for fluid models in section 5.2.1; the effective metric (5.24) can exhibit sonic horizons if the flow becomes supersonic $(v>c)$ in some region.

We discuss a number of possible configurations for the formation of sonic horizons in fluid systems, with particular emphasis on Bose-Einstein condensates.

\section{Nozzles}

A simple realisation of a sonic horizon can be made using a nozzle; this is implemented by a constriction of fluid flowing through some region so that the fluid accelerates. In particular, the most suitable example is the de Laval nozzle which usually appears in the context of rocket propulsion. A de Laval nozzle is formed by a converging-diverging constriction. Simple considerations from fluid mechanics show that as the fluid approaches the waist of the nozzle, the fluid accelerates. Under suitable conditions the fluid velocity may exceed the local speed of sound as it exits the waist of the nozzle; the position of the sonic horizon, which is formed, adjusts itself to occur at the waist of the nozzle.

In a normal fluid a de Laval nozzle could be formed by a physical constriction in a tube through which the fluid flows. In a Bose-Einstein condensate it could be formed by an external confining potential for example.

The possible use of a de Laval nozzle to form sonic horizons has been previously investigated $[114,135,142-145]$. The stability of a system with sonic horizons may require a pair of such nozzles so that the region of supersonic flow is bounded on each side by a region of subsonic flow [135, 142]. We will introduce a similar geometry in part III of this thesis (section 9.2), where we develop a specific model of an acoustic black hole in Bose-Einstein condensates.

The boundary conditions of a nozzle geometry have a major influence on the resulting physics, particularly in quantum systems such as Bose-Einstein condensates. In our model, we consider a one-dimensional system with periodic boundary conditions, so that the geometry is toroidal (ie. a ring system). This leads a non-trivial topology that exhibits a fixed winding number, similar to the situation found in vortex geometries. Other nozzle models however, may use open or absorbing boundary conditions, so that the flow can violate topological constraints [146,147]. We return to this point in chapter 9 .

It is also worth commenting on the so-called critical black hole configuration. In this scenario, the flow becomes sonic at a single point only, so that the system lacks an ergoregion. A recent investigation by Barcelo et al. has shown that, in principle, a system that generates a critical black hole in an analogue model - in either a finite time or in the asymptotic future with "sufficient rapidity" — should also exhibit the Hawking effect with a Planckian spectrum [27].

\section{Vortex geometries}

Another class of acoustic black holes are described by vortex geometries $[112,113,118,148]$. In general, such a geometry consists of a fluid with both radial and tangential velocity components with a sink at the origin. An outer trapping surface is formed if the ingoing radial velocity exceeds the local speed of sound inside some surface. This is the analogue of an event horizon as the fluid 
is removed at the sink. Conversely, the surface where the tangential velocity exceeds the speed of sound defines an ergoregion.

Vortex geometries with rotation are analogous to the case of rotating black holes (ie. Kerr black holes). As such, in addition to Hawking radiation, the classical phenomenon of superradiance should be observable in such systems. Theoretical investigations of fluid systems have confirmed that superradiant scattering of sound waves occurs in vortex geometries $[112,148]$. A more recent analysis has focused on the case of Bose condensed systems [113]. In that work the hydrodynamic approximation was used and so the background flow was treated classically. However, a full quantum treatment of superradiant sound scattering in acoustic black holes in Bose-Einstein condensates has not yet been undertaken.

The case where there is only a radial velocity component still exhibits a sonic horizon, and therefore also the possibility of Hawking radiation. This situation been investigated for the case of Bose-Einstein condensates in a one dimensional configuration [149].

\section{Propagating phase boundaries}

Recently it has been suggested that a sonic horizon could also be formed by a shifting phase boundary [150]. In this scenario, there are two stable phases for the fluid, with the system initially prepared in the first of these phases. Under suitable circumstances, a bubble of the second phase can nucleate and grow forming a propagating phase boundary at the interface of the two phases, which acts as a sonic horizon for the second phase.

\subsubsection{Classical and Quantum systems}

Broadly speaking, physical systems for analogue gravity can be divided into two types:

- Classical systems: both the background geometry and field fluctuations are taken to be classical quantities. This is the case for normal fluids or models based on electrodynamics.

- Quantum systems: both the background geometry and field fluctuations are quantised. This is the case for quantum fluids such as Bose-Einstein condensates or superfluid Helium, or for slow light analogues in dielectric media.

In fact, it should in principle be possible to observe Hawking radiation in classical systems. In particular, the classical counterpart of Hawking radiation in a de Laval nozzle has been shown to also occur $[143,145]$. Additionally, the classical properties of an acoustic black hole formed by a de Laval nozzle has been explored by Cardoso [144].

In the majority of studies of interacting quantum systems, a common approximation that is employed is to use a semi-classical model where the background geometry is classical whereas the field fluctuations are quantised. This is the same situation considered in the normal view of quantum field theory in curved space. In part III of this thesis, we undertake a quantum treatment of an acoustic black hole configuration in a Bose-Einstein condensate, which leads to interesting modifications to the semi-classical result. 


\subsection{Systems for Analogue Gravity}

There are a number of specific systems that are candidates for analogue models of gravity. We provide a brief overview of the most prominent of these systems, noting that a more comprehensive review has been undertaken elsewhere [27].

\subsubsection{Superfluid Helium}

The possibility of using superfluid Helium for analogue models of gravity has received considerable attention (see [115] and references within). Superfluid Helium occurs as both fermionic ${ }^{3} \mathrm{He}$ and bosonic ${ }^{4} \mathrm{He}$ isotopes, for which analogue models are available.

In particular, the formation of black and white hole pair by a soliton propagating faster than the local speed of sound in superfluid ${ }^{3} \mathrm{He}-\mathrm{A}$ has been considered by Jacobson and Volovik [151,152]. Moreover, it is also possible to form an effective $(2+1)$ dimensional spacetime at the interface between two superfluids on which surface waves (known as ripplons) can propagate [153]. The formation of sonic horizons at the interface of the ${ }^{3} \mathrm{He}-\mathrm{A}$ and ${ }^{3} \mathrm{He}-\mathrm{B}$ superfluid phases has been investigated by Volovik $[154,155]$.

However, the analysis of these systems is complicated by the fact that superfluid Helium is a strongly interacting system, and a mean-field description is difficult to formulate. This is compounded by the fact that Landau instabilities can occur for flow velocities smaller than the speed of sound, due to the existence of roton minima. Thus it may be very challenging to experimentally construct stable sonic horizons in these systems.

\subsubsection{Shallow water waves}

A particularly simple system, which leads to an effective spacetime description, is that of surface waves (gravity waves) in a shallow basin filled with a liquid, as proposed by Schützhold and Unruh [156]. They showed that a viscosity free, incompressible and irrotational fluid in a shallow basin leads to an effective metric for long wavelength waves (specifically, the wavelength is much longer than the basin depth). When the analysis includes an arbitrary flow velocity and basin depth, the effective spacetime is curved and supports black and white hole horizons. Although this system is not suitable for exploring quantum effects such as Hawking radiation, it can be used to investigate classical aspects of black holes, such as instabilities due to the mixing of positive and negative norm modes at a horizon.

\subsubsection{Slow light in dielectrics}

A quite different system, which leads to an effective spacetime description, is that of "slow light" in dielectric media [124,157-159]. The basic idea is to make use of Electromagnetically-Induced Transparency, which can dramatically reduce the group velocity of light pulses in a suitable material. When in addition the medium is moving, this leads an effective metric that can emulate curved spacetime geometries.

However, Unruh and Schutzhold [160] have shown that although slow light analogues can 
reproduce classical aspects of black hole geometries, they are not suitable for exploring quantum effects, such as Hawking radiation. This is because the model does not lead to the correct commutation relations for the field fluctuations, and so the vacuum state is not well defined.

\subsubsection{Bose-Einstein condensates}

Arguably, the most promising system for implementing analogue models of gravity is the BoseEinstein condensate. This possibility was first considered by Garay et al. [149, 161] for acoustic black hole geometries, and further explored by Barcelo, Visser and Liberati [120,135, 142].

In particular, BECs have a number desirable features with regards to analogue models:

Hydrodynamics: In the long wavelength limit, the equations of motion for a BEC take the form of classical hydrodynamics for a superfluid. In fact, at zero temperature the macroscopic state can be simply described by two degrees of freedom (for each point in space), the density and superfluid velocity (or equivalently, the phase). The resulting description is for a irrotational, inviscid and barotropic fluid. Consequently, the formulation of an emergent spacetime is greatly simplified (see the derivation in section 5.2.1).

Quantum theory: Bose-Einstein condensation in atomic vapours is a weakly interacting system, for which the microscopic quantum theory is well understood. In particular, the elementary excitations of the system are given by considering the linearised fluctuations about the ground state, which is given by a mean-field order parameter. The resulting Bogoliubov theory is accurate near zero temperature. The significance of this result, is that it is valid beyond the hydrodynamic approximation, and so describes the trans-Planckian physics for the effective field theory. Moreover, the weakly interacting regime is amenable to powerful simulation methods for quantum many-body systems, such as the classical field method; this is introduced in chapter 7.

Temperature: Typically, Hawking radiation is a very small effect, and may not be observable in analogues based on classical fluids because of the presence of (larger) thermal fluctuations that obscure the effect. However, the Bose-Einstein condensation in atomic vapours necessarily requires temperatures close to absolute zero, so that the Hawking effect may — in principle — be observable. For example, Barcelo et al. [135] have estimated that it should be possible to achieve a Hawking temperature of $T_{H} \approx 70 \mathrm{n} \mathrm{K}$ in such a system, compared to a critical temperature for Bose-Einstein condensation of $T \approx 90 \mathrm{n} \mathrm{K}$.

Experimental advances: Finally, we note that recent experimental advances for the control of ultra-cold atoms mean that BECs can now be prepared and manipulated in a great many configurations. Notably, the use of magnetic and optical traps can lead to a variety of geometries, whereas with the use of Feshbach resonances it is possible to vary the interaction strength between atoms, even by many orders of magnitude. It is also worth noting that the formation of sonic horizons may be facilitated by the relatively low speed of sound that occurs in BECs. With continued advances the goal of implementing a specific analogue model in the laboratory may be achievable in the near future. 


\subsection{The Hawking Effect}

We introduce the Hawking effect for cosmological black holes, which motivates the development of the analogue models of black holes. We will present a specific model in part III of this thesis.

The most surprising and important prediction to emerge from semi-classical gravity is the discovery that black holes in fact radiate with a temperature that depends on its mass, the original calculation being carried out by Hawking $[162,163]$. The result is particularly significant as it occurs in a regime where both quantum theory and general relativity are prerequisites. (A number of comprehensive reviews on the topic are available elsewhere; see for example [121,164-167].)

Although we do not reproduce the entire calculation here, we outline the salient points with regards to Hawking radiation and its derivation.

- Hawking radiation is the thermal emission of particles from a black hole at a temperature $T_{H}$ given by

$$
k_{B} T_{H}=\frac{\hbar}{2 \pi c} \kappa
$$

where $\kappa$ is the surface gravity at the event horizon for the black hole.

- Hawking's original derivation considered a massless scalar field near a spherically symmetric black hole formed by gravitational collapse, the geometry being described by the Schwarzschild metric. In this case the surface gravity is inversely proportional to the mass of the black hole.

- This calculation involved taking a positive frequency mode at late times, and propagating it backwards in time to before the black hole was formed, the field initially being in a vacuum state for zero scalar particles. The particle production was then calculated by finding the Bogoliubov coefficients between the early (in modes) and late (out modes) times. (A discussion of the Bogoliubov mode mixing formalism is given in section 6.3.)

- There are several equivalent views of Hawking radiation as a physical process. One is pair production due to mixing between positive and negative energy modes at the horizon [163]. Another is quantum mechanical tunneling of particles through the horizon [168].

- The Hawking effect is consistent with thermodynamic considerations; the thermal emission is balanced by a corresponding decrease in the mass of the black hole, so that the process is often termed black hole evaporation. This is formalised by a generalised version of the second law of thermodynamics where a black hole is assigned an entropy - called Bekenstein entropy - this being proportional to its surface area [169].

\section{Minimal conditions for Hawking effect}

Visser has considered the minimum requirements to establish the Hawking effect in black holes $[125,170]$. In particular, he showed the Hawking effect does not depend on a description in terms of either the Einstein equations or Bekenstein entropy - Hawking radiation is a purely kinematic 
effect that should occur in any Lorentzian geometry with an apparent horizon with non-zero surface gravity and sufficiently slow evolution of the geometry. Note in particular, that an event horizon (or absolute horizon) is not required to complete the formulation, removing the usual necessity to connect modes in the infinite future with modes in the infinite past. Thus, this result is particularly relevant for analogue models of gravity, where the concepts of the Einstein equations, Bekenstein entropy and absolute horizons need not necessarily apply.

\subsection{Modifications to the analogy}

\subsubsection{Extensions to theory of Hawking Radiation}

Although the prediction of Hawking radiation is considered to be one of the main accomplishments of quantum field theory in curved spacetime, it relies on a number of assumptions that may turn out to be false, which in turn could invalidate or at least modify the main result. We survey these issues here, although a comprehensive review is provided in [171], and in the context of analogue models in $[117,119]$.

\section{Trans-Planckian problem}

A major limitation of Hawking's original derivation arises due to the inclusion of modes that propagate with a frequency much higher than the Planck frequency. In particular, the out-going modes are exponentially blue-shifted into the trans-Planckian regime for backwards time propagation towards the event horizon. Conventional quantum theory cannot be expected to properly account for these high frequency modes, and thus the standard quantisation of the field may not be appropriate. It seems necessary that a robust theory of Hawking radiation should work for all scales.

Indeed the initial suggestion by Unruh of a fluid analogue for Hawking radiation was motivated by this issue [103], and further analysed by Jacobson [172]. Subsequently, there have been a number of attempts to address the trans-Planckian problem by using a modified dispersion relation for the high frequency modes. In particular, Unruh applied this reasoning to the fluid model [173]. By implementing a modified dispersion relation in addition to a cutoff for the modes, he showed there was no deviation from the usual thermal result for the Hawking effect. Other variations on this approach have also reached similar conclusions [174-179]. More recently, Unruh and Schutzhold have explored modifications to high frequency dispersion where the thermal result is not reproduced [180]. It should be noted that the use of a modified dispersion relation amounts to the formulation of a theory that is not Lorentz invariant.

In general, it is possible to categorise the dispersion of modes in different systems as follows (employing the scheme from [181]):

1. Type I, no dispersion: This behaviour is exhibited by photons in conventional relativity, or by phonons propagating in a fluid in the long wavelength limit.

2. Type II, subluminal dispersion: This behaviour is exhibited by phonons propagating in superfluid Helium for example. We note several studies of Hawking radiation with subliminal dispersion [173-175, 182]. 
3. Type III, Superluminal dispersion: This behaviour is exhibited by phonons propagating in a weakly interacting Bose-Einstein condensate for example. We note the following studies of Hawking radiation with superluminal dispersion [177, 180].

\section{Back-reaction}

Quantum field theory in curved spacetime is usually formulated as a semiclassical theory of gravity: the background spacetime geometry is a classical field upon which quantised fields propagate. Moreover, the standard approach uses a linear field theory that neglects fluctuations in the background - that is, the back-reaction of quantum fields on the metric tensor is ignored. This point of view is perhaps best summed up by Hawking himself, from his original paper on black hole radiation [163]:

"This negative energy flux will cause the area of the event horizon to decrease and so the black hole will not, in fact, be in a stationary state. However, as long as the mass of the black hole is large compared to the Planck mass $10^{-5} \mathrm{~g}$, the rate of evolution of the black hole will be very slow compared to the characteristic time for light to cross the Schwarzchild radius. Thus it is a reasonable approximation to describe the black hole by a sequence of stationary solutions and to calculate the rate of particle emission in each solution. Eventually, when the mass of the black hole is reduced to $10^{-5} \mathrm{~g}$, the quasi-stationary approximation will break down."

Evidently Hawking radiation is considered to be a kinematic effect; that is, when back-reaction is neglected it does not depend on the overall dynamical evolution of the system. However, it is still unclear what effect this approximation has on Hawking radiation. As a first step, it is possible to incorporate back-reaction into the semi-classical framework by using a dynamical field equation. This is equivalent to replacing the energy-momentum tensor $T_{\mu \nu}$ with its quantum expectation value in Einstein's equations, and solving this self-consistently [183, 184]. Parikh and Wilzcek [168] employed this approach by allowing the black hole mass to vary so that energy conservation was enforced, resulting in higher order corrections to the usual thermal spectrum. A full treatment of this problem would require a theory of quantum gravity, whereby spacetime itself would be quantised and therefore on an equal footing with matter fields.

The problem of back-reaction is particularly relevant in analogue models of gravity. This is the case for the following reasons:

- The emergent spacetime geometry and quantum fluctuations are both described by the same underlying theory for the constituent matter - for example, for Bose-Einstein condensates this would be the quantum theory of the atomic gas. Therefore in principle there should be no conceptual difficulty with incorporating the back-reaction of the quantum field on the effective metric.

- An experiment for analogue gravity in the laboratory constitutes a closed system, and so the effects of any quantum fluctuations on the background should be necessarily included. 
- Although the usual semi-classical treatment considers a quantum field coupled to a classical background, it may not be possible to carry over this line of reasoning to quantum fluids, such as Bose-Einstein condensates. In this case, there may be additional conservation requirements and some transitions may not be permitted. In particular, in a toroidal geometry, there is a topological constraint of quantised circulation.

Despite these points, back-reaction is often neglected in analogue models of gravity; indeed this point of view is valid as long as the background geometry is amenable to a mean field description. This amounts to a linear field theory where nonlinear fluctuation terms are neglected, so that there are no interactions between the background and quasiparticle modes of the system.

There have been various attempts to incorporate back-reaction effects into analogue models of gravity. Balbinot et al. $[119,185]$ have studied the effects of back-reaction in a sonic horizon formed by a nozzle. They found a correction to the emission temperature, which decreases as time evolves. This is in direct contrast to the situation in (cosmological) Schwarzschild black holes where the temperature increases as the black hole evaporates. Schutzhold et al. [186] found the semi-classical approach failed to account properly for effects of back-reaction in Bose-Einstein condensates. Another approach is stochastic gravity whereby a stochastic term is included in Einstein's equations, which accounts for quantum fluctuations of the energy-momentum tensor [187].

A closely related issue to back-reaction is that of the stability of the horizon itself. This has been investigated by Liberati et al. [188] who showed that for the case of a classical fluid there is a regularity condition required so that the surface gravity does not diverge at the horizon.

\section{Interacting fields}

Finally, it is worth mentioning that in general, quantum field theory in curved spacetime involves free fields - that is, fields that do not interact. The extension of the theory to self-interacting fields can be realised by the inclusion of a nonlinear term in the Lagrangian density, which leads to the so-called $\phi^{4}$ theory [121].

\subsubsection{Relevance to analogue models in BECs}

In light of these issues, analogue models of gravity may provide an interesting avenue for observing the analogue of Hawking effect and therefore for investigating the validity of the original arguments in its derivation. This is further reinforced by the fact that cosmological black holes are inaccessible to observation, so that the experimental confirmation of the Hawking effect has not yet been achieved. Moreover, while much of the above discussion has focused on the Hawking effect we can also expect modifications to inflationary cosmology models — and their analogues — due to some or all of the above issues.

It is worth re-iterating the assumptions in making the case for analogue gravity models in Bose-Einstein condensates. The theory resulting from linearised quantum fluctuations leads to a free-field theory — that is, the modes of the field are non-interacting. Moreover, the backreaction of the quantum fluctuations on the condensate mode is neglected; this is equivalent to assuming 
the metric tensor is a classical quantity. Finally, the analogy is rigorous only within the acoustic approximation - ie. for low momenta modes.

The classical field method that will be introduced in chapter 7 does not require these assumptions; here all modes of the system are included in the field evolution, and these modes are able to interact via the nonlinear interaction term. Thus the classical field method allows us to explore the validity of the assumptions of the free-field theory. Moreover, in a Bose-Einstein condensate, short wavelength modes (of the order of less than the healing length) exhibit high-frequency dispersion and therefore play the role of super-Planckian modes in QFTCS - this generally leads to a theory that violates Lorentz invariance. The classical field method naturally incorporates these effects since non-phononic modes are also represented by the field.

\subsection{Motivation and outline}

In the remainder of this thesis, we explore two different analogue models of gravity in BoseEinstein condensates. In particular: in part II we investigate an analogue model of a flat Friedmann-Robertson-Walker universe undergoing an expansion; in part III we investigate a configuration for an acoustic black hole formed by two de Laval nozzles in a ring, which exhibits both black and white hole horizons. The motivation for these studies is to extend the typical analyses, which are made in the acoustic approximation, by utilising the full (effective) quantum field theory for BECs. To this end we make use of the linearised theory of excitations - known as Bogoliubov theory - to characterise the quasiparticle excitations of the system, and apply the classical field method for field dynamics. We expect therefore to model what would actually be observed for a BEC in the experimental configurations required to implement either of the proposed analogue models of gravity. It should be emphasised that while our results have an interpretation in terms of analogue models, the formalism does not depend on such an interpretation. 


\title{
Chapter 6
}

\section{Expanding universe models in Bose-Einstein condensates}

\author{
Anyone informed that the universe is expanding and contracting in pulsations of \\ eighty billion years has a right to ask, "What's in it for me?" \\ Peter De Vries
}

\subsection{Introduction}

In the theory of quantum fields in classical backgrounds, some form of particle creation is expected when the metric is time-dependent; a commonly cited example of this is cosmological particle production in an expanding universe [121, 189]. It is possible to simulate the analogy of this process in a Bose-Einstein condensate (BEC) when either: the external trapping frequency is time-dependent [104-108]; or the scattering length (within the low momentum approximation of the two-body interaction potential) is time-dependent [109-111]. These treatments are often based on the acoustic (ie. hydrodynamic) approximation where it is assumed that all excitations of the quantum field propagate as phonons with the same speed of sound. Moreover back-reaction of the excitations on the background field and higher order interactions between excitations are neglected in the linearised theory.

In general, the correct description of the dynamics of a BEC is a formidable problem due to the vastness of the Hilbert space even for a system of just a few interacting atoms. In the lowest order approximation, when all the bosonic atoms occupy a single quantum state, the ground state is well described by the Gross-Pitaevskii equation (GPE) - in this case, the field operator is replaced by a mean-field order parameter. Classical field methods (CFM) extend this formalism to include quantum fluctuations whereby the dynamics of a multimode quantum field is approximated by the trajectories of classical variables in phase space. One such method is the truncated Wigner approximation (TWA), which is based on the Wigner representation of the density matrix. The TWA has been investigated by a number of authors [34-37] and more recently has been applied to a study of condensate collisions $[41,42]$.

In this part of the thesis we investigate the dynamics of a homogeneous BEC in two spatial dimensions with a time dependent scattering length, and in particular, map this problem to a 
Friedmann-Robertson-Walker (FRW) universe undergoing an expansion. We compare the calculated particle production from the acoustic approximation with the results of numerical simulations based on the TWA. There are several benefits to this approach. Firstly, the TWA includes the effects of vacuum quantum fluctuations by sampling the Wigner distribution in the initial state. Secondly, the field dynamics naturally include the nonlinear dispersion of the Bose system - in a cosmological context, this represents Lorentz symmetry breaking of the effective spacetime which leads to necessary modifications of the standard hydrodynamic theory. Finally, the numerical simulations include the effects of back-reaction which is difficult to otherwise include without resorting to higher order methods such as the self-consistent Hartree-Fock-Bogoliubov approach.

The outline of this part of the thesis is as follows: In this chapter we show how the acoustic metric leads to an effective FRW universe and give results for particle production for three different expansion scenarios; Chapter 7 introduces the TWA which we use to simulate the dynamics of the Bose-Einstein condensate consistently with these scenarios; Chapter 8 compares the results of the TWA with the model predictions, concludes and discusses avenues for further work.

\subsection{Emergent FRW Universe}

The analogy between the hydrodynamic description of a Bose-Einstein condensate and curved spacetime was established in chapter 5. Although the metric tensor (5.24) includes a conformal factor, this does not affect the dynamics of the field (in this case the mode functions need to be renormalised for consistency with the Bogoliubov theory). Time dependence can enter in any of the parameters $n_{0}, c$ and $\mathbf{v}$. We focus on the case where the background flow is zero $(\mathbf{v}=0)$, and the system is homogeneous with a density $n_{0}$ that is constant through space. With this choice of parameters the effective metric (5.24) becomes

$$
g_{\mu \nu}=\left(\frac{n_{0}}{c}\right)^{\frac{2}{d-1}}\left[\begin{array}{ccc}
-c^{2} & \vdots & 0 \\
\ldots & & \ldots \\
0 & \vdots & \delta_{i j}
\end{array}\right]
$$

This represents a Friedmann-Robertson-Walker spacetime [109,110], and corresponds to the notion of an effective expansion that we introduced in section 5.3.1. Such geometries are conformally flat and at any particular time the spatial geometry is simply that of Euclidean flat space. The time dependence is contained entirely in the speed of sound given by

$$
c(t)^{2}=\frac{U(t) n}{m}=\frac{4 \pi \hbar^{2}}{m^{2}} n a(t),
$$

with atoms of mass $m$, scattering length $a$ and number density $n$; we introduce the dimensionless scaling function $b(t)$ so that the interaction strength (or equivalently the scattering length) has the time dependence

$$
U(t) \equiv U_{0} b(t),
$$


where $U_{0}=U\left(t_{0}\right)$ at an initial time $t_{0}$; therefore we take $b\left(t_{0}\right)=1$. From (6.2) the time dependence of the speed of sound is thus

$$
c(t)=c_{0} b(t)^{1 / 2}
$$

In practice a variation in the interaction strength is possible by using a Feshbach resonance $[137,138]$. If $b(t)$ is decreasing with time we have an expanding universe model, whereas if $b(t)$ increases with time we have a contracting universe model.

The line element for the FRW universe we have described is given by

$$
d s_{\text {eff }}^{2}=g_{\mu \nu} d x^{\mu} d x^{\nu}=\Omega_{0}^{2}\left[-c_{0}^{2} b(t)^{\alpha} \mathrm{d} t^{2}+b(t)^{\alpha-1} \mathrm{~d} \mathbf{x}^{2}\right]
$$

We have introduced the conformal factor

$$
\Omega_{0}^{2}\left(n_{0}, c_{0}, d\right)=\left(\frac{n_{0}}{c_{0}}\right)^{\frac{2}{d-1}}
$$

which is independent of space and time, as well as the dimension-dependent exponent

$$
\alpha=\frac{d-2}{d-1}
$$

In what respect do we have an expanding universe, given that the condensate is contained in a fixed volume $V$ ? A decrease in the scattering length corresponds to a decrease in the speed of sound propagating in the condensate; therefore any acoustic excitations will propagate with decreasing speed in the condensate as time passes. To an observer at rest in the effective spacetime, a decrease of the speed of sound is thus indistinguishable to an isotropic expansion of the spatial dimensions.

It is not straightforward to define either an apparent or event horizon in the model considered here as the system is homogeneous, and the background velocity is therefore the same (ie. zero) everywhere. This is further complicated by the fact that the causal structure of the effective spacetime should be determined by the maximum signal velocity (ie. group velocity), which is effectively infinite here owing the superluminal modes in a BEC. Analogue models that certainly do have horizons exist for systems where the background velocity depends on the radial position - for example, when the trapping potential is switched off and the condensate is free to expand [104-108]. In the present situation, where the scale factor $a_{\mathrm{FRW}}(t)$ or equivalently $b(t)$ contains all the geometric structure for the spacetime, it is necessary to perform the usual analysis in terms of cosmological horizons to determine the overall causal structure of the spacetime [190].

\subsubsection{Two dimensional model}

To facilitate the numerical calculations required by the classical field simulations that we present in chapter 8 , we continue with $d=2$ spatial dimensions. The reduced mode space for $d=2$ greatly decreases the computation time, but still leads to a satisfactory description of the system. In particular, we expect the extension of the numerical simulations to $d=3$ to lead to qualitatively 
similar results ${ }^{1}$. Moreover, while it has been shown $[107,108]$ that for $d=2$, a condensate undergoing free expansion leads to a scalar field equation that does not depend on the scaling factor in co-moving coordinates ${ }^{2}$ - so that in this case there can be no particle production as we will see, the present model for a $2+1$ dimensional FRW universe leads to a scalar field equation that does allow particle production.

For $2+1$ spacetime dimensions, $\alpha(2)=0$ and the line-element simplifies to

$$
d s_{\text {eff }}^{2}=\left(\frac{n_{0}}{c_{0}}\right)^{2}\left[-c_{0}^{2} \mathrm{~d} t^{2}+b(t)^{-1} \mathrm{~d} \mathbf{x}^{2}\right]
$$

A further time transformation is not required as laboratory time and proper time (for a comoving observer) are of the same form. The scaling factor $a_{\mathrm{FRW}}(t)$ for a FRW universe that is familiar from cosmology (see equation 5.28) is related to $b(t)$ by

$$
a_{\mathrm{FRW}}(t)=b(t)^{-1 / 2}
$$

for $d=2$. (We always explicitly specify the FRW subscript for the cosmological scale factor so that this quantity is not confused with the s-wave scattering length.)

The reduction of the model to two dimensions requires a modification to the nonlinearity that appears in the GPE (5.1), and therefore also the resulting field equation (5.14). To see this, we assume the transverse $z$ dimension is tightly confined with the trap lengths satisfying $L_{z} \ll L_{x}$, $L_{y}$, and further that the $L_{z} \sim \xi$ for the transverse dimension where $\xi$ is the healing length of the condensate. The scattering is still determined by the three-dimensional scattering length so that this is called a quasi-two dimensional geometry. However, the system remains in the ground state of the transverse dimension because the energy required for transverse excitations is much larger than for longitudinal excitations. The wavefunction is then separable as $\Psi(\mathbf{x}, t)=\psi(x, y, t) \zeta(z)$. Assuming the condensate is homogeneous the GPE can be rewritten as

$$
i \hbar \frac{\partial \psi(x, y, t)}{\partial t}=\left(-\frac{\hbar^{2}}{2 m} \nabla^{2}+V_{\text {ext }}\right) \psi(x, y, t)+U_{2 \mathrm{D}}|\psi(x, y, t)|^{2} \psi(x, y, t)
$$

where the effective nonlinearity is $U_{2 \mathrm{D}}=U / L_{z}$. This does not affect the form of the resulting calculations for particle production, but should be noted when we determine suitable parameters for our simulations in section 7.4.

\footnotetext{
${ }^{1}$ This is because the projected Gross-Pitaevskii equation, which the classical field method is based on, takes the same form for $d=2,3$. While the extension of the classical field method to $d=3$ spatial dimensions is straightforward, in order to have investigated the range of different expansion scenarios we have considered in this thesis, would have required a unfeasibly large computational time on one or a few workstations.

${ }^{2}$ The models considered in $[107,108]$ correspond to the case of a physical expansion as introduced in section 5.3.1. The scaling factor there has a different physical significance from the scaling factor for the effective expansion that is considered here.
} 


\subsection{Field quantisation and particle production}

An arbitrary field $\hat{\theta}(t)$ can be quantised using the plane wave mode expansion:

$$
\hat{\theta}(\mathbf{x}, t)=\frac{1}{\sqrt{V}} \sum_{\mathbf{k}}\left[\hat{b}_{\mathbf{k}} e^{i \mathbf{k} \cdot \mathbf{x}} \chi_{\mathbf{k}}(t)+\hat{b}_{\mathbf{k}}^{\dagger} e^{-i \mathbf{k} \cdot \mathbf{x}} \chi_{\mathbf{k}}^{*}(t)\right]
$$

where $\hat{b}_{\mathbf{k}}$ and $\hat{b}_{\mathbf{k}}^{\dagger}$ are the annihilation and creation operators respectively for the quasiparticle modes.

In flat (Minkowski) spacetime we can associate the positive and negative frequency solutions of (5.17) with annihilation and creation operators respectively. In curved spacetime, this association is not always possible as the mode expansion need no longer be unique; in the language of general relativity, the spacetime does not have a (globally) time-like Killing vector field so that the positive frequency solution is not necessarily an eigenfunction of $\partial_{t}$. A consequence of this is that the choice of a Fock vacuum according to $\hat{b}|0\rangle=0$ depends in general on the choice of coordinates; that is, the measurement of particle content in curved spacetime is said to be "observer dependent".

The calculation of particle production follows the standard methodology [121,189]. We define in and out regions respectively as asymptotically flat regions with $t \rightarrow-\infty$ and $t \rightarrow+\infty$. (While the existence of asymptotically flat regions cannot be assumed for cosmological models, it should certainly be possible to emulate this scenario in BEC experiments.) We can write a mode expansion (6.11) for the in and out regions in terms of mode functions $\chi_{\mathbf{k}}^{\text {in }}$ or $\chi_{\mathbf{k}}^{\text {out }}$ respectively. Because both sets of modes are a basis set for the field, they are related by the Bogoliubov transformation

$$
\chi_{\mathbf{k}}^{\text {out }}=\alpha_{\mathbf{k}} \chi_{\mathbf{k}}^{\text {in }}+\beta_{-\mathbf{k}} \chi_{-\mathbf{k}}^{\text {in } *}
$$

Note the spacetime has translational invariance as a symmetry so the field (6.11) can be expanded in the same set of plane wave modes $e^{i \mathbf{k} \cdot \mathbf{x}}$ for both the in and out regions, and therefore $\beta_{-\mathbf{k}}=\beta_{\mathbf{k}}$ also. By convention the mode functions are normalised according to the Klein-Gordon inner product. In momentum space this takes the form

$$
-i W\left[\chi_{\mathbf{k}}, \chi_{\mathbf{k}}^{*}\right]=1
$$

where $W\left[f_{1}, f_{2}\right]=f_{1}\left(\partial_{t} f_{2}\right)-\left(\partial_{t} f_{1}\right) f_{2}$ is the Wronskian of two functions $f_{1}$ and $f_{2}$.

For each asymptotic region the vacuum state is defined by the requirement

$$
\begin{aligned}
b_{\mathbf{k}}^{\text {in }}\left|0^{\text {in }}\right\rangle & =0 \\
b_{\mathbf{k}}^{\text {out }}\left|0^{\text {out }}\right\rangle & =0
\end{aligned}
$$

The in and out mode operators are also related by

$$
b_{\mathbf{k}}^{\text {out }}=\alpha_{\mathbf{k}} b_{\mathbf{k}}^{\text {in }}+\beta_{-\mathbf{k}} b_{-\mathbf{k}}^{\text {tin }}
$$


The number of particles created is then given by the quantity

$$
\begin{aligned}
N_{\mathbf{k}}^{\text {out }} & =\left\langle 0^{\text {in }}\left|b_{\mathbf{k}}^{\text {†out }} b_{\mathbf{k}}^{\text {out }}\right| 0^{\text {in }}\right\rangle \\
& =\left|\beta_{\mathbf{k}}\right|^{2}
\end{aligned}
$$

The standard procedure to calculate $\beta_{\mathbf{k}}$ involves solving the field equation (5.14) for the in and out regions by using (6.12) with the appropriate boundary conditions. In the acoustic approximation, the form of the field equation (5.17) means that this procedure is mathematically equivalent ${ }^{3}$ to solving the one dimensional Schrodinger equation for above-barrier scattering from a potential [191].

It should be stressed that here particle production refers to the production of (massless) quasiparticle excitations in the Bogoliubov basis, that approximately diagonalises the many body Hamiltonian for the Bose gas (see section 1.2.1). As (6.15) couples modes of momenta $\mathbf{k}$ and $-\mathbf{k}$ this process is often referred to as pair production and is associated with the formation of squeezed states $[166,192]$.

\section{Choosing a Fock vacuum}

When the in and out regions of the expansion are not asymptotically flat, a vacuum state cannot be unambiguously defined for either case. However, clearly the procedure to calculate particle production outlined above requires a choice of Fock vacuum, and moreover, that choice should lead to physically reasonable results. We therefore mention two choices that have been developed to deal with this situation (although there are many more):

The instantaneous Minkowski vacuum: this is the state that corresponds to the instantaneous diagonalisation of the Hamiltonian at a given time (and is therefore also the state that minimises the energy). This choice may be problematic in some cases as it can lead to situations of infinite particle production even though the expansion may be smooth and finite [189].

The adiabatic vacuum: this approximation can be used for modes that experience a sufficiently slow expansion and is formulated in terms of the WKB approximation [121,193]. However, the adiabaticity requirement means it has limited applicability.

In section 6.5.3 we calculate particle production for the case of a de Sitter expansion for which there are no static regions - in this case we therefore utilise the instantaneous Minkowski vacuum to calculate the particle production at a given time during the expansion. The problem of infinite particle production does not occur in this case, and as we shall see from the classical field simulation results in chapter 8, particle production is further suppressed for short wavelength modes because of superluminal dispersion.

\footnotetext{
${ }^{3}$ The correspondence follows by exchanging the time and spatial coordinates in the field equation.
} 


\section{Commutation relations}

In addition to identifying an effective metric — and therefore also a field equation - required to make the analogy with QFTCS, another prerequisite is that in quantising the field the operators should be annihilation and creation operators in the usual sense. That is, they should obey the correct commutation relations so that we can define quanta of the field in the Fock basis. This has indeed shown to be the case for the linear excitations of a BEC [160], and also follows from the discussion in section 6.4.1

\section{Relevant scales}

There are two relevant scales for particle production in a FRW-type analogue model:

1. Hubble parameter - Following [109] for the metric (6.8) the Hubble parameter is given in terms of the scaling function $b(t)$ in laboratory time by

$$
H \equiv \frac{\dot{a}_{\mathrm{FRW}}}{a_{\mathrm{FRW}}}=-\frac{1}{2} \frac{\dot{b}}{b}
$$

This quantity, which is time-dependent in general, corresponds to the rate of expansion for the universe. If $H \gtrsim \omega_{k}$ for a mode frequency labeled by $k$ the dynamics are Hubbledominated and we expect the mode to be to be non-oscillating, whereas $H \ll \omega_{k}$ implies the mode is oscillating and in the adiabatic regime. In the latter case this is what cosmologists refer to as a parametrically excited mode. Clearly a large value for $H$ is favourable to particle production - however $H$ can not be made arbitrarily large as the approximations that lead to the effective field theory would then be violated $[109,194]$

2. Healing length - In a Bose-Einstein condensate, the healing length is a measure of the smallest distance that supports density fluctuations (ie. when a vortex core forms, the healing length is comparable to the radius of the vortex core), and is given by

$$
\xi=\frac{\hbar}{\sqrt{2} m c}=\xi_{0} b(t)^{-1 / 2}
$$

That is, if we define the cross-over from phonon to free-particle behaviour for the Bogoliubov spectrum (6.46) as $\hbar^{2} k_{c}^{2} / 2 m \equiv U n$ then $k_{c}=1 / \xi$. Modes for which $k \ll 1 / \xi$ correspond to collective excitations of the condensate (phonons) and couple to the effective time-dependent curved spacetime whereas modes with $k \gg 1 / \xi$ are particle-like and are relatively unaffected by the effective spacetime geometry. Alternatively stated, the spacetime appears locally flat and time independent to modes with short wavelengths.

Particle production into a mode $\mathbf{k}$ then proceeds as follows: during an expansion, while $H \gtrsim$ $\omega_{k}$ and $k<1 / \xi$, the mode evolves non-trivially and particle production will certainly occur. As the expansion proceeds, particle production can "switch off" for two reasons: Either the healing length increases until the mode becomes particle-like and particle production slows, ceasing altogether when $k \gg 1 / \xi$; alternatively, if the expansion slows, then after some time $H \lesssim \omega_{k}$ so that the 
field begins to oscillate relatively freely, and additional particle production also ceases. In either case, the mode no longer evolves, and the occupation number of the mode becomes constant.

In general, it can be difficult or impossible to analytically solve the field equation (5.14) for the mode functions. Moreover, if the in region is not flat, there is no preferred choice for the initial vacuum state. However, when the field evolution is sufficiently slow - ie. adiabatic the WKB approximation can be used to calculate particle production (see [193] and references therein). It is worth noting that this approach leads to a Planckian spectrum in the lowest order approximation [121].

\section{A note on freezing}

For our analogue model of an expanding FRW universe, in the acoustic approximation we have $\omega_{k}(t)=c(t) k$, which decreases as the expansion proceeds (this is equivalent to the usual notion of cosmological redshifting of modes that occurs during inflation). This means that depending on the form of the expansion, a mode that is initially oscillating $\left(H \ll \omega_{k}\right)$ may enter a Hubbledominated era $\left(H \gg \omega_{k}\right)$ after a sufficiently long expansion - this is the mechanism for freezing of modes that is familiar from inflationary cosmology. The term freezing does not necessarily imply that particle production ceases, but rather, that the mode no longer oscillates.

The situation is quite different when nonlinear superluminal dispersion is included - in this case, the healing length $\xi$ also decreases for increasing time, which means that a mode that was initially phononic will crossover into the adiabatic free-particle regime for a sufficiently long expansion. In this case therefore, the notion of freezing can only occur in a transient regime that depends on the mode wave-vector $k$ and the form of the scaling function $b(t)$ (and therefore on the Hubble parameter also).

\subsubsection{Acoustic approximation}

When the quantum pressure term is neglected in the equations of motion (5.9) and (5.10), the correspondence between the massless and minimally coupled scalar field equation for a FRW universe and the equations of motion for linearised fluctuations in the BEC is exact. The dynamics of the low momentum modes are expected to fall into this regime, and therefore it is of interest to examine this case first.

\section{Expansion}

It is useful to introduce $X$ the expansion of the universe between two times $t_{0}$ and $t_{f}$ in the following way:

$$
X=\frac{U\left(t_{0}\right)}{U\left(t_{f}\right)}=\frac{1}{b\left(t_{f}\right)}
$$

\section{Conformal time}

We define a coordinate transformation from laboratory time $t$ to conformal time $\eta$ by

$$
\mathrm{d} \eta=\sqrt{b(t)} \mathrm{d} t
$$


The line element (6.8) then reads

$$
d l_{\text {eff }}^{2}=\Omega_{0}^{2} b(\eta)^{-1}\left[-c_{0}^{2} \mathrm{~d} \eta^{2}+\mathrm{d} \mathbf{x}^{2}\right],
$$

The equation of motion for the field obtained from (5.14) in conformal time becomes

$$
\partial_{\eta}^{2} \hat{\theta}-\frac{1}{2} \frac{\dot{b}(\eta)}{b(\eta)} \partial_{\eta} \hat{\theta}-c_{0}^{2} \nabla^{2} \hat{\theta}=0
$$

Note that the coefficient of $\partial_{\eta} \hat{\theta}$ is the Hubble parameter given by (6.17). The calculation of particle production requires the solution of this field equation for the mode functions $\chi(\eta)$. This task is assisted by reducing the field equation to standard form where the first order derivative does not appear; this can be achieved (for instance) by introducing an auxiliary field of the form $\hat{\varsigma}(\eta)=b(\eta) \hat{\theta}$, showing mathematically equivalent dynamics to $\hat{\theta}$. Alternatively we can consider the transformation to auxiliary time as follows.

\section{Auxiliary time}

Defining the auxiliary factor by

$$
\Lambda=\Omega_{0}^{2 / 3} b(t)^{-1 / 3}
$$

and the auxiliary time by

$$
\tilde{t}=\int \frac{c_{0} b(t)}{\Omega_{0}} d t
$$

the line element (6.8) transforms as

$$
d l_{\mathrm{eff}}^{2}=-\Lambda^{6} d \tilde{t}^{2}+\Lambda^{3} d \mathbf{x}^{2}
$$

For a massless scalar field, the corresponding field equation is

$$
\partial_{\tilde{t}}^{2} \hat{\theta}-\Lambda^{3} \nabla^{2} \hat{\theta}=0
$$

Using the field mode expansion given by (6.11) we then get a time-dependent harmonic oscillator for each mode

$$
\partial_{\tilde{t}}^{2} \chi_{\mathbf{k}}+\tilde{\omega}_{\mathbf{k}}^{2}(\tilde{t}) \chi_{\mathbf{k}}=0
$$

where $\tilde{\omega}_{\mathbf{k}}^{2}(\tilde{t})=\Lambda^{3} k^{2}=\Omega_{0}^{2} b(\tilde{t})^{-1} k^{2}$ is the oscillator frequency. While the auxiliary time approach is useful in the acoustic approximation, it does not generally lead to the simple form (6.27) when the quantum pressure term is included (see section 6.3.2). 


\subsubsection{Beyond the acoustic approximation}

The results of the previous section were given within the acoustic approximation, valid only for long wavelength modes, whereby the quantum pressure term is omitted from the field equation (5.14) and the resulting equations for the FRW analogue model given by (6.22) or (6.27). We can extend the analysis to higher momenta modes by including the quantum pressure term; this leads to a "superluminal" type dispersion relation for high frequency modes and an appropriately modified field equation as we presently discuss. Moreover, this description corresponds to the presence of "trans-Planckian" like modes, and leads to the idea of Lorentz violation. Such modified dispersion relations have similarly been incorporated into some studies of inflationary cosmology - we discuss this here also. In section 6.6 we will consider two specific forms of the scaling function in this regime: the limiting case of a sudden transition (section 6.6.1) and a cyclic universe model (section 6.6.2)

\section{Nonlinear dispersion}

For the homogeneous model presented here the differential term (5.11), accounting for the quantum pressure term, takes the simple form

$$
\widetilde{D}_{2} n_{1}=\frac{1}{2 n_{0}} \nabla^{2} n_{1}
$$

That is, we can extract the Fourier component

$$
\left(\widetilde{D}_{2} n_{1}\right)_{\mathbf{k}}=-\frac{1}{2 n_{0}} k^{2} n_{\mathbf{k}}
$$

and thus rearrange (5.10) to give

$$
n_{\mathbf{k}}=-\frac{\hbar}{U}\left[\frac{\epsilon_{k}^{0}}{2 U n_{0}}+1\right]^{-1} \partial_{t} \chi_{\mathbf{k}}
$$

where $\epsilon_{k}^{0}=\hbar^{2} k^{2} / 2 m$ is the single-particle energy for a non-interacting gas.

We can then include the quantum pressure term by using (6.30) so that the field equation (5.14) in momentum space becomes

$$
\partial_{t}^{2} \chi_{k}-\frac{1}{2} \frac{c_{0}^{2} k^{2}}{\omega_{k}(t)^{2}} \partial_{t} b(t) \partial_{t} \chi_{k}+\omega_{k}(t)^{2} \chi_{k}=0
$$

where we have defined

$$
\omega_{k}(t)^{2}=\frac{k^{2}}{2 m}\left(\frac{\hbar^{2} k^{2}}{2 m}+2 U(t) n_{0}\right)
$$

It should come as no surprise that we have recovered the Bogoliubov dispersion relation for a weakly interacting Bose gas (compare $\epsilon_{k}=\hbar \omega_{k}$ with equation 6.46). In particular, we see that if we set $b(t)=$ const. then the field equation describes the dynamics of each Bogoliubov mode for a time-independent Hamiltonian. 
In general, it is difficult to solve (6.31) for the mode functions, and to hence calculate particle production. This situation often persists even though it may be possible to reduce (6.31) to standard form where the first order derivative term $\partial_{t} \chi_{k}$ does not appear. We can, however, make a qualitative statement about particle production for high momenta modes by considering the crossover from phononic to free-particle modes as determined by the healing length (6.18). For modes that satisfy $k \gg k_{c}$, the field equation takes the approximate form

$$
\partial_{t}^{2} \chi_{k}+\left(\frac{\epsilon_{k}^{0}}{\hbar}\right)^{2} \chi_{k}=0
$$

That is, the time dependence from $b(t)$ above is largely suppressed, and each mode with $k \gg k_{c}$ evolves trivially as a time-independent harmonic oscillator, remaining in its' initial vacuum state. Therefore these modes experience no particle production.

\section{Lorentz violation}

Planck-scale Lorentz violation is a feature of some theories of quantum gravity that can be modeled by the presence of a modified dispersion relation at the Planck scale [195]. In this sense, for a BEC the healing length $\xi$ provides the analogue of the Planck scale, characterising the cross-over from phononic $(k \ll 1 / \xi)$ to free-particle $(k \gg 1 / \xi)$ like modes. Specifically, the field equation (6.31) is not Lorentz invariant because of the nonlinear superluminal dispersion of the modes (6.32) at large momenta (this clearly evident from equation 6.33 where the second term varies as $\sim k^{4}$ ). On the other hand, for small momenta (ie. in the acoustic approximation) the dispersion relation is linear in $k$ and the field equation reduces to the Lorentz invariant form (6.22). It should be stressed however, that the effective quantum field theory for the BEC is still valid as long as atomic interactions can be characterised by the s-wave scattering length, which is true in general for some cut-off in wave-vector $k_{\text {cut-off }}>1 / \xi$.

We note that modified dispersion relations have been used to study the "trans-Planckian" problem in inflationary cosmology [196] — the results there showed that certain modifications to the dispersion relation could lead to significant deviations for the spectrum when compared to the unmodified dispersion relation (ie. the usual model of inflation).

\subsection{Connection with Bogoliubov Theory}

Thus far, we have derived a field equation for a scalar field propagating in an effective spacetime. The quanta of this scalar field must correspond to the linearised quantised excitations (ie. phonons) of the quantum field for the theory to be consistent. Therefore, at this point, to make this connection explicit, it is worth pursuing the theory of quantum excitations in BECs, which is well described by Bogoliubov's theory of excitations for a weakly interacting system. 


\subsubsection{Bogoliubov Theory}

To first order we can include quantum fluctuations by using Bogoliubov's theory for a homogeneous dilute Bose gas, and thus calculate analytically the excitation spectrum [28, 197]. We proceed by expanding the field operator in a plane-wave basis

$$
\hat{\psi}(\mathbf{x}, t)=\frac{1}{\sqrt{V}} \sum_{\mathbf{k}} e^{i \mathbf{k} \cdot \mathbf{x}} \hat{a}_{\mathbf{k}}(t)
$$

and substituting this in the (1.1). The resulting effective Hamiltonian is then

$$
\begin{aligned}
\hat{H} & =\hat{H}_{0}+\hat{H}_{\mathrm{int}} \\
\hat{H}_{0} & =\sum_{\mathbf{k}} \epsilon_{\mathbf{k}}^{0} \hat{a}_{\mathbf{k}}^{\dagger} \hat{a}_{\mathbf{k}} \\
\hat{H}_{\text {int }} & =\frac{U_{0}}{2 V} \sum_{\mathbf{k} \mathbf{k}^{\prime} \mathbf{q}} \hat{a}_{\mathbf{k}+\mathbf{q}}^{\dagger} \hat{a}_{\mathbf{k}^{\prime}-\mathbf{q}}^{\dagger} \hat{a}_{\mathbf{k}} \hat{a}_{\mathbf{k}^{\prime}}
\end{aligned}
$$

When the number of atoms in the condensate is large (ie. the field is highly condensed) we can make the replacements $\hat{a}_{0}, \hat{a}_{0}^{\dagger} \rightarrow \sqrt{N_{0}}$ and the interaction part of (6.35) becomes

$$
\hat{H}_{\text {int }}=\frac{U_{0}}{2 V}\left[N_{0}^{2}+2 N_{0} \sum_{\mathbf{k} \neq 0}\left(\hat{a}_{\mathbf{k}}^{\dagger} \hat{a}_{\mathbf{k}}+\hat{a}_{-\mathbf{k}}^{\dagger} \hat{a}_{-\mathbf{k}}\right)+N_{0} \sum_{\mathbf{k} \neq 0}\left(\hat{a}_{\mathbf{k}}^{\dagger} \hat{a}_{-\mathbf{k}}^{\dagger}+\hat{a}_{-\mathbf{k}} \hat{a}_{\mathbf{k}}\right)\right]
$$

where only terms of at least order $N_{0}$ have been retained. We can write the total number operator as

$$
\hat{N}=\sum_{\mathbf{k}} \hat{a}_{\mathbf{k}}^{\dagger} \hat{a}_{\mathbf{k}}=N_{0}+\sum_{\mathbf{k} \neq 0}\left(\hat{a}_{\mathbf{k}}^{\dagger} \hat{a}_{\mathbf{k}}+\hat{a}_{-\mathbf{k}}^{\dagger} \hat{a}_{-\mathbf{k}}\right)
$$

Setting $N=\langle\hat{N}\rangle$ we can eliminate $N_{0}$ to get (again retaining only terms at least order $N_{0}$ )

$$
\begin{aligned}
\hat{H} \approx \hat{H}_{\text {Bog }}=\frac{U_{0} N^{2}}{2 V}+\frac{1}{2} \sum_{\mathbf{k} \neq 0}[ & \left(\epsilon_{\mathbf{k}}^{0}+\frac{U_{0} N}{V}\right)\left(\hat{a}_{\mathbf{k}}^{\dagger} \hat{a}_{\mathbf{k}}+\hat{a}_{-\mathbf{k}}^{\dagger} \hat{a}_{-\mathbf{k}}\right) \\
& \left.+\frac{U_{0} N}{V}\left(\hat{a}_{\mathbf{k}}^{\dagger} \hat{a}_{-\mathbf{k}}^{\dagger}+\hat{a}_{-\mathbf{k}} \hat{a}_{\mathbf{k}}\right)\right]
\end{aligned}
$$

This can be diagonalised using the Bogoliubov transformation

$$
\begin{aligned}
\hat{a}_{\mathbf{k}} & =u_{\mathbf{k}} \hat{b}_{\mathbf{k}}+v_{\mathbf{k}} \hat{b}_{-\mathbf{k}}^{\dagger} \\
\hat{a}_{-\mathbf{k}}^{\dagger} & =u_{\mathbf{k}} \hat{b}_{-\mathbf{k}}^{\dagger}+v_{\mathbf{k}} \hat{b}_{\mathbf{k}}
\end{aligned}
$$

when $u_{\mathbf{k}}$ and $v_{\mathbf{k}}$ are chosen appropriately. The new operators $\hat{b}_{\mathbf{k}}$ and $\hat{b}_{\mathbf{k}}^{\dagger}$ satisfy the commutation relations for Bose field operators when

$$
u_{\mathbf{k}}^{2}-v_{\mathbf{k}}^{2}=1
$$


and with no loss of generality we can then write

$$
u_{\mathrm{k}}=\frac{1}{\sqrt{1-A_{\mathrm{k}}^{2}}}, \quad v_{\mathrm{k}}=\frac{A_{\mathrm{k}}}{\sqrt{1-A_{\mathrm{k}}^{2}}}
$$

It is straightforward to show that for (6.40) to be diagonalised $A_{\mathbf{k}}$ must satisfy the condition

$$
A_{\mathbf{k}}=\frac{1}{U_{0} n}\left[-\left(\epsilon_{\mathbf{k}}^{0}+U_{0} n\right)+\sqrt{\epsilon_{\mathbf{k}}^{0}\left(\epsilon_{\mathbf{k}}^{0}+2 U_{0} n\right)}\right]
$$

where $n=N_{0} / V$ is the density. The resulting Hamiltonian

$$
\hat{H}_{\text {Bog }}=E_{0}+\sum_{\mathbf{k} \neq 0} \epsilon_{\mathbf{k}} \hat{b}_{\mathbf{k}}^{\dagger} \hat{b}_{\mathbf{k}}
$$

is diagonal with a constant $E_{0}$ and where the quasiparticle excitations have the energy spectrum

$$
\epsilon_{\mathbf{k}}=\sqrt{\epsilon_{\mathbf{k}}^{0}\left(\epsilon_{\mathbf{k}}^{0}+2 U_{0} n\right)}
$$

in terms of the single particle energy

$$
\epsilon_{\mathbf{k}}^{0}=\frac{\hbar^{2} k^{2}}{2 m}
$$

In the Bogoliubov approximation, the Bose field operator can be expanded as

$$
\begin{aligned}
\hat{\Psi}(\mathbf{x}, t) & =e^{-i \mu t / \hbar}\left(\Psi_{0}(\mathbf{x})+\delta \hat{\varphi}(\mathbf{x}, t)\right) \\
& =e^{-i \mu t / \hbar}\left(\Psi_{0}(\mathbf{x})+\sum_{k} U_{k}(\mathbf{x}, t) \hat{b}_{\mathbf{k}}(0)+V_{k}^{*}(\mathbf{x}, t) \hat{b}_{\mathbf{k}}^{\dagger}(0)\right)
\end{aligned}
$$

Note that the time-dependence of each mode is fully contained in the mode functions $U_{k}(\mathbf{x}, t)$ and $V_{k}(\mathbf{x}, t)$. When the Hamiltonian is time-independent (eg. $U$ constant) the time-dependence is purely oscillatory and the number of quasiparticles in each mode $\left\langle\hat{b}_{\mathbf{k}}^{\dagger} \hat{b}_{\mathbf{k}}\right\rangle$ is a constant of the motion.

Alternatively when we linearise the density-phase representation of the field operator we find

$$
\delta \hat{\varphi}(\mathbf{x}, t) \approx \sqrt{n_{0}}\left(\frac{\hat{n}_{1}}{2 n_{0}}+i \hat{\theta}_{1}\right)
$$

If $\hat{n}_{1}$ and $\hat{\theta}_{1}$ are Hermitian we can write

$$
\hat{n}_{1}=\sqrt{n_{0}}\left(\delta \hat{\varphi}+\delta \hat{\varphi}^{\dagger}\right)
$$

and

$$
\hat{\theta}_{1}=\frac{1}{2 \sqrt{n_{0}} i}\left(\delta \hat{\varphi}-\delta \hat{\varphi}^{\dagger}\right)
$$


The commutation relations for the field operator (1.5) can be used to show that the density and phase fluctuations satisfy the commutation relation

$$
\left[\hat{n}_{1}(\mathbf{x}), \hat{\theta}_{1}\left(\mathbf{x}^{\prime}\right)\right]=i \delta\left(\mathbf{x}-\mathbf{x}^{\prime}\right)
$$

Using the mode expansion (6.11) we expand the field $\theta_{1}$ in Fourier (plane-wave) modes as

$$
\begin{aligned}
\hat{\theta}_{1} & =\sum_{\mathbf{k}}\left[\chi_{\mathbf{k}}(\mathbf{x}) \hat{b}_{\mathbf{k}}(t)+\chi_{\mathbf{k}}^{*}(\mathbf{x}) \hat{b}_{\mathbf{k}}^{\dagger}(t)\right] \\
& =\frac{1}{\sqrt{V}} \sum_{\mathbf{k}}\left[e^{i \mathbf{k} \cdot \mathbf{x}} \chi_{\mathbf{k}} \hat{b}_{\mathbf{k}}(t)+e^{-i \mathbf{k} \cdot \mathbf{x}} \chi_{\mathbf{k}}^{*} \hat{b}_{\mathbf{k}}^{\dagger}(t)\right]
\end{aligned}
$$

and similarly for $n_{1}$

$$
\begin{aligned}
\hat{n}_{1} & =\sum_{\mathbf{k}}\left[n_{\mathbf{k}}(\mathbf{x}) \hat{b}_{\mathbf{k}}(t)+n_{\mathbf{k}}^{*}(\mathbf{x}) \hat{b}_{\mathbf{k}}^{\dagger}(t)\right] \\
& =\frac{1}{\sqrt{V}} \sum_{\mathbf{k}}\left[e^{i \mathbf{k} \cdot \mathbf{x}} n_{\mathbf{k}} \hat{b}_{\mathbf{k}}(t)+e^{-i \mathbf{k} \cdot \mathbf{x}} n_{\mathbf{k}}^{*} \hat{b}_{\mathbf{k}}^{\dagger}(t)\right]
\end{aligned}
$$

The commutation relation (6.52) thus reduces to a Wronskian type condition for the normalisation of the modes:

$$
n_{k} \chi_{k}^{*}-n_{k}^{*} \chi_{k}=i
$$

We can expand $\delta \hat{\varphi}, \hat{n}_{1}$ and $\hat{\theta}_{1}$ in the same set of plane wave modes, using the mode expansions (6.53) and (6.54) and $U_{k}(\mathbf{x}, t)=u_{k}(t) e^{i \mathbf{k} \cdot \mathbf{x}} / \sqrt{V}$ and $V_{k}(\mathbf{x}, t)=v_{k}(t) e^{i \mathbf{k} \cdot \mathbf{x}} / \sqrt{V}$, so that the Fourier components are

$$
\begin{aligned}
& u_{k}(t)=\frac{1}{2 \sqrt{n_{0}}} n_{k}(t)+i \sqrt{n_{0}} \chi_{k}(t) \\
& v_{k}(t)=\frac{1}{2 \sqrt{n_{0}}} n_{k}(t)-i \sqrt{n_{0}} \chi_{k}(t)
\end{aligned}
$$

Clearly these mode functions are consistent with the requirement that the Bogoliubov modes are normalised by $\left|u_{k}\right|^{2}-\left|v_{k}\right|^{2}=1$. We note that these are general expressions, which are valid within the linearised theory of excitations no matter what form the mode functions take. That is, they are valid for arbitrary forms of the scaling function $b(t)$ so long as the mode functions $n_{k}(t)$ and $\chi_{k}(t)$ can be found. 


\subsubsection{Bogoliubov modes - Minkowski spacetime}

Using (6.30) with the mode expansions (6.53) and (6.54), the commutation relation (6.52) is satisfied when the mode functions take the form

$$
\begin{gathered}
\chi_{\mathbf{k}}=\frac{1}{2 \sqrt{n_{0}}} \sqrt{\frac{\epsilon_{k}}{\epsilon_{k}^{0}}} e^{-i \omega_{\mathbf{k}} t} \\
n_{\mathbf{k}}=i \sqrt{n_{0}} \sqrt{\frac{\epsilon_{k}^{0}}{\epsilon_{k}}} e^{-i \omega_{\mathbf{k}} t}
\end{gathered}
$$

These are the positive-frequency solutions for a time-independent Hamiltonian — that is, when when $U$ is constant - and correspond to an effective spacetime geometry that is Minkowski flat. The mode functions (6.56) and (6.57) can therefore be written

$$
\begin{array}{r}
\left|u_{k}\right|=\frac{1}{2}\left(\sqrt{\frac{\epsilon_{k}}{\epsilon_{k}^{0}}}+\sqrt{\frac{\epsilon_{k}^{0}}{\epsilon_{k}}}\right) \\
\left|v_{k}\right|=\frac{1}{2}\left(\sqrt{\frac{\epsilon_{k}}{\epsilon_{k}^{0}}}-\sqrt{\frac{\epsilon_{k}^{0}}{\epsilon_{k}}}\right)
\end{array}
$$

\subsubsection{Acoustic modes - Minkowski spacetime}

To facilitate the computation of particle production we again consider the acoustic approximation, which is valid for low momenta when $\hbar^{2} k^{2} / 2 m \ll U n_{0}$ so that the quantum pressure term can be neglected. In this case (6.30) reduces to

$$
n_{\mathbf{k}}=-\frac{\hbar}{U} \partial_{t} \chi_{\mathbf{k}}
$$

The mode functions are then given by

$$
\chi_{k}=\sqrt{\frac{U}{2 \hbar \omega_{k}}} e^{-i \omega_{k} t}
$$

and

$$
n_{k}=i \sqrt{\frac{\hbar \omega_{k}}{2 U}} e^{-i \omega_{k} t}
$$

Using the general expressions (6.56) and (6.57) the mode functions are given by

$$
\left|u_{k}\right|=\frac{1}{2}\left(\sqrt{\frac{\hbar \omega_{k}}{2 U n_{0}}}+\sqrt{\frac{2 U n_{0}}{\hbar \omega_{k}}}\right)
$$

and

$$
\left|v_{k}\right|=\frac{1}{2}\left(\sqrt{\frac{\hbar \omega_{k}}{2 U n_{0}}}-\sqrt{\frac{2 U n_{0}}{\hbar \omega_{k}}}\right)
$$




\subsubsection{Quasiparticle production}

The concept of particle production in our analogue model can be made explicit in terms of the Bogoliubov theory outlined above. To calculate the quasiparticle number in each mode after a finite expansion of interval $t$ it is necessary to project into the Bogoliubov basis that instantaneously diagonalises the many-body Bose Hamiltonian at $t$. From a practical viewpoint, this measure of particle production is correct if the scaling function $b\left(t^{\prime}\right)$ is constant for times $t^{\prime}>t$. In what follows we take the initial condition as the quasiparticle vacuum at $t=0$ so that $\hat{b}_{\mathbf{k}}(0)|0\rangle=0$ and therefore $N_{k}(0)=0$. Here the mode functions are determined by the solutions to the timeindependent case with $U(t)=U_{0}$, which yields the mode functions for a Minkowski spacetime. With this premise, we now demonstrate how to calculate the particle production for each mode for a given expansion. The Bogoliubov theory of a weakly interacting Bose gas predicts a nonzero depletion even at zero temperature; the real particle annihilation operator is given by the time-dependent canonical transformation

$$
\hat{a}_{\mathbf{k}}(t)=u_{k}^{\exp }(t) \hat{b}_{\mathbf{k}}(0)+v_{k}^{\exp *}(t) \hat{b}_{-\mathbf{k}}^{\dagger}(0)
$$

where $u_{k}^{\exp }$ and $v_{k}^{\exp }$ are solutions for the mode functions during the expansion - these must coincide with Minkowski mode functions at $t=0$ with $U(t)=U_{0}$. The projection into the Bogoliubov basis at $t$ is

$$
\hat{b}_{\mathbf{k}}(t)=u_{k}^{\text {out* } *}(t) \hat{a}_{\mathbf{k}}(t)-v_{k}^{\text {out* } *}(t) \hat{a}_{-\mathbf{k}}^{\dagger}(t)
$$

where $u_{k}^{\text {out }}$ and $u_{k}^{\text {out }}$ are given using (6.56) and (6.57) and the Minkowski mode solutions (6.62) and (6.63) with $U(t)=U_{0} / X$ in terms of of the expansion $X$. The particle production in each mode at time $t$ is then given by

$$
\begin{aligned}
N_{k}(t)= & \left\langle\hat{b}_{\mathbf{k}}^{\dagger}(t) \hat{b}_{\mathbf{k}}(t)\right\rangle \\
= & \left|u_{k}^{\text {out } *}(t) v_{k}^{\text {exp } *}(t)-v_{k}^{\text {out } *}(t) u_{k}^{\exp *}(t)\right|^{2} \\
= & \left(\left|u_{k}^{\text {out }}(t)\right|^{2}+\left|v_{k}^{\text {out }}(t)\right|^{2}\right)\left(\frac{1}{4 n_{0}}\left|n_{k}^{\exp }(t)\right|^{2}+n_{0}\left|\chi_{k}^{\exp }(t)\right|^{2}\right) \\
& -2\left|u_{k}^{\text {out }}(t)\right|\left|v_{k}^{\text {out }}(t)\right|\left(\frac{1}{4 n_{0}}\left|n_{k}^{\exp }(t)\right|^{2}-n_{0}\left|\chi_{k}^{\text {exp }}(t)\right|^{2}\right)-\frac{1}{2}
\end{aligned}
$$

where we have used (6.56) and (6.57). Evidently, the central task is to solve the field equation (5.17) for the mode functions $u_{k}^{\exp }$ and $v_{k}^{\exp }$ for a given expansion $b(t)$. This procedure will be applied in section 6.5.3 to calculate the quasiparticle production for the case of de Sitter expansion (in the acoustic approximation), and in section 6.6.1 for the case of sudden expansion (with highfrequency dispersion). 


\subsection{Quasiparticle production in acoustic approximation}

We presently provide analytic solutions for quasiparticle production in the acoustic approximation for three different expansion scenarios: (i) Sudden transition; (ii) tanh expansion; and (iii) de Sitter expansion. Scenarios (i) and (ii) both have asymptoticly flat in and out regions, so the calculation of particle production follows the standard procedure from section 6.3. Scenario (iii) however does not have asymptoticly flat in and out regions for a finite time expansion, and therefore we must resort to the Bogoliubov theory of the previous section to calculate the particle production.

\subsubsection{Sudden transition $(2+1$ dimensions $)$}

A simple example of particle production is given by the limiting case of a sudden expansion. Here, the interaction is instantaneously switched from $U$ to $U / X$ at some time $\tilde{t}_{0}$ which we take as $\tilde{t}_{0}=0$ for convenience. The scaling function is given by

$$
b(\tilde{t})=1-\left(1-\frac{1}{X}\right) H(\tilde{t})
$$

where $H$ is the Heaviside step function. The case of a particle production for a sudden transition has been previously explored by Jacobson for a parametric oscillator [166].

Using the normalisation condition (6.13) the positive frequency solutions to (6.27) are given by

$$
\chi_{k}^{\text {in } / \text { out }}=\frac{1}{\sqrt{2 \tilde{\omega}_{\mathbf{k}}^{\text {in } / \text { out }}}} e^{-i \tilde{\omega}_{\mathbf{k}}^{\text {in } / \text { out }} \tilde{t}}
$$

It is straightforward to calculate the Bogoliubov coefficients by applying the boundary conditions from (6.12) and its first derivative for the Minkowski in and out modes at $t_{0}=0$. We find

$$
\alpha_{\mathbf{k}}=\frac{1}{2}\left(\sqrt{\frac{\tilde{\omega}_{\mathbf{k}}^{\text {in }}}{\tilde{\omega}_{\mathbf{k}}^{\text {out }}}}+\sqrt{\frac{\tilde{\omega}_{\mathbf{k}}^{\text {out }}}{\tilde{\omega}_{\mathbf{k}}^{\text {in }}}}\right)
$$

and

$$
\beta_{\mathbf{k}}=\frac{1}{2}\left(\sqrt{\frac{\tilde{\omega}_{\mathbf{k}}^{\text {in }}}{\tilde{\omega}_{\mathbf{k}}^{\text {out }}}}-\sqrt{\frac{\tilde{\omega}_{\mathbf{k}}^{\text {out }}}{\tilde{\omega}_{\mathbf{k}}^{\text {in }}}}\right)
$$

so that

$$
N_{\mathbf{k}}^{\text {out }}=\left|\beta_{\mathbf{k}}\right|^{2}=\frac{1}{4}\left(\sqrt{\frac{\tilde{\omega}_{\mathbf{k}}^{\text {in }}}{\tilde{\omega}_{\mathbf{k}}^{\text {out }}}}-\sqrt{\frac{\tilde{\omega}_{\mathbf{k}}^{\text {out }}}{\tilde{\omega}_{\mathbf{k}}^{\text {in }}}}\right)^{2}
$$

Further noting for an expansion $X$ that $\tilde{\omega}_{\mathbf{k}}^{\text {out }} / \tilde{\omega}_{\mathbf{k}}^{\text {in }}=1 / \sqrt{X}$, the particle production is then

$$
N_{\mathbf{k}}^{\text {out }}=\frac{1}{4}\left(X^{1 / 4}-X^{-1 / 4}\right)^{2}
$$


which yields, for example: $N_{\mathrm{k}}^{\text {out }} \approx 0.4$ for $X=10 ; N_{\mathrm{k}}^{\text {out }} \approx 2$ for $X=100$; and $N_{\mathrm{k}}^{\text {out }} \approx 11$ for $X=2000$.

Equation 6.74 provides an upper limit for the particle production in each mode. This quantity does not depend on mode number - a feature that reflects the fact that all modes experience a sudden change in the effective spacetime geometry. We shall see in section 6.3.2 that including the quantum pressure term (ie. nonlinear dispersion) in our formulation leads to suppressed particle production for increasing $|\mathbf{k}|$.

It should be noted that the sudden transition corresponds to a delta function for the Hubble parameter $H$ at $\tilde{t}=0$; this is physically unfeasible as any change in the s-wave scattering length via a Feshbach resonance would require a finite time in practice [194]; moreover, a very rapid change in the scattering length is not possible since, in this case, the GPE would no longer be valid [109] (ie. the low momentum approximation for the T-matrix scattering potential is no longer valid). In spite of this, the sudden transition still provides a useful prediction for comparison with the results of the classical field simulations.

\subsection{2 tanh expansion $(2+1$ dimensions $)$}

One non-trivial form of the metric tensor for which the particle production can be calculated analytically is the case of a tanh function expansion, with asymptotically flat in and out regions. This was first considered by Bernard and Duncan [198] and Birrel and Davies [121] for a massive scalar field, and then by Barcelo et al. [109] for a massless scalar field. Similarly to [109] we consider the case of tanh expansion, but for $2+1$ dimensions.

In particular (6.27) can be solved exactly when the auxiliary factor $\Lambda$ has the time-dependence

$$
\Lambda^{3}(\tilde{t})=\frac{\Lambda_{i}^{3}+\Lambda_{f}^{3}}{2}+\frac{\Lambda_{f}^{3}-\Lambda_{i}^{3}}{2} \tanh \left(\frac{\tilde{t}}{\tilde{t}_{s}}\right)
$$

for some time constant $\tilde{t}_{s}$ that determines the rate of expansion. Noting that

$$
\Lambda^{3}=\Omega_{0}^{2} \frac{1}{b(\tilde{t})}
$$

the scaling function with respect to auxiliary time is

$$
\begin{aligned}
b(\tilde{t}) & =\Omega_{0}^{2}\left[\frac{\Lambda_{0}^{3}+\Lambda_{f}^{3}}{2}+\frac{\Lambda_{f}^{3}-\Lambda_{0}^{3}}{2} \tanh \left(\frac{\tilde{t}}{\tilde{t}_{s}}\right)\right]^{-1} \\
& =2\left[1+X+(X-1) \tanh \left(\frac{\tilde{t}}{\tilde{t}_{s}}\right)\right]^{-1}
\end{aligned}
$$

for an expansion $X$; we have implicitly assumed $b\left(\tilde{t}_{i}\right)=1$. We can also write

$$
\begin{aligned}
t & =\frac{\Omega_{0}}{c_{0}} \int \frac{1}{b(\tilde{t})} d \tilde{t} \\
& =\frac{\Omega_{0}}{2 c_{0}}\left[(1+X) \tilde{t}+(X-1) \tilde{t}_{s} \log \left(\cosh \left(\frac{\tilde{t}}{\tilde{t}_{s}}\right)\right)\right]
\end{aligned}
$$


This is not easy to invert but $b(\tilde{t})$ and $t(\tilde{t})$ define a parametric curve for $b(t)-$ this relation is required for implementing a tanh expansion in laboratory time simulations. With the conformal factor given by (6.75) it is possible to calculate the particle production in each mode exactly. Using the result in [109] we get

$$
N_{\mathbf{k}}^{\text {out }}=\frac{\sinh ^{2}\left[\pi k \tilde{t}_{s} \Omega_{0}(\sqrt{X}-1) / 2\right]}{\sinh \left[\pi k \tilde{t}_{s} \Omega_{0}\right] \sinh \left[\pi k \tilde{t}_{s} \Omega_{0} \sqrt{X}\right]}
$$

Note, in the limit $\tilde{t}_{s} \rightarrow 0$ this reduces to the sudden transition result (6.73) as expected.

\subsection{3 de Sitter universe $(2+1$ dimensions $)$}

We outline the particle production for the case of a de Sitter universe in the acoustic approximation.

The case of the de Sitter universe is particularly relevant in cosmology. The inflationary model of the early universe is thought to include a de Sitter phase of rapid expansion which accounts for the inhomogeneities observed in the present universe [199]. The de Sitter spacetime is a solution to the Einstein's field equations with a positive cosmological constant, and has a high degree of symmetry. It has been shown that an observer moving in a time-like geodesic will measure a thermal spectrum - this being a manifestation of the Hawking effect. This result was first derived by Gibbons and Hawking using the path integral formalism [200] and has been subsequently verified by applying the method of Bogoliubov mode mixing [201].

\section{Scaling function}

To map the FRW analogue model to a de Sitter spacetime, the scaling function for the scattering length in laboratory time (which is equivalent to proper time for two dimensions) is of the form

$$
b(t)=e^{-t / t_{s}}
$$

with the scaling unit $t_{s}$ that determines the rate of expansion. In this case the Hubble parameter (6.17) is given by $H=1 / 2 t_{s}$.

We further consider the transformation to so-called conformal time (denoted $\eta$ ) by $d \eta=$ $\sqrt{b(t)} d t$. In this case we get

$$
\eta=-2 t_{s} e^{-t / 2 t_{s}}, \quad t \geq 0
$$

The following limits are evident: (i) $\eta=-2 t_{s}$ for $t=0$; and (ii) $\eta \rightarrow 0$ as $t \rightarrow+\infty$. We also have:

$$
b(\eta)=\left(\frac{\eta}{2 t_{s}}\right)^{2}, \quad-2 t_{s} \leq \eta \leq 0
$$




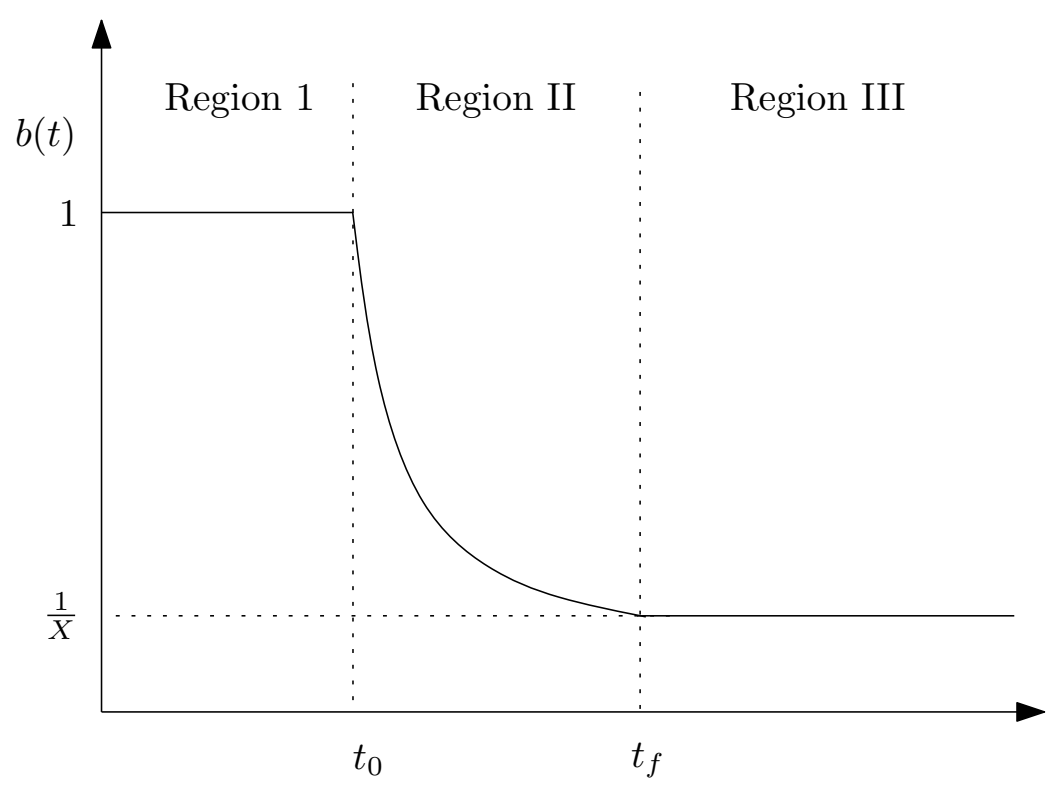

Figure 6.1: Schematic of de Sitter expansion for the FRW analogue model; for region I $\left(t<t_{0}\right)$, there is no expansion and the mode solutions are those of a Minkowski spacetime with $U=U_{0}$; for region II $\left(t_{0}<t<t_{f}\right)$, there is a de Sitter type expansion and the mode solutions are nontrivial; finally for region III $\left(t>t_{f}\right)$ the expansion is turned off and the mode solutions are those of a Minkowski spacetime with $U=U_{0} / X$.

\section{Mode solutions}

The field equation (6.22) then yields the second-order differential equation for the mode functions

$$
\frac{\partial^{2} \chi_{\mathbf{k}}}{\partial \eta^{2}}-\frac{1}{\eta} \frac{\partial \chi_{\mathbf{k}}}{\partial \eta}+c_{0}^{2} k^{2} \chi_{\mathbf{k}}=0
$$

This is a Bessel equation and the solution [202, equation 9.1.52] is given in terms of Bessel functions of the first and second kind ${ }^{4}$

$$
\chi_{\mathbf{k}}=A_{k} \eta J_{1}\left(-\omega_{k}(0) \eta\right)+B_{k} \eta Y_{1}\left(-\omega_{k}(0) \eta\right)
$$

for some undetermined constants $A_{k}$ and $B_{k}$; we have defined the frequency $\omega_{k}(0)=c_{0} k$ at $t=0$. Using (6.63) and [202, equation 9.1.27] it can be shown that the density fluctuation mode function is given by

$$
n_{k}=-2 t_{s} \frac{\hbar \omega_{k}(0)}{U_{0}}\left[A_{k} J_{0}\left(-\omega_{k} \eta\right)+B_{k} Y_{0}\left(-\omega_{k} \eta\right)\right]
$$

So that our analogue model corresponds to the classical field simulations we have run we require that $b(t)=1$ for $t \leq 0-$ ie. $\eta \leq-2 t_{s}$. Thus $t=0$ (or $\eta=-2 t_{s}$ ) corresponds to the in region with Minkowski mode functions. The limit $t \rightarrow \infty$ (or $\eta \rightarrow 0$ ) is the out region.

\footnotetext{
${ }^{4}$ With no loss of generality (as the Bessel equation is even in $\eta$ ), the arguments of $J_{1}$ and $Y_{1}$ are taken to be negative with respect to $\eta$ but positive overall in the region of interest - this facilitates computation as the Bessel functions are then real quantities.
} 


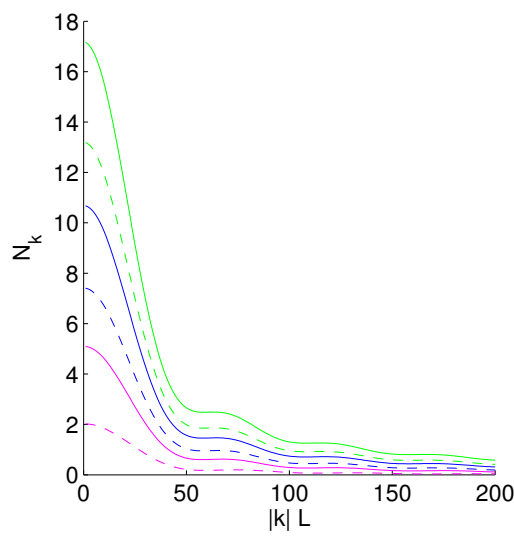

(a) $\bar{t}_{s}=1 \times 10^{-4}$
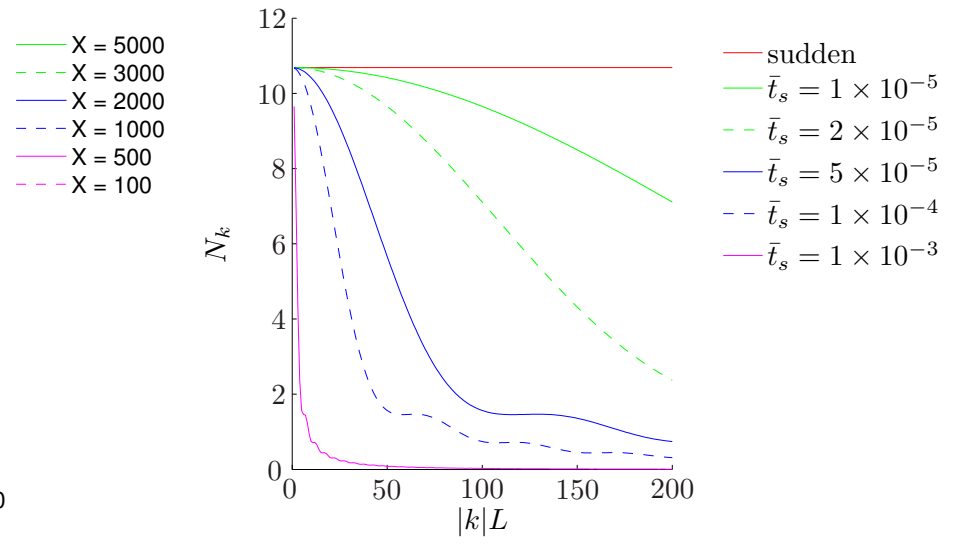

(b) $X=2000$

Figure 6.2: Particle production for a de Sitter spacetime in the FRW analogue model: (a) $\bar{t}_{s}=$ $1 \times 10^{-4}$ and a range of expansions $X$ as shown; (b) $X=2000$ and a range of expansion rates $\bar{t}_{s}$ as shown. The dimensionless scaling unit is $\bar{t}_{s}=t_{s} \hbar /\left(m L^{2}\right)$. In both cases the nonlinearity is $U_{0} N_{0}=10^{5} \hbar^{2} /\left(m L^{2}\right)$ (dimensionless units are introduced in chapter 7).

The goal is to calculate the Bogoliubov coefficients $\alpha_{k}$ and $\beta_{k}$ between these two regions and thus determine the particle production. Our expectation is a thermal spectrum in the limit of large $t_{s}$ which corresponds to the adiabatic regime.

Using [202, equation 9.1.16] it can be shown by matching the Minkowski and de Sitter modes at $t=0$ that

$$
\begin{gathered}
A_{k}=\frac{\pi}{2} \sqrt{\frac{U_{0} \omega_{k}(0)}{2 \hbar}}\left(i Y_{1}\left(2 t_{s} \omega_{k}(0)\right)-Y_{0}\left(2 t_{s} \omega_{k}(0)\right)\right) \\
B_{k}=-\frac{\pi}{2} \sqrt{\frac{U_{0} \omega_{k}(0)}{2 \hbar}}\left(i J_{1}\left(2 t_{s} \omega_{k}(0)\right)-J_{0}\left(2 t_{s} \omega_{k}(0)\right)\right)
\end{gathered}
$$

It can easily be verified using [202, equation 9.1.16] that these mode solutions satisfy the normalisation condition (6.55).

\section{Particle production}

The lack of a (globally) time-like Killing vector for the de Sitter universe means it is not possible to unambiguously define a Fock vacuum at any time, and that therefore, the particle number of each mode after some expansion is observer dependent. However, for our analogue model, we circumvent this complication by associating an instantaneous Minkowski vacuum at each point in time - that is, we project into the quasiparticle basis that diagonalises the many body Hamiltonian to second order. This prescription has previously been outlined in section 6.4.4. Figure 6.1 shows the scaling factor for the relevant temporal regions.

To proceed, for brevity, we define $z \equiv-\omega_{k}(0) \eta=2 t_{s} \omega_{k}(0) e^{-t / 2 t s}$ and set $z_{0}=z(t=0)=$ $2 t_{s} \omega_{k}(0)$. Additionally we introduce $\lambda_{0}=\sqrt{2 U(0) n_{0} / \hbar \omega_{k}(0)}$. Using the mode solutions (6.84) 


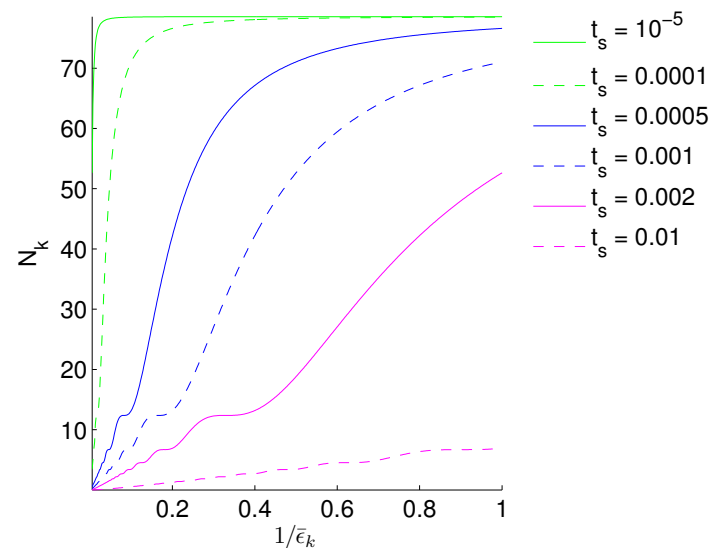

(a)

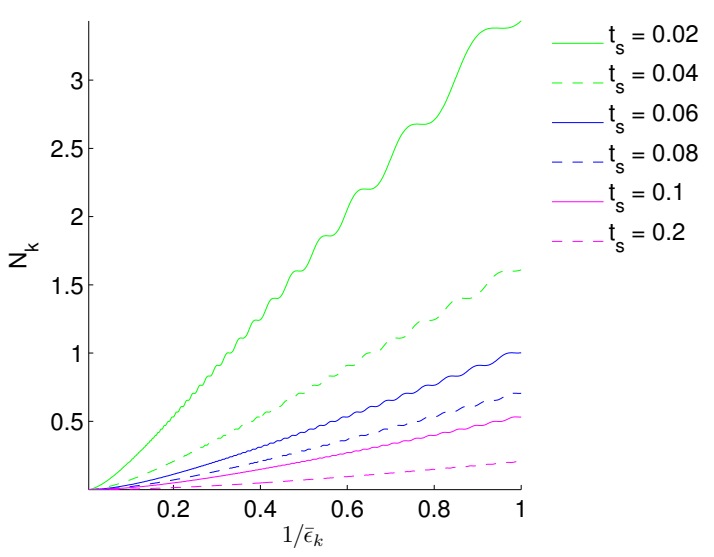

(b)

Figure 6.3: Equipartition of energy for a de Sitter spacetime in the FRW analogue model. In both cases the expansion is $X=1 \times 10^{5}$ and the nonlinearity is $U_{0} N_{0}=10^{5} \hbar^{2} /\left(m L^{2}\right)$. The dimensionless energy is $\bar{\epsilon}_{k}=\epsilon_{k} m L^{2} / \hbar^{2}$ (dimensionless units are introduced in chapter 7).

and (6.85) we then have

$$
\begin{aligned}
\frac{1}{4 n_{0}}\left|n_{k}^{d S}(t)\right|^{2}=\frac{1}{\lambda_{0}^{2}} \frac{\left(\pi t_{s} \omega_{k}(0)\right)^{2}}{4}[ & \left(Y_{1}^{2}\left(z_{0}\right)+Y_{0}^{2}\left(z_{0}\right)\right) J_{0}^{2}(z(t))+\left(J_{1}^{2}\left(z_{0}\right)+J_{0}^{2}\left(z_{0}\right)\right) Y_{0}^{2}(z(t)) \\
& \left.-2\left(J_{1}\left(z_{0}\right) Y_{1}\left(z_{0}\right)+J_{0}\left(z_{0}\right) Y_{0}\left(z_{0}\right)\right) J_{0}(z(t)) Y_{0}(z(t))\right]
\end{aligned}
$$

and

$$
\begin{aligned}
n_{0}\left|\chi_{k}^{d S}(t)\right|^{2}=\lambda_{0}^{2} \frac{\left(\pi t_{s} \omega_{k}(0)\right)^{2}}{4 X}[ & \left(Y_{1}^{2}\left(z_{0}\right)+Y_{0}^{2}\left(z_{0}\right)\right) J_{1}^{2}(z(t))+\left(J_{1}^{2}\left(z_{0}\right)+J_{0}^{2}\left(z_{0}\right)\right) Y_{1}^{2}(z(t)) \\
& \left.-2\left(J_{1}\left(z_{0}\right) Y_{1}\left(z_{0}\right)+J_{0}\left(z_{0}\right) Y_{0}\left(z_{0}\right)\right) J_{1}(z(t)) Y_{1}(z(t))\right]
\end{aligned}
$$

We can therefore use (6.68) to calculate the particle production explicitly - these results are shown in Figures 6.2 (a) and (b). In particular, Figure 6.2 (a) shows the particle production for a fixed rate of expansion $t_{s}=1 \times 10^{-4}$ and several different expansions $X$, whereas Figure 6.2 (b) shows the particle production for a fixed expansion $X=2000$ with several different rates of expansion $t_{s}$.

\section{Limits}

The particle production from the de Sitter expansion interpolates between two opposite limits: a sudden expansion for $t_{s} \rightarrow 0$, and an analytically tractable asymptotic limit for $1 \ll 2 t_{s} \omega_{k}(0) \ll$ $X^{1 / 2}$. 


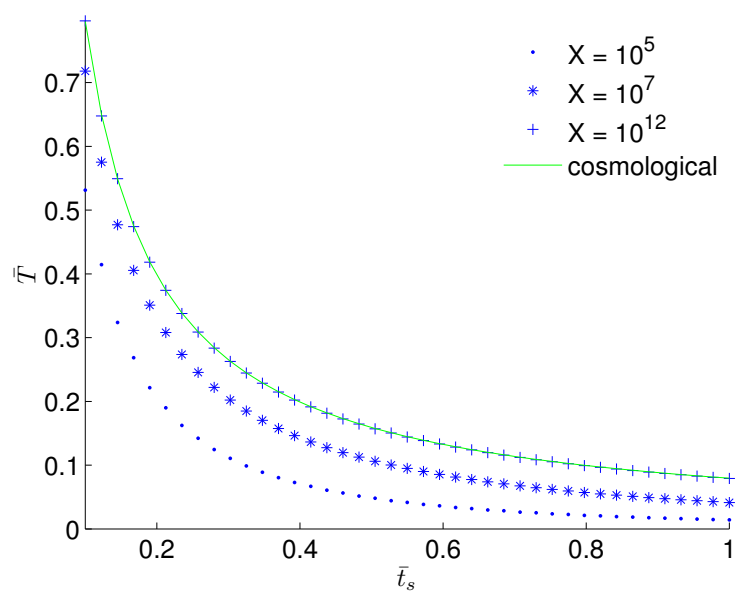

Figure 6.4: Temperature from linear fit to equipartition for three different expansions $X$ for the FRW analogue model. The cosmological result from (6.93) is shown by the green solid curve. The dimensionless temperature is $\bar{T}=k_{B} T \hbar^{2} /\left(m L^{2}\right)$. The dimensionless scaling unit is $\bar{t}_{s}=$ $t_{s} \hbar /\left(m L^{2}\right)$. The nonlinearity is $U_{0} N_{0}=10^{5} \hbar^{2} /\left(m L^{2}\right)$ (dimensionless units are introduced in chapter 7).

Sudden expansion: In this case we have the Minkowski mode functions:

$$
\begin{aligned}
\frac{1}{4 n_{0}}\left|n_{k}^{d s}(t)\right|^{2} & \rightarrow \frac{1}{4 \lambda_{0}^{2}} \\
n_{0}\left|\chi_{k}^{d S}(t)\right|^{2} & \rightarrow \frac{1}{4} \lambda_{0}^{2}
\end{aligned}
$$

and the particle production from (6.68) reduces to the expression (6.74) we found previously for a sudden expansion

$$
N_{k} \rightarrow \frac{1}{4}\left(X^{1 / 4}-X^{-1 / 4}\right)^{2}
$$

This behaviour can be clearly seen in Figure 6.2 (b) where the particle number approaches the sudden result for faster expansion rates (ie. smaller values of $t_{s}$ ).

\section{Asymptotic expansion:}

When the rate of expansion is sufficiently slow and in the limit of a very large expansion with $1 \ll 2 t_{s} \omega_{k}(0) \ll X^{1 / 2}$, the particle production approaches the usual cosmological result of a thermal spectrum [121], which in terms of $t_{s}$ is given by a temperature

$$
k_{B} T=\frac{1}{4 \pi t_{s}}
$$

This can be seen in Figures 6.3 (a) and (b) which shows a plot of $N_{k}$ vs $1 / \epsilon_{k}$ as calculated from (6.68) and (6.46) respectively. If the system is in thermal equlibrium, then such a plot should be linear according to equipartition of energy $N_{k} \epsilon_{k}=k_{B} T$. This is approximately valid when $1 \ll 2 t_{s} \omega_{k}(0) \ll X^{1 / 2}$, which is consistent with the condition for adiabatic 
expansion, where the system remains close to equilibrium throughout the expansion. Furthermore, Figure 6.4 shows the fitted temperature from (6.68) as a function of expansion rate $t_{s}$ for three different expansions $X$. For a very large expansion $X=1 \times 10^{12}$ the result closely agrees with the result from $(6.93)^{5}$.

\subsection{Quasiparticle production beyond the acoustic approximation}

In this section we consider quasiparticle production where the acoustic approximation is not enforced - we introduced this more general case in section 6.3.2. In general the usual calculations become unmanageable in this regime, and so, we present only two expansion scenarios here: We first calculate quasiparticle production for a sudden transition in section 6.6.1, for which an exact solution can be easily found; In section 6.6.2 we additionally consider the cyclic universe model, for which we are able to make some general statements with regards to quasiparticle production.

\subsubsection{Sudden Expansion}

The particle production for a sudden expansion can be calculated using the Bogoliubov theory from section 6.4. The scaling function is given by (6.69) with the substitution for laboratory time $\tilde{t} \rightarrow t$. The excitation of each mode is attributed to the quantum depletion corresponding to the initial vacuum state with nonlinearity $U_{0}$, projected into the Bogoliubov basis corresponding to the final nonlinearity $U_{0} / X$. We can thus calculate the number of Bogoliubov quasiparticles directly using (6.41) assuming the initial state is the Bogoliubov vacuum defined by $\hat{b}_{\mathbf{k}}^{\mathrm{in}}|0\rangle=0$. Using (6.68) the result is

$$
\begin{aligned}
N_{\mathbf{k}}^{\text {out }} & =\left(u_{\mathbf{k}}^{\text {out }} v_{\mathbf{k}}^{\text {in }}-v_{\mathbf{k}}^{\text {out }} u_{\mathbf{k}}^{\text {in }}\right)^{2} \\
& =\frac{\left(A_{\mathbf{k}}^{\text {in }}-A_{\mathbf{k}}^{\text {out }}\right)^{2}}{\left(1-A_{\mathbf{k}}^{\text {in } 2}\right)\left(1-A_{\mathbf{k}}^{\text {out } 2}\right)}
\end{aligned}
$$

where $A_{\mathbf{k}}^{\text {in }}$ and $A_{\mathbf{k}}^{\text {out }}$ is given by (6.44) with $U=U_{0}$ and $U=U_{0} / X$ respectively. The particle production from (6.95) is suppressed for modes of large momenta, as can be seen from (6.44) by the observation that $A_{\mathrm{k}}^{\text {in/out }} \rightarrow 0$ as $|\mathbf{k}| \rightarrow \infty$ - this is consistent with the preceding discussion of section 6.3.2.

This calculation can also be repeated using the usual methods of QFTCS as outlined in section 6.3 - similarly to the previous calculation for the sudden transition in the acoustic approximation outlined in section 6.5.1 — but instead by using the field equation (6.31), which includes nonlinear dispersion. The result is [204]

$$
N_{\mathbf{k}}^{\text {out }}=\left|\beta_{\mathbf{k}}\right|^{2}=\frac{1}{4}\left(\sqrt{\frac{\omega_{\mathbf{k}}^{\text {in }}}{\omega_{\mathbf{k}}^{\text {out }}}}-\sqrt{\frac{\omega_{\mathbf{k}}^{\text {out }}}{\omega_{\mathbf{k}}^{\text {in }}}}\right)^{2}
$$

${ }^{5} \mathrm{We}$ can relate this to the number of $e$-foldings, which is given by [203]

$$
N_{e}(t) \equiv \ln \left(\frac{a_{\mathrm{FRW}}(t)}{a_{\mathrm{FRW}}(0)}\right)=\ln \sqrt{X}
$$

to give $N_{e}\left(t_{f}\right) \approx 14$ for $X=1 \times 10^{12}$. 
This takes the same form as the result from the acoustic approximation given by (6.73). However, here $\omega_{\mathbf{k}}^{\text {in }}$ and $\omega_{\mathbf{k}}^{\text {out }}$ are the Bogoliubov mode frequencies given by (6.46), which includes nonlinear dispersion, for the in and out regions respectively.

It is straightforward to show that (6.95) and (6.96) agree exactly for all $|\mathbf{k}|$. We further note that for small $|\mathbf{k}|$, the Bogoliubov coefficients are $u_{k} \approx v_{k}$ and the Bogoliubov mode expansion coincides with the mode expansion for the quantised phase fluctuations (6.11). Therefore in this limit the acoustic result (6.74) agrees with (6.95).

\subsubsection{Cyclic universe $(2+1$ dimensions $)$}

The final scenario we consider is that of a cyclic (or oscillatory) universe. While models of a cyclic universe certainly exist in the literature [205], they are not as firmly established as inflationary models (such as the de Sitter universe). From a condensed matter point of view, an analogue model of a cyclic universe is interesting because it leads to parametric excitation of the quasiparticle modes, and in particular to parametric resonance [206]. Moreover, by taking $b\left(t_{f}\right)=b(t=0)$, a cyclic universe is an interesting counter-point to the case of a sudden transition: in a sudden transition the field does not evolve and any particle production is entirely attributed to a sudden change in the effective spacetime (ie. we project into a new quasiparticle basis that depends on the final nonlinearity $\left.U=U_{0} / X\right)$; however, in a cyclic universe model, the initial and final effective spacetimes are the same (so long as condensate does not evolve too far from the initial ground state) and particle production occurs due to parametric excitation only.

The scaling function can be expressed as

$$
b(t)=\frac{1}{X}+\frac{1}{2}\left(1-\frac{1}{X}\right)\left[\cos \left(\frac{2 \pi m t}{t_{f}}\right)+1\right]
$$

with $b(0)=1$ and where we have defined $m$ as the number of cycles of the oscillation (we note that $t_{s}=t_{f} / m$ is the period of each oscillation), and $X$ is now defined as the amplitude of the oscillation (rather than the final expansion).

\section{Parametric resonance}

The phenomena of parametric resonance has been previously investigated in Bose condensed systems [207,208], and in the context of an expanding universe model [209]. The condition for parametric resonance is close to $\omega(k)=\Omega / 2$ where $\Omega=2 \pi m / t_{f}$ is the driving frequency of some external parameter [206], in this case the nonlinearity. Using the Bogoliubov excitation spectrum $\epsilon(k)=\hbar \omega(k)$ from (6.46) then gives a simple estimate of the peak wave-vector for resonance:

$$
k_{\text {resonance }}=\left(\frac{2 m}{\hbar^{2}}\right)^{1 / 2}\left[\left(\left(U_{\text {ave }} N_{0}\right)^{2}+\left(\hbar \pi / t_{s}\right)^{2}\right)^{1 / 2}-U_{\text {ave }} N_{0}\right]^{1 / 2}
$$

where $U_{\text {ave }}=\frac{1}{2}(1+1 / X) U_{0}$ is the average nonlinearity during the evolution. However, it is worth noting that this result requires that $\Delta=1-1 / X$ from (6.97) is a perturbative parameter with $\Delta \ll 1$; we therefore henceforth refer to this as the perturbative resonance condition. 
The extension of the analysis to a strongly driven system $(\Delta \lesssim 1)$ has been considered in [207]. The general result is that the mode frequency region where parametric resonance occurs broadens as $\Delta$ increases; that is, for the parametric resonance peak broadens in momentum space with larger expansion $X$. 


\section{Chapter 7}

\section{The classical field method}

\subsection{Introduction}

Classical field methods, introduced in chapter 1, are a powerful tool for approximating the dynamics of quantum systems. Their application to Bose-Einstein condensates include the closely related methodologies of the finite temperature Gross-Pitaevskii equation [53, 54], the truncated Wigner approximation [34-37, 41, 42] and the positive-P method [34, 43].

Neglecting quantum and thermal fluctuations, the condensate dynamics are determined by the Gross-Pitaevskii equation (GPE) given by (1.8). This is derived from the Heisenberg equation of motion (1.6) for the field operator $\hat{\psi}(\mathbf{x})$, by replacing $\hat{\psi}(\mathbf{x})$ with the mean-field order parameter $\psi(\mathbf{x})=\langle\hat{\psi}(\mathbf{x})\rangle$, which is interpreted as the wavefunction of the condensate. This approximation arises from the assumption that the condensate is highly occupied so that $N_{0}=\int d \mathbf{x}\left\langle\hat{\psi}^{\dagger}(\mathbf{x}) \hat{\psi}(\mathbf{x})\right\rangle \gg 1$. In the classical field approximation this description is extended by also including the non-condensate modes from a low energy subspace of the system; these modes are to be considered classical in that they are highly populated - this is akin to the Bogoliubov approximation where the commutators can be neglected. We thus proceed by expanding the field operator in some basis

$$
\hat{\psi}(\mathbf{x}, t)=\sum_{\mathbf{k}} \phi_{\mathbf{k}}(\mathbf{x}) \hat{a}_{\mathbf{k}}(t)
$$

and replacing this in (1.6) by the classical field

$$
\psi(\mathbf{x}, t)=\sum_{\mathbf{k} \in C} \phi_{\mathbf{k}}(\mathbf{x}) \alpha_{\mathbf{k}}(t)
$$

where $\hat{a}_{\mathbf{k}} \rightarrow \alpha_{\mathbf{k}}$ for those modes where $N_{\mathbf{k}}=\left\langle\hat{a}_{\mathbf{k}}^{\dagger} \hat{a}_{\mathbf{k}}\right\rangle \gg 1$ is satisfied; we denote these modes in the low-energy subspace by $k \in C$. For a homogeneous Bose gas in a box with periodic boundary conditions the modes of the system (the eigenstates that diagonalise the single-particle Hamiltonian) are plane wave states

$$
\phi_{\mathbf{k}}(\mathbf{x})=\frac{1}{\sqrt{V}} e^{i \mathbf{k} \cdot \mathbf{x}}
$$


The low-energy subspace $C$ is then determined by a momentum cut-off, below which all modes are retained in the classical field. This is formalised by the use of a projector which is defined by its action on some function $f(\mathbf{x})$ as

$$
\hat{P}\{f(\mathbf{x})\}=\sum_{\mathbf{k} \in C} \phi_{\mathbf{k}}(\mathbf{x}) \int d \mathbf{x}^{\prime} \phi_{\mathbf{k}}^{*}\left(\mathbf{x}^{\prime}\right) f\left(\mathbf{x}^{\prime}\right)
$$

\section{Projected Gross-Pitaevskii equation}

Neglecting all modes orthogonal to $C$ the projected Gross-Pitaevskii equation (PGPE) is given by

$$
i \hbar \frac{\partial \psi(\mathbf{x})}{\partial t}=\hat{H}_{0} \psi(\mathbf{x})+U_{0} \hat{P}\left\{|\psi(\mathbf{x})|^{2} \psi(\mathbf{x})\right\}
$$

A projector is required for the following reasons:

1. The classical field approximation naturally divides the system into a coherent region, which is described by the propagation of a classical field, and an incoherent region which is neglected in the present formalism. In equilibrium, the system is then described by a microcanonical ensemble since particle numbers are conserved by the Hamiltonian (1.1).

2. While the finite size of the spatial grid inherently defines a momentum cut-off, the split operator Fourier methods used to propagate the classical field can introduce aliasing if a projector is not applied explicitly.

3. Because the PGPE uses a contact potential to describe two-body interactions, and such a description leads to ultraviolet divergences at large momenta, a cut-off is required.

\subsection{The truncated Wigner approximation}

A formal framework for the ideas outlined above is provided by the truncated Wigner approximation (TWA). We briefly outline the method but the reader is referred to [34-37,41,42] for further details.

The TWA is a phase space method originating from the representation of the density operator in terms of the Wigner function, which is familiar from quantum optics [30]. The master equation for the multimode density operator can be formally mapped to a third order differential equation for the Wigner function by the application of operator correspondences. The approximation involved in the TWA is to neglect the third order derivative terms, which become small for highly occupied modes. The resulting Fokker-Planck type equation has no diffusion term and is equivalent to evolving a classical field of the form (7.2) with the GPE with two crucial modifications:

1. Quantum vacuum fluctuations are included in the initial state by adding classical noise sampled from the Wigner distribution; the form of this noise depends on the Wigner function for the ground state of the system. For a Bose-Einstein condensate at $T=0$ the initial amplitude of each mode is a random Gaussian variable that is distributed according to the Wigner function for a coherent state. 
2. The moments of the the Wigner function give the expectation values for symmetrically ordered operators. In practice calculating the expectation value of an observable $O$ requires an ensemble average over many trajectories in phase space. We denote such an expectation value by $\langle O\rangle_{W}$.

\subsubsection{Initial states: vacuum noise}

The initial condition is given by superposition of the ground state and noise sampled from the Wigner distribution.

$$
\psi(\mathbf{x}, t=0)=\psi_{0}(\mathbf{x})+\delta \psi(\mathbf{x})
$$

We can expand the noise term via a Fourier transform as

$$
\eta(\mathbf{r})=\frac{1}{\sqrt{V}} \sum_{\mathbf{k} \neq 0} e^{i \mathbf{k} \cdot \mathbf{r}} \eta_{\mathbf{k}}
$$

\section{Bogoliubov vacuum}

Within the truncated Wigner approximation the initial vacuum state ${ }^{1}$ is prepared by specifying noise on each of the Bogoliubov modes:

$$
\delta \psi(\mathbf{x})=\sum_{k \neq 0}\left(U_{\mathbf{k}}(\mathbf{x}) \beta_{\mathbf{k}}+V_{\mathbf{k}}^{*}(\mathbf{x}) \beta_{\mathbf{k}}^{*}\right)
$$

where $U_{\mathbf{k}}(\mathbf{x})$ and $V_{\mathbf{k}}(\mathbf{x})$ are the plane wave modes with amplitudes $u_{\mathbf{k}}$ and $v_{\mathbf{k}}$ respectively (as defined in Sect. 6.4.1). $\beta_{\mathbf{k}}$ are complex random variables that obey the Gaussian statistics [34,55]:

$$
\begin{aligned}
\left\langle\beta_{\mathbf{p}} \beta_{\mathbf{q}}\right\rangle & =\left\langle\beta_{\mathbf{p}}^{*} \beta_{\mathbf{q}}^{*}\right\rangle=0 \\
\left\langle\beta_{\mathbf{p}}^{*} \beta_{\mathbf{q}}\right\rangle & =\frac{1}{2} \delta_{p, q}
\end{aligned}
$$

The initial state is thus constructed by populating the Bogoliubov modes with half a particle per mode according to the TWA prescription, for the initial nonlinearity $U_{0}$; for our cosmological model this corresponds to the instantaneous vacuum state (Minkowski vacuum) in laboratory time

\footnotetext{
${ }^{1}$ White noise vacuum:

Another choice for the initial vacuum is to add noise to the real particle modes of the system using

$$
\eta_{\mathbf{k}}=\frac{1}{\sqrt{V}} \int d \mathbf{r} e^{-i \mathbf{k} \cdot \mathbf{r}} \eta(\mathbf{r})
$$

where $\eta_{\mathbf{k}}$ are complex random variables that satisfy the following Gaussian statistics

$$
\begin{aligned}
\left\langle\eta_{\mathbf{p}} \eta_{\mathbf{p}}\right\rangle & =\left\langle\eta_{\mathbf{p}}^{*} \eta_{\mathbf{p}}^{*}\right\rangle=0 \\
\left\langle\eta_{\mathbf{p}}^{*} \eta_{\mathbf{q}}\right\rangle & =\frac{1}{2} \delta_{p, q}
\end{aligned}
$$

This choice for the initial state is a poor description of the many body ground state as the quantum depletion is not properly accounted for; in dynamics with the TWA this can therefore lead to heating as is evident by an transient thermalisation of the system $[34,35]$. In the limit of no interactions $(U=0)$ the Bogoliubov and white noise vacua coincide.
} 
at $t=0$.

The quantum and Wigner expectation values for the population of the $\mathrm{k}$ mode in the Bogoliubov quasiparticle basis are related by

$$
\left\langle\beta_{\mathbf{k}}^{*} \beta_{\mathbf{k}}\right\rangle_{W}=\left\langle\left\{\hat{b}_{\mathbf{k}}^{\dagger} \hat{b}_{\mathbf{k}}\right\}\right\rangle=\left\langle\hat{N}_{\mathbf{k}}\right\rangle+\frac{1}{2}
$$

where the brackets require that the symmetrised operator should be taken. The vacuum state therefore corresponds to half a particle per mode in the classical field.

\subsubsection{Validity of the TWA}

In the present application of the TWA, only the $\mathrm{k}=0$ condensate mode is macroscopically occupied, the other modes being initially unpopulated (for the quantum expectation value). The requirement that $N_{\mathrm{k}} \gg 1$ for each mode in the classical field is then violated. However a more detailed treatment of the validity of the TWA leads to the criterion that $N \gg M$ for a system of $N$ particles and $M$ modes [37]. This criterion has been made explicit by Norrie et al. as the requirement that the particle density exceeds the commutator for the restricted field operator [41]. It has been shown that for a homogeneous system these two criteria coincide [210]. Therefore the TWA can still be applied when most of the modes are unoccupied as long as the average particle density is sufficiently large.

Moreover the above choice for the initial state can lead to heating as is evident by an transient thermalisation of the system $[34,35]$. In this case the classical field dynamics deviate from the Bogoliubov theory valid for a weakly interacting gas; the system evolves to thermal equilibrium via the nonlinear interactions between Bogoliubov modes. This effect can be suppressed by evolving the classical field only for short times and by choosing a regime where system is weakly interacting (ie. $U$ small). This is an important consideration for our simulations where any thermalisation could obscure the effect of particle production.

\subsubsection{Quasiparticle number}

Following the discussion on Bogoliubov theory in section 6.4 the classical field for the homogeneous system can be expressed as

$$
\Psi(\mathbf{x}, t)=e^{-i \mu t / \hbar}\left(\Psi_{0}(\mathbf{x})+\frac{e^{i \mathbf{k} \cdot \mathbf{x}}}{\sqrt{V}} \sum_{k} u_{k} \beta_{-\mathbf{k}}(t)+v_{k} \beta_{\mathbf{k}}^{*}(t)\right)
$$


where the time-dependent amplitudes are given from (6.41) by ${ }^{2}$

$$
\beta_{\mathbf{k}}(t)=u_{k} \alpha_{\mathbf{k}}(t)-v_{k} \alpha_{-\mathbf{k}}^{*}(t)
$$

Referring to (7.14), the quasiparticle number in the TWA prescription is given by (where the expectation value is implicitly assumed)

$$
N_{\mathbf{k}}(t)=\left\langle\beta_{\mathbf{k}}^{*}(t) \beta_{\mathbf{k}}(t)\right\rangle_{W}-\frac{1}{2}
$$

We use this quantity to calculate the quasiparticle mode populations in our simulations; at each time the mode functions $u_{k}$ and $v_{k}$ are determined from (6.43) using nonlinearity $U(t)$. Hence this result is consistent with (6.68), as it requires projection into the quasiparticle basis that instantaneously diagonalises (to second order in quasiparticle operators) the many body Hamiltonian $(1.1)$.

\subsection{Numerical details}

\subsubsection{Dimensionless GPE}

Our "universe" is specified by a box with dimensions $L_{x}=\gamma_{x} L, L_{y}=\gamma_{y} L$ and $L_{z}=\gamma_{z} L$. In what follows we assume $\gamma_{x}=\gamma_{y}=1$ and with $\gamma_{z}$ strictly less than one as required by the quasi-two dimensional model. To facilitate numerical computation we introduce the dimensionless parameters

$$
\overline{\mathbf{x}}=\frac{\mathbf{x}}{L}, \quad \bar{\psi}=\psi \frac{L^{d / 2}}{\sqrt{N_{0}}}, \quad \bar{t}=t \frac{\hbar}{m L^{2}}
$$

With a time-dependent nonlinear interaction, the two-dimensional GPE (6.10) then takes the dimensionless form

$$
i \frac{\partial \bar{\psi}}{\partial \bar{t}}=\left[-\frac{1}{2} \bar{\nabla}^{2}+\bar{V}_{\mathrm{ext}}+C_{N L}(\bar{t})|\bar{\psi}|^{2}\right] \bar{\psi}
$$

The dimensionless potential is

$$
\bar{V}_{\mathrm{ext}}=\frac{V_{\mathrm{ext}} m L^{2}}{\hbar^{2}}
$$

\footnotetext{
${ }^{2}$ Time-dependent Bogoliubov transformation:
}

The equation of motion (ie. the GPE in momentum space) for the operators (6.41) also includes a phase factor from the evolution of the condensate mode. Therefore in practice, to project into the Bogoliubov basis requires the timedependent transformation

$$
\beta_{\mathbf{k}}(t)=u_{\mathbf{k}} \alpha_{\mathbf{k}}(t) \frac{\alpha_{0}^{*}}{\left|\alpha_{0}\right|}-v_{\mathbf{k}} \alpha^{*}{ }_{\mathbf{k}}(t) \frac{\alpha_{0}}{\left|\alpha_{0}\right|}
$$

where the condensate phase factor is given by $\alpha_{0} /\left|\alpha_{0}\right|=e^{-i \mu t / \hbar}$. 
whereas the effective nonlinearity is (integrating over the $z$ direction for the quasi-two dimensional geometry)

$$
C_{N L}(\bar{t})=\frac{U_{2 \mathrm{D}} b(\bar{t}) N_{0} m}{\hbar^{2}}=\frac{4 \pi a(\bar{t}) N_{0}}{L_{z}}
$$

Note that the wave function is normalised to unity here. The corresponding dimensionless speed of sound is

$$
\bar{c}=\frac{m L}{\hbar} c=\sqrt{C_{N L}}
$$

For completeness, we note the Bogoliubov excitation spectrum (6.46) in dimensionless units is given by

$$
\epsilon_{\bar{k}}=\sqrt{\frac{\bar{k}^{2}}{2}\left(\frac{\bar{k}^{2}}{2}+2 C_{N L}\right)}
$$

We take $\bar{V}_{\text {ext }}=0$ for the homogeneous system. In dimensionless units the spatial coordinates span the region $-\frac{1}{2} \leq \bar{x} \leq \frac{1}{2}$. For convenience we henceforth drop the bar notation (unless otherwise specified).

\subsubsection{The projected RK4IP algorithm}

Equation (7.20) is propagated using the 4th order Runge Kutta algorithm in the interaction picture [211]. For the results presented here the time step was chosen so that the total normalisation change during each trajectory was $\Delta$ norm $\leq 10^{-9}$ (for our choice of total particle number, this corresponds to a total loss or gain of much less than one particle for the entire field). We take the condensate volume in dimensionless units as the region $-\frac{1}{2} \leq \mathbf{x} \leq \frac{1}{2}$. The field is discretised on a grid of $128 \times 128$ points. The projector retains all modes with $|k| \leq 32 \times 2 \pi$. The classical field then contains $M=3209$ modes.

In practice, the mode populations $N_{\mathbf{k}}\left(k_{x}, k_{y}\right)$ were resampled on polar coordinates $|k|$ and $\phi$ and then averaged over angle ${ }^{3}$.

Further details of the numerical algorithm are provided in Appendix B.

\subsection{Suitable parameter regime}

It is appropriate, at this point, to determine a viable set of parameters for the classical field simulations. The choice of simulation parameters is constrained by three main factors:

- The criterion for the validity of the classical field method (ie. the truncated Wigner approximation).

\footnotetext{
${ }^{3}$ Technical note: when averaging over angle in momentum space we have sampled $|k|$ at 32 points; the first and last points are excluded from the resulting distribution since the linear interpolating function used for resampling is inaccurate (1) when $|k| \approx 0$ and the mode population is large; and (2) at the edge of the projected region where the mode populations are explicitly set to zero.
} 
- The requirement that all modes of the system are in the phononic regime at the start of the simulation. In this regime the particle production is significant.

- A set of parameters that are experimentally relevant.

The criteria for the validity of the TWA, has been discussed in section 7.2.2. In particular, for our simulations, we are required to choose a condensate population with $N_{0} \gg M=3209$. Noting that in our simulations the classical field is normalised to 1 , the requirement that the system is weakly interacting is satisfied when the nonlinearity $C_{N L}$ is small compared with the condensate population $N_{0}$.

Furthermore we wish to investigate a regime where a significant fraction of the modes are phononic (as determined by $k<1 / \xi$ ) so that we can compare our results with the analytic calculations in the acoustic approximation. In computational units the phonon to free-particle cross-over is determined by $\bar{k}_{c}^{2} / 2=C_{N L}(0)$. That is, we require a large initial nonlinearity.

Recent experimental observations indicate that ${ }^{85} \mathrm{Rb}$ condensates have the most widely tunable interactions via a Feshbach resonance. We refer in particular to experimental results from the JILA group [16,212]. In their results (see [16]) a stable condensate of $10^{4}$ atoms was formed with a variation of the scattering length from zero to $4000 a_{0}$. The associated diluteness factor $n a^{3} \sim 10^{-2}$ indicates such a system has significant interactions but can still be considered to be weakly interacting. We will justify this remark by showing that classical field simulations with this diluteness factor exhibit dynamics that are satisfactorily described by Bogoliubov theory of a weakly interacting Bose gas.

\subsubsection{Simulation parameters}

We employ the parameters from [16], but use a larger atom number of $N_{0}=10^{7}$ while considering the same (peak) number density $N_{0} / V \approx 10^{12} \mathrm{~cm}^{-3}$. We also take the maximum possible initial scattering length to be $a=4000 a_{0}$ at $t=0$.

With these parameters in mind we calculate the dimensionless parameters required for the classical field simulation. From (7.22) and assuming $L_{z} \approx \gamma_{z} V^{1 / 3}$ we can estimate the (dimensionless) initial nonlinear interaction strength is

$$
C_{N L}=\frac{4 \pi a}{\gamma_{z} V^{1 / 3}} N_{0} \approx \frac{1.24 \times 10^{5}}{\gamma_{z}}
$$

The anisotropy parameter should be taken $\gamma_{z}<1$ for the quasi-two dimensional geometry - we do not impose a specific value, but note that we are free to choose a value of the scattering length less than $\sim 4000 a_{0}$. Therefore to meet all the above requirements we select $C_{N L}(\bar{t}=0)=1 \times 10^{5}$ and $N_{0}=10^{7}$ for the simulation results presented in this part of the thesis. While a stable condensate with this atom number has not yet been achieved experimentally, it gives a diluteness factor less than $n a^{3} \sim 10^{-2}$ as found in [16], and so should in principle be possible.

With a large value for $N_{0}$, the thermalisation that can occur in the TWA at large nonlinearities is suppressed. The quasiparticle production demonstrated is then due solely to the effects of $e x$ panding the effective spacetime. Additionally we note that the TWA is valid for short times only 
— this allows us to explore systems undergoing rapid expansion and for which there is appreciable particle production.

Before we embark on the application of the classical field method to expanding universe models (in the next chapter), we characterise the simulation results for a time-independent Hamiltonian (ie. static geometry). We will therefore be able to comment on the validity of the Bogoliubov theory for our range of simulation parameters. We first show how the temperature and elementary excitations of the system can be extracted from the classical field.

\subsection{Estimating the temperature}

We can estimate the temperature of the system from the momentum distribution as follows.

The Bogoliubov Hamiltonian (6.40) represents a system of non-interacting quasiparticles and suggests that the system cannot approach thermal equilibrium. However, the quantum field (via the classical field method) is propagated according to the full Hamiltonian (6.35) which implicitly includes interactions between quasiparticles and therefore the system does indeed thermalise after some time as long as excited modes are occupied (ie. the system is not in the ground state corresponding to the quasiparticle vacuum).

In thermal equilibrium, the mean number of quasiparticles in mode $\mathbf{k}$ is given by the BoseEinstein distribution

$$
N_{\mathbf{k}}=\left\langle\hat{b}_{\mathbf{k}}^{\dagger} \hat{b}_{\mathbf{k}}\right\rangle=\frac{1}{e^{\epsilon_{\mathbf{k}} / k_{B} T}-1}
$$

where $\epsilon_{\mathrm{k}}$ is given by the Bogoliubov dispersion relation (6.46). The Bogoliubov Hamiltonian (6.40) commutes with the quasiparticle number operator so that there are a well defined number of quasiparticles and we can write the mean number of particles in mode $\mathbf{k}$ [213]

$$
\begin{aligned}
N_{\mathbf{k}}^{p} & =\left\langle\hat{a}_{\mathbf{k}}^{\dagger} \hat{a}_{\mathbf{k}}\right\rangle \\
& =u_{\mathbf{k}}^{2} N_{\mathbf{k}}+v_{\mathbf{k}}^{2}\left(N_{-\mathbf{k}}+1\right) \\
& =\frac{N_{\mathbf{k}}+A_{\mathbf{k}}^{2}\left(N_{-\mathbf{k}}+1\right)}{1-A_{\mathbf{k}}^{2}}
\end{aligned}
$$

\section{Equipartition of energy}

Following Davis $[53,214]$, the temperature $T$ can be estimated by assuming equipartition of energy so that

$$
\left\langle N_{\mathbf{k}}\right\rangle_{W}=\frac{k_{B} T}{E_{\mathbf{k}}-\mu}
$$

where $E_{\mathbf{k}}=\epsilon_{\mathbf{k}}+\lambda$ is the energy for each Bogoliubov mode in a condensate with eigenvalue $\lambda$, and $\mu$ is the chemical potential (in the limit of zero temperature we recover $\mu=\lambda$ ). Here $\left\langle N_{\mathbf{k}}\right\rangle_{W}$ refers to the quasiparticle population calculated in the Bogoliubov basis. We can then write [53]

$$
\epsilon_{\mathbf{k}}=k_{B} T\left(\frac{1}{\left\langle N_{\mathbf{k}}\right\rangle_{W}}-\frac{1}{\left\langle N_{0}\right\rangle_{W}}\right)
$$


Defining the dimensionless temperature as $\bar{T}=k_{B} T / \epsilon_{L} N$, where $\epsilon_{L}=m L^{2} / \hbar^{2}$, we then get

$$
\epsilon_{\mathbf{k}}=\bar{T}\left(\frac{N}{\left\langle N_{\mathbf{k}}\right\rangle_{W}}-\frac{N}{\left\langle N_{0}\right\rangle_{W}}\right)=\bar{T} f\left(\left\langle N_{\mathbf{k}}\right\rangle_{W}\right)
$$

Performing a linear fit of $\epsilon_{\mathbf{k}}$ vs $f\left(\left\langle N_{\mathbf{k}}\right\rangle_{W}\right)$, the slope then determines the dimensionless temperature $\bar{T}$.

\section{Bose-Einstein distribution}

To check that equipartition gives accurate results, we can instead perform the same analysis using the Bose-Einstein distribution as our starting point. In this case

$$
\left\langle N_{\mathbf{k}}\right\rangle_{W}=\frac{1}{e^{\left(E_{\mathbf{k}}-\mu\right) / k_{B} T}-1}+\frac{1}{2}
$$

and so

$$
\epsilon_{\mathbf{k}}=\bar{T} \log \left(\frac{\left\langle N_{\mathbf{k}}\right\rangle_{W}+1 / 2}{\left\langle N_{\mathbf{k}}\right\rangle_{W}-1 / 2}\right)-\log \left(\frac{\left\langle N_{0}\right\rangle_{W}+1 / 2}{\left\langle N_{0}\right\rangle_{W}-1 / 2}\right)=\bar{T} f\left(\left\langle N_{\mathbf{k}}\right\rangle_{W}\right)
$$

\subsubsection{Ensemble averaging and ergodicity}

The evolution of the PGPE with initial Wigner noise represents a single trajectory of the system through phase space. To calculate expectation values of any operators we would therefore require an ensemble average over many trajectories. However, this may require a prohibitively large computational time. To avoid this issue, one can appeal to the ergodic hypothesis that states the time average of the system at equilibrium in a single trajectory is the equal to the ensemble average over many trajectories.

\subsection{Power Spectral Density}

It is well known that for a Bose-Einstein condensate the elementary and collective excitations are formally equivalent $[215,216]$. We therefore recover the Bogoliubov dispersion relation (6.46) (or (7.24) in dimensionless units) by calculating the power spectrum of the fluctuations about the homogeneous ground state as follows.

The density fluctuations are

$$
\Delta n(\mathbf{x}, t)=|\psi(\mathbf{x}, t)|^{2}-\left|\psi_{0}(\mathbf{x})\right|^{2}
$$

where $\psi_{0}(\mathbf{x})=1$ is the (normalised) ground state wave-function of the GPE for the homogeneous case. Transforming to momentum space

$$
\Delta n_{\mathbf{k}}(t)=\frac{1}{\sqrt{V}} \int_{V} d \mathbf{x} e^{-i \mathbf{k} \cdot \mathbf{x}} \Delta n(\mathbf{r}, t)
$$




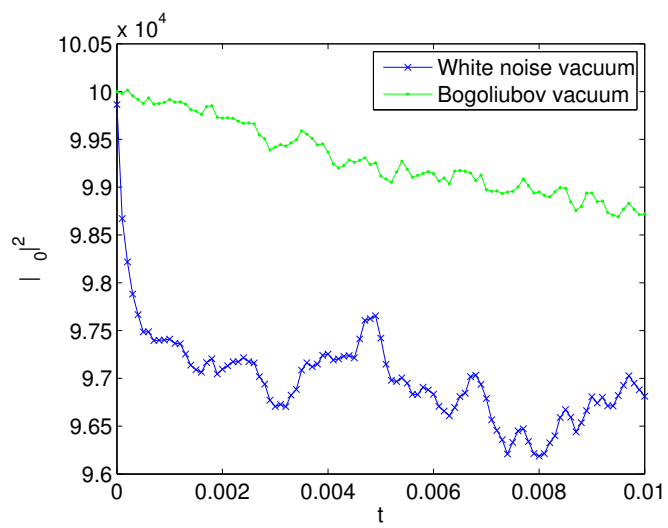

(a) $C_{N L}=1 \times 10^{4}, N_{0}=1 \times 10^{5}$

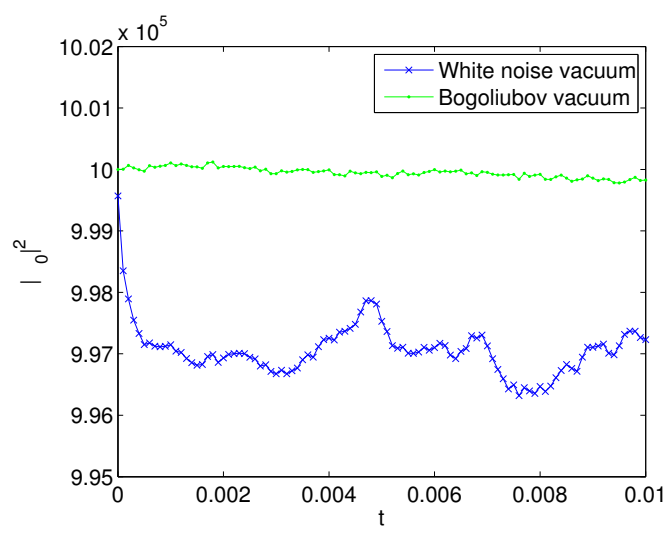

(b) $C_{N L}=1 \times 10^{4}, N_{0}=1 \times 10^{6}$

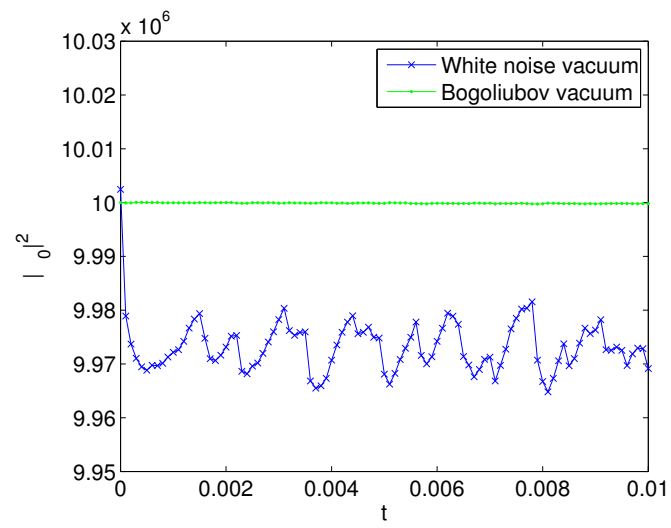

(c) $C_{N L}=1 \times 10^{5}, N_{0}=1 \times 10^{7}$

Figure 7.1: Number of atoms in the condensate vs time for different sets of parameters: each result is for a single trajectory with either a Bogoliubov (quasiparticle) initial vacuum or a white noise (momentum) vacuum. Other parameters are $N_{x}=N_{y}=128$.

The power spectral amplitude is

$$
G(\mathbf{k}, \omega)=\frac{1}{T} \int_{0}^{T} d t e^{-i \omega t} \Delta n_{\mathbf{k}}(t)
$$




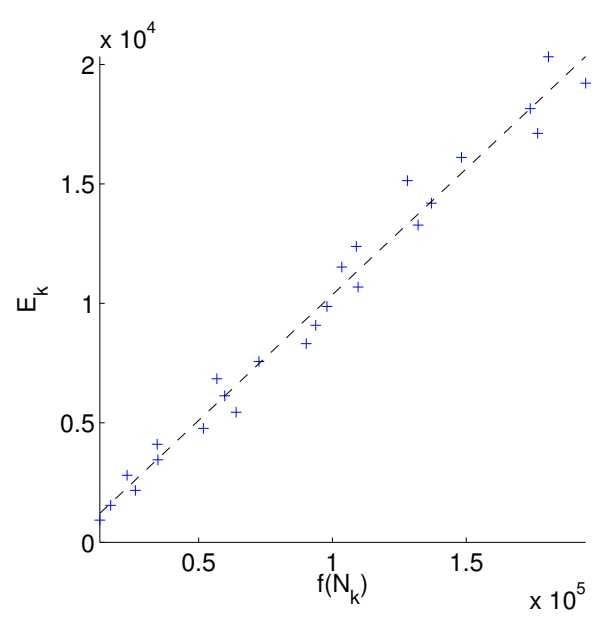

(a) $\bar{T}=0.1053$

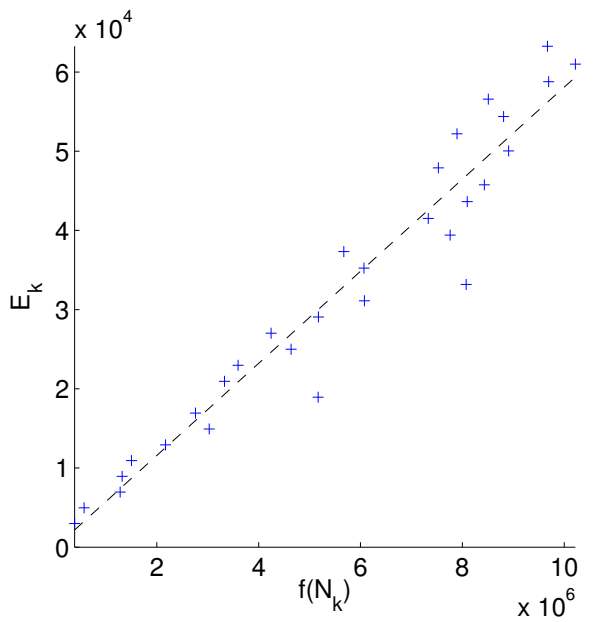

(b) $\bar{T}=0.0058$

Figure 7.2: Equipartition of energy: linear fit of (7.30) to give temperature $\bar{T}$. The initial state includes vacuum fluctuations for a white noise vacuum (ie. 1/2 particle per momentum mode). Ergodicity has been assumed and the results are therefore time averaged over the final 50 simulation time points. Parameters are: (a) $C_{N L}=10^{4}, N_{0}=10^{5}$; (b) $C_{N L}=10^{5}, N_{0}=10^{7}$.

with corresponding power spectral density given by

$$
P(\mathbf{k}, \omega)=G^{*}(\mathbf{k}, \omega) G(\mathbf{k}, \omega)
$$

To find the spectrum for $k=|\mathbf{k}|$, we further average over angle in momentum space by resampling the distribution in polar coordinates to give $P(k, \omega)$.

\subsection{Results for Homogeneous BEC}

We present the results of classical field simulations for the case of a homogeneous Bose-Einstein condensate where all external parameters are time-independent. Our motivation here is to establish that the quasiparticle vacuum represents an acceptable ground state for the range of simulation parameters we utilise in the expanding universe model simulations presented in the next chapter.

\subsubsection{Condensate depletion}

Clearly if the initial state for the classical field simulation is the correct ground state, the system will evolve away from this state towards equilibrium (via interactions between quasiparticle modes) - this can lead to depletion of the condensate mode. Figures 7.1(a)-(c) show the condensate density for three different sets of parameters. In particular, Figure 7.1(a) has $C_{N L}=1 \times 10^{4}$, $N_{0}=1 \times 10^{5}$, Figure 7.1(b) has $C_{N L}=1 \times 10^{4}, N_{0}=1 \times 10^{6}$, and Figure 7.1(c) has $C_{N L}=1 \times 10^{5}, N_{0}=1 \times 10^{7}$. In all cases, the initial state with a white noise vacuum leads to further depletion of the condensate, whereas the effect with the Bogoliubov quasiparticle vacuum is minimal. Moreover, for the the case of $C_{N L}=1 \times 10^{5}$ and $N_{0}=1 \times 10^{7}$ the Bogoliubov 


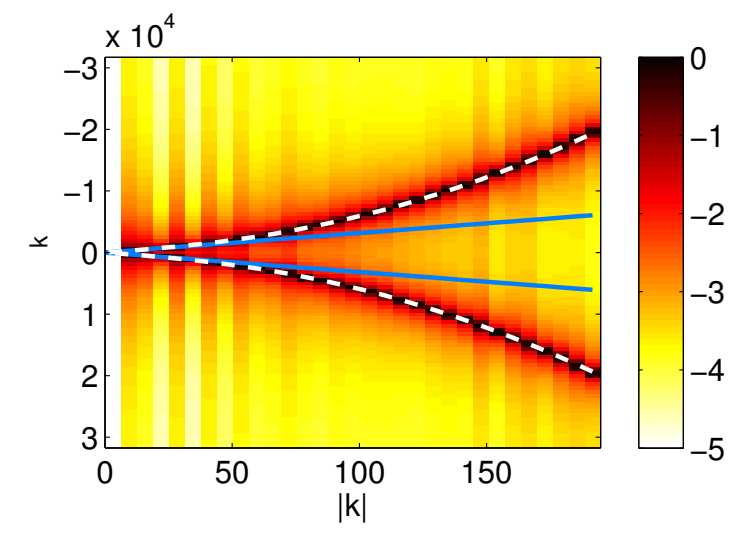

(a) $C_{N L}=1 \times 10^{3}$

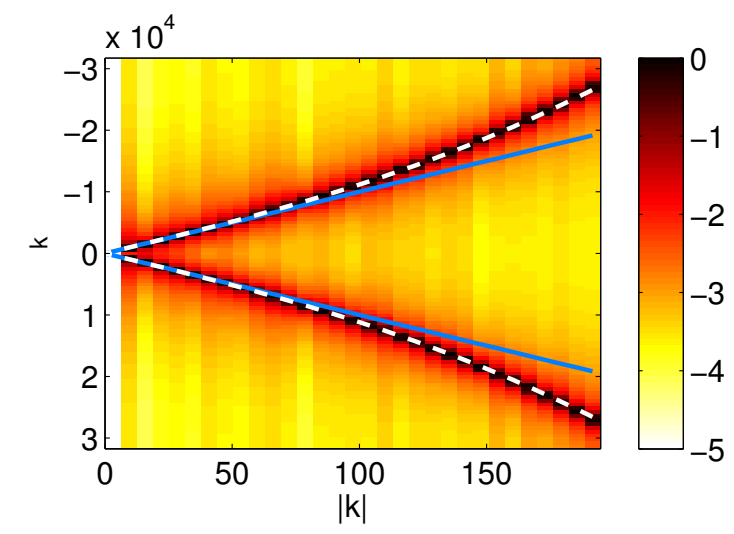

(b) $C_{N L}=1 \times 10^{4}$

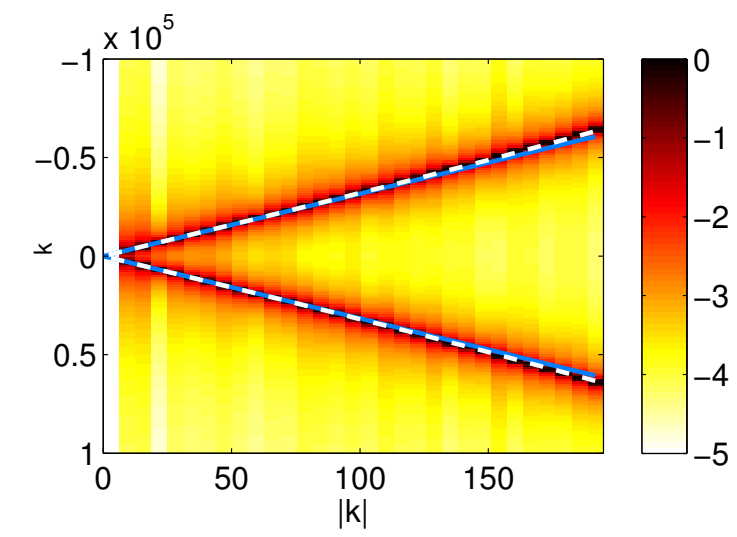

(c) $C_{N L}=1 \times 10^{5}$

Figure 7.3: Calculated dispersion relation from (7.36). The numerical results are given by an density plot of the $\log$ of the power spectrum (renormalised so that the peak value is unity) with the darker regions indicating higher density. Results are ensemble averaged over 10 runs with parameters $N_{0}=10^{7}, N_{x}=N_{y}=128$. For comparison, also shown is the analytic result (white dashed curve) from the Bogoliubov theory (7.24), and the phonon branch of the spectrum $\omega \sim c k$ (blue solid curve). 
quasiparticle vacuum leads to negligible depletion of the condensate. We further note that the simulation time of $t=1 \times 10^{-2}$ used here exceeds the simulation time for most of the results presented in the next chapter (the exception is the tanh expansion model).

\subsubsection{Temperature estimates for white noise vacuum}

We have seen that when the initial state includes Gaussian white noise the system undergoes a transient thermalisation. This reflects the fact that this initial state does not correspond to the quasiparticle vacuum for the interacting system. We can calculate the temperature of the system using the equipartition of energy as outlined in section 7.5. We present two examples to demonstrate the efficacy of the procedure. For these results we utilise the ergodic hypothesis and so time average over the last 50 simulation time points to give the quasiparticle distribution. Figure 7.2 shows the linear fit of (7.30) to give the dimensionless temperature $\bar{T}$. The case of $C_{N L}=10^{4}$ and $N_{0}=10^{5}$ leads to a larger temperature compared to the case of $C_{N L}=10^{5}$ and $N_{0}=10^{7}$, which is consistent with the corresponding depletion of the condensate mode.

\subsubsection{Elementary excitations}

We plot the intensity of the power spectrum (7.36) in Figure 7.3 for three different nonlinearities, and with the analytic expression for the Bogoliubov dispersion relation given by (7.24) - the agreement is excellent and we can surmise that the Bogoliubov theory is accurate for the regime in which we are working. The solid blue curve represents the phonon branch of the spectrum with $\omega \sim c k$, which dominates for the highest value of the nonlinearity as per Figure 7.3(c). In contrast for the lowest value of the nonlinearity shown in Figure 7.3(a) the dispersion is approximately quadratic as the single-particle energy dominates over the interaction energy.

The recovery of the Bogoliubov dispersion relation from the power spectral density reflects the fact that the collective and elementary excitations are equivalent in a Bose-condensed system [216].

\subsection{Conclusions}

We have introduced the classical field method based on the truncated Wigner approximation; this tool is used to simulate the FRW expanding universe models introduced in chapter 6, with results presented in the next chapter. The choice of parameters $C_{N L}=10^{5}$ and $N_{0}=10^{7}$ for these simulations has been justified on both physical and technical grounds. In particular, the initial state that is constructed using a Bogoliubov quasiparticle vacuum is suitable for these simulations as the effects of thermalisation are minimal - this is an important consideration since quasiparticle production in an expanding universe model can be a small effect, which otherwise might be obscured by a thermal component arising from the initial state. 


\section{Chapter 8}

\section{Expanding Universe simulations}

\subsection{Introduction}

We present the numerical results of classical field simulations based on the TWA for the expansion scenarios outlined in Section 6.5 - namely the de Sitter expansion, tanh and cyclic expansion scenarios, with the sudden transition as a limit of an infinitely fast de Sitter or tanh expansion. The results are compared to the analytic predictions in the acoustic approximation, and also to the sudden transition prediction that includes the nonlinear dispersion of the modes (see equation $6.95)$.

\subsection{Quasiparticle production results}

In the results shown, we have calculated the quasiparticle populations for each mode as a function of time; this was accomplished by projecting from the single particle basis to the Bogoliubov basis using the expression (7.18), and using the nonlinearity $C_{N L}(\bar{t})$. Thus the basis for counting quasiparticles corresponds to projecting into the instantaneous Minkowski vacuum at each time.

\subsection{1 de Sitter universe}

For an expansion corresponding to the de Sitter universe, the scaling function $b(t)$ takes the form (6.80). Figure 8.1 shows the results for an expansion of $X=2000$ and four different rates of expansion $t_{s}=1 \times 10^{-5}, 5 \times 10^{-5}, 1 \times 10^{-4}$ and $1 \times 10^{-3}$.

For comparison, the sudden transition result for $X=2000$ from (6.96) is shown at the final time by the red dashed curve. This gives the upper limit on the permissible particle production in each mode.

Also shown is the time $t_{c}$ when each mode crosses from phonon to particle-like behaviour due to the expansion of the universe, as determined by $k^{2} / 2=C_{N L}\left(t_{c}\right)$ - this is indicated by the blue points on each plot.

An intuitive picture of the effect of quasiparticle production is demonstrated by Figure 8.2, which gives the field density at the initial and final times for the case of $t_{s}=1 \times 10^{-5}$. The small scale fluctuations given by the initial quasiparticle (Bogoliubov) vacuum are amplified to a larger 


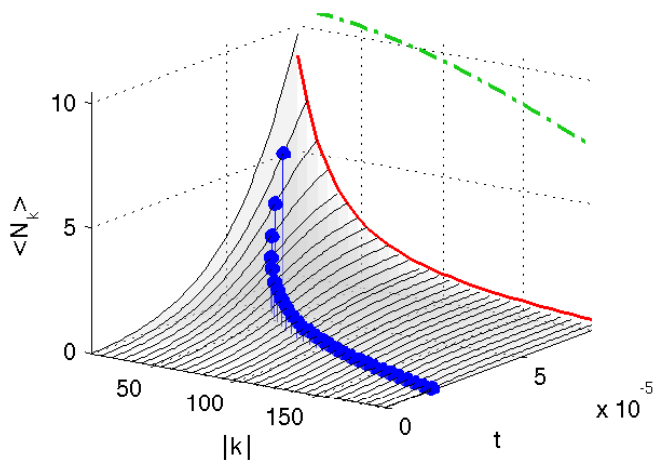

(a) $t_{s}=1 \times 10^{-5}$

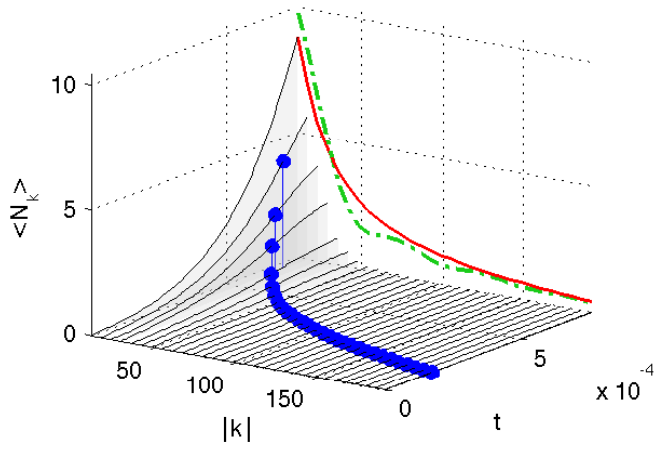

(c) $t_{s}=1 \times 10^{-4}$

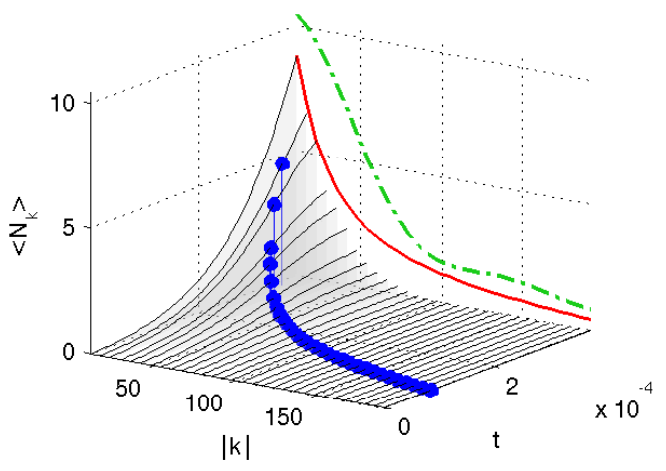

(b) $t_{s}=5 \times 10^{-5}$

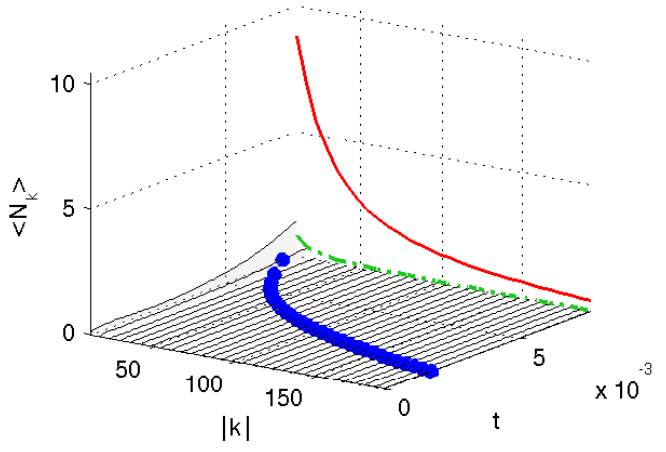

(d) $t_{s}=1 \times 10^{-3}$

Figure 8.1: de Sitter expansion: time dependence of Bogoliubov mode populations. Parameters are $C_{N L}(\bar{t}=0)=1 \times 10^{5}, N_{0}=10^{7}$ and $X=2 \times 10^{3}$. The blue points on each curve show where each mode crosses over from phonon to free-particle behaviour (as defined by $\bar{k}_{c}^{2} / 2=C_{N L}$ ). The green dashed curve shows the analytic prediction in the acoustic approximation from (6.68). The red solid curve shows the analytic prediction for a sudden transition from (6.95).

scale after expansion has occurred.

\subsubsection{Modified de Sitter expansion}

In an actual experiment, it is unfeasible to implement the de Sitter expansion we have described previously in section 6.5.3. A sudden switch on of the expansion would require a discontinuous variation in the external magnetic field for an initially prepared condensate, which would lead to higher order scattering processes. In this case the effective field theory that our model depends on becomes invalid. Moreover, any variation in an external parameter necessarily requires a finite albeit small - time.

To address this issue we consider a lead in time $t_{I}$ where the nonlinearity smoothly varies from a constant initial value $C_{N L}(0)$ to the de Sitter expansion starting at $t=t_{I}$ and lasting for a time $t_{I I}$. Moreover we add a lead out time $t_{I I I}$ where the scaling function varies continuously from the end of the de Sitter expansion at $t=t_{I}+t_{I I}$ to a constant value at the final time $t_{f}=t_{I}+t_{I I}+t_{I I I}$. Such a modified de Sitter expansion could be implemented with the piecewise scaling function: 

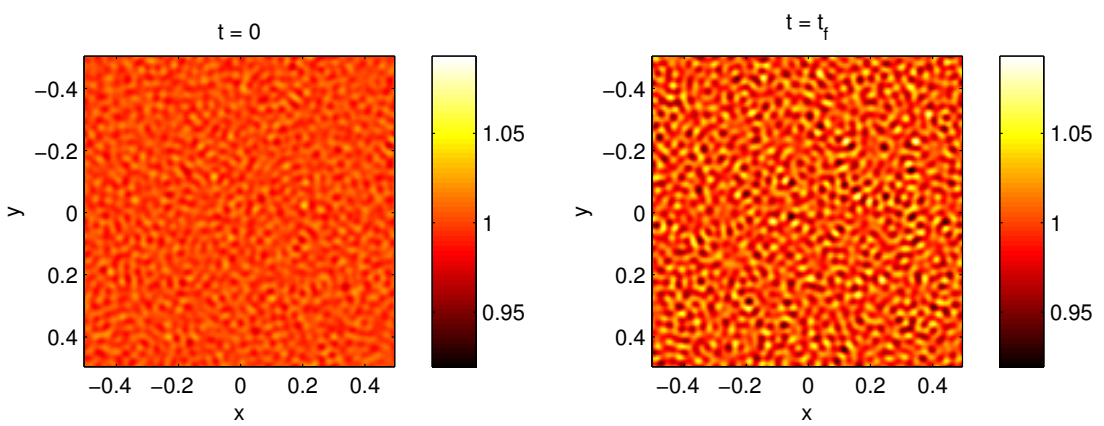

Figure 8.2: de Sitter model: density plot for renormalised wavefunction at beginning $(t=0)$ and at end $\left(t=t_{f}\right)$ of expansion. Parameters are $t_{s}=1 \times 10^{-5}, C_{N L}(t=0)=1 \times 10^{5}$, and $N_{0}=10^{7}$.

$t \leq t_{I}$ (parabolic):

$$
b(t)=\frac{-1}{\left(2 t_{s}+t_{I}\right) t_{I}} t^{2}+1
$$

$t_{I} \leq t \leq t_{I}+t_{I I}$ (de Sitter):

$$
b(t)=\frac{2 t_{s}}{2 t_{s}+t_{I}} e^{\left(t_{I}-t\right) / t_{s}}
$$

$t_{I}+t_{I I} \leq t \leq t_{I}+t_{I I}+t_{I I I}$ (parabolic):

$$
b(t)=\frac{e^{-t_{I I} / t_{s}}}{2 t_{s}+t_{I}}\left[\frac{1}{t_{I I I}}\left(t-t_{I}-t_{I I}\right)^{2}-2\left(t-t_{I}-t_{I I}\right)+2 t_{s}\right]
$$

The definition of the expansion $X$ gives

$$
b\left(t_{I}+t_{I I}+t_{I I I}\right)=\frac{1}{X}
$$

or from $(8.3)$

$$
\frac{2 t_{s}-t_{I I I}}{2 t_{s}+t_{I}} e^{-t_{I I} / t_{s}}=\frac{1}{X}
$$

We consider two cases for a modified de Sitter expansion. For convenience we choose the lead-in and lead-out times such that $t_{I}=t_{I I I}=\gamma t_{s}$.

Case (i) $t_{s}$ fixed: In this case we fix $t_{s}$ so that

$$
t_{f}=t_{s}\left[\log \left(\frac{2-\gamma}{2+\gamma} X\right)+2 \gamma\right]
$$

Case (ii) $t_{f}$ fixed: In this case we fix $t_{f}$ so that

$$
t_{s}=t_{f}\left[\log \left(\frac{2-\gamma}{2+\gamma} X\right)+2 \gamma\right]^{-1}
$$




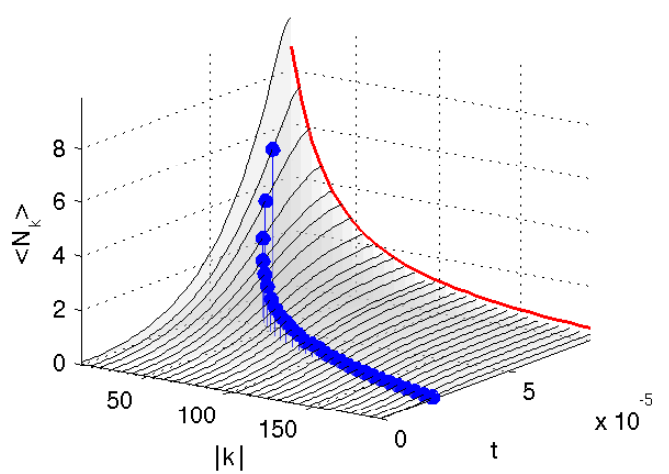

(a) $t_{s}=1 \times 10^{-5}$

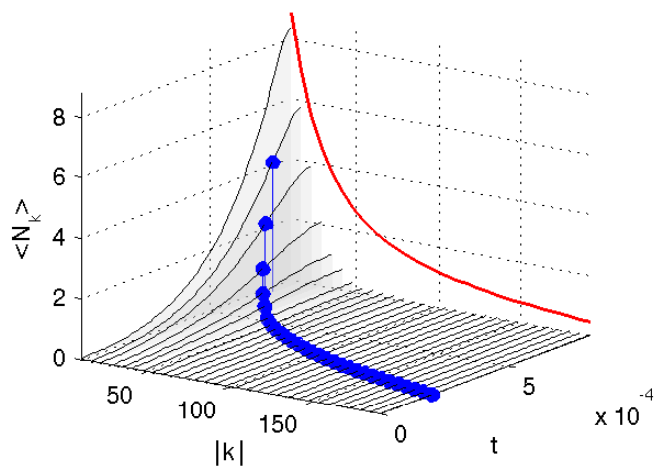

(c) $t_{s}=1 \times 10^{-4}$

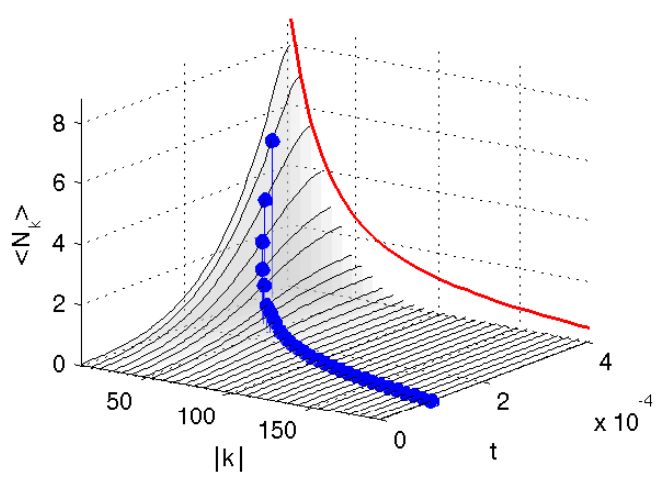

(b) $t_{s}=5 \times 10^{-5}$

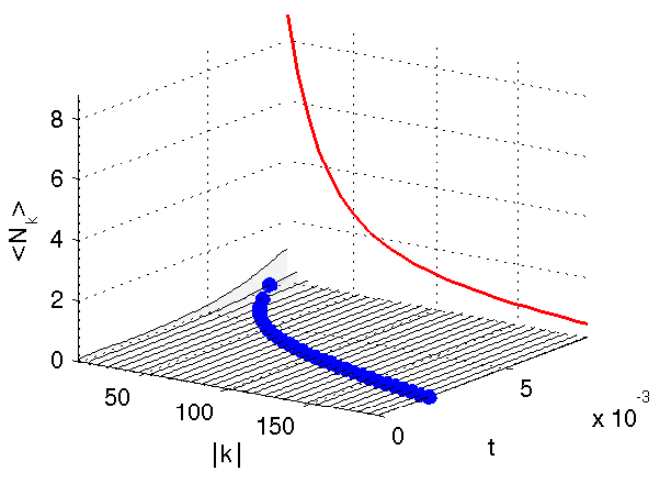

(d) $t_{s}=1 \times 10^{-3}$

Figure 8.3: Modified de Sitter expansion: time dependence of Bogoliubov mode populations. Parameters are $C_{N L}(\bar{t}=0)=1 \times 10^{5}, N_{0}=10^{7}$ and $X=2 \times 10^{3}$. The lead in and lead out times are given by $t_{I}=t_{I I I}=\frac{1}{2} t_{s}$. The blue points on each curve show where each mode crosses over from phonon to free-particle behaviour (as defined by $\bar{k}_{c}^{2} / 2=C_{N L}$ ). The red solid curve shows the analytic prediction for a sudden transition from (6.95).

Figure 8.3 shows results for a modified de Sitter expansion with the scaling function given by (8.6) with $\gamma=1 / 2$ and all other parameters the same as the de Sitter results given in Figure 8.1. The results are nearly identical to those given in Figure 8.1 indicating that the modified scale factor does not invalidate the de Sitter results. The slight reduction of particle production in the modified de Sitter case can be attributed to the slightly slower expansion that results from using (8.6); that is, $t_{f} \approx 8.1 t_{s}$ for the modified de Sitter expansion compared with $t_{f} \approx 7.6 t_{s}$ for the standard de Sitter expansion (both cases have the same expansion $X=2 \times 10^{3}$ ).

\subsection{3 tanh Expansion}

In this case scaling function $b(t)$ is given by the parametric curve in $\tilde{t}$ by equations (6.77) and (6.78). The form of $b(t)$ tends to exhibit a rapid early change followed by a long tail as it approaches its final value $b\left(t_{f}\right)$. Due to the computational expense in running simulations for long times, we took the final simulation time as $\tilde{t}_{f}=\tilde{t}_{s} \tanh ^{-1}(0.999)$. The final value of the scal- 


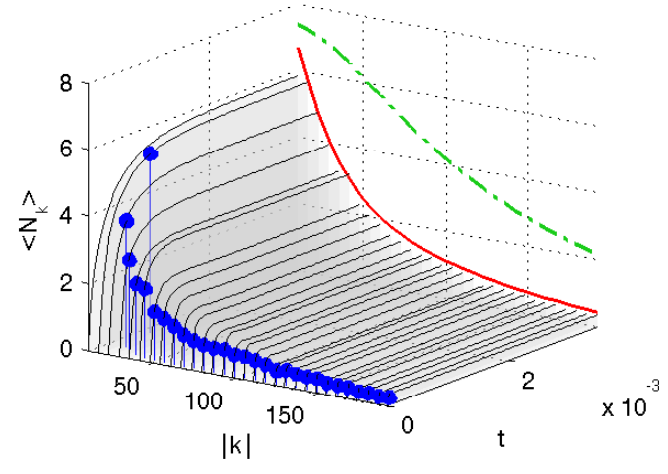

(a) $\tilde{t}_{s}=1 \times 10^{-1}$

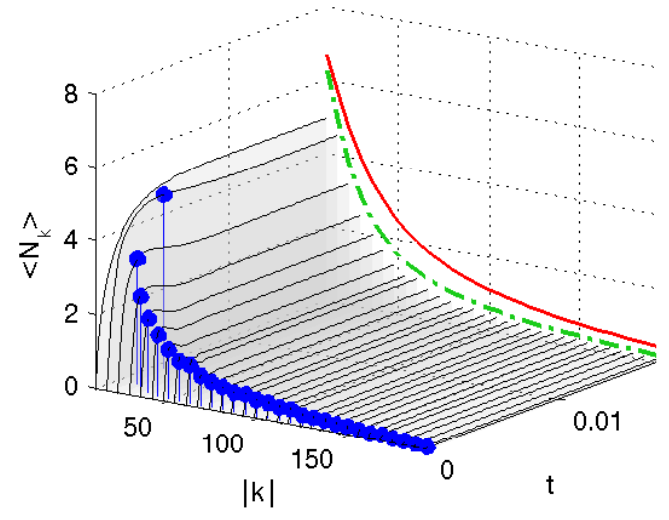

(b) $\tilde{t}_{s}=5 \times 10^{-1}$

Figure 8.4: tanh expansion: time dependence of Bogoliubov mode populations. Parameters are $C_{N L}(\bar{t}=0)=1 \times 10^{5}, N_{0}=10^{7}$ and $X=10^{3}$. The blue points on each curve show where each mode crosses over from phonon to free-particle behaviour (as defined by $\bar{k}_{c}^{2} / 2=C_{N L}$ ). The green dashed curve shows the analytic prediction in the acoustic approximation from (6.79). The red solid curve shows the analytic prediction for a sudden transition from (6.95).

ing function $b\left(t_{f}\right)$ then reached $\approx 99.9 \%$ of its target value $1 / X$. This minor discrepancy had a negligible effect on the results shown.

Figure 8.4 shows the results for an expansion of $X=1000$ and two different rates of expansion $\tilde{t}_{s}=0.1$ and 0.5 . Similarly to the de Sitter expansion results, the sudden result for $X=1000$ is shown by the red solid curve and the phonon to particle-like crossover is indicated by the blue points. Moreover, the green dotted curve shows the particle production for each mode according to the prediction for the acoustic approximation given by (6.79).

\subsubsection{Cyclic universe}

In this case, we consider the oscillating scaling function given by (6.97). In particular, we consider two subcases: a single cycle $(m=1)$, which corresponds to a single expansion and contraction for the effective spacetime; and multiple cycles $(m>1)$, which correspond to $m$ expansion and contractions. The peak wave-vector $k_{\text {resonance }}$ for parametric resonance from (6.98) is shown by the position of the red solid line in each case.

\section{Single cycle $(m=1)$}

We further consider two specific sets of parameters: (i) $m=1, X=2000$ and $t_{s}=1 \times 10^{-5}$; $m=1, X=2000$ and $t_{s}=1 \times 10^{-4}$. The corresponding simulation results are given by Figures 8.5(a) and (b). In both cases there is a transient phase where there is dramatic quasiparticle production in the time-dependent Bogoliubov basis; however, the net quasiparticle production at the end of the cycle $\left(t=t_{f}\right)$ is small in both cases, and in particular is very close to zero for the faster cycle in Figure 8.5(a). Note that for $t_{s}=1 \times 10^{-5}$ the resonance condition occurs for a 


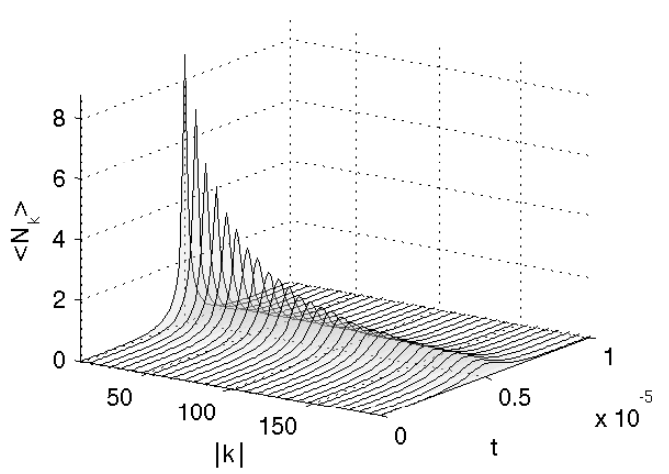

(a) $t_{s}=1 \times 10^{-5}$

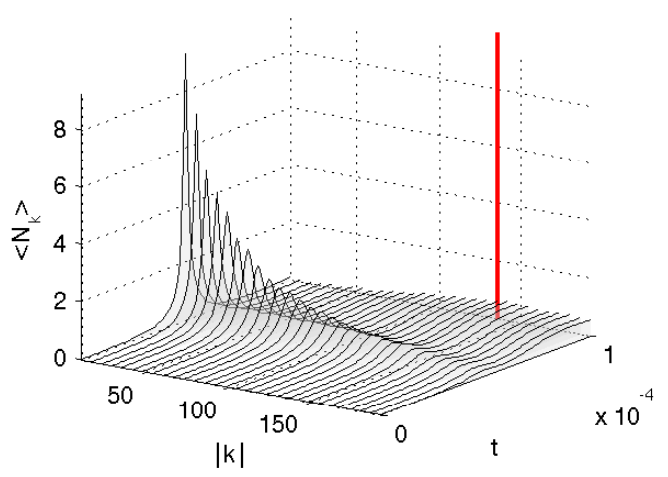

(b) $t_{s}=1 \times 10^{-4}$

Figure 8.5: Cyclic universe: Nonlinearity vs time (left) and time dependence of Bogoliubov mode populations (right) for a single cycle $(m=1)$ and for two different periods $t_{s}$. Parameters are $C_{N L}(\bar{t}=0)=1 \times 10^{5}, N_{0}=10^{7}$ for both cases. The red solid line shows the position of the peak wave-vector for parametric resonance from the analytic prediction (6.98).

wave-vector larger than the cut-off for the projector (ie. $k_{\text {resonance }}>k_{\text {cut-off }}$ ).

\section{Multiple cycles $(m>1)$}

The excitation of the field due to parametric resonance is much greater when there are multiple cycles of the scaling factor, and we can therefore consider a smaller driving amplitude $X$. In particular, we consider three specific sets of parameters for this subcase: (i) $m=5, X=100$ and $t_{s}=1 \times 10^{-4}$; (ii) $m=10, X=2$ and $t_{s}=1 \times 10^{-4}$; and (iii) $m=10, X=2$ and $t_{s}=2 \times 10^{-4}$. The corresponding simulation results are given by Figures 8.6(a)-(f). Clearly the mode population is peaked near the resonance condition.

For the two cases with $m=10$ and $X=2$, the field density at the initial and final times are given by Figures 8.7 and 8.8 ; the parametric resonance leads to the amplification of large scale fluctuations on the length scale that corresponds to the peak mode frequency. This can be clearly seen as the density fluctuations for the final simulation time in Figure 8.8 are on a longer scale than those shown in 8.7 .

\subsection{Discussion}

\subsubsection{Inflationary universe models}

The healing length increases as the expansion proceeds; a mode $k$ that starts as phononic $(k<$ $1 / \xi(0))$ will at a later time $t_{c}$ cross-over to a particle-like regime $\left(k \sim 1 / \xi\left(t_{c}\right)\right)$. According to the discussion of section 6.3 we expect particle production to be dominant before this time, the mode populations becoming fixed after this time. This prediction is bourne out in the results shown in Figures 8.1 and 8.4 .

Moreover, as is clear in these results, the sudden transition prediction given by (6.96) sets an 


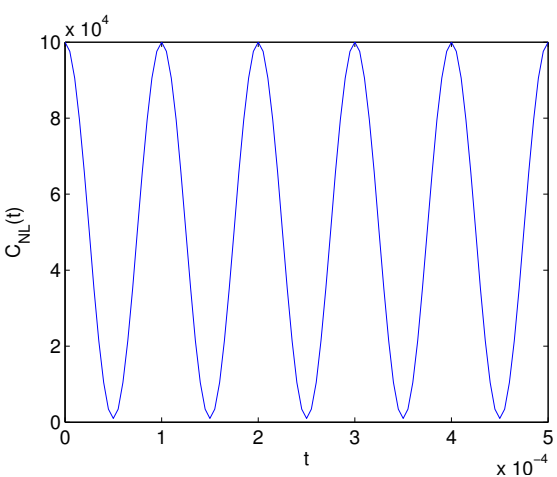

(a) $m=5, X=100, t_{s}=1 \times 10^{-4}$

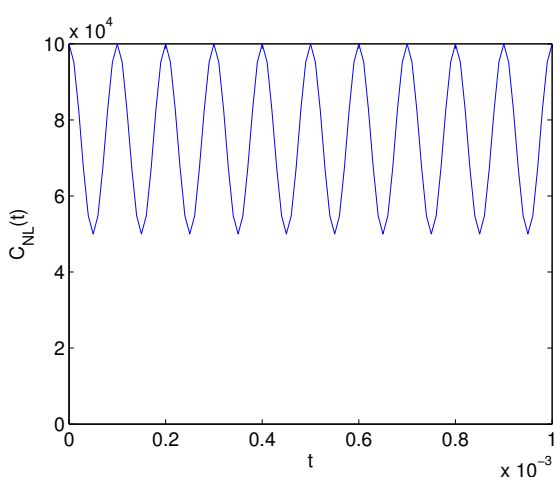

(c) $m=10, X=2, t_{s}=1 \times 10^{-4}$

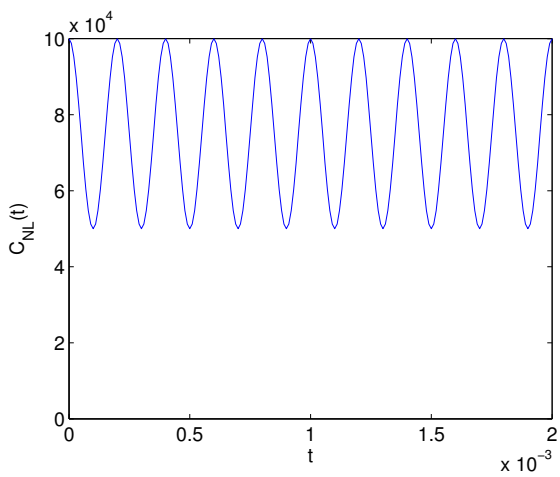

(e) $m=10, X=2, t_{s}=2 \times 10^{-4}$

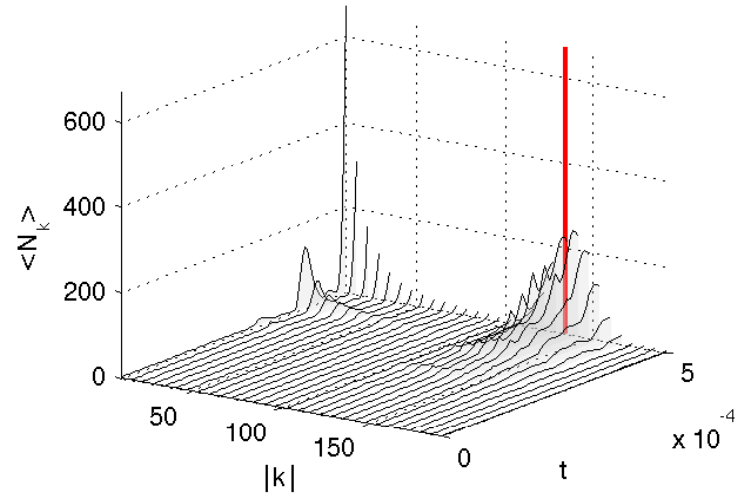

(b) $m=5, X=100, t_{s}=1 \times 10^{-4}$

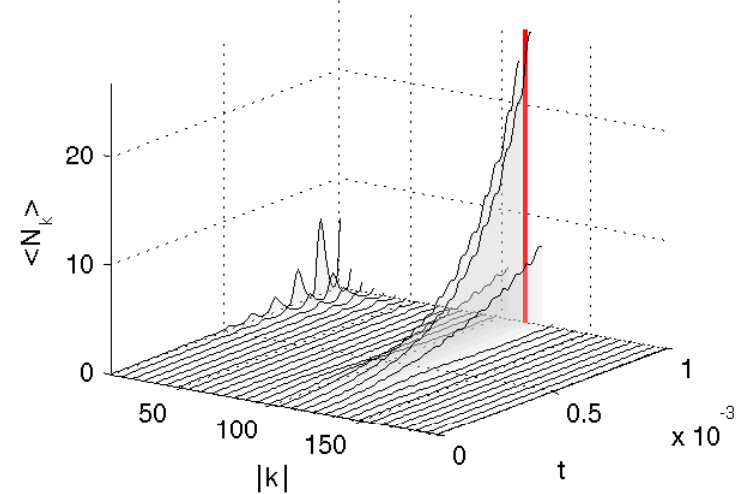

(d) $m=10, X=2, t_{s}=1 \times 10^{-4}$

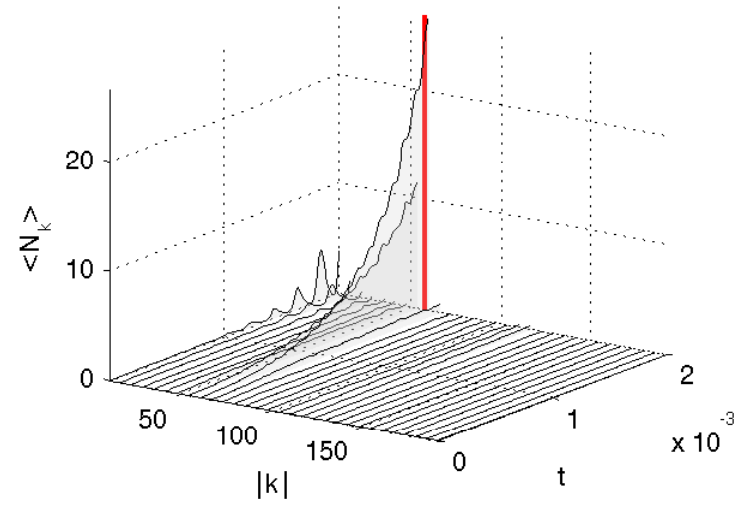

(f) $m=10, X=2, t_{s}=2 \times 10^{-4}$

Figure 8.6: Cyclic universe: Nonlinearity vs time (left) and time dependence of Bogoliubov mode populations (right) for three different scenarios. Parameters are $C_{N L}(\bar{t}=0)=1 \times 10^{5}, N_{0}=$ $10^{7}$ for all cases. The red solid line shows the position of the peak wave-vector for parametric resonance from the analytic prediction (6.98).

upper limit on the particle production in each mode. A sudden transition corresponds to $t_{s} \rightarrow 0$ in the de Sitter expansion case, or $\tilde{t}_{s} \rightarrow 0$ in the tanh expansion case; in this limit the classical 

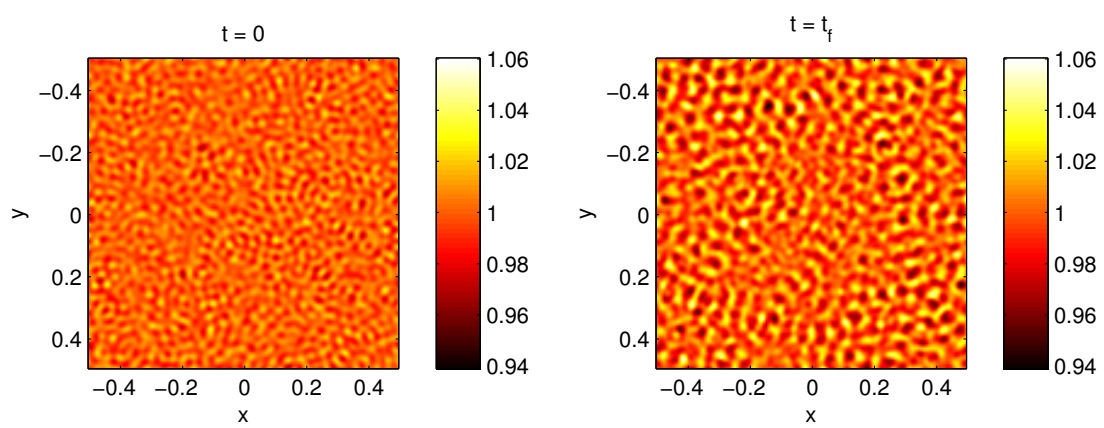

Figure 8.7: Cyclic model: Density plot for renormalised wavefunction at beginning $(t=0)$ and at end $\left(t=t_{f}\right)$ of expansion. Parameters are $m=10, X=2, t_{s}=1 \times 10^{-4}, C_{N L}(t=0)=1 \times 10^{5}$, and $N_{0}=10^{7}$.
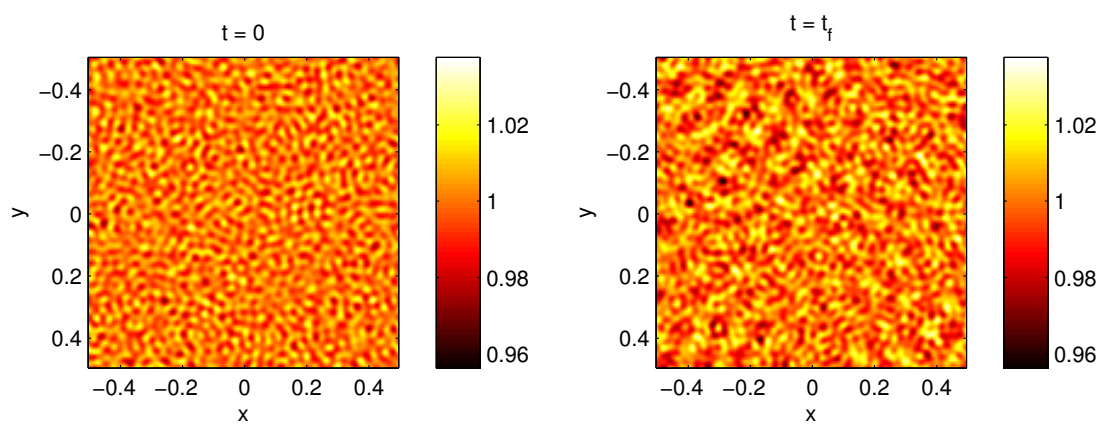

Figure 8.8: Cyclic model: Density plot for renormalised wavefunction at beginning $(t=0)$ and at end $\left(t=t_{f}\right)$ of expansion. Parameters are $m=10, X=2, t_{s}=2 \times 10^{-4}, C_{N L}(t=0)=1 \times 10^{5}$, and $N_{0}=10^{7}$.

field does not evolve, but the Bogoliubov basis that diagonalises the Hamiltonian changes. The results clearly indicate that the particle production approaches the sudden transition prediction in both scenarios when the expansion rate is largest - see Figures 8.1(a) and 8.4(a). The discrepancy for small $|\mathbf{k}|$ in the tanh expansion case is due to nonlinear interactions, which are neglected in the free field theory of Section 6.3. We elaborate on this point below.

\subsubsection{Thermal equilibrium and the adiabatic regime}

There are two factors which can lead to a thermal spectrum for the occupation numbers of quasiparticles. Firstly, if the expansion is adiabatic, the particle production leads to a thermal spectrum [121]. This is the case even when the underlying theory is for a free-field as in section 6.2. Secondly and more generally, the CFM is based on the field dynamics for the Hamiltonian (6.35) which implicitly includes higher order terms not present in the approximate Bogoliubov Hamiltonian (6.40); therefore the Bogoliubov modes are interacting — albeit weakly — and even in the absence of damping the system will eventually approach thermal equilibrium due to ergodicity.

In the CFM the temperature for a weakly interacting system can be estimated by assuming 


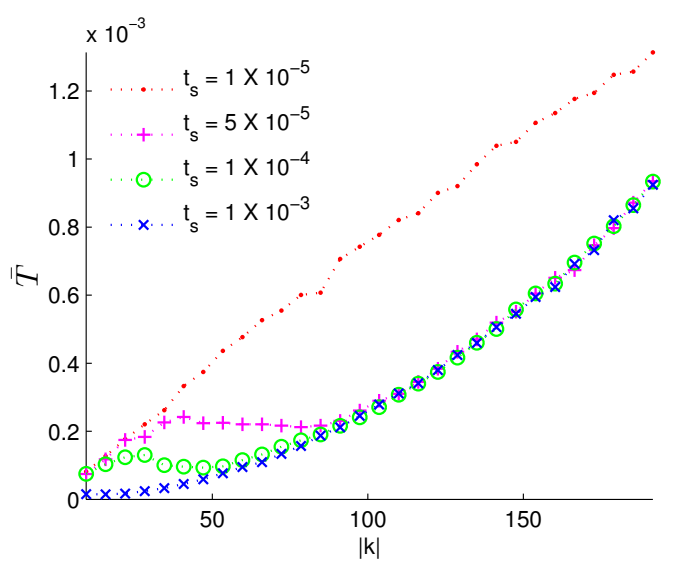

Figure 8.9: Equipartition of energy at the final time for de Sitter expansion with four different expansion rates. Parameters are $C_{N L}(t=0)=1 \times 10^{5}, N_{0}=10^{7}$ and $X=2 \times 10^{3}$.

equipartition of energy. Following Davis et al. [53], this gives:

$$
\left\langle N_{\mathbf{k}}\right\rangle_{W}=\frac{k_{B} T}{E_{\mathbf{k}}-\mu}
$$

where $E_{\mathbf{k}}=\epsilon_{\mathbf{k}}+\lambda$ is the energy for each Bogoliubov mode in a condensate with eigenvalue $\lambda$, and $\mu$ is the chemical potential. Here $\left\langle N_{\mathbf{k}}\right\rangle_{W}$ refers to the quasiparticle population calculated in the Bogoliubov basis. We can then write

$$
\epsilon_{\mathbf{k}}=k_{B} T\left(\frac{1}{\left\langle N_{\mathbf{k}}\right\rangle_{W}}-\frac{1}{\left\langle N_{0}\right\rangle_{W}}\right)
$$

Defining the dimensionless temperature as $\bar{T}=k_{B} T / \epsilon_{L} N$, where $\epsilon_{L}=m L^{2} / \hbar^{2}$, and rearranging (8.9) gives

$$
\bar{T}=\bar{\epsilon}_{\mathbf{k}}\left(\frac{N}{\left\langle N_{\mathbf{k}}\right\rangle_{W}}-\frac{N}{\left\langle N_{0}\right\rangle_{W}}\right)^{-1}
$$

That is, if the system is in thermal equilibrium the function $\bar{T}(|\mathbf{k}|)$ should be constant.

In Figure 8.9 we plot this function for the de Sitter expansion results with $t_{s}=1 \times 10^{-5}, 5 \times$ $10^{-5}, 1 \times 10^{-4}$ and $1 \times 10^{-5}$, and at the final time in each case for $X=2000$. For the fastest expansion with $t_{s}=1 \times 10^{-5}, \bar{T}$ is approximately linear, indicating the system is not in thermal equilibrium. This is expected since the fastest expansion rate approaches the sudden expansion case, for which the particle production is not thermal as we have previously seen for the acoustic approximation (see Figure 6.3). For the slowest expansion with $t_{s}=1 \times 10^{-3}$, there is negligible particle production so that the mode populations are fixed at the initial value of half a particle per mode for the classical field. In this case it follows from (8.10) that $\bar{T} \sim \bar{\epsilon}_{\mathbf{k}}$ as evident in the plot.

In contrast, we note for the two intermediate expansion rates $\left(t_{s}=5 \times 10^{-5}\right.$ and $\left.1 \times 10^{-4}\right)$, $\bar{T}$ is relatively flat for small $|\mathbf{k}|$ which corresponds to the regions in Figure 8.1 where the particle production is most significant. In these cases, the slower expansions result in an approximately 
thermal spectrum for the phononic modes, which is consistent for adiabatic expansion in the freefield theory. For the larger $|\mathbf{k}|$ modes, no particle production occurs, and the mode populations are frozen at the initial value of half a particle per mode in the classical field. If the field was further evolved at the final nonlinearity $C_{N L}(t=0) / X$, the system should eventually reach thermal equilibrium via ergodicity. This effect is evident in the tanh expansion results (Figure 8.4) where the nonlinearity $C_{N L}$ asymptotically approaches a non-zero final value. In particular, the nonlinear mode mixing accounts for the discrepancy between the analytic predictions for the free-field theory and the particle production in the low $|\mathbf{k}|$ modes. This effect is more pronounced in Figure 8.4(b) where the system evolves for a much longer time.

\subsubsection{Cyclic universe model}

The mode spectrum that results from the implementation of a cyclic universe model is markedly different to the case of inflationary expansion (ie. de Sitter, tanh or sudden expansions). Specifically, there is a non-zero wave-vector at which the mode population peaks, which is given by the condition for parametric resonance as discussed in section 6.6.2. The results for a cyclic universe are given for a single cycle by Figures 8.5(a) and (b), and for multiple cycles by Figures 8.6(a)-(f).

For the subcase of a single cycle $(m=1)$ there are two observable effects:

- A peak in quasiparticle number midway through the cycle, which corresponds to the usual notion of particle production due to expansion as in the inflationary models. This is an artifact of projecting the quasiparticle number into the time-dependent Bogoliubov basis with nonlinearity $U=U_{0} / X$.

- The net quasiparticle number at the end of the cycle is determined by projecting into the Bogoliubov basis with nonlinearity $U\left(t_{f}\right)=U(0)$. That is, the effective spacetime is the same at the start and end of the cycle (providing quasiparticle production does not lead to appreciable depletion of the condensate). Any quasiparticle production is then attributed to parametric excitation, which peaks for the wave-vector corresponding to the resonance condition (6.98). For the cycle with a longer period $\left(t_{s}=1 \times 10^{-4}\right)$, shown in Figure 8.5(b), the resonant wave-vector is within the projected mode-space (ie. $k_{\text {resonance }}<k_{\text {cut-off }}$ ) and there is non-zero quasiparticle production at the end of the cycle. In contrast, for the cycle of shorter period $\left(t_{s}=1 \times 10^{-4}\right)$, shown in Figure 8.5(a), the resonant condition occurs for modes outside the projected mode-space (ie. $k_{\text {resonance }}>k_{\text {cut-off }}$ ) and there is negligible quasiparticle production for the system modes.

Quasiparticle production is more dramatic for the case of multiple cycles as we can see from the results in Figures 8.6(a)-(f). In particular, referring to the discussion in section 6.6.2, for the first case with $m=5$ and $X=100$, the parametric resonance leads to a broad peak which can be attributed to the large value of $X$. By contrast, for the second case with $m=10$ and $X=2$ the peak is narrower due to the smaller value of $X$. A thermal component is also evident in both cases for low momenta modes, due to interactions between the quasiparticle modes. This component is expected to grow with longer evolution times as the modes continue to interact (noting that the magnitude of $C_{N L}$ is significant throughout the evolution). 


\subsection{Conclusions}

In summary, we have run classical field simulations for analogue models of inflationary cosmology (de Sitter and tanh expansions) and also for a cyclic universe model. For the inflationary models the calculation of quasiparticle production $N_{k}$ shows the following trends:

(i) $N_{k}$ is enhanced for faster expansions (small $t_{s}$ ) and larger expansions (large $X$ ). In the limit of a very fast expansion, the results approach the sudden result from (6.95), which is expected since the field does not evolve for a sufficiently fast change in the nonlinearity. Quasiparticle production is always suppressed below the sudden prediction.

(ii) $N_{k}$ is larger for small momenta, since each mode is strongly coupled to the effectivespacetime in this case. Alternatively stated, the field equation (5.14) becomes adiabatic for large momenta, so that these modes are not strongly excited by the expansion. Hence the classical field simulations demonstrate the effects of Lorentz violation for the effective spacetime. As expected, the analytic predictions within the acoustic approximation agree favourably with the quasiparticle production for small momenta.

Finally, for the case of a cyclic universe, quasiparticle production is entirely attributed to parametric excitation of the modes as there is no net change to the effective spacetime. In particular, parametric resonance is observed for a mode $\omega(k)=\Omega / 2$ where $\Omega$ is the driving frequency. The parametric resonance peak broadens in momentum space for larger driving amplitude $\Delta$ as expected from the analysis in [207].

Our calculations (both analytical and numerical) clearly indicate that quasiparticle production should occur in a number of different scenarios, but it is worth commenting on relevant experimental studies that have appeared in the literature, and to then suggest possible extensions to our simple model.

\subsubsection{Possible experimental implementation}

While a specific experiment corresponding to the analogue FRW model we have described has not yet been implemented, there has been significant progress in experiments which might ultimately lead to this goal. In particular, the two main features required by our model are: (i) a homogeneous BEC in a box trap; and (ii) a time-varying scattering length.

In particular, some progress has been made in experimental implementations of a box trap for BECs, including a square well potential with high barriers on an atom chip [217] and a novel optical trap [218]. It should be noted, however, in both these experiments the resulting hard-wall potential confined the condensate in only one dimension. On the other hand, the implementation of a time-varying scattering length is easily achievable in a number of different atomic species by making use of a Feshbach resonance $[137,138]$. As noted in section 7.4 , a promising candidate in this regard is ${ }^{85} \mathrm{Rb}$ which allows widely tunable interactions [16, 212]. However, it should be emphasised that there are additional complications near a Feshbach resonance from either the inelastic processes of three-body recombination (TBR) [219], molecule formation [139] or the existence of Efimov states [220]. It should be possible to avoid these issues in experiments by 
avoiding tuning the interactions too closely to the Feshbach resonance, or by expanding on a time scale too fast for TBR to have an appreciable effect.

Irrespective of the exact details for the experimental realisation of the FRW analogue model — ie. for a homogeneous condensate in a box trap - we should apply either (or both) of the conditions: (i) that the potential at the edge of the box satisfies $V_{\mathrm{box}} \gg U n$, or (ii) that the time-scale of expansion should be very small compared with the trapping frequency so that the condensate remains in the ground state of the trap.

Finally, it is worth commenting that there is already experimental evidence for excitation of a condensate (ie. quasiparticle production) due to a time-varying scattering length. In one such experiment by Claussen et al. [221], Bose condensed ${ }^{85} \mathrm{Rb}$ atoms were subjected to an increase in scattering length, followed by a hold time and then a reduction in scattering length. In that work the resulting depletion of the condensate increased with decreasing rise time, except for small hold times $\left(t_{\text {hold }} \leq 15 \mu \mathrm{s}\right)$ and small rise times $\left(t_{\text {rise }} \lesssim 20 \mu \mathrm{s}\right)$. The time scale over which the scattering length was modified was too small for the condensate shape to adjust dynamically. This experiment would therefore correspond to a contraction and expansion of an effective spacetime, and the dependence of the condensate particle loss on the rise time is consistent with our predictions of quasiparticle production. This effect has been reproduced in numerical simulations by using a generalised Gross-Pitaevskii equation [222], although it was necessary to include the effects of TBR there because of the relatively long hold times.

\subsubsection{Outlook}

The FRW analogue model we have considered is based on several simplifying assumptions, the two most significant being homogeneity of the condensate and the two-dimensional box geometry. The preceding discussion therefore motivates several directions in which the formalism could be extended to deal with any realistic experiments that would implement a FRW analogue model of an expanding unverse:

1. In an experimental implementation of the FRW analogue model, the actual trapping potential may differ from a two-dimensional box trap — specifically, an implementation will likely require a three-dimensional system with a non-zero potential. The extension of the classical field simulations from two to three dimensions is straightforward since the PGPE (7.5) takes the same form in either case. Moreover, it is straightforward to include a realistic trap into the classical field simulations by specifying the non-zero potential in the PGPE. However, due to the significant increase in size of the mode space for three dimensions, these simulations would necessarily require a considerable computational effort (ie. running trajectories in parallel on a cluster of workstations).

2. As an alternative to the condensate in a box scenario, we might consider the case of a BEC in a harmonic trap where the trapping frequency and scattering length are simultaneously modified in such a way that the condensate density is approximately constant at the center of the trap. Thus the FRW analogue model we have considered could be approximately reproduced near the center of the trap. 
3. It may be necessary to include the effects of TBR to accurately describe the dynamics close to a Feshbach resonance. The inclusion of TBR into the TWA has been previously described by Norrie et al. [223].

4. Finally, the presence of a thermal cloud will certainly affect the results of any experiment, possibly obscuring the signal of quasiparticle production due to expansion. Finite temperature effects may be included by using the classical field method whereby the phase space is separated into a coherent region (highly occupied modes) and an incoherent region (weakly occupied modes). This has been formalised in terms of either the finite temperature GPE [53] or the stochastic GPE [55].

Whether or not any of the above modifications are incorporated into the model, however, one should remain wary that the analogy to a FRW-type universe is preserved in some regime of interest. 


\section{Part III}

An analogue model of an acoustic Black Hole in Bose-Einstein condensates 



\section{Chapter 9}

\section{The quantum de Laval nozzle}

The mathematical facts worthy of being studied are those which, by their analogy with other facts, are capable of leading us to the knowledge of a physical law.

Jules H. Poincare

\subsection{Introduction}

\subsubsection{Background}

One of the more interesting realisations of an analogue model of gravity is the formation of sonic horizons in a fluid. The general consensus is that, within certain approximations, such systems should exhibit Hawking radiation in analogy with cosmological black holes. In this part of the thesis, we will introduce and analyse a specific proposal for an acoustic black hole in a BoseEinstein condensate: the quantum de Laval nozzle. Our treatment utilises methods of many-body quantum field theory, including effects beyond the mean-field description. Indeed a fundamental issue for the prediction of Hawking radiation is the role of quantum noise inasmuch as the vacuum state allows processes that lead to particle production.

\subsubsection{Hawking Radiation in analogue models}

In chapter 5 we introduced the idea of analogue gravity, which includes the possibility of observing the analogue of the Hawking effect in fluid systems exhibiting sonic horizons, an idea first put forward by Unruh [103]. In that paper, using an analysis similar to Hawking's original analysis for cosmological black holes $[162,163]$, Unruh showed an acoustic black hole should emit sound waves with a Planckian spectrum at the Hawking temperature

$$
k_{B} T_{H}=\frac{\hbar g_{H}}{2 \pi c_{H}}
$$

where $g_{H}$ is the surface gravity at the black hole horizon and $c_{H}$ is the speed of sound at the horizon. (Note that this form of the Hawking temperature is from Visser [118] although it is identical to the result from Unruh [103].) The derivation required the quantisation of a scalar field propagating in a classical fluid, analogous to a classical gravitational field. The study of 
acoustic black holes in classical fluids has subsequently been developed by a number of authors $[118,130,173,188,224]$.

The translation of this prediction to Bose-Einstein condensates (BECs), is motivated not least by the observation that the long wavelength description of the system is given by hydrodynamical equations of motion - this is the acoustic approximation. There are other good reasons that BECs are promising candidates for analogue models, which we have previously discussed in section 5.4.4. To date, several groups have undertaken theoretical investigations of sonic horizons and the Hawking effect in BECs, most notably:

Garay and co-workers [149,161]: Garay et al. first considered the possibility of using BECs as a candidate system for studying sonic horizons [149,161]. In particular, they analysed two configurations that have sonic horizons: (i) a black and white hole horizon pair formed in a effectively one-dimensional ring geometry; and (ii) a sink-generated acoustic black hole in an infinite one-dimensional condensate. For the ring geometry, they used a periodic ansatz for the condensate density of the form

$$
\rho(\theta)=\frac{N}{2 \pi}(1+b \cos \theta)
$$

where $N$ is the total number of atoms in the condensate, $b$ is some parameter such that $b \in[0,1], \theta$ is the azimuthal angle around the ring, and where periodicity requires an integer winding number. Using this ansatz, they performed a linear stability analysis with the GPE and found regions in parameter space that exhibited dynamical instabilities, which are associated with quasiparticle creation. However, they suggest that [149] "Evaporation through an exponentially self-amplifying instability is not equivalent, however, to the usual kind of Hawking radiation".

Barcelo and co-workers [135, 142]: Barcelo et al. have proposed using a pair of de Laval nozzles to produce a region of supersonic flow bounded by two subsonic regions [135, 142]. In particular, the flow becomes supersonic after the first nozzle, whereas it becomes subsonic after the second nozzle. This configuration is equivalent to a black hole-white hole horizon pair. They found a single nozzle leads to an estimate for the Hawking temperature of

$$
k_{B} T_{H}=\hbar \frac{c_{H}}{2 \pi \sqrt{A_{H}}} \sqrt{\frac{3 A_{H}^{\prime \prime}}{4}}
$$

valid in the acoustic approximation, where $c$ is the speed of sound, $A$ is the cross-sectional area of the nozzle and the subscript $H$ for values on the right hand side mean the corresponding value should be evaluated at the horizon. An estimate of the size of the effect in Bose-Einstein condensates then gives a Hawking temperature of $T_{H} \approx 70 \mathrm{nK}$, which may in principle be observable.

Leonhardt and co-workers [61,225]: Subsequent to the work of Garay et al. [149, 161], Leonhardt et al. [61] formulated a general theory of elementary excitations for unstable BECs. In particular, they extended the usual Bogoliubov theory for inhomogeneous con- 
densates (see $[29,226])$ to include the case when dynamical instabilities were present, and further outlined how to construct normalisable modes for the unstable case by taking linear combinations of the unstable modes. They then considered a one-dimensional model for a sonic horizon based on the relation

$$
u=-c+\alpha z
$$

valid close to the horizon. Here $u$ is the flow velocity, $c$ is the speed of sound, $\alpha$ is the velocity gradient at the horizon (and therefore related to the surface gravity), and $z$ is a spatial coordinate with the horizon at $z=0$. Moreover, a positive/negative value for $\alpha$ corresponds to a sonic black/white hole horizon respectively. Using the WKB (ie. semiclassical) approximation and the acoustic (ie. hydrodynamic) approximation, they showed that a sonic white hole horizon has a discrete spectrum of instabilities.

In a related paper [225] the same authors argue that the Hawking effect can be regarded as quantum depletion, seemingly in the absence of dynamical instabilities, and specifically that the interaction of elementary excitations via Landau-Baliaev damping plays the role of black hole evaporation.

Giovanazzi and co-workers [146]: Giovanazzi et al. [146] have considered the formation of a sonic horizon from an external potential applied to a BEC confined in a waveguide . Using the hydrodynamic approximation, they find the conditions for which a quasistationary sonic horizon forms, and therefore predict a Hawking temperature that depends on the horizon details and the mass of the constituent atoms.

\subsubsection{Outline}

The above investigations notwithstanding, it is still not entirely clear whether the prediction of the Hawking effect is valid for acoustic black hole geometries in quantum systems such as BECs. Inspired by this issue, in this final part of the thesis, we will introduce and analyse a specific geometry for an acoustic black hole in a BEC, formed by two de Laval nozzles in a ring configuration - a system we refer to as the quantum de Laval nozzle. While there certainly exist previous studies of de Laval nozzle geometries in the context of acoustic black holes, specifically for classical fluids $[143,145]$ and for BECs $[135,142]$, the analyses in these studies are either classical or semiclassical in nature. Our analysis utilises the effective quantum field theory for weakly interacting Bose gases. We make no assumptions a priori with regards to an analogue model for the system, except for the requirement of a sonic horizon. The results are therefore valid independently of their interpretation in terms of an analogue model for an acoustic black hole, although we will comment on the connection of this study with the prediction of the Hawking effect for analogue models, and in particular for BEC systems.

The outline of this part of the thesis is as follows. In this chapter, we introduce the quantum de Laval nozzle, a geometry that exhibits sonic horizons, and connect this with the notion of the analogue of the Hawking effect. We then find the transonic stationary states for our system by specifying a non-zero phase quantisation. In the next chapter, we solve the Bogoliubov-de Gennes 
equations to find the elementary excitations of our system. We are therefore able to characterise the stability of the system for a large range of parameters. The presence of dynamical instabilities provides - we believe - the closest connection to the Hawking effect as a dynamical process. The effect is then demonstrated by evolving the system using a phase space method based on the truncated Wigner approximation. We then discuss our results, conclude and suggest avenues for further enquiry.

\subsection{The de Laval nozzle}

The de Laval nozzle is introduced, and it is shown how this configuration can lead to sonic horizons due to transonic flow. We then propose a periodic arrangement of two such nozzles, which should lead to a stable configuration for a closed system.

\subsubsection{Nozzle equation}

We outline a generalised derivation of the well-known nozzle equation for the Laval Nozzle; see $[227,228]$ for example. We start with the equations of motion for a compressible fluid of local density $\rho$, velocity $\mathbf{v}$, pressure $p$ and in a external field of force $\mathbf{F}$ (the usual case is $\mathbf{F}=\mathbf{g}$ for a uniform gravitational field).

Continuity is given by:

$$
\frac{\partial \rho}{\partial t}+\nabla \cdot(\rho \mathbf{v})=0
$$

Euler's equation is given by:

$$
\frac{\partial \mathbf{v}}{\partial t}+\mathbf{v} \cdot \nabla \mathbf{v}=-\frac{\nabla p}{\rho}+\mathbf{F}
$$

Consider a de Laval nozzle that narrows to a waist ${ }^{1}$ and then widens - the cross-sectional area is denoted $A$. Moreover, we impose an external potential $V(\mathbf{x})$ so that the external force is $\mathbf{F}=-\nabla V(\mathbf{x}) / m$.

For simplicity, we further consider an effective one dimensional geometry where $A$ and $V$ only depend on the $x$ coordinate. For a steady state (dropping the time derivatives), continuity (9.5) becomes

$$
\rho A v=\text { constant }
$$

The differential form is

$$
\frac{d v}{v}+\frac{d A}{A}+\frac{d \rho}{\rho}=0
$$

\footnotetext{
${ }^{1}$ the term throat is also used in the literature to denote the narrowest point of the nozzle
} 
Noting that $c^{2} \equiv d p / d \rho$ for isentropic flow, Euler's equation (9.6) in differential form is

$$
v d v+c^{2} \frac{d \rho}{\rho}+\frac{d V(x)}{m}=0
$$

Combining equation (9.8) with (9.9) then yields the nozzle equation:

$$
\frac{d v}{v}=\left(\frac{c^{2}}{c^{2}-v^{2}}\right)\left(\frac{d V(x)}{m c^{2}}-\frac{d A}{A}\right)
$$

We are now in a position to consider two specific cases.

\section{Constant external potential $V$}

The usual de Laval nozzle corresponds to the case where the external potential can be neglected. In this case (9.10) becomes

$$
\frac{d v}{v}=-\left(\frac{c^{2}}{c^{2}-v^{2}}\right) \frac{d A}{A}
$$

The physical consequences of the nozzle equation are thus as follows:

1. The situation where $v=c$ is clearly only permitted where $d A=0$, that is at the waist of the nozzle.

2. For subsonic flow $(v<c)$ :

When $d A<0$ the velocity is increasing.

When $d A>0$ the velocity is decreasing.

3. For supersonic flow $(v>c)$ :

When $d A<0$ the velocity is decreasing.

When $d A>0$ the velocity is increasing.

The consequence of this behaviour is that if the flow has a finite velocity (and is therefore stable), it must adjust so that $v=c$ at the waist of a nozzle. Therefore if a subsonic flow approaches the waist, becoming supersonic at the waist, the flow is supersonic after exiting the waist. Conversely, if a supersonic flow approaches the waist, becoming subsonic at the waist, the flow is subsonic after exiting the waist.

\section{Constant cross-sectional area $A$}

Alternatively, we consider a constant $A$ and include an external potential $V$. In this case (9.10) becomes

$$
\frac{d v}{v}=\left(\frac{c^{2}}{c^{2}-v^{2}}\right) \frac{d V}{m c^{2}}
$$

Similarly to above, the physical consequences of this form of the nozzle equation are as follows: 
1. The situation where $v=c$ is clearly only permitted where $d V=0$, that is at the waist of the nozzle.

2. For subsonic flow $(v<c)$ :

When $d V<0$ the velocity is decreasing.

When $d V>0$ the velocity is increasing.

3. For supersonic flow $(v>c)$ :

When $d V<0$ the velocity is increasing.

When $d V>0$ the velocity is decreasing.

Similarly to the discussion above, we can assign the point where $d V=0$ as the waist of the nozzle, the flow adjusting so that $v=c$ at the waist. If a subsonic flow approaches the waist of a nozzle, becoming supersonic at the waist, the flow is supersonic after exiting the waist. Conversely, if a supersonic flow approaches the waist, becoming subsonic at the waist, the flow is subsonic after exiting the waist.

\subsubsection{The quantum de Laval nozzle}

In a toroidal geometry to achieve steady flow which is transonic, two de Laval nozzles are required in tandem, the flow becoming supersonic at the waist of the first, and then subsonic at the waist of the second. This configuration corresponds to the formation of both a black and white hole horizon.

To implement such a nozzle we consider the one dimensional problem where the current $\mathbf{j}=$ $n \mathbf{v}$ is constant and where there is external potential of the form

$$
V(x)=V_{0} \cos ^{2}\left(\frac{2 \pi x}{L}\right)
$$

which has periodicity 2 over the region $-L / 2 \leq x \leq L / 2$.

For a BEC confined by such a potential, the stationary states (and so the effective spacetime geometry) are found by solving the time-independent Gross-Pitaevskii equation subject to phase quantisation. This may lead to modifications of the usual hydrodynamic solutions, and we therefore denote this configuration as the quantum de Laval nozzle (QdLN).

To extend the above analysis to three (or two) spatial dimensions it is necessary to include variations in both $A$ and $V$. Formally the one dimensional configuration is reached by starting from the equations of motion and integrating over the transverse dimensions. The validity of the resulting one dimensional equations require the assumption that the spatial extent of the condensate in the transverse dimensions is small compared with the circumference of the torus, but is large enough to ensure phase coherence over the condensate (ie. larger than the healing length) and for the scattering to be three-dimensional (ie. larger than the scattering length $a$ ). 


\subsection{Estimated Hawking temperature}

Treating the effective spacetime geometry as a classical quantity, so that a continuum of modes are available, we are able to apply the usual reasoning to estimate the Hawking temperature for our system. Specifically, following Visser $[118,170]$ we define the surface gravity for an sonic hole as

$$
g=-\frac{1}{2} \frac{d\left(c^{2}-v^{2}\right)}{d x}
$$

An analytic prediction for the Hawking temperature (9.1) for a nozzle with a spatially varying cross-sectional area has been found previously [135, 142]. A similar analysis for a nozzle formed by an external potential can be made by considering the nozzle equation (9.12). In this case the surface gravity at the horizon is given by

$$
g_{H}= \pm c_{H} \sqrt{\frac{-\left.3 \nabla^{2} V\right|_{H}}{4 m}}
$$

where L'Hospital's rule has been used and where $\left.\nabla^{2} V\right|_{H}$ denotes the Laplacian of V is evaluated at the horizon. The estimated Hawking temperature from (9.1) is then

$$
k_{B} T_{H}=\frac{\hbar}{4 \pi} \sqrt{\frac{-\left.3 \nabla^{2} V\right|_{H}}{m}}
$$

Using the periodic potential (9.13) and considering the phonon part of the spectrum with $\hbar \omega_{k} \approx k c$ we find

$$
\omega_{\text {peak }}=\sqrt{\frac{3 V_{0}}{8 m L^{2}}}
$$

so that the Hawking radiation spectrum peaks at the wavelength

$$
\lambda_{\text {peak }}=2 \pi c_{H} \sqrt{\frac{8 m L^{2}}{3 V_{0}}}
$$

This result is based on the equations of motion for a classical fluid, where the quantum pressure term has been neglected - this is the hydrodynamic approximation. The prediction of a thermal spectrum at the temperature given by (9.16) only applies to the phononic part of the spectrum.

\subsection{Stationary states}

In this section we find stationary solutions for the QdLN, which exhibits a black and white hole pair; this formulation includes the quantum pressure term and enforces the periodicity of the system. 


\subsubsection{Equations of motion}

The model is formulated using the one dimensional Gross-Pitaevskii equation (GPE)

$$
i \hbar \frac{\partial \psi(x, t)}{\partial t}=\left(-\frac{\hbar^{2} \partial_{x}^{2}}{2 m}+V(x)+U_{1 \mathrm{D}}|\psi(x, t)|^{2}\right) \psi(x, t)
$$

which is obtained from (1.8) by assuming the the transverse wavefunction is in the harmonic oscillator ground state given by $\phi(r)=\left(1 / \pi r_{\perp}^{2}\right)^{1 / 2} e^{-r^{2} / 2 r_{\perp}^{2}}$. The effective nonlinearity is $U_{1 \mathrm{D}}=$ $U /\left(4 \pi r_{\perp}^{2}\right)$ which is valid assuming the scattering length $a$ is much smaller than the transverse dimension $r_{\perp}$ so that the scattering is effectively three dimensional. Assuming the wavefunction can be written as a macroscopic order parameter

$$
\psi(x, t)=\sqrt{n(x, t)} e^{i \Theta(x, t)}
$$

with current density

$$
j \equiv \frac{\hbar}{2 m i}\left(\psi^{*} \nabla \psi-\psi \nabla \psi^{*}\right)
$$

This definition gives the velocity

$$
v=\frac{\hbar}{m} \partial_{x} \Theta
$$

Substituting into (9.19) the equations of motion are

$$
\frac{\partial n}{\partial t}+\partial_{x}(n v)=0
$$

and

$$
-\hbar \frac{\partial \Theta}{\partial t}=-\frac{\hbar^{2}}{2 m \sqrt{n}} \partial_{x}^{2} \sqrt{n}+V(x)+U_{1 \mathrm{D}} n+\frac{1}{2} m v^{2}
$$

When the interaction term dominates, the density varies slowly, and the Laplacian term $\partial_{x}^{2}$ (ie. the quantum pressure term) can be dropped; then using (9.22) this last equation can be rewritten as

$$
m \frac{\partial v}{\partial t}=-\partial_{x}\left(V(x)+U_{1 \mathrm{D}} n+\frac{1}{2} m v^{2}\right)
$$

\subsubsection{Ground state}

For steady state flow we take the one dimensional stationary solution of the form

$$
\psi(x, t)=\sqrt{n(x)} e^{i \vartheta(x)} e^{-i \mu t / \hbar}
$$

If we take the stationary solution of (9.23) and (9.24) using (9.26), and take the fixed current condition, we can write:

$$
\begin{aligned}
n & \equiv s^{2} \\
v & =j / s^{2} \\
\mu s & =-\frac{\hbar^{2}}{2 m} \frac{d^{2} s}{d x^{2}}+V(x) s+U_{1 \mathrm{D}} s^{3}+\frac{m j^{2}}{2 s^{3}}
\end{aligned}
$$


Solutions to this nonlinear Schrödinger equation then allow us to specify the wavefunction by:

$$
\begin{aligned}
\vartheta(x) & =\frac{m}{\hbar} \int \frac{j d x}{s(x)^{2}} \\
\psi(x) & =s(x) \exp (i \vartheta(x))
\end{aligned}
$$

To ensure the complex wavefunction is single-valued on the periodic domain, we must also apply the phase quantisation condition:

$$
\Delta \vartheta=\frac{m}{\hbar} \int_{-L / 2}^{L / 2} v(x) d x=2 \pi w_{0}
$$

for an integer winding number given by $w_{0}$ (a topological quantum number).

\subsubsection{Solutions in hydrodynamic approximation}

Assuming the interactions dominate (ie. the nonlinear term is large), the density varies slowly and the Laplacian term can be dropped - this is the hydrodynamic approximation. In this case the time-independent GPE can be written as a cubic, either in terms of the density:

$$
n^{3}+\left(\frac{V(x)-\mu}{U_{1 \mathrm{D}}}\right) n^{2}+\frac{m j^{2}}{2 U_{1 \mathrm{D}}}=0
$$

or (using $v=j / n$ ) in terms of the velocity :

$$
v^{3}+2\left(\frac{V(x)-\mu}{m}\right) v+\frac{2 j U_{1 \mathrm{D}}}{m}=0
$$

No flow $(j=0)$ :

In this case $v=0$ and there is one non-trivial solution

$$
n=\frac{\mu-V(x)}{U_{1 \mathrm{D}}}
$$

We normalise to the single-particle wavefunction so that $\int n d x=1$, the chemical potential $\mu$ being adjusted to enforce this condition. The normalisation condition is given by

$$
\int_{-L / 2}^{L / 2} \frac{\mu-V_{0} \cos ^{2}(2 \pi x / L)}{U_{1 \mathrm{D}}} d x=1
$$

which gives

$$
\mu=\frac{U_{1 \mathrm{D}}}{L}-\frac{V_{0}}{2}
$$

The density then takes the analytic form

$$
n=\frac{1}{L}+\frac{V_{0}}{U_{1 \mathrm{D}}}\left(\frac{1}{2}-\cos ^{2}(2 \pi x / L)\right)
$$




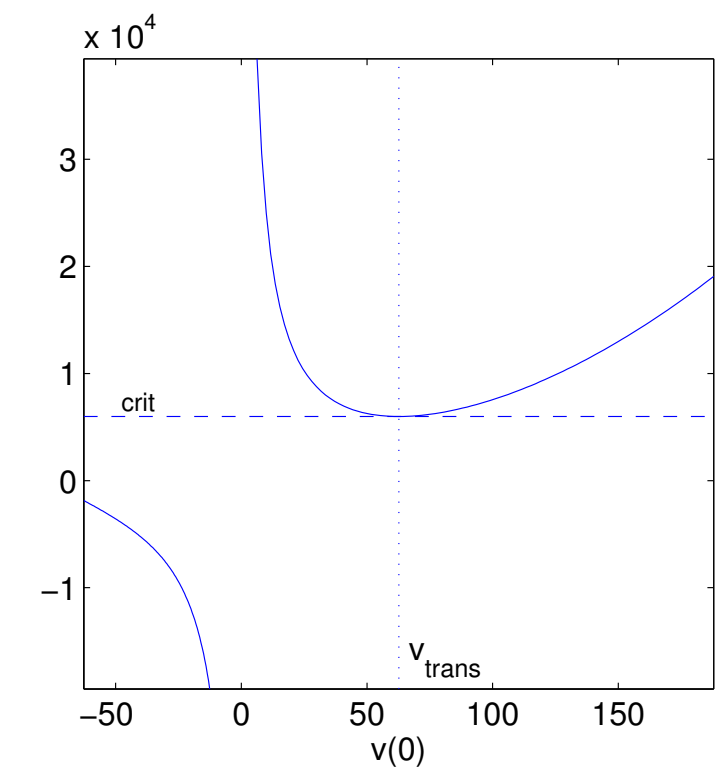

Figure 9.1: The chemical potential as a function of flow velocity at the waist $(x=0)$ as given by (9.34); when $\mu>\mu_{\text {crit }}$ there are two real positive solutions. The dashed line indicates the transonic condition where $c=v$ and a crossover is possible from the subsonic to supersonic regimes (or vice versa). Dimensionless parameters are $C=500, V_{0}=100$ and $w_{0}=10$ (the current is determined implicitly by this choice of parameters).

Non-zero flow $(j>0)$ :

For the case where there is non-zero flow $(j>0)$, we find solutions using (9.34) since the equations have a simpler form in this case.

We must select our parameters appropriate to ensure we can find a configuration with a crossover between subsonic and supersonic regimes. The solutions to a cubic of the form $v^{3}+a_{2} v^{2}+a_{1} v+a_{0}=0$ are characterised by defining the quantities [202]

$$
q=\frac{1}{3} a_{1}-\frac{1}{9} a_{2}^{2}, \quad r=\frac{1}{6}\left(a_{1} a_{2}-3 a_{0}\right)-\frac{1}{27} a_{2}^{3}
$$

and then noting that

$$
\begin{aligned}
& q^{3}+r^{2}>0 \rightarrow 1 \text { real and } 2 \text { complex roots } \\
& q^{3}+r^{2}=0 \rightarrow \text { all roots real, at least } 2 \text { equal } \\
& q^{3}+r^{2}<0 \rightarrow \text { all roots real }
\end{aligned}
$$

From the cubic (9.34) we can write

$$
q^{3}+r^{2}=\frac{8}{27}\left(\frac{V(x)-\mu}{m}\right)^{3}+\left(\frac{j U_{1 \mathrm{D}}}{m}\right)^{2}
$$

Clearly a crossover from the subsonic to supersonic branches at a particular point, $x_{\text {trans }}$ say, will only occur when $q^{3}+r^{2}=0$. Moreover stable flow will only occur throughout the region 
when the subsonic and supersonic solutions are real everywhere (ie. $q^{3}+r^{2} \leq 0$ ). From (9.39), it is only possible to satisfy both these constraints when the transonic point occurs at the maximum value of the potential $V\left(x_{\text {trans }}\right)=V_{0}$. That is, the maximum of the potential acts as the waist of the Laval nozzle.

Choosing $q^{3}+r^{2} \leq 0$, so that there are always at least two real solutions, we can express the solutions analytically as:

$$
\begin{array}{ll}
v_{n}(x)=\sqrt{\frac{8(\mu-V(x))}{3 m}} \cos \left(\frac{\theta(x)+2 \pi}{3}\right) & \text { negative } \\
v_{-}(x)=\sqrt{\frac{8(\mu-V(x))}{3 m}} \cos \left(\frac{\theta(x)+4 \pi}{3}\right) & \text { subsonic } \\
v_{+}(x)=\sqrt{\frac{8(\mu-V(x))}{3 m}} \cos \left(\frac{\theta(x)}{3}\right) & \text { supersonic }
\end{array}
$$

with

$$
\theta(x)=\cos ^{-1}\left(-\frac{j U_{1 \mathrm{D}}}{m}\left[\frac{3 m}{2(\mu-V(x))}\right]^{3 / 2}\right)
$$

We can re-express the condition for transonic flow as either of the following choices of parameters:

1. If we specify $U_{1 \mathrm{D}}, V(x)$ and $\mu$ the critical current is

$$
j_{\text {crit }}=\sqrt{\frac{8}{27 U_{1 \mathrm{D}}^{2} m}}\left(\mu-V_{0}\right)^{3 / 2}
$$

For $j>j_{\text {crit }}$ we have $q^{3}+r^{2}>0$ and the flow is unstable.

2. Alternatively if we specify $U_{1 \mathrm{D}}, V(x)$ and $j$ (and assuming these quantities are real and positive) the critical chemical potential is

$$
\mu_{\text {crit }}=\frac{3}{2}\left(j U_{1 \mathrm{D}}\right)^{2 / 3} m^{1 / 3}+V_{0}
$$

For $\mu<\mu_{\text {crit }}$ we have $q^{3}+r^{2}>0$ and the flow is unstable.

\section{Finding transonic solutions}

We can find a transonic solution by taking the chemical potential $\mu=\mu_{\text {crit }}\left(x_{\text {trans }}\right)$ so that there is a crossover from the subsonic to supersonic branches; the transonic solution is then constructed by conjoining the subsonic $\left(v_{-}\right)$and supersonic $\left(v_{+}\right)$solution branches. Without loss of generality, we take the subsonic branch to span the interval $x \in[-L / 2,0]$. More specifically, we have the following prescription:

1. For a given $V(x), U_{1 \mathrm{D}}$ and an initial value of $j$ calculate the critical chemical potential (9.45) for crossover at $V\left(x_{\text {trans }}\right)=V_{0}$ 


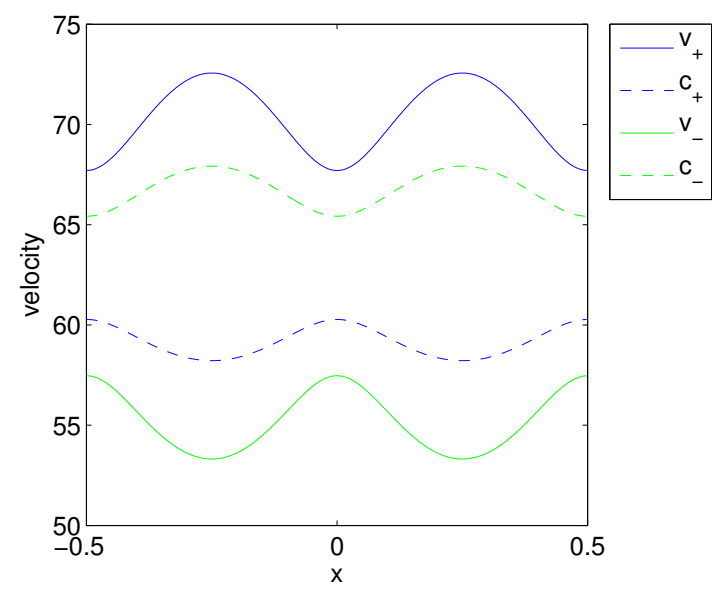

(a) $J=0.99 J\left(w_{0}=10\right)$

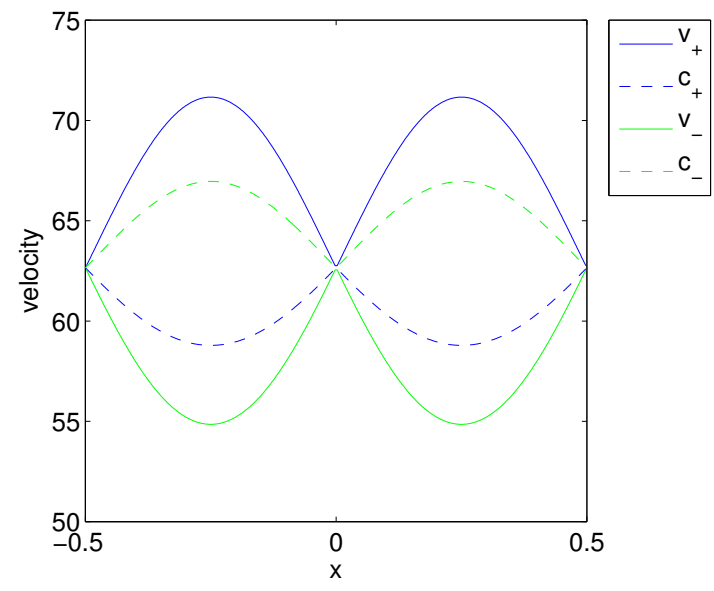

(b) $J=J\left(w_{0}=10\right)$

Figure 9.2: velocity $(v)$ and speed of sound $(c)$ for two real and positive solutions to cubic given by (9.41) and (9.42); in both cases parameters are $C=500, V_{0}=100$ and $\mu=\mu_{\text {crit }}=5.99 \times 10^{3}$ for the case when $J=J\left(w_{0}=10\right)$. The third solution of the cubic is negative, giving a negative density, and is therefore omitted. When $J>J\left(w_{0}=10\right)$ the solutions become complex indicating an unstable flow. Note that for the critical case (b) the current corresponds to a winding number of $w_{0}=10$, whereas for the subcritical case (a) phase quantisation is violated.

2. Use (9.40)-(9.42) and (9.43) to specify the subsonic and supersonic solution branches.

3. Combine the subsonic branch in the interval $-L / 2 \leq x \leq 0$ and the supersonic branch in the interval $0 \leq x \leq L / 2$.

4. Calculate the density and normalisation for this hybrid solution using $j=n(x) v(x)$

5. Iteratively adjust $j$ so that the hybrid solution satisfies the phase constraint (9.32) for a given winding number $w_{0}$.

Note that the normalisation of the resulting solution is determined by the choice of parameters $V_{0}, U_{1 \mathrm{D}}$ and $w_{0}$. It is worth commenting that the existence of the transonic solution entails a spontaneous breaking of the parity symmetry; that is, although $V(x)=V(-x)$, the transonic solution has $n(x) \neq n(-x)$.

We now use the transonic solution to the hydrodynamic problem as a starting point to finding the stationary solutions of the GPE including the so-called quantum pressure term.

\subsubsection{Dimensionless units}

Before proceeding further, it is useful to introduce dimensionless units. Defining the computational units for length, time and $s$, respectively as

$$
\begin{aligned}
x_{0} & =L \\
t_{0} & =m L^{2} / \hbar \\
s_{0} & =\sqrt{N_{0} / x_{0}}
\end{aligned}
$$


we introduce the dimensionless units

$$
\begin{aligned}
\bar{s} & =s / s_{0} \\
\bar{x} & =x / x_{0} \\
\bar{t} & =t / t_{0}
\end{aligned}
$$

Note that $\bar{s}$ is the dimensionless wavefunction normalised to unity. The equation of motion (9.29) can then be written as

$$
\bar{\mu} \bar{s}=-\frac{1}{2} \frac{d^{2} \bar{s}}{d \bar{x}^{2}}+\bar{V}_{0} \cos ^{2}(2 \pi \bar{x}) \bar{s}+C \bar{s}^{3}+\frac{J}{\bar{s}^{3}}
$$

with

$$
\begin{aligned}
\kappa & =m L^{2} / \hbar^{2} \\
\bar{\mu} & =\kappa \mu \\
\bar{V}_{0} & =\kappa V_{0} \\
C & =\frac{\kappa U_{1 \mathrm{D}} N_{0}}{L} \\
J^{2} & =\left(\frac{\kappa m L^{2}}{N_{0}^{2}}\right) j^{2}
\end{aligned}
$$

and where $\kappa$ is the unit of energy. Note that these units and the choice of potential ensure periodicity over the range $-1 / 2 \leq \bar{x} \leq 1 / 2$.

To facilitate numerical computation, we state the dimensionless form of several useful quantities. With the above choice of units the dimensionless velocity is $\bar{v}=(m L / \hbar) v$ which using $v=j / s^{2}$ gives

$$
\bar{v}=\frac{\sqrt{2 J}}{\bar{s}^{2}}
$$

Likewise the dimensionless speed of sound is $\bar{c}=(m L / \hbar) c$ which using $c=\sqrt{U_{1 \mathrm{D}} n / m}$ gives

$$
\bar{c}=\sqrt{C \bar{s}^{2}}
$$

The phase is given by

$$
\vartheta(\bar{x})=\int \bar{v} d \bar{x}
$$

Finally, the dimensionless form of the Hawking temperature (9.16) is given by

$$
\bar{T}=\frac{\bar{g}_{H}}{2 \pi \bar{c}_{H}}
$$

We can calculate this quantity from our stationary solutions by numerically evaluating (9.14) at the horizon.

In what follows we drop the bar notation for the dimensionless quantities; the subsequent use of either natural or dimensionless units will be clear from the context in which they appear. 


\subsubsection{Solving for the eigenstates}

To calculate stationary solutions of the time-independent Gross-Pitaevskii equation (9.19) we utilise a numerical method based on constrained optimisation. In particular, we formulate the problem as the the minimisation of the Gross-Pitaevski functional for a fixed nonlinearity $C$, potential depth $V_{0}$ and current $J$, subject to a phase quantisation constraint in terms of a fixed winding number $w_{0}$. Related methods of finding ground state solutions for the time-independent GPE are well documented by Blakie [229].

\section{Optimisation method}

Here the time-independent GPE is solved using a variational procedure with any additional constraints such as normalisation or phase circulation; in particular here we formulate problem as stated above in terms of $s(x)$ and specified current $J$ (which corresponds to a given phase quantisation). In particular, using (9.52), (9.58) and (9.60) and we recast the problem as a set of algebraic equations:

$$
\begin{aligned}
{\left[\frac{1}{2} \frac{d^{2}}{d x^{2}}+\mu-V(x)-C s(x)^{2}-\frac{J}{s(x)^{4}}\right] s(x) } & =0 \\
\int_{-L}^{+L} \frac{J}{s(x)^{2}} d x-2 \pi w & =0
\end{aligned}
$$

Note, the normalisation condition $\int n d x$ has been relaxed but will be re-instated after the optimisation by rescaling the nonlinearity $C$. The phase circulation constraint ensures a singlevalued wavefunction $\psi=s(x) e^{i \vartheta(x)}$ everywhere, including the boundary. By constructing the vector

$$
\mathbf{X}=\left\{s_{i}, \mu\right\}
$$

on the discrete spatial grid (with index $i$ for each point) we can write (9.62) as the matrix equation

$$
\mathbf{f}(\mathbf{X})=\mathbf{0}
$$

This equation is solved using the fsolve function from MATLAB, which is based on the MarquardtLevenberg algorithm. The initial condition $\mathbf{X}_{0}$ for the algorithm is supplied by the solution to the hydrodynamic problem as discussed in section 9.4.3. The fixed parameters of the problem are then given by $C, V_{0}$ and $w_{0}$. In contrast, the quantised current $J$ is determined by the solution to the hydrodynamic problem, and the chemical potential $\mu$ is determined by the optimisation procedure. As an additional step, the final solution is renormalised to a single-particle wavefunction by setting $\psi \rightarrow \psi / \sqrt{n_{0}}$ and $C \rightarrow C n_{0}$ where $n_{0}=\int s(x)^{2} d x$ is the density for the solution to the optimisation procedure. 


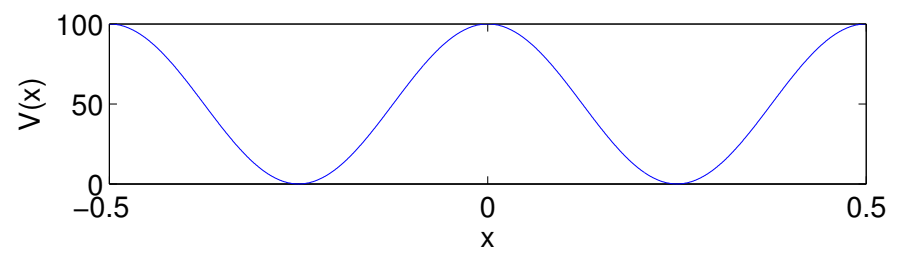

Figure 9.3: The potential (9.13) with $V_{0}=100$ (in dimensionless units, which have been introduced in section 9.4.4).

\section{Imaginary Time Propagation}

For completeness, although we do not utilise this method here, it is also worthwhile mentioning the method of imaginary time propagation (ITP) for finding stationary solutions. Here, a Wick rotation $\tau \rightarrow-i t$ transforms the GPE (9.19) into a diffusion equation so that during evolution the lowest eigenstate is damped at the slowest rate; hence this procedure is also known as the method of steepest descent. To preserve norm, the wavefunction requires renormalisation at every time step. The implementation of ITP for stationary states with persistent currents, which do not correspond to a global minimum, is problematic. In particular, it is difficult to enforce a topological condition on the solution such as a fixed winding number as the damped evolution drives the solution towards a true ground state (with zero current). Moreover, for the QdLN the problem cannot be recast in the rotating frame as translational invariance symmetry is broken by the periodic potential. We note however, that ITP has been used elsewhere to establish a non-zero current using a time-dependent potential [230].
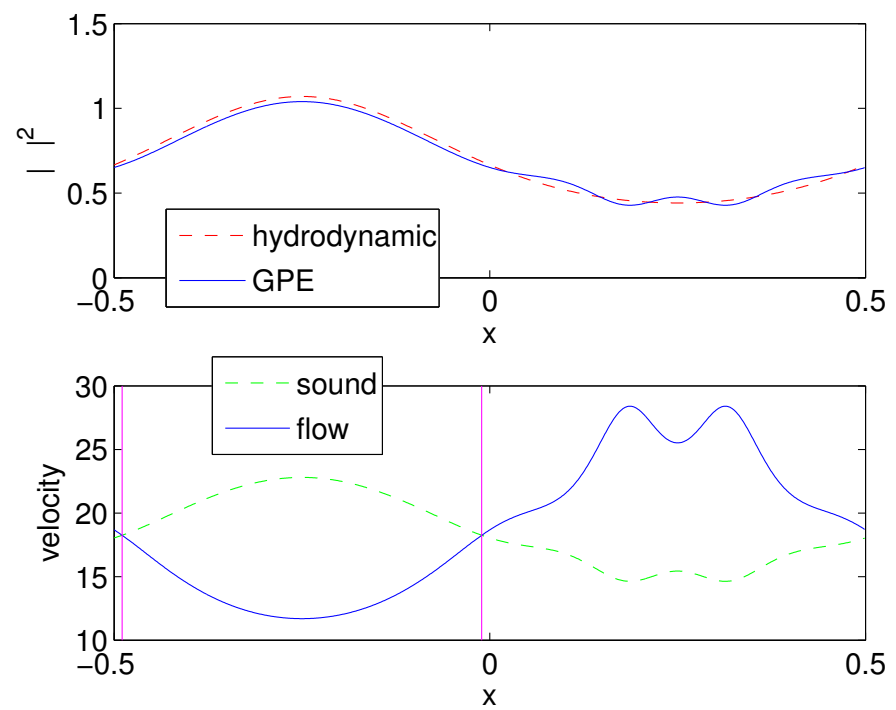

Figure 9.4: Stationary state for $w_{0}=3$ and $V_{0}=100$; corresponds to $\mu=5.93 \times 10^{2}$ and $C=3.51 \times 10^{2}$. 

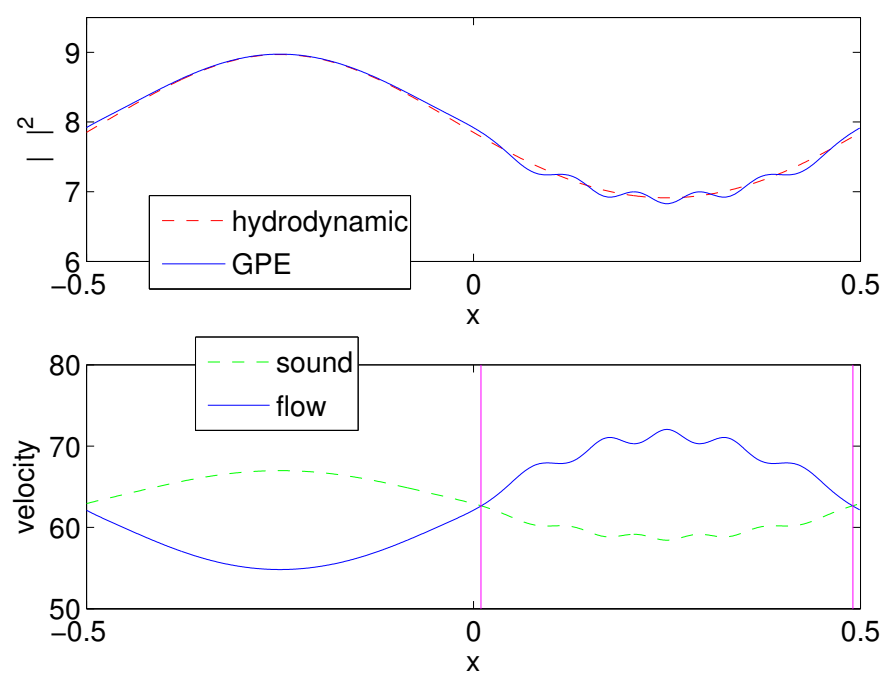

Figure 9.5: Stationary state for $w_{0}=10$ and $V_{0}=100$; corresponds to $\mu=5.99 \times 10^{3}$ and $C=3.95 \times 10^{3}$.
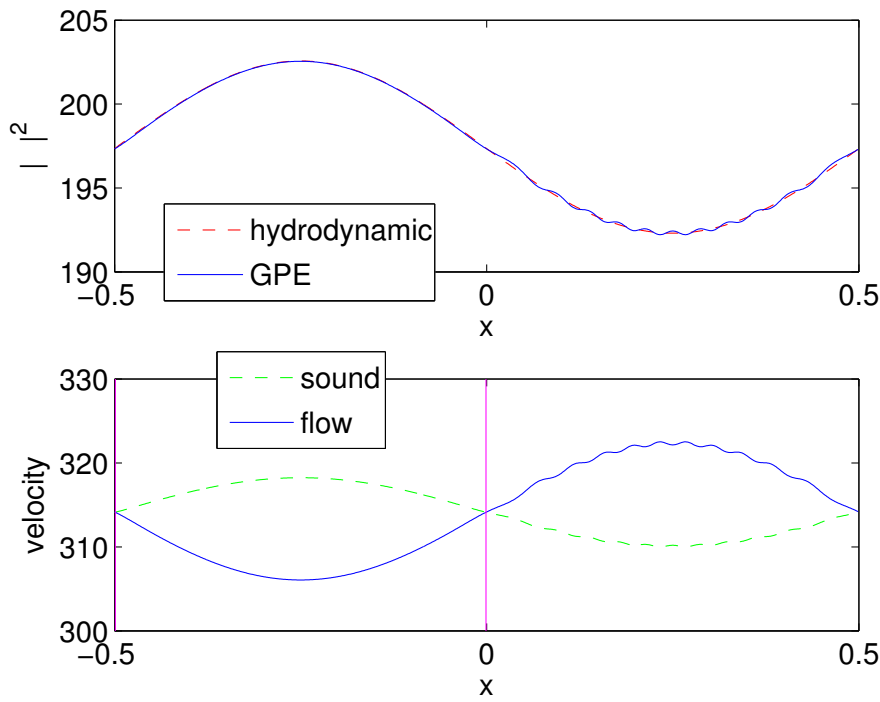

Figure 9.6: Stationary state for $w_{0}=50$ and $V_{0}=100$; corresponds to $\mu=1.48 \times 10^{5}$ and $C=9.87 \times 10^{4}$.

\subsection{Stationary solution results}

We can vary the parameters - winding number $w_{0}$ and potential depth $V_{0}-$ to find a range of transonic solutions for the QdLN. The winding number constraint specifies the current $J$ whereas normalisation of the solution to the single-particle wave function determines the nonlinearity $C$. We show solutions, using a potential with $V_{0}=100$ as shown in Figure 9.3, for three different winding numbers: (i) $w_{0}=3$ in Figure 9.4; (ii) $w_{0}=10$ in Figure 9.5; and (iii) $w_{0}=50$ in Figure 9.6. In each case, both the hydrodynamic and GPE stationary solutions are shown, as well as the resulting flow velocity and speed of sound for GPE stationary solution. The ergoregion 
$(v>c)$ is given approximately by the right hand region $0 \leq x \leq 0.5$. The result of using the GPE (which includes the quantum pressure term) to find the stationary states is a small adjustment in the positions of the black and white hole horizons when compared to the hydrodynamic case. By construction, in the hydrodynamic case, the black hole horizon occurs at $x_{\text {trans }}=0$, whereas the white hole horizon occurs at $x_{\text {trans }}= \pm 0.5$. For the case of the largest winding number $w_{0}=50$, shown in Figure 9.6, it is evident that this adjustment for the GPE stationary solutions is approximately zero because the hydrodynamic and GPE stationary solutions are approximately equal. We further note the presence of "ripples" in the ergoregion for the GPE stationary solutions, which are due to the quantum dispersion term, and which are larger in relative magnitude for low winding numbers ${ }^{2}$.

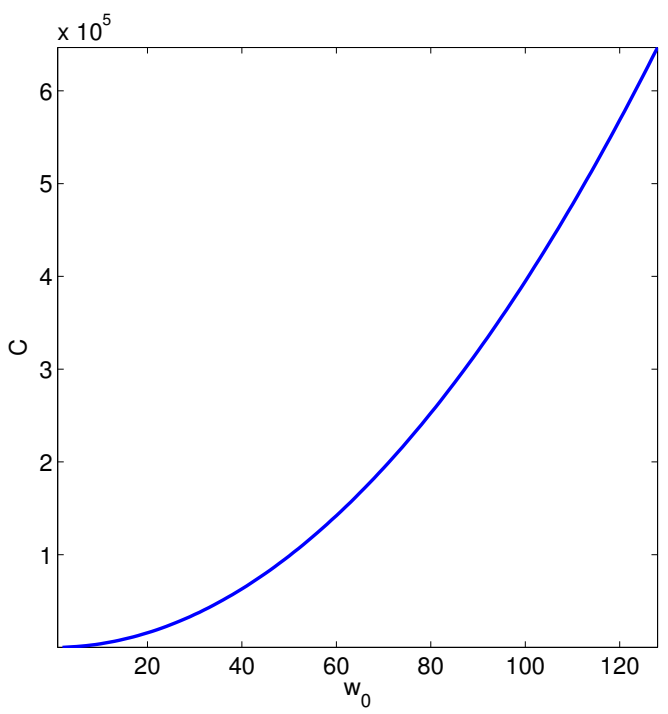

Figure 9.7: Nonlinearity $C$ as function of winding number $w_{0}$ for stationary solutions of QdLN with $V_{0}=100$. Note that the nonlinearity depends only very weakly on $V_{0}$, and reproducing this plot for any $V_{0} \leq 2000$ gives a quantitatively similar result.

The GPE stationary solutions are renormalised so that the nonlinearity $C$ is not a free parameter of the system (see section 9.4.5). In fact, numerical solutions indicate that $C$ increases with $w_{0}$ but depends only very weakly on $V_{0}$. We show the nonlinearity as a function of winding number in Figure 9.7 with $V_{0}=100$.

We have also calculated the estimated Hawking temperature for a range of parameters, as shown in Figure 9.8. Here the data points have been calculated from (9.61) by a numerical estimate of the surface gravity $\bar{g}_{H}$ for the GPE stationary solution, whereas the solid lines are given directly from the estimate (9.16) for the hydrodynamic theory. It is interesting to note that while the Hawking temperature is relatively insensitive to $w_{0}$, it increases with $V_{0}$. We emphasise that this estimate is based on the semi-classical picture of the system, and does not account for the quantised spectrum exhibited by the QdLN. We will comment further on the implications of this in the next

\footnotetext{
${ }^{2}$ Further investigation reveals the number of oscillations $n_{\mathrm{osc}}$ increases with either $w_{0}$ or $V_{0}$. One should be mindful that the GPE stationary solution is well represented by the numerical grid. In practice, using the Nyquist criterion, we have the requirement that the spatial grid should have at least $n_{x} \geq 4 n_{\text {osc }}$ points.
} 


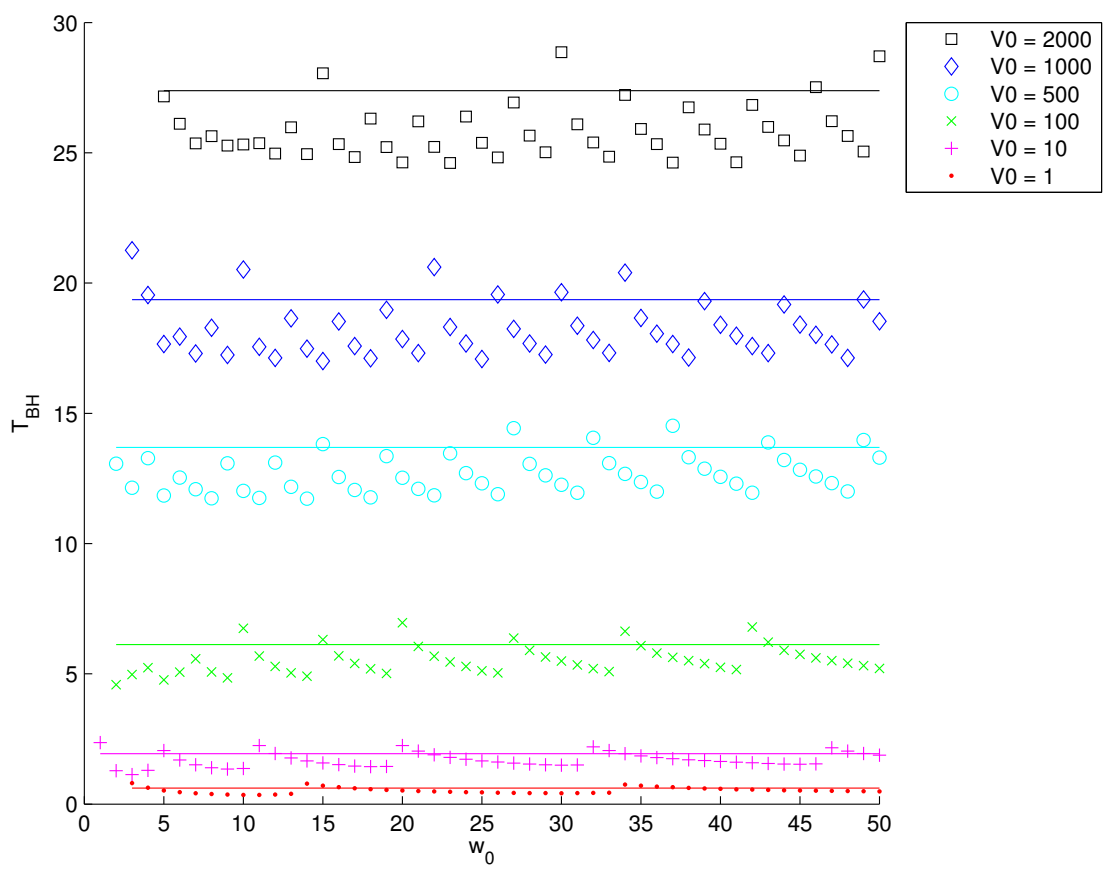

Figure 9.8: Estimated Hawking temperature as function of winding number $w_{0}$ for range of potential depths $V_{0}$. The data points have been calculated from $((9.61))$ by a numerical estimate of the surface gravity $\bar{g}_{H}$. The solid curves are given by the estimate from (9.16).

chapter.

\subsection{Progress towards an experimental implementation}

To date, investigations of acoustic black holes have primarily focused on the advancement of the theory. However, there has been impressive progress in the experimental manipulation of ultracold atoms, as exemplified by recent experiments with rotating condensates, atoms in optical lattices, low dimensional systems and atom chip traps. It therefore seems likely that further improvements in technology will mean that acoustic black holes may be experimentally realised in BECs in the near future. We briefly report on progress on experiments in BECs, which may have a practical application in forming acoustic black holes in the laboratory, and for measuring their properties. In particular, we consider techniques that are relevant for the implementation of the QdLN.

\subsubsection{Toroidal geometries}

The experimental realisation of a toroidal trap geometry for ultracold atoms, first demonstrated by using two current-carrying loops [231], has been subsequently improved using magnetic waveguides [232], a microchip trap [233] and a four loop configuration [234]. The primary motivation for these experiments is the possibility of performing Sagnac atom interferometry.

Additionally, there have been proposals for generating persistent currents in toroidal geometries by either adiabatic stirring using a rotating paddle (as formed by an optical potential) [230], 
or the method of phase imprinting [235].

\subsubsection{Measurement techniques}

The detection of Hawking radiation in analogue systems may require the development of new measurement techniques. In particular, we note the following proposals that may prove relevant to this cause:

- Schutzhold [236] has proposed a method of detecting phonons based on Raman transitions whereby Bogoliubov excitations of one atomic species are transferred to single particle excitations of another atomic species, which can then be counted using fluorescence measurements.

- Modugno et al. [208] have proposed a scheme for detecting phonons of a condensate in a toroidal geometry, which is based on parametric excitation of excitations via periodic modulation of the confining potential. The resulting pattern formation should be visible and lead to a determination of the Bogoliubov excitation spectrum and quantised circulation. 


\section{Chapter 10}

\section{Stability of the quantum de Laval nozzle and the Hawking effect}

In this chapter we investigate the stability of the quantum de Laval nozzle, which we introduced in the previous chapter. The linear excitations of the system are found by solving the Bogoliubovde Gennes equations. We find both stable and unstable configurations, the latter case exhibiting complex eigenvalues in the excitation spectrum. Following Leonhardt et al. [61], we construct normalisable modes for the dynamically unstable modes and can therefore utilise the classical field method to investigate system dynamics. The connection is made with the Hawking effect, and in particular, we discuss our results with respect to previous investigations of acoustic black holes in fluid models.

\subsection{Bogoliubov theory}

\subsubsection{The Bogoliubov-de Gennes equations}

The linear excitations of the condensate are described by the Bogoliubov-de Gennes (BdG) equations, which are derived from the classical equations of motion for the order parameter. Our discussion follows the references $[213,237,238]$. Consider a solution with small oscillations around a stationary state

$$
\psi(x, t)=e^{-i \mu t / \hbar}\left(\phi_{0}(x)+\sum_{i}\left[u_{i}(x) \beta_{i} e^{-i \omega_{i} t}+v_{i}^{*}(x) \beta_{i}^{*} e^{i \omega_{i} t}\right]\right)
$$

where $\beta_{i}$ and $\beta_{i}^{*}$ are the amplitudes for the oscillations (for the quantum field, these quantities are replaced by the bosonic annihilation and creation operators for the excitations, given by $\hat{b}_{i}$ and $\hat{b}_{i}^{\dagger}$ respectively) and where $\phi_{0}(x)$ is the solution to the time-independent Gross-Pitaevskii equation (GPE) given by (9.19). Substituting this form into the time-dependent GPE and only keeping terms linear in $u_{i}(x)$ and $v_{i}(x)$ yields the familiar BdG equations

$$
\begin{array}{r}
\left(\mathcal{L}_{\mathrm{GP}}-\mu+U_{1 \mathrm{D}}\left|\phi_{0}\right|^{2}\right) u_{i}(x)+U_{1 \mathrm{D}} \phi_{0}^{2} v_{i}(x)=\hbar \omega_{i} u_{i}(x) \\
-U_{1 \mathrm{D}} \phi_{0}^{* 2} u_{i}(x)-\left(\mathcal{L}_{\mathrm{GP}}-\mu+U_{1 \mathrm{D}}\left|\phi_{0}\right|^{2}\right)^{*} v_{i}(x)=\hbar \omega_{i} v_{i}(x)
\end{array}
$$


We have introduced the Gross-Pitaevskii operator

$$
\mathcal{L}_{\mathrm{GP}} \equiv-\frac{\hbar^{2} \partial_{x}^{2}}{2 m}+V(x)+U_{1 \mathrm{D}}\left|\phi_{0}\right|^{2}
$$

with $\left(\mathcal{L}_{\mathrm{GP}}-\mu\right) \phi_{0}=0$. The orthogonality and symmetry relations are chosen by the requirement that the many body Hamiltonian for the interacting Bose gas is diagonal (to quadratic order) in the quasiparticle operators. These are

$$
\begin{aligned}
\int d x\left(u_{i}^{*}(x) u_{j}(x)-v_{i}^{*}(x) v_{j}(x)\right) & =\delta_{i j} \\
\int d x\left(u_{i}(x) v_{j}(x)-v_{i}(x) u_{j}(x)\right) & =0 \\
\int d x\left(u_{i}(x) \phi_{0}^{*}(x)+v_{j}(x) \phi_{0}(x)\right) & =0
\end{aligned}
$$

\section{Orthogonal excitations}

In the form (10.2) there is no guarantee that the mode functions are orthogonal to the condensate wavefunction $\phi_{0}$. This constraint can be imposed by the introduction of the projectors

$$
\begin{array}{r}
\hat{Q}=1-\left|\phi_{0}\right\rangle\left\langle\phi_{0}\right| \\
\hat{Q}^{*}=1-\left|\phi_{0}^{*}\right\rangle\left\langle\phi_{0}^{*}\right|
\end{array}
$$

The excitations orthogonal to the condensate then have the mode functions [238]

$$
\begin{gathered}
\bar{u}_{i}(x)=\hat{Q} u_{i}(x)=u_{i}(x)-c_{i} \phi_{0} \\
\bar{v}_{i}(x)=\hat{Q}^{*} v_{i}(x)=v_{i}(x)+c_{i} \phi_{0}^{*}
\end{gathered}
$$

In position space, and using (10.6), the constants $c_{i}$ are given equivalently by the integrals

$$
c_{i}=\int d x^{\prime} \phi_{0}^{*}\left(x^{\prime}\right) u_{i}\left(x^{\prime}\right)=-\int d x^{\prime} \phi_{0}\left(x^{\prime}\right) v_{i}\left(x^{\prime}\right)
$$

This leads to the so-called modified BdG equations

$$
\begin{array}{r}
\left(\mathcal{L}_{\mathrm{GP}}-\mu+U_{1 \mathrm{D}}\left|\phi_{0}\right|^{2}\right) \bar{u}_{i}(x)+U_{1 \mathrm{D}} \phi_{0}^{2} \bar{v}_{i}(x)=\hbar \omega_{i}\left(\bar{u}_{i}(x)+c_{i} \phi_{0}\right) \\
-U_{1 \mathrm{D}} \phi_{0}^{* 2} \bar{u}_{i}(x)-\left(\mathcal{L}_{\mathrm{GP}}-\mu+U_{1 \mathrm{D}}\left|\phi_{0}\right|^{2}\right)^{*} \bar{v}_{i}(x)=\hbar \omega_{i}\left(\bar{v}_{i}(x)-c_{i} \phi_{0}^{*}\right)
\end{array}
$$

Applying the projectors (10.7) and (10.8) appropriately, noting that $Q^{2}=Q$ for the projectors, and writing the equations in matrix form gives

$$
\mathcal{L}\left(\begin{array}{c}
\bar{u}_{i}(x) \\
\bar{v}_{i}(x)
\end{array}\right)=\epsilon_{i}\left(\begin{array}{c}
\bar{u}_{i}(x) \\
\bar{v}_{i}(x)
\end{array}\right)
$$


where the operator $\mathcal{L}$ is given by [237]

$$
\mathcal{L} \equiv\left(\begin{array}{cc}
\mathcal{L}_{\mathrm{GP}}-\mu+U_{1 \mathrm{D}} \hat{Q}\left|\phi_{0}\right|^{2} \hat{Q} & U_{1 \mathrm{D}} \hat{Q} \phi_{0}^{2} \hat{Q}^{*} \\
-U_{1 \mathrm{D}} \hat{Q}^{*} \phi_{0}^{* 2} \hat{Q} & -\left(\mathcal{L}_{\mathrm{GP}}-\mu+U_{1 \mathrm{D}} \hat{Q}\left|\phi_{0}\right|^{2} \hat{Q}\right)^{*}
\end{array}\right)
$$

We have made use of the fact that $\mathcal{L}_{\mathrm{GP}}$ is Hermitian. The solutions to this equation are the eigenvalues $\epsilon_{i}$ and normal modes $\bar{u}_{i}(x)$ and $\bar{v}_{i}(x)$ of the system.

\section{Properties of $\mathcal{L}$ and dynamical stability}

While $\mathcal{L}$ is not itself Hermitian, it does exhibit certain symmetries that allow us to remark on its eigenvalue spectrum. The operator $\mathcal{L}$ has the following properties [237]

$$
\begin{aligned}
\sigma_{x} \mathcal{L} \sigma_{x} & =-\mathcal{L}^{*} \\
\sigma_{z} \mathcal{L} \sigma_{z} & =\mathcal{L}^{\dagger}
\end{aligned}
$$

in terms of the familiar Pauli matrices

$$
\sigma_{x}=\left(\begin{array}{cc}
0 & 1 \\
1 & 0
\end{array}\right), \quad \sigma_{z}=\left(\begin{array}{cc}
1 & 0 \\
0 & -1
\end{array}\right)
$$

These properties can be used to check the accuracy of the construction of $\mathcal{L}$ when we solve the BdG equations numerically for our analogue model. The property (10.17) implies that $\epsilon_{k}^{*}$ is also an eigenvalue of $\mathcal{L}^{1}$, so that the usual condition for dynamical stability, given by

$$
\operatorname{Im}\left(\epsilon_{i}\right) \leq 0 \text { for all } i,
$$

can be reformulated as the condition

$$
\operatorname{Im}\left(\epsilon_{i}\right)=0 \text { for all } i
$$

From (10.2) it is straightforward to show that [29]

$$
\left(\epsilon_{i}-\epsilon_{i}^{*}\right) \int d x\left(\left|\bar{u}_{i}(x)\right|^{2}-\left|\bar{v}_{i}(x)\right|^{2}\right)=0
$$

That is, a mode $\left(\bar{u}_{i}(x), \bar{v}_{i}(x)\right)$ is normalisable according to (10.4) only if its corresponding eigenvalue $\epsilon_{i}$ is real. If this is true for all modes, the many body Hamiltonian (1.1) takes the diagonal form to quadratic order in the quasiparticle operators [29,226]

$$
H_{\mathrm{Bog}}=E_{0}+\sum_{i} \epsilon_{i}\left(\hat{b}_{i}^{\dagger} \hat{b}_{i}-\int d x\left|\bar{v}_{i}(x)\right|^{2}\right)
$$

corresponding to a set of non-interacting quasiparticle modes.

\footnotetext{
${ }^{1}$ This fact can also be seen directly from taking the complex conjugate of (10.2) while noting that the quantities $U_{1 \mathrm{D}}$ and $V(x)$ are real here.
} 


\section{Landau instability}

Consider a homogeneous condensate with a persistent current. The stationary state, which is a solution to the GPE, is given by $\phi_{0}=\sqrt{n_{0}} e^{i q x}$ for a quasimomentum $q$. As seen from appendix $\mathrm{C}$, the excitation spectrum is Doppler shifted to yield

$$
\epsilon_{k}=\frac{\hbar^{2} k q}{m} \pm \sqrt{\frac{\hbar^{2}}{2 m} k^{2}\left(\frac{\hbar^{2}}{2 m} k^{2}+2 U_{0} n_{0}\right)}
$$

where we have labeled each plane-wave mode by its momentum $k$. Noting that the corresponding flow velocity is $v=\hbar \partial_{x} \theta / m=\hbar q / m$, the condition for all eigenvalues $\epsilon_{p}$ to be non-negative is $v<c$ where $c$ is the speed of sound. Thus is the usual Landau criterion for energetic stability with $v=c$ being the critical velocity [239-241]. When $v>c$, the low momenta modes acquire negative eigenvalues, and the system becomes energetically unstable with respect to these modes; the system can lower its energy by creating quasiparticle excitations of negative energy in the presence of dissipation. In this case the system is thermodynamically unstable (or energetically unstable).

It is worth noting (as discussed also in [149] and references within) that the timescale for dissipation via this mechanism can be made long at low temperatures. When the system is perturbed by an inhomogeneous potential, dynamical instabilities may also be present - this will lead to dramatic growth of the unstable modes, by depleting the condensate. This effect will be the focus of our analysis.

\section{Normalisable modes for dynamical instabilities}

The operator $\mathcal{L}(10.15)$ is not Hermitian and can therefore exhibit complex eigenvalues, which are associated with dynamical instabilities; these are distinct from the energetic (ie. Landau) instabilities arising from real negative eigenvalues. An immediate consequence of (10.21) is that the Bogoliubov modes with complex eigenvalues have zero norm according to the standard construction, and therefore cannot be associated with bosonic operators in the field expansion. However it is still possible to construct normalisable modes using the prescription from Leonhardt et al. [61] (see also [149]), which we now outline. As we shall see, these new modes do correspond to well defined bosonic modes.

In the present discussion, we only consider dynamically unstable modes - ie. modes with complex eigenvalues. It is useful to employ Dirac notation. In particular, the right eigenvector of the operator $\mathcal{L}$, associated with the eigenvalue $\epsilon_{j}$, is defined by

$$
\left|z_{j}^{R}\right\rangle \equiv\left(\begin{array}{c}
u_{j}^{R}(x) \\
v_{j}^{R}(x)
\end{array}\right)
$$

which is a solution to

$$
\mathcal{L}\left|z_{j}^{R}\right\rangle=\epsilon_{j}\left|z_{j}^{R}\right\rangle
$$


Note that for clarity we have dropped the bar notation employed earlier, although it implicitly assumed henceforth that the mode functions are those from (10.9) and (10.10), which are orthogonal to the condensate. Using property (10.16) leads to the expression

$$
\mathcal{L}\left(\sigma_{x}\left|z_{j}^{R}\right\rangle\right)^{*}=-\epsilon_{j}^{*}\left(\sigma_{x}\left|z_{j}^{R}\right\rangle\right)^{*}
$$

Similarly, the left eigenvector of $\mathcal{L}$ is defined as

$$
\left|z_{j}^{L}\right\rangle \equiv\left(\begin{array}{c}
u_{i}^{L}(x) \\
v_{i}^{L}(x)
\end{array}\right)
$$

with

$$
\left\langle z_{i}^{L}\right| \mathcal{L}=\epsilon_{i}\left\langle z_{i}^{L}\right|
$$

using property (10.17) leads to

$$
\mathcal{L}\left(\sigma_{z}\left|z_{i}^{L}\right\rangle\right)=\epsilon_{i}^{*}\left(\sigma_{z}\left|z_{i}^{L}\right\rangle\right)
$$

and additionally using property (10.16) gives

$$
\mathcal{L}\left(\sigma_{x} \sigma_{z}\left|z_{i}^{L}\right\rangle\right)^{*}=-\epsilon_{i}\left(\sigma_{x} \sigma_{z}\left|z_{i}^{L}\right\rangle\right)^{*}
$$

Evidently, the BdG eigenvalues of the system occur in the conjugate pairs $\left(\epsilon_{i}, \epsilon_{i}^{*}\right)$ or $\left(-\epsilon_{i},-\epsilon_{i}^{*}\right)$, which is a consequence of the fact that the many body Hamiltonian (1.1) is Hermitian. We can construct normalisable modes as linear combinations of the above eigenvectors as follows:

$$
\left|\mathcal{Z}_{i}\right\rangle^{+}=\frac{1}{\sqrt{2}}\left[\left|z_{i}^{R}\right\rangle+\sigma_{z}\left|z_{i}^{L}\right\rangle\right]
$$

and

$$
\left.\left|\mathcal{Z}_{i}\right\rangle^{-}=\frac{1}{\sqrt{2}}\left[\left(\sigma_{x} \sigma_{z}\left|z_{i}^{L}\right\rangle\right)^{*}-\left(\sigma_{x} \mid z_{i}^{R}\right)^{*}\right\rangle\right]
$$

with

$$
\left|\mathcal{Z}_{i}\right\rangle^{ \pm} \equiv\left(\begin{array}{c}
\mathcal{U}_{i}^{ \pm}(x) \\
\mathcal{V}_{i}^{ \pm}(x)
\end{array}\right)
$$

To determine the normalisation of such modes, we first note using (10.25) and (10.28) leads to the result

$$
\left(\epsilon_{i}-\epsilon_{j}\right)\left\langle z_{i}^{L} \mid z_{j}^{R}\right\rangle=0
$$

This means we can choose $\left\langle z_{i}^{L} \mid z_{j}^{R}\right\rangle= \pm \delta_{i j}$. Moreover, providing we choose modes with $\left\langle z_{i}^{L} \mid z_{j}^{R}\right\rangle=\delta_{i j}$, the new modes $\left|\mathcal{Z}_{i}\right\rangle^{ \pm}$have positive norm. That is, they satisfy the normalisa- 
tion conditions

$$
\int d x\left(U_{i}^{ \pm *}(x) U_{j}^{ \pm}(x)-V_{i}^{ \pm *}(x) V_{j}^{ \pm}(x)\right)=\delta_{i j}
$$

and

$$
\int d x\left(U_{i}^{ \pm}(x) V_{j}^{ \pm}(x)-V_{i}^{ \pm}(x) U_{j}^{ \pm}(x)\right)=0
$$

which are of the same form as the (10.4) and (10.5) given previously for the dynamically stable modes. The resulting modes (10.31) and (10.32) are thus normalisable, and moreover, taking these modes with the set of modes for dynamically stable modes forms a complete orthonormal basis; this is a direct consequence of the fact that (10.31) and (10.32) are linear superpositions of modes that are orthonormal to the dynamically stable modes.

The creation and annihilation operators for the new modes satisfy the commutation relations for bosonic operators, but show up as non-diagonal terms in the Hamiltonian subspace for the dynamically unstable modes as [61]

$$
\begin{aligned}
& \hat{H}_{2}=\sum_{j} \operatorname{Re}\left(\epsilon_{j}\right)\left[\left(\hat{b}_{j+}^{\dagger} \hat{b}_{j+}-\hat{b}_{j-}^{\dagger} \hat{b}_{j-}\right)-\int d x\left(\left|V_{j}^{+}\right|^{2}-\left|V_{j}^{-}\right|^{2}\right)\right] \\
& +\sum_{j} i \operatorname{Im}\left(\epsilon_{j}\right)\left[\left(\hat{b}_{j+} \hat{b}_{j-}-\hat{b}_{j+}^{\dagger} \hat{b}_{j-}^{\dagger}\right)+\int d x\left(U_{j}^{+} V_{j}^{-}-U_{j}^{+*} V_{j}^{-*}\right)\right]
\end{aligned}
$$

where the sum is taken over only the dynamically unstable modes, and where $\hat{b}_{j \pm}$ is the annihilation operator and $\hat{b}_{j \pm}^{\dagger}$ the creation operator corresponding to the mode $\left|\mathcal{Z}_{j}\right\rangle^{ \pm}$. Dynamically unstable modes are therefore associated with non-degenerate parametric amplification [33], which leads to growth in the unstable modes at the expense of the condensate mode. For short time dynamics, the complex eigenvalue will generate exponential growth in each unstable mode. It is this effect that has been suggested to provide the closest analogy with the Hawking effect for BECs [61, 149]. However, this picture neglects higher order interactions that may be present in the full Hamiltonian, and therefore is likely to fail for dynamics on long time scales. We will investigate this further in section 10.3.

\subsection{Spectrum of the QdLN}

Having now established the theory of elementary excitations for a BEC, we use this to find the excitation spectrum of the QdLN, using the stationary transonic solutions found in the preceding chapter.

\subsubsection{Numerical calculation of Bogoliubov modes}

For the case of the QdLN the excitation spectrum and modes must be found by solving (10.14). To implement this numerically, we use the dimensionless parameters introduced in section 9.4.4. 

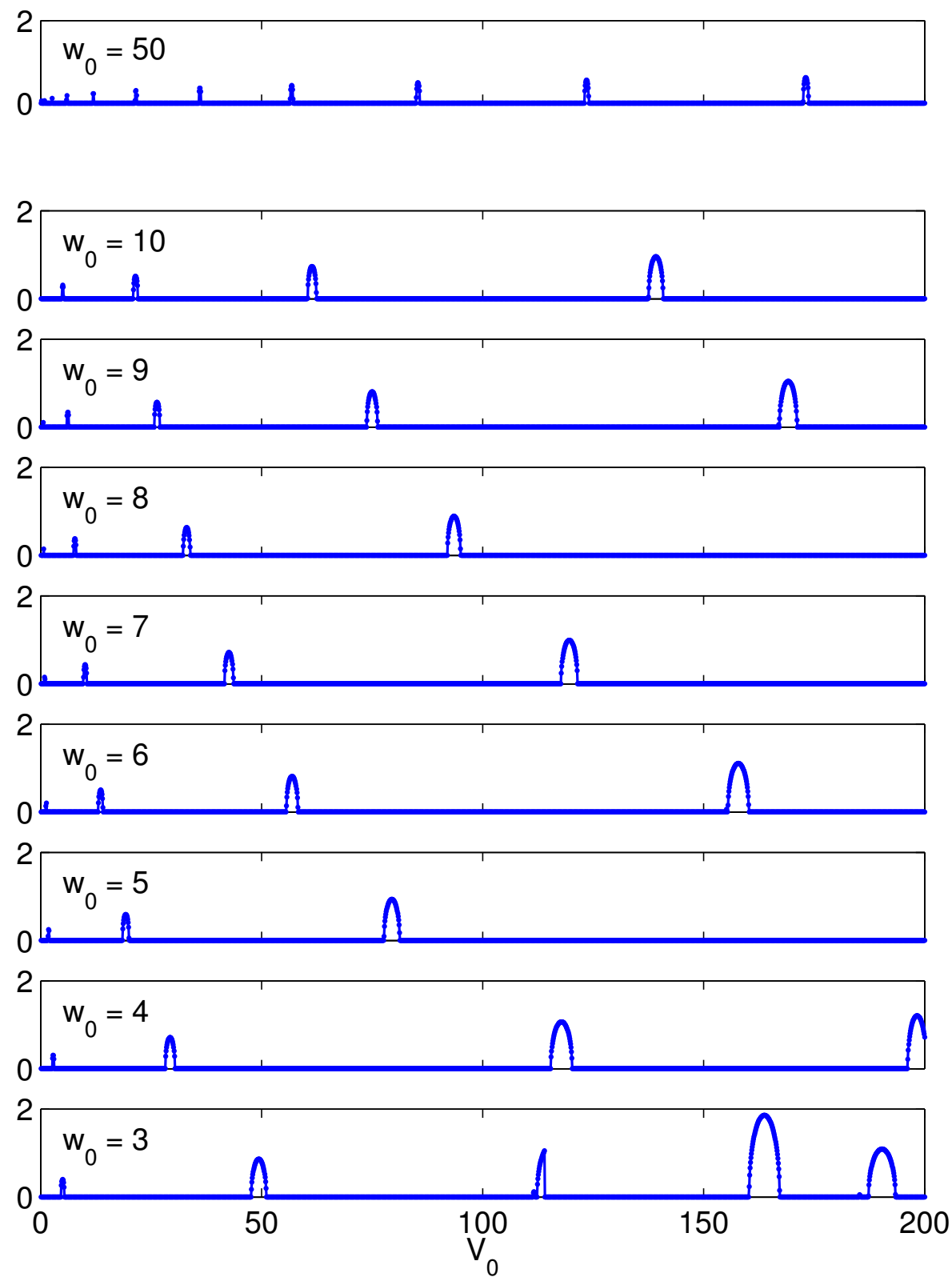

Figure 10.1: Stability diagram of quantum de Laval nozzle: Largest imaginary component of eigenvalue for the Bogoliubov modes for a range of winding numbers as a function of $V_{0}$. The values of the imaginary eigenvalue component and $V_{0}$ are both given in the dimensionless units introduced in section 9.4.4. Each solution was found using a spatial grid of 256 points.

In these units the BdG operator (10.15) becomes

$$
\overline{\mathcal{L}}=\left(\begin{array}{cc}
\overline{\mathcal{L}}_{\mathrm{GP}}-\bar{\mu}+C \hat{Q}\left|\phi_{0}\right|^{2} \hat{Q} & C \hat{Q} \phi_{0}^{2} \hat{Q}^{*} \\
-C \hat{Q}^{*} \phi_{0}^{* 2} \hat{Q} & -\left(\overline{\mathcal{L}}_{\mathrm{GP}}-\bar{\mu}+C \hat{Q}\left|\phi_{0}\right|^{2} \hat{Q}\right)^{*}
\end{array}\right)
$$


with the Gross-Pitaevskii operator

$$
\overline{\mathcal{L}}_{\mathrm{GP}} \equiv-\frac{1}{2} \partial_{\bar{x}}^{2}+\bar{V}_{0} \cos ^{2}(2 \pi \bar{x})+C\left|\phi_{0}\right|^{2}
$$

where $\phi_{0}$ is the stationary transonic solution for the QdLN, which has been renormalised to a single-particle wavefunction. The dimensionless eigenvalue of the $\mathrm{BdG}$ operator is given by $\overline{\epsilon_{i}}=$ $\kappa \epsilon_{i}$. Henceforth we drop the bar notation.

The diagonalisation of (10.38) can be carried out in a plane wave basis by calculating the corresponding matrix elements for each term in (10.14). This procedure must be performed for each set of parameters $V_{0}$ and $w_{0}$. To perform the diagonalisation for the results presented here we used the eig.m function from Matlab. The numerical accuracy of the construction of the $\mathcal{L}$ operator was also checked by using the symmetry properties (10.16) and (10.17). For a dynamically unstable configuration, we construct normalisable modes using the procedure outlined in section 10.1.1. We additionally sort the eigenvalues in ascending order by $\operatorname{Re}\left(\epsilon_{i}\right)$ and label the modes accordingly.

\subsubsection{Results}

Figure 10.1 shows the stability diagram for the QdLN that results by performing the diagonalisation for a range of parameters, $V_{0}$ and $w_{0}$. For each point we have calculated the maximum of the absolute value for the imaginary part of all eigenvalues. The essential features we observe are: (i) there are regions exhibiting dynamic instabilities; (ii) these regions become narrower and smaller in magnitude, but more closely spaced for larger values of the winding number $w_{0}$, whereas they become broader and larger in magnitude as the potential depth $V_{0}$ increases.

Figures 10.2 (a)-(d) show the eigenvalue spectrum for the first few modes for four different sets of parameters. In particular: (a) shows the spectrum for $w_{0}=3$ and $V_{0}=140$; (b) shows the spectrum for $w_{0}=3$ and $V_{0}=163.7$; (c) shows the spectrum for $w_{0}=10$ and $V_{0}=$ 100; and (d) shows the spectrum for $w_{0}=10$ and $V_{0}=139.2$. While all cases have negative eigenvalues, indicating energetic (Landau) instabilities due to non-zero flow, only cases (b) and (d) correspond to dynamical instabilities also. Following the theory of the previous section, the onset of a dynamical instability is associated with a pair of modes (labelled by $j$ and $k$ say) with complex eigenvalues that satisfy $\operatorname{Re}\left(\epsilon_{j}\right)=-\operatorname{Re}\left(\epsilon_{k}\right)$. In particular, for the lower winding number $w_{0}=3$, Figure 10.2 (b) indicates that modes 1 and 6 are unstable with $\operatorname{Re}\left(\epsilon_{1}\right)=-\operatorname{Re}\left(\epsilon_{6}\right)$. On the other hand, for the larger winding number $w_{0}=10$, Figure 10.2 (d) indicates that modes 5 and 6 are unstable with $\operatorname{Re}\left(\epsilon_{5}\right)=-\operatorname{Re}\left(\epsilon_{6}\right)$.

We also show the corresponding mode functions $u_{i}(x)$ and $v_{i}(x)$ that result from the solutions of the BdG equations. In particular, Figure 10.3 shows the mode functions for $w_{0}=3$ and $V_{0}=163.7$ and (d) Figure 10.4 shows the mode functions for $w_{0}=10$ and $V_{0}=139.2$, both which have dynamical instabilities. We further note, although not shown here, that for $w_{0}=3$, the mode functions for $V_{0}=140$ (dynamically stable) are very similar to the $V_{0}=163.7$ case (dynamically unstable), and likewise the mode functions for $w_{0}=10$ and $V_{0}=100$ (dynamically stable) are very similar to the $V_{0}=139.2$ case. We have also verified in all cases that for the 


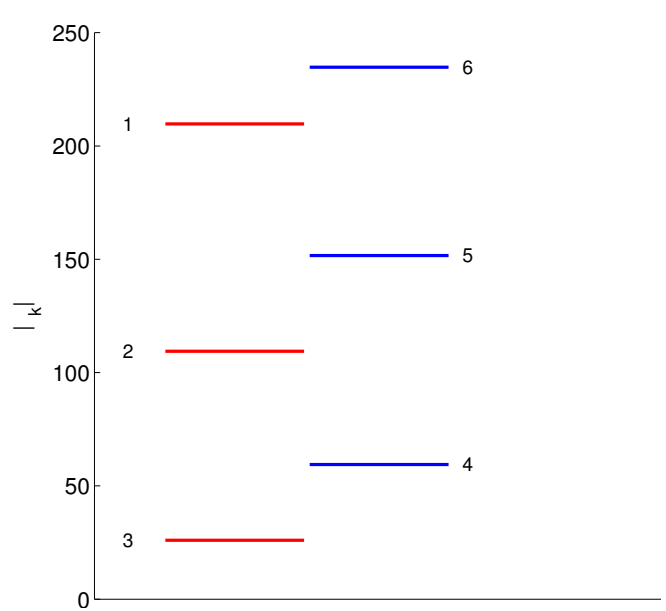

(a) $V_{0}=140, w_{0}=3$

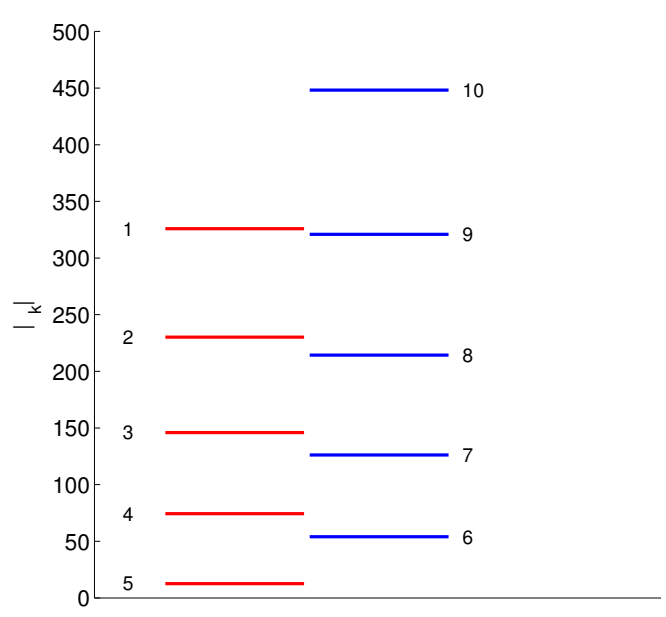

(c) $V_{0}=100, w_{0}=10$

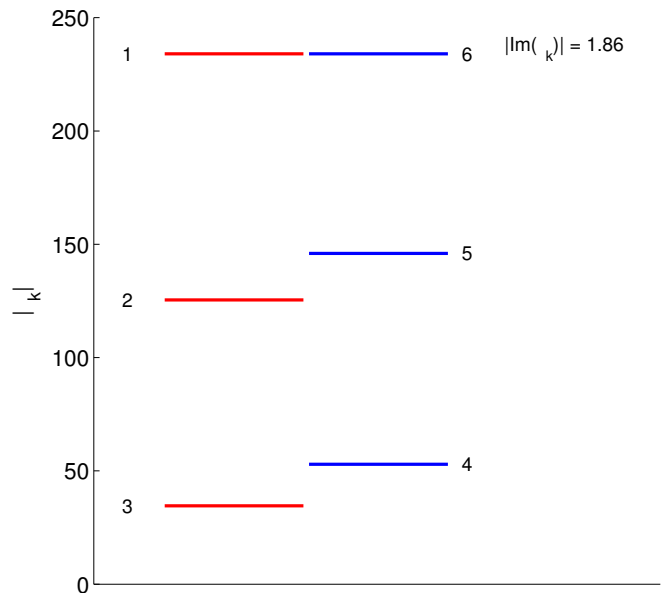

(b) $V_{0}=163.7, w_{0}=3$

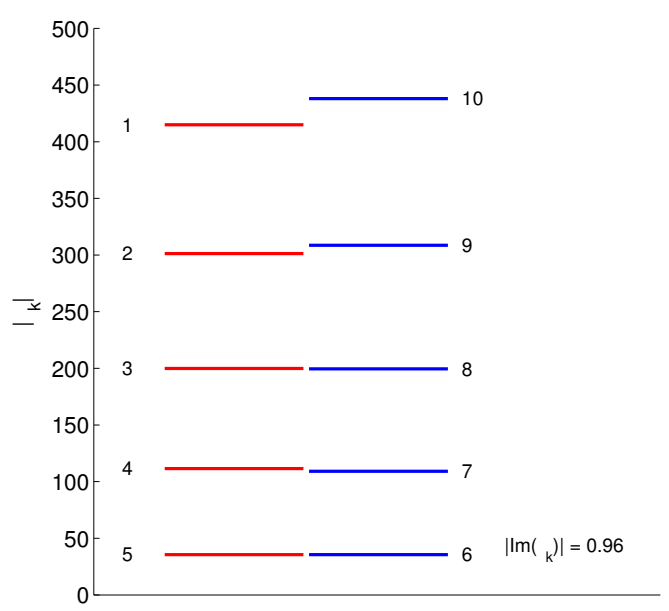

(d) $V_{0}=139.2, w_{0}=10$

Figure 10.2: Eigenvalue spectrum (all negative eigenvalues and same number of lowest positive eigenvalues) of QdLN found from solutions of BdG equations. The red lines show the moduli of the negative eigenvalues and the blue lines show the positive eigenvalues. Eigenvalues have an imaginary component as indicated, only for a pair of eigenvalues that satisfies $\epsilon_{i}=-\epsilon_{j}$ exactly. Solutions have been found using a spatial grid of 1024 points.

high energy modes $\left|u_{i}(x)\right|^{2} \approx 1$ and $\left|v_{i}(x)\right|^{2} \approx 0$. That is, the high energy modes correspond to single particle excitations as expected from Bogoliubov theory. However, the plots show that the mode functions exhibit a rich structure for the low energy modes, not least being the sort of "localisation" of modes that is associated with the acoustic black hole geometries. This is clearly demonstrated in Figure 10.4 for the case where $w_{0}=10$ and $V_{0}=139.2$. In particular, for modes $i \leq 4, u_{i}(x)$ is localised in the region $0 \leq x \leq 0.5$, which corresponds to the supersonic region, whereas $v_{i}(x)$ is localised in the region $-0.5 \leq x \leq 0$, corresponding to the subsonic region. For modes $i \geq 7$ we find the reverse is true in general, although the localisation occurs to a lesser extent. Modes $i=5,6$ (the dynamically unstable modes) indicate a crossover between these two regimes. 

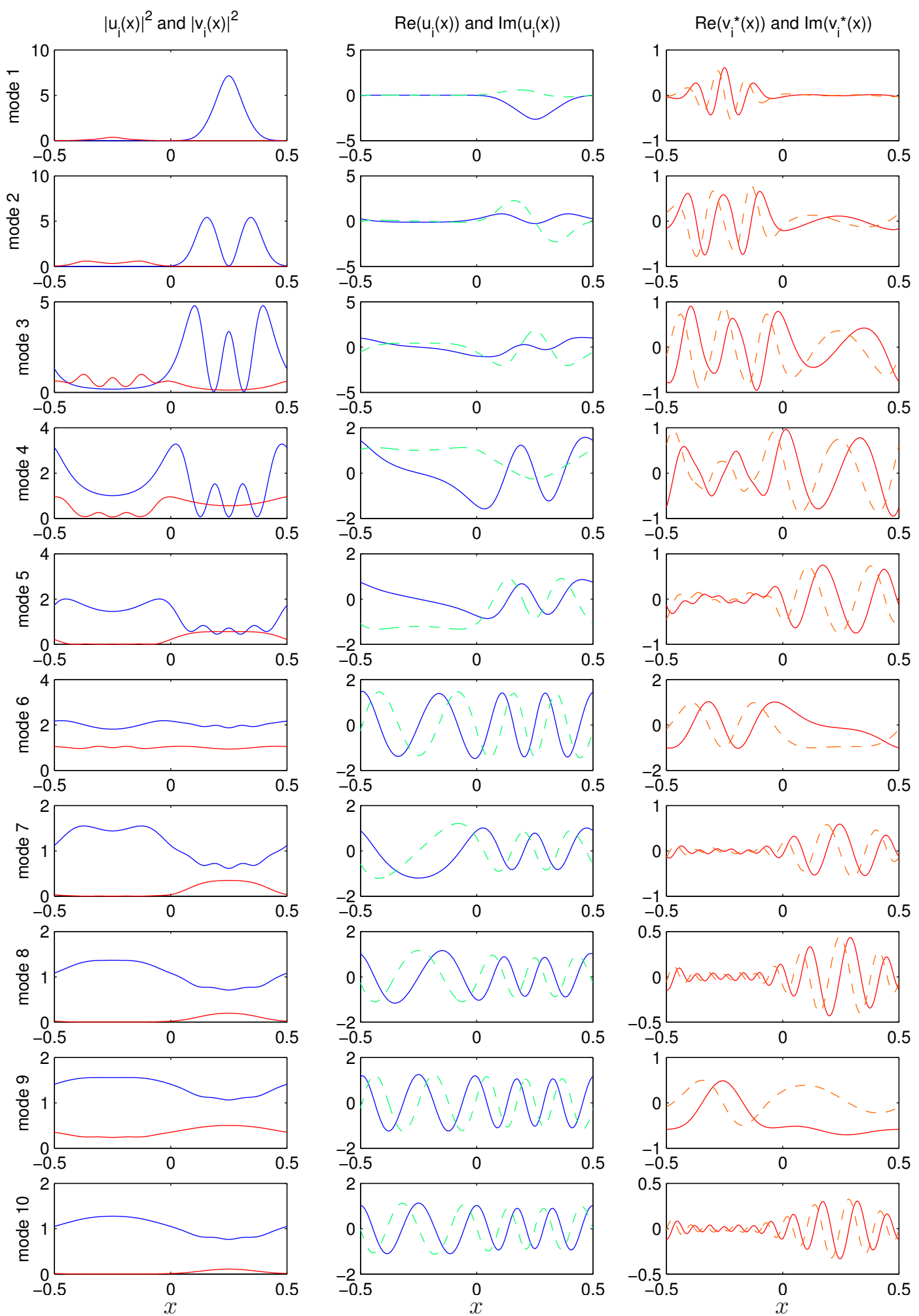

Figure 10.3: Dynamically unstable configuration: Orthogonal mode functions for $V_{0}=163.7$ and $w_{0}=3$. Modes $1 \leq i \leq 10$ are shown. In the first column $\left|u_{i}(x)\right|^{2}$ is given by the blue curve, and $\left|v_{i}(x)\right|^{2}$ is given by the red curve. In the second column $\operatorname{Re}\left(u_{i}(x)\right)$ is given by the solid blue curve, and $\operatorname{Im}\left(v_{i}(x)\right)$ is given by the dashed green curve. In the third column $\operatorname{Re}\left(v_{i}^{*}(x)\right)$ is given by the solid red curve, and $\operatorname{Im}\left(v_{i}^{*}(x)\right)$ is given by the dashed orange curve. Solutions have been found using a spatial grid of 1024 points. 

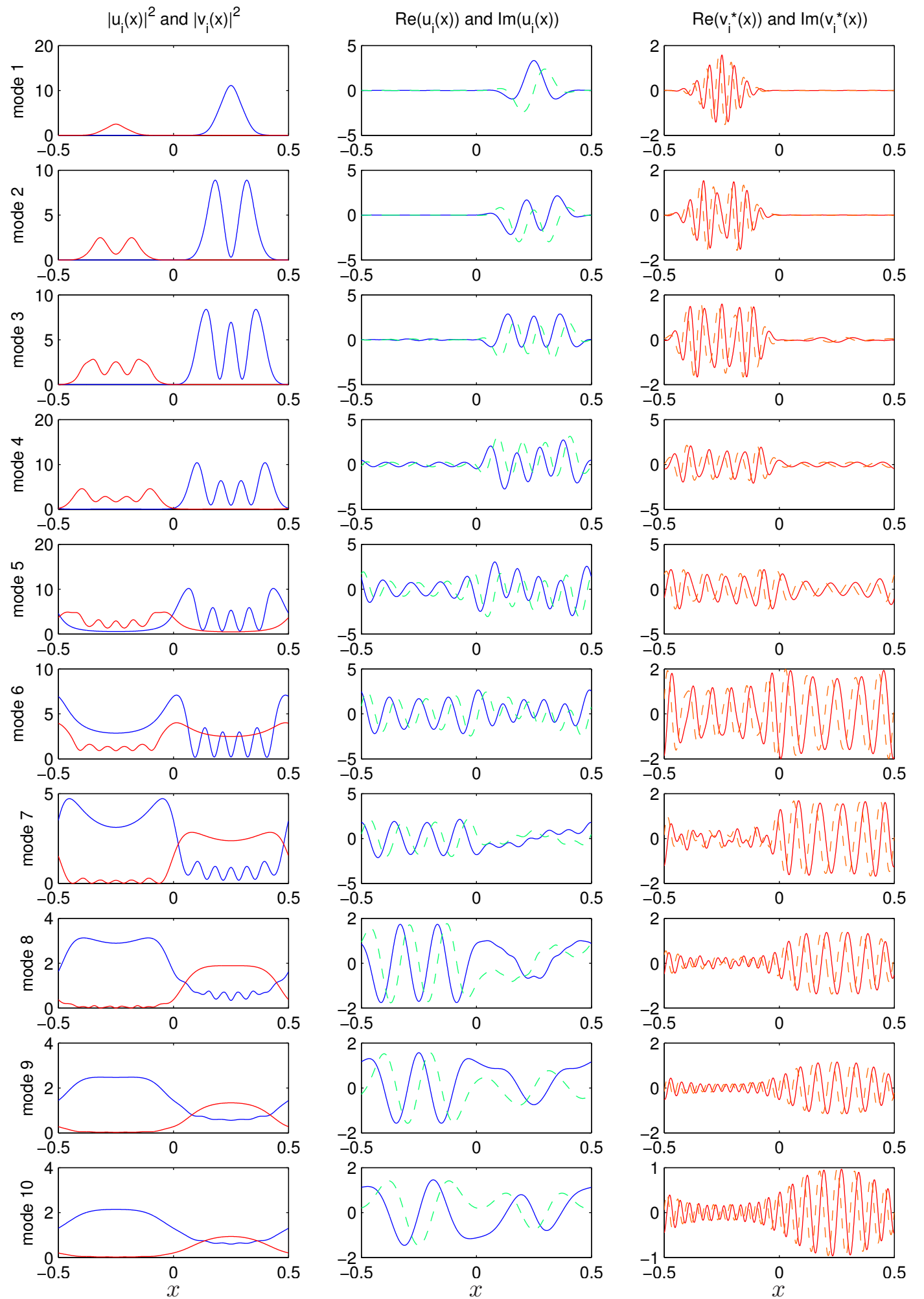

Figure 10.4: Dynamically unstable configuration: Orthogonal mode functions for $V_{0}=139.2$ and $w_{0}=10$. Modes $1 \leq i \leq 10$ are shown. In the first column $\left|u_{i}(x)\right|^{2}$ is given by the blue curve, and $\left|v_{i}(x)\right|^{2}$ is given by the red curve. In the second column $\operatorname{Re}\left(u_{i}(x)\right)$ is given by the solid blue curve, and $\operatorname{Im}\left(v_{i}(x)\right)$ is given by the dashed green curve. In the third column $\operatorname{Re}\left(v_{i}^{*}(x)\right)$ is given by the solid red curve, and $\operatorname{Im}\left(v_{i}^{*}(x)\right)$ is given by the dashed orange curve. Solutions have been found using a spatial grid of 1024 points. 


\subsection{Dynamics}

Thus far, we have found the elementary excitations for the QdLN and found that this indicates dynamically unstable configurations for certain sets of parameters. In order to verify that such configurations do indeed lead to exponential growth in the unstable modes, we must check the dynamics of the system (that is, to check the perturbation theory is valid). To this end we use the classical field method, based on the truncated Wigner approximation (TWA), that was introduced in chapter 1 and further developed in chapter 7. We consider simulations for two different winding numbers, $w_{0}=3$ and $w_{0}=10$, which lead to qualitatively different behaviour for the mode dynamics.

\subsubsection{Truncated Wigner approximation}

The procedure for performing quantum field theory simulations using the TWA requires evolving a multimode classical field (7.2) using the projected GPE (7.5). The initial field is given by

$$
\psi(x, t=0)=\psi_{0}(x)+\sum_{i>0}\left(u_{i}(x) \beta_{i}+v_{i}^{*}(x) \beta_{i}^{*}\right)
$$

where $\psi_{0}(x)$ is a stationary state of the GPE (ie. the transonic solutions of the QdLN), and where $u_{i}$ and $v_{i}$ are the corresponding orthogonal mode functions (recall we have dropped the bar notation) from (10.9) and (10.10). The normalised modes for any dynamical instabilities are constructed as outlined in the section 10.1.1. The complex random variables $\beta_{i}$ satisfy Gaussian statistics given by (7.12) and (7.12) that amount to adding to half a particle per mode to the stationary state - this corresponds to the quasiparticle vacuum.

There is an assumption inherent in this approach: when dynamical instabilities are present the system cannot be in a stationary configuration (although it may be quasi-stationary), and the choice of a quasiparticle vacuum for the initial state may not be valid. However, in practice, the formation of a dynamically unstable QdLN configuration would likely require moving from a stable configuration to an unstable one by moving through parameter space (ie. by changing $w_{0}$ and/or $V_{0}$ ). One possible route to achieving this would be to start with a homogeneous flow in a toroid at the critical velocity $v=c$, and then to switch on the (double de Laval nozzle) potential $V(x)$ by adiabatically ramping $V_{0}$ from zero to its final value. In this case the system should remain in its vacuum state until the dynamical instability is encountered. Moreover, for the parameters considered in this chapter, we have verified that the mode functions for a value of $V_{0}$ close to - but not on - an instability are quantitatively similar to those for the instability itself. This seems to suggest that the quasiparticle vacuum for the dynamically unstable modes should provide a good approximation to the case where the unstable configuration is reached via an adiabatic process. 


\subsubsection{Quasiparticle projection}

The projection into the Bogoliubov basis, found by inverting (10.1), is given by

$$
\beta_{i}(t)=\int d x\left(u_{i}^{*}(x) \psi(x, t)-v_{i}^{*}(x) \psi^{*}(x, t)\right)
$$

The quasiparticle number in each mode is then

$$
N_{i}(t)=\left\langle\beta_{i}^{*}(t) \beta_{i}(t)\right\rangle_{W}-\frac{1}{2}
$$

where the $\langle\cdot\rangle_{W}$ indicates an ensemble average over many TWA trajectories. We note that in some instances, however, when the interpretation is clear, we calculate the quasiparticle numbers using single trajectory simulations.

\subsubsection{Numerical details}

For the evolution of the projected GPE we have used the projected RK4IP algorithm, which is outlined in Appendix B. We employ the dimensionless units introduced in section 9.4. For the simulations presented here we have used the following parameters:

- $N_{0}=10^{6}$, the total number of atoms in the condensate.

- $M=1024$, the number of modes for the system in the low energy subspace of the projector. This requires $n_{x}=2 M=2048$ points for the spatial grid.

This choice of parameters satisfies the criterion $N_{0} \gg M$ for the validity of the TWA (see discussion from section 7.2.2). Unless otherwise stated, for all simulations we chose a time step for the RK4IP algorithm that ensured the change in total field normalisation during each trajectory was $\Delta N / N<10^{-6}$.

Although it would be desirable to further extend our analysis to large winding numbers, there are several difficulties associated with this. In particular, referring to Figure 9.7, the nonlinearity increases with winding number, so that simulations would require decreasing the time step for convergence $^{2}$. Moreover, the Nyquist criterion means that to represent the non-trivial phase variation of the condensate wavefunction requires a spatial grid with $n_{x} \geq 2 w_{0}$ points. An even more stringent requirement will be necessary to represent the structure of the mode functions $u_{i}(x)$ and $v_{i}(x)$.

\subsubsection{Results for $w_{0}=3$}

For a winding number of $w_{0}=3$, the quasiparticle mode occupations have been calculated from single trajectory simulations for two cases: (i) Figure 10.5 shows results for $V_{0}=140$, and (ii) Figure 10.6 shows results for $V_{0}=163.7$. Referring to the results in section 10.2, the first

\footnotetext{
${ }^{2}$ To see this more clearly, consider the homogeneous case with non-zero circulation. In this case the dimensionless chemical potential of the condensate is $\mu=C$, for which the simulation time step must satisfy $\Delta t \ll 1 / C$ to account for the phase variation of the condensate wavefunction.
} 


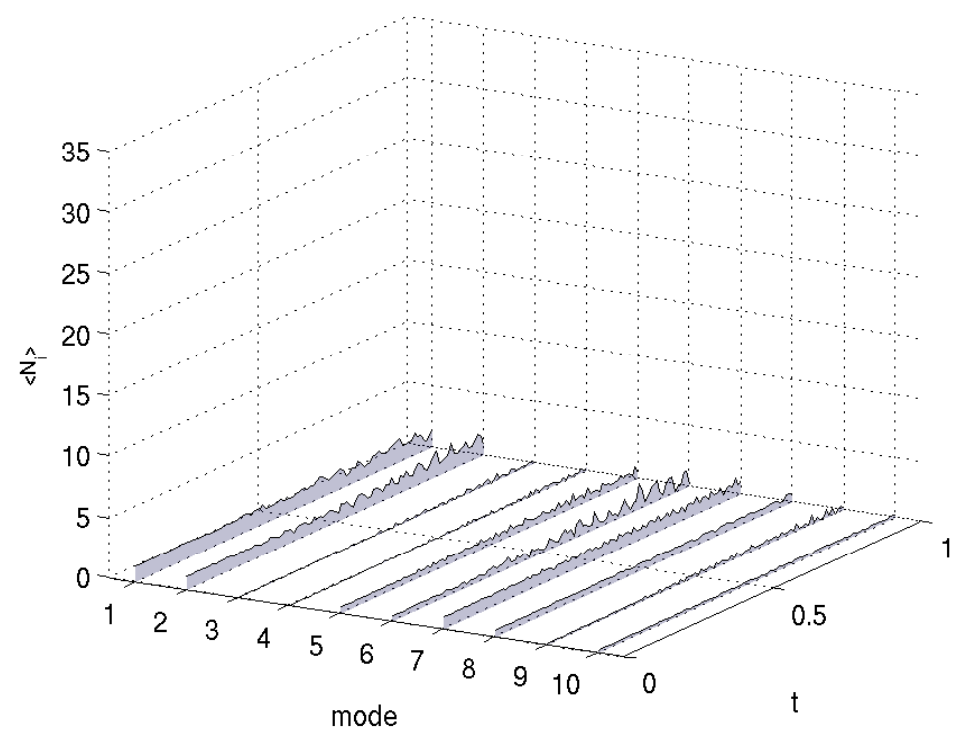

Figure 10.5: Bogoliubov mode populations for single trajectory from TWA evolution for parameters $V_{0}=140, w_{0}=3$, corresponding to a dynamically stable configuration. The simulation end time is $t=1$.

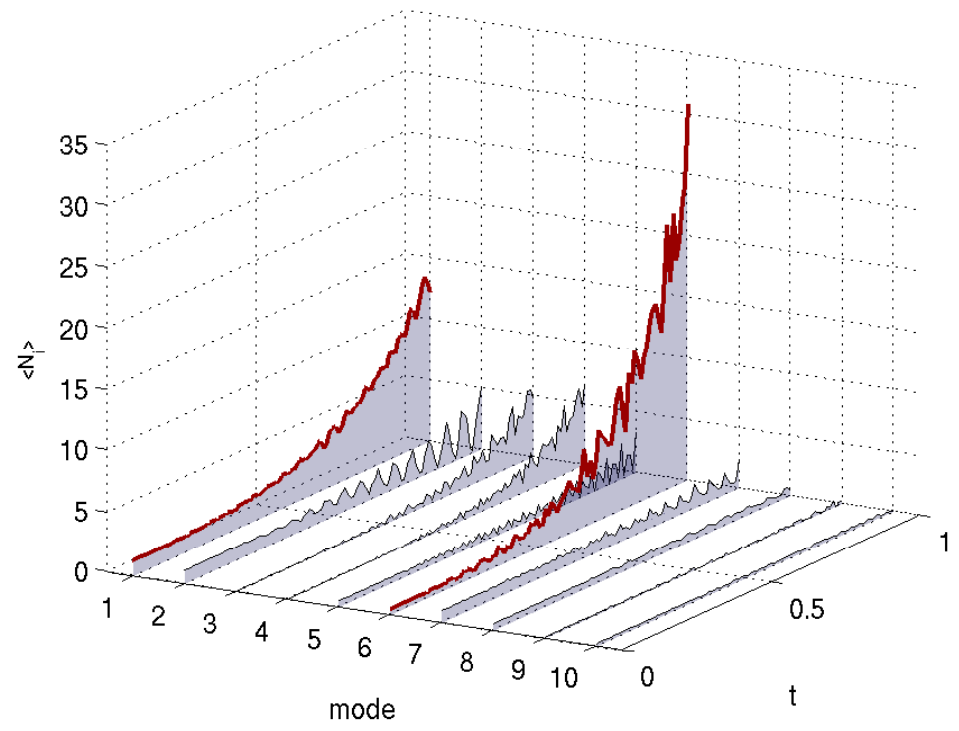

Figure 10.6: Bogoliubov mode populations for single trajectory from TWA evolution for parameters $V_{0}=163.7, w_{0}=3$, corresponding to a dynamically unstable configuration. The simulation end time is $t=1$. The dynamically unstable modes $(i=1,6)$ are indicated by the dark red curves.

case corresponds to a dynamically stable configuration, whereas the second case corresponds to a dynamically unstable configuration. In the latter case, modes $i=1,6$ are unstable, and we observe exponential growth in these modes, which is absent in the stable configuration. The growth is seeded by the Gaussian noise in the initial state. This result confirms the expectation that the 


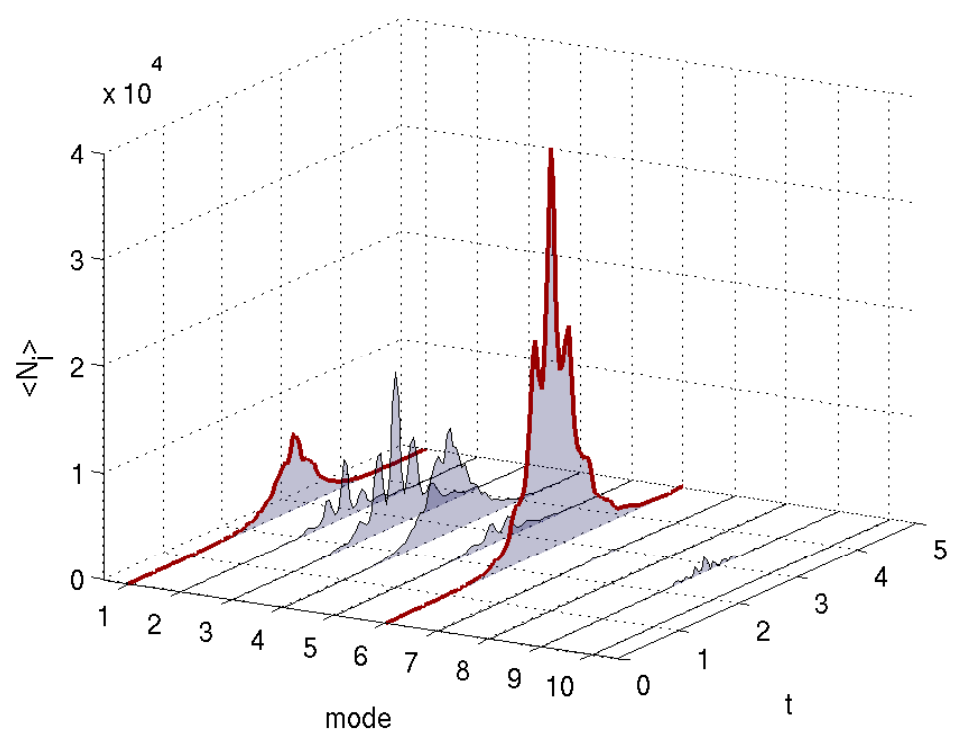

Figure 10.7: Bogoliubov mode populations for single trajectory from TWA evolution for parameters $V_{0}=163.7, w_{0}=3$, corresponding to a dynamically unstable configuration. The simulation end time is $t=5$. The dynamically unstable modes $(i=1,6)$ are indicated by the dark red curves.

mode dynamics for the unstable modes are governed - at short times — by the Hamiltonian (10.37) for a non-degenerate parametric amplifier. Note that there is also some growth in modes $i=2,3,4,5$. We have also checked the occupation numbers for modes $i>10$ and found these to be negligible compared with the modes shown in the plots $i \leq 10$.

We have also investigated the behaviour of the dynamically unstable configuration for longer times. Single trajectory results for a simulation time of $t=5$ are shown in Figure 10.7. Here we observe growth in the unstable modes $(i=1,6)$ until $t \sim 2.75$ where there is a peak in the mode populations, followed by a decay of occupation numbers. There is also significant growth (and decay) in the stable modes $i=2,3,4,5$, although the onset of this growth is delayed when compared with the unstable modes. Therefore the system undergoes a period of excitation followed by an apparent revival of the initial unexcited state. This is also evident in the coordinate space density plots for the same simulation given in Figures 10.8 (a) and (b). In particular, plot (a) shows large scale density fluctuations for $2 \lesssim t \lesssim 3.5$. Plot (b) shows the density relative to the initial state, from which it is clear that the density fluctuations are localised in the region $0 \leq x \leq 0.5$ corresponding to the supersonic region for the system. It seems plausible that such excitations and revivals should continue to repeat, which would result in a "ringing" type excitation of the condensate. However, due to the significant computational time required, we did not check this prediction.

The revival of system is most likely due to nonlinear mode mixing, which is neglected in the non-degenerate parametric amplifier picture. In particular, the back-reaction of quasiparticle modes on the condensate should become significant for large mode occupations. Moreover, the topological constraint imposed by the periodicity of the system (ie. by a fixed winding number) 


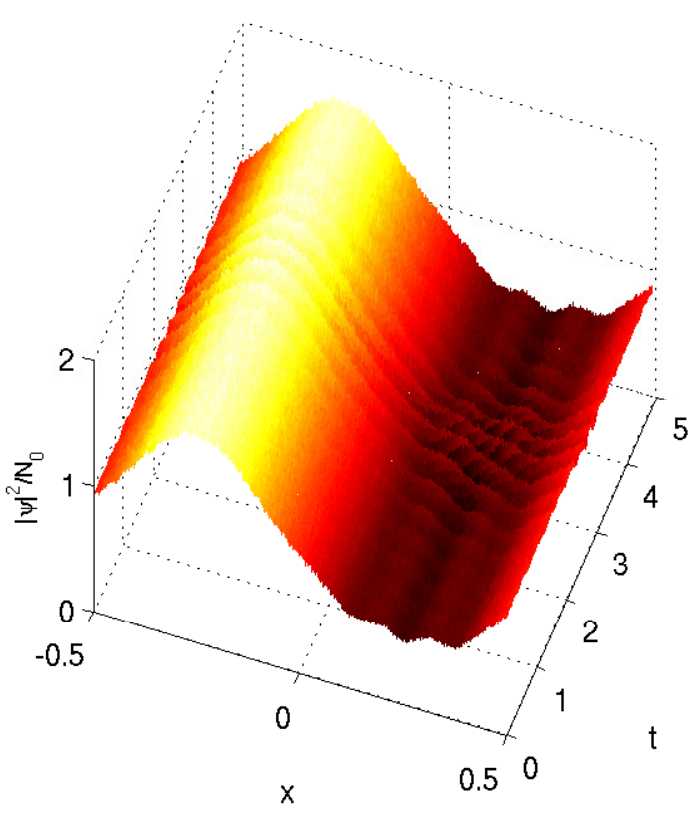

(a)

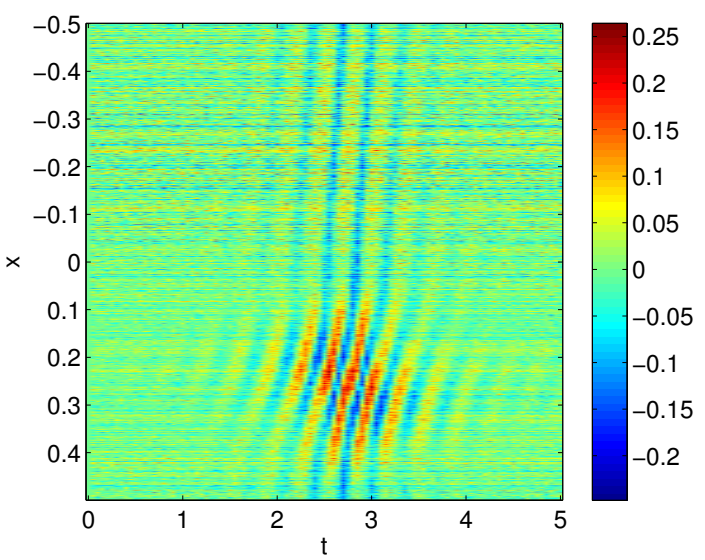

(b)

Figure 10.8: Coordinate space density vs time for unstable configuration with $w_{0}=3$ and $V_{0}=$ 163.7: (a) gives the normalised density of the field by $n(x, t)=|\psi(x, t)|^{2} / N_{0}$; (b) gives a intensity plot of the change in density from the initial state by $\Delta n(x, t)=n(x, t)-n(x, 0)$. Note that in plot (a) the high frequency noise has been filtered out during post-processing of the data.

means that the decay of circulation for the superfluid flow is forbidden. This accounts for the fact that the system cannot reach a quasi-stationary state corresponding to a different value of $w_{0}$, without a corresponding change in $V_{0}$. Indeed such quasiperiodic behaviour is a feature of the Fermi-Pasta-Ulam problem for nonlinear systems, which has been studied previously in BECs [242].

\subsubsection{Results for $w_{0}=10$}

For a winding number $w_{0}=10$, we similarly compare results for two different cases: (i) Figure 10.9 shows results for $V_{0}=100$, and (ii) Figure 10.10 shows results for $V_{0}=139.2$. Referring to the results in section 10.2, the first case corresponds to a dynamically stable configuration, whereas the second case corresponds to a dynamically unstable configuration. In the latter case, modes $i=5,6$ are unstable. Because the single trajectory results exhibit highly oscillatory behaviour for the majority of modes, indicative of nonlinear mode mixing, we have calculated the mode occupations by averaging over 40 trajectories. Moreover, to investigate the short time dynamics we have chosen a simulation time of $t=0.1$.

In contrast to the $w_{0}=3$ case, for $w_{0}=10$ the results for the stable and unstable configurations are quantitatively similar. Here both configurations have significant growth for the populations of modes $i=5,6,13$, as well as modest growth in other modes as shown. Therefore, the results cannot be fully interpreted in terms of non-degenerate parametric amplification of 


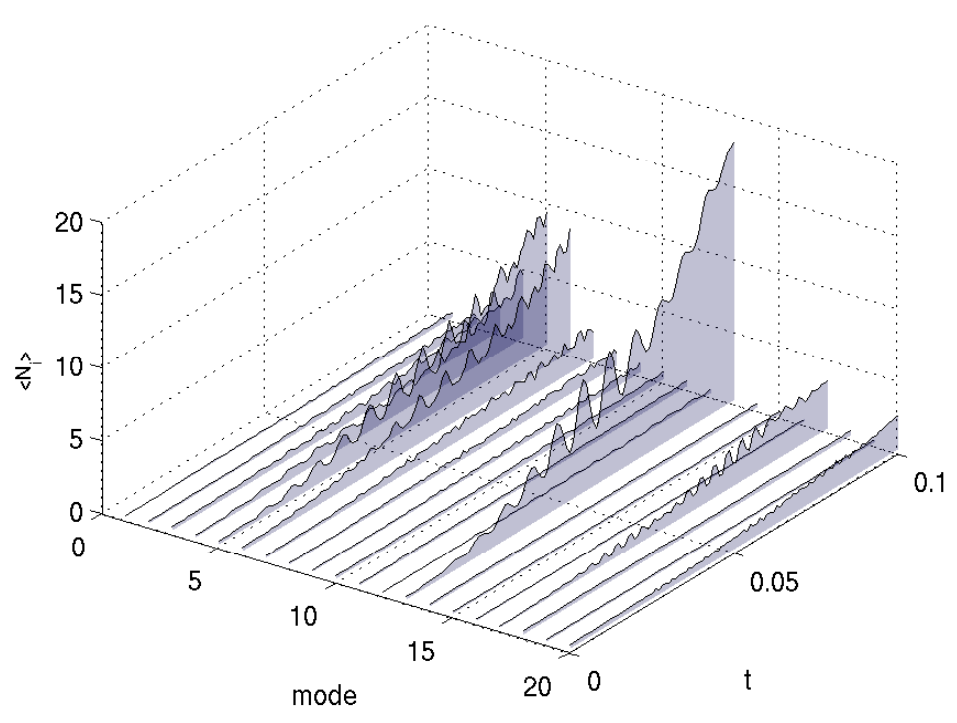

Figure 10.9: Bogoliubov mode populations from TWA evolution for parameters $V_{0}=100, w_{0}=$ 10 , corresponding to a dynamically stable configuration. The simulation end time is $t=0.1$. The quasiparticle number has been calculated by averaging over 40 trajectories.

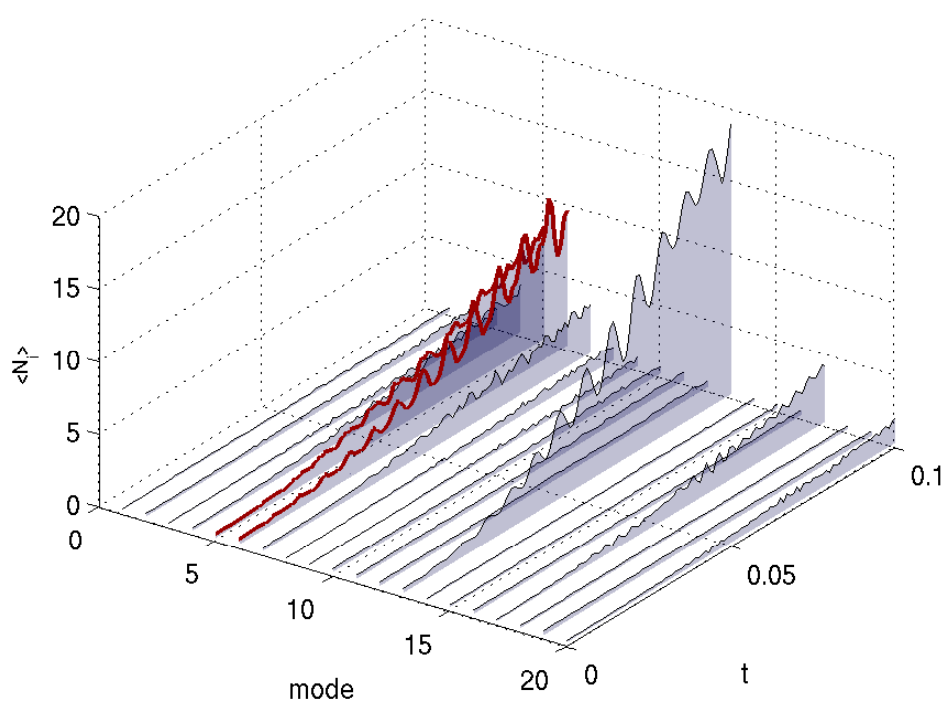

Figure 10.10: Bogoliubov mode populations from TWA evolution for parameters $V_{0}=139.2$, $w_{0}=10$, corresponding to a dynamically unstable configuration. The simulation end time is $t=0.1$. The quasiparticle number has been calculated by averaging over 40 trajectories. The dynamically unstable modes $(i=5,6)$ are indicated by the dark red curves.

the unstable modes. We should emphasise that for the two values of $V_{0}$, the modes $i=5,6$ are dynamically unstable only for $V_{0}=139.2$, and the growth in these same modes for $V_{0}=100$ is therefore unexpected. 


\section{Anomalous mode growth}

Perhaps more significant is the dramatic growth of mode $i=13$, which cannot be accounted for by either Landau or dynamical instabilities, since for that mode the eigenvalue is both real and positive. We will henceforth refer to this as an anomalous mode. It is of some interest to examine the eigenfunctions for this mode, which can be seen in Figure 10.11. A distinctive feature of this mode is immediately apparent: both $u_{13}(x)$ and $v_{13}^{*}(x)$ are approximate plane waves. Specifically, the positive frequency plane wave component for $u_{13}(x)$ is right travelling (with the superfluid flow), whereas the negative frequency plane wave component for $v_{13}(x)$ is left travelling (against the superfluid flow). Moreover, it is evident that $u_{13}(x)$ is blueshifted in the ergoregion $(v>c)$, whereas $v_{13}(x)$ is redshifted in the ergoregion. Also of note, is that modes $i=17,20$ also experience some growth. Referring to Figure 10.11, the mode functions in those cases are also approximately plane waves, although to a lesser extent than for the $i=13$ case, as surmised from the varying density.

To further illuminate the issue we also plot the first 20 eigenvalues for the unstable configuration $\left(V_{0}=139.2\right.$ and $\left.w_{0}=10\right)$ in Figure 10.12. Here we clearly see the relatively large imaginary components for the unstable modes $i=5,6$, and further note that $\operatorname{Re}\left(\epsilon_{5}\right)=-\operatorname{Re}\left(\epsilon_{6}\right)$ as required by the symmetries of the $\mathrm{BdG}$ equations for a dynamical instability. It is interesting to note that modes $i=12,13$ are almost degenerate with $\operatorname{Re}\left(\epsilon_{12}\right) \approx \operatorname{Re}\left(\epsilon_{13}\right)$, which may be evidence that configuration is close to another dynamical instability, and may account for the large growth seen in mode $i=13$. (It should be noted, however, that this is not the same as the condition $\operatorname{Re}\left(\epsilon_{12}\right)=-\operatorname{Re}\left(\epsilon_{13}\right)$.)

\subsubsection{Discussion}

The exponential growth in the unstable modes for $w_{0}=3$ is consistent with the picture from Leonhardt et al. [61] that dynamical instabilities lead to a non-degenerate parametric amplification process. However, for a larger winding number of $w_{0}=10$ we have observed significant growth in modes $i=5,6$ for both stable and unstable configurations. Moreover, there is anomalous growth for mode $i=13$, that should be stable in both configurations according to the linear stability analysis.

The explanation for these qualitatively different regimes of behaviour, for winding numbers $w_{0}=3$ and $w_{0}=10$, is most likely due to the difference in nonlinearities for both cases. For the stationary solutions for the QdLN, found in the previous chapter, only $w_{0}$ and $V_{0}$ are free parameters - the nonlinearity $C$ is fixed by the condition for transonic flow (for the renormalised wavefunction). In particular, for $w_{0}=3$ we have $C=3.55 \times 10^{2}$, whereas for $w_{0}=10$ we have $C=3.95 \times 10^{3}$. Therefore it seems reasonable that the for $w_{0}=3$ the dynamics might be dominated by linear processes (ie. quadratic bosonic operator terms in the Hamiltonian), while for $w_{0}=10$ nonlinear processes (ie. higher order terms in the Hamiltonian) become important even for short timescales, due to the larger value of $C$. Further investigation of the nonlinear regime is outside the scope of the current work, but may be addressed at a later time. 

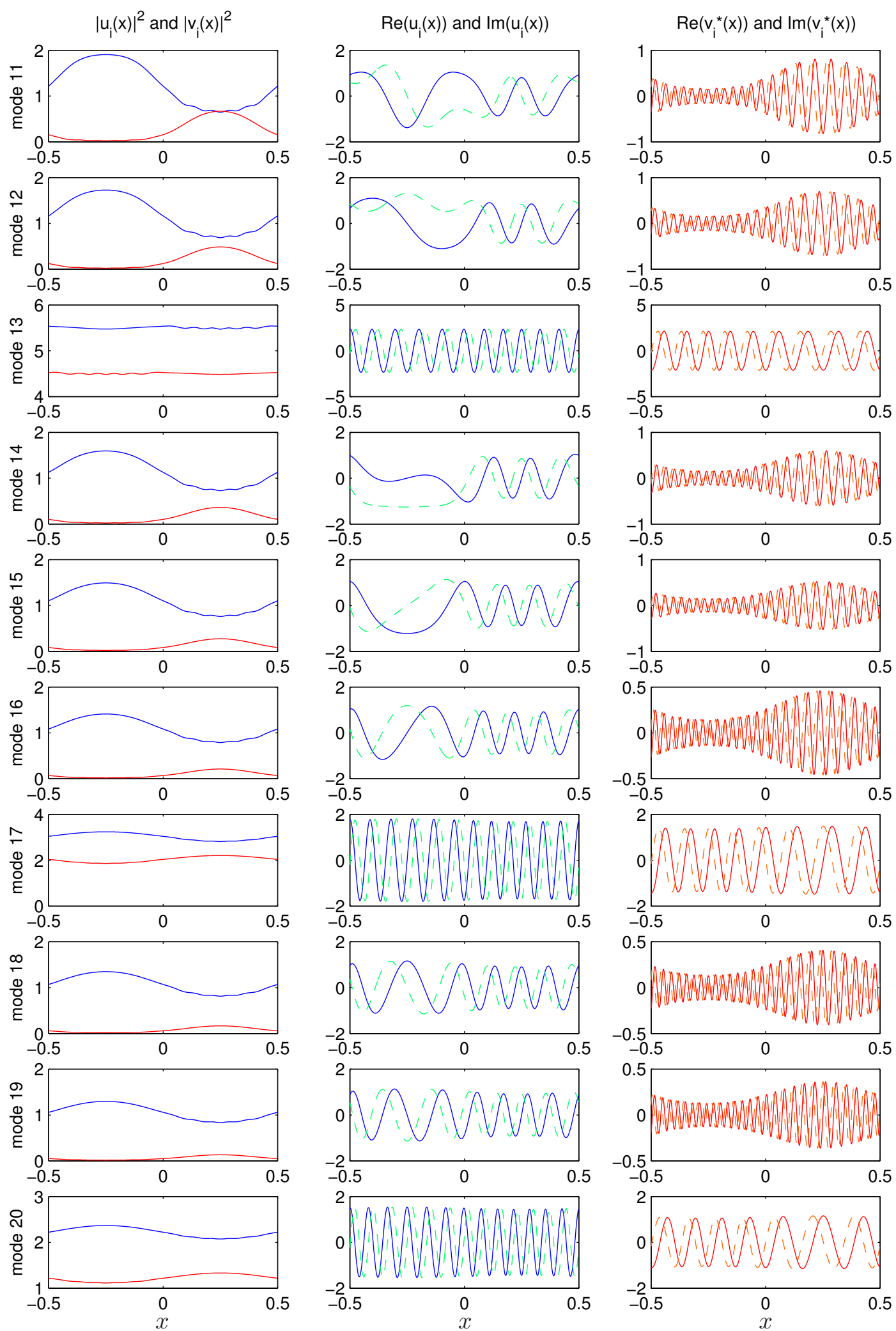

Figure 10.11: Dynamically unstable configuration: Mode function densities for $V_{0}=163.7$ and $w_{0}=3$. Modes $11 \leq i \leq 20$ are shown. In the first column $\left|u_{i}(x)\right|^{2}$ is given by the blue curve, and $\left|v_{i}(x)\right|^{2}$ is given by the red curve. In the second column $\operatorname{Re}\left(u_{i}(x)\right)$ is given by the solid blue curve, and $\operatorname{Im}\left(v_{i}(x)\right)$ is given by the dashed green curve. In the third column $\operatorname{Re}\left(v_{i}^{*}(x)\right)$ is given by the solid red curve, and $\operatorname{Im}\left(v_{i}^{*}(x)\right)$ is given by the dashed orange curve. 


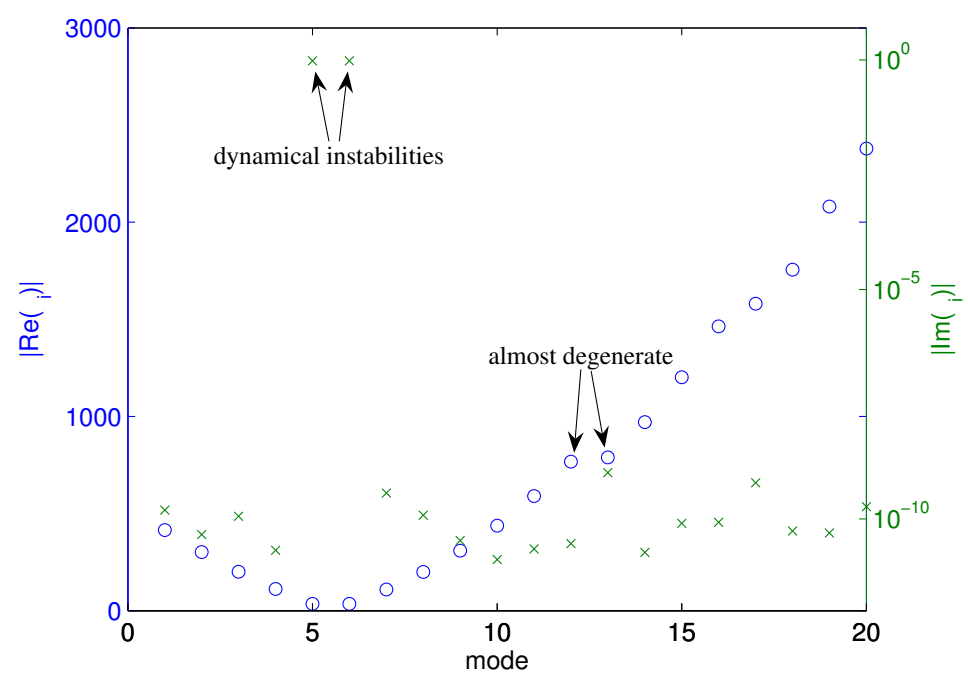

Figure 10.12: First 20 eigenvalues $\epsilon_{i}$ for configuration with $V_{0}=139.2$ and $w_{0}=10$. The blue circles (left axis) give $\left|\operatorname{Re}\left(\epsilon_{i}\right)\right|$ whereas the green crosses (right axis) give $\left|\operatorname{Im}\left(\epsilon_{i}\right)\right|$. Note that $\operatorname{Re}\left(\epsilon_{i}\right)<0$ for modes $i \leq 5$ and $\operatorname{Re}\left(\epsilon_{i}\right)>0$ for modes $i \geq 6$.

\subsection{Connection with Hawking effect}

\subsubsection{Hawking effect in the QdLN}

Despite previous studies, the question of whether the Hawking effect will occur for a black holewhite hole configuration in a BEC — such as the QdLN geometry — has not yet been satisfactorily resolved. Based on our results for the QdLN, we believe the closest analogy to the Hawking effect is given by the growth in dynamically unstable modes, as described by non-degenerate parametric amplification [61].

Our argument proceeds as follows. The dynamical instabilities can be described by the nondegenerate parametric amplification Hamiltonian (10.37). The symmetry of the BdG equations means that the dynamical instability must occur for a pair of eigenvalues such that $\epsilon_{i}=-\epsilon_{j}$. The physical role of the instability is thus to deplete the condensate, while creating correlated pairs of excitations with positive and negative energies. For the narrow regions of instability we have found, only two modes participate in this process. Therefore, while the statistics for nondegenerate parametric amplification may be thermal for the outgoing radiation [33], the spectrum is not Planckian as with the semiclassical result $[103,118]$. It should be emphasised that this picture is only valid at low winding numbers and for short times, when nonlinear processes do not dominate the dynamics.

This point of view is reinforced by the derivation of the Hawking effect as a tunneling process by Parikh and Wilczek [168]. This process occurs when pairs of positive and negative energy particles are created near the horizon, with either the positive energy particle tunneling out of the black hole horizon, or the negative energy particle tunneling into the black hole. The process is subject to energy conservation, which leads to corresponding decrease in the black hole size (and an increasing emission temperature). 
For the QdLN there are two necessary modifications to the semiclassical result for Hawking radiation, owing to the geometry and quantised nature of the system. These are:

Quantised circulation: The winding number $w_{0}$ must be conserved for the superfluid flow, and this inhibits certain transitions of the system. Since the stationary state is fully determined by the parameters $w_{0}$ and $V_{0}$, the system cannot relax to stationary state for a lower value of $w_{0}$ due to quasiparticle growth without a corresponding change in $V_{0}$.

Discrete spectrum: The spectrum for the trapped periodic system is discrete. The presence of dynamical instabilities depends on the values of $w_{0}$ and $V_{0}$. For a small value of $w_{0}$ there are only a few available negative eigenvalues, and so the opportunities for the quantum degeneracy which appear to be required for a dynamical instability are diminished. Thermodynamic instability (ie. negative eigenvalues) without quantum degeneracy does not lead to quasiparticle creation under Hamiltonian evolution, and therefore cannot be associated with the Hawking effect. The result is not so clear for large winding numbers, where nonlinear processes become important, and where there are a greater number of negative eigenvalues available that might lead to instabilities. It is worth noting that when the horizon area of a cosmological black hole is quantised, this similarly leads to a discrete spectrum for emission [243].

\subsubsection{Open issues}

To further justify our point of view, we must relate our work to the semiclassical prediction of the Hawking effect for analogue fluid models. In particular, there are a number of assumptions and approximations for the semiclassical treatment, which may not be valid for the QdLN, which is a quantum system. We note in particular the following outstanding issues:

\section{Approximate geometries}

As discussed in section 9.1, the investigations from Garay et al. $[149,161]$ and Leonhardt et al. [61,225] each used an approximate ansatz to describe a sonic horizon geometry. Therefore, the stability analysis for each geometry was not based on stationary solutions of the GPE, and so the results found there are necessarily approximate even within mean field theory (which itself is approximate).

\section{Hydrodynamics and geometric acoustics}

The original prediction of the Hawking effect in a fluid model $[103,118]$ depends on two assumptions. Firstly, the acoustic approximation (or hydrodynamic approximation) should be valid so that we only consider modes in a regime where the dispersion is linear. Secondly, that geometric acoustics apply — that is, a mode's wavelength is short compared to the scale over which the geometry changes.

Acoustic approximation: The well-known result from applying Bogoliubov theory to a weakly interacting Bose gas is that the excitation spectrum exhibits superluminal dispersion. Specifically, for the homogeneous system the low energy modes have linear dispersion (ie. phonon-like excitations), whereas the high energy modes have quadratic dispersion 
(ie. particle-like excitations). Standard derivations of the Hawking effect [162, 163, 170], as well as predictions for analogue fluid models $[103,118]$ assume a linear dispersion relation - ie. for an analogue model, that the acoustic approximation is valid. In fact, the effect of modified dispersion relations (MDRs) on the prediction of Hawking radiation has been investigated extensively [173-180]. The results from these studies indicate that the Hawking effect is relatively insensitive to the high frequency dispersion of the modes, and this resolves the so-called Trans-Planckian problem (see chapter 5). Specifically this result also applies to fluid models with acoustic horizons $[172,173]$.

Geometric acoustics: In the geometric acoustics limit, the propagation of short wavelength modes is determined by null geodesics of the effective spacetime - ie. in some sense, the mode behaves as a classical particle. This approximation is true when the mode's wavelength is short compared the scale over which the geometry changes. The significance of geometric acoustics is that the WKB (or semiclassical) approximation can then be used in the derivation of the Hawking effect (see $[61,103,168,170]$ for example).

In a classical fluid with linear dispersion, the assumption of geometric acoustics is reasonable since all modes are phononic, and this underlies the prediction of a Planckian spectrum for the analogue Hawking effect $[103,118]$. On the other hand, for a trapped BEC, the spectrum is discrete and exhibits superluminal dispersion, so that it may not be possible to find a regime where both the acoustic approximation and geometric acoustics are satisfied. Moreover, as we have seen, for the inhomogeneous system the mode structure is non-trivial, which may lead to some modifications to the predictions of the classical fluid theory.

\section{Black hole-white hole configuration}

Corley and Jacobson [244] have shown that black hole-white hole configurations lead to a black hole laser instability. In particular, if the dispersion is superluminal, there is exponential growth of Hawking radiation for a bosonic field due to repeated scattering between an outer and inner horizon. It should be emphasised that for this effect to occur the dispersion does not require complex frequency components. Barcelo et al. [245] have undertaken a numerical study of a black hole-white hole configuration and shown that when periodic boundary conditions are imposed, the instability may not occur in some cases. This result is consistent with the previously mentioned studies from Garay et al. [149,161] and Leonhardt et al. [61,225].

\section{Back-reaction}

The problem of back-reaction for an acoustic black hole has been previously addressed by Balbinot et al. [185]. There, by analysing a single de Laval nozzle geometry, it was shown that the emission temperature of an acoustic black hole decreases with time, in contrast with cosmological black holes where the temperature increases with time. This effect is associated with a slowing of the background flow.

For a BEC, the Bogoliubov theory gives the linear excitations of the system, and therefore necessarily neglects the back-reaction of quasiparticle modes on the condensate mode, as 
well as neglecting any higher order processes between the modes such as Beliaev damping [3]. Therefore, although the quasiparticles of the system may be described adequately by Bogoliubov theory, the long term dynamics might be dominated by nonlinear processes due to back-reaction. These effects are included in the effective Hamiltonian (1.1) for a weakly interacting BEC.

\subsubsection{Interpretation of Hawking effect for the QdLN}

Our investigation of the QdLN addresses all of the above issues on some level. The linear stability analysis and dynamics is based on the effective quantum field theory for the BEC, and therefore includes features neglected in the semiclassical treatment of analogue fluid models. In particular, we can address the issues from above:

\section{Approximate geometries}

Our analysis of the QdLN uses stationary solutions of the GPE, which are found numerically without any approximation.

\section{Hydrodynamics and geometric acoustics}

The stability analysis and dynamical simulations for the QdLN are both based on the GPE, which includes the quantum pressure term. Therefore the hydrodynamic approximation is not required or enforced. Moreover, the analysis includes modes of all wavelength scales (up to a cut-off) and so we do not make use of the geometric acoustics approximation.

However, it is worth considering in what regime the semiclassical derivation of the Hawking effect might be applicable for the QdLN. In this case the description of the BEC is given by classical fluid dynamics.

Recovering the hydrodynamic regime: When $C, V_{0} \gg 1$, we can see from the GPE (9.19) that the quantum pressure term can be neglected and the system is adequately described by hydrodynamics. In fact, as the nonlinearity increases with winding number $w_{0}$, this means we require a large value of $w_{0}$. However, for the periodic system, the quantisation of circulation cannot be relaxed.

Geometric acoustics: For geometric acoustics to be valid, the wavelength of the mode should be less than the scale over which the geometry changes - that is, for the QdLN we require $\lambda \leq L$. We can estimate the required system parameters from the semiclassical result for the Hawking temperature. In particular the peak wavelength $\lambda_{\text {peak }}$ for the Hawking spectrum is given by (9.18). This requirement that $\lambda_{\text {peak }} / L \leq 1$ leads to the inequality

$$
1 \leq 2 \pi\left(\frac{j U_{1 \mathrm{D}}}{m}\right)^{1 / 3}\left(\frac{8 m}{3 V_{0}}\right)^{1 / 2}
$$

That is, the geometric acoustics approximation is valid for a large current $j$ and nonlinearity $U_{1 \mathrm{D}}$ (ie. a large winding number) and/or a small value for the potential depth $V_{0}$.

\section{Black hole-white hole configuration}


The QdLN corresponds to a black hole-white hole configuration. In this case two de Laval nozzles are required for hydrodynamic stability, the flow becoming supersonic at the first nozzle and subsonic at the second. Moreover, the periodicity of the system enforces a fixed winding number $w_{0}$ for the flow circulation. The resulting stability analysis indicates regions of instability depending on the values of $w_{0}$ and $V_{0}$. This is consistent with previous studies of acoustic black holes in periodic systems [149, 245]. It does not seem that the black hole laser instability studied by Corley and Jacobson [244] is relevant to the QdLN, because in that study periodic boundary conditions were not used.

\section{Back-reaction}

We have performed classical field simulations for the QdLN; this requires the propagating the multi-mode classical field according to the projected GPE (7.5). The dynamics therefore includes nonlinear interactions between the quasiparticle modes, which are necessarily neglected in the linear stability analysis. Therefore our method includes the effects of back-reaction since the quasiparticle modes interact with each other - as well as with the condensate mode - via the nonlinear interaction term. The constraint of phase quantisation (ie. a fixed winding number) means that the system cannot relax to quasi-stationary state that differs from the initial one. This observation is verified by the long time simulations for the unstable configuration with $w_{0}=3$.

\subsubsection{Limitations of classical field method}

It should be emphasised that there are some limitations of the classical field method that we have used to investigate the QdLN, and that our analysis may therefore not provide the full picture. In particular, when the nonlinearity is large (ie. for large winding number solutions of the QdLN) we expect the Bogoliubov theory for quasiparticles to break down. This means that the quasiparticle vacuum, which is included in the initial state for the TWA simulations, may lead to thermalisation (ie. heating) of the system [34,37], and that the Bogoliubov quasiparticle picture will no longer be valid. Moreover, the Bogoliubov quasiparticle description is only valid near zero temperature, which restricts the applicability of our theory. An extension of the model to finite temperatures is possible by including damping and noise terms, which leads to the stochastic Gross-Pitaevskii equation [39,55-57]. A related issue, is that the TWA can only be used to describe short time dynamics for the system. This is the case because the third order terms in the equation of motion for the Wigner function, are neglected for the TWA. These third order terms are proportional to the nonlinearity (see equation 1.25) and so we expect the TWA to be valid for long time dynamics only when the nonlinearity becomes small. The validity of the TWA for long time dynamics been investigated by Sinatra et al. [37], which led to the condition $\left|T_{\text {class }}-T\right| \ll T$ where $T_{\text {class }}$ is the equilibrium temperature of the classical field. Finally, it is worth mentioning that when the nonlinearity is large, the effects of three-body recombination should be included in the TWA simulations. See Norrie et al. [223] where this has previously been done. 


\subsection{Conclusions}

\subsubsection{Summary}

In this part of the thesis, we have introduced and analysed the quantum de Laval nozzle (QdLN), a configuration for a Bose-Einstein condensate that exhibits both a black and white sonic horizon. We summarise our results as follows:

- We introduce the quasi-stationary configuration for the QdLN, which is based on a periodic potential in a ring geometry. Using hydrodynamic theory we find transonic solutions, which are then used to find transonic stationary solutions of the Gross-Pitaevskii equation.

- We have performed a stability analysis for the transonic solutions, using the Bogoliubov-de Gennes equations, from which we have found the discrete eigenvalue spectrum and quasiparticle modes for the system. Moreover, there are regions of instability for certain values of the geometry parameters: the winding number $w_{0}$, and the potential depth $V_{0}$.

- In particular, we have focused on two different regimes: (i) a low winding number $w_{0}=3$ with either $V_{0}=140$ for a dynamically stable configuration, or $V_{0}=163.7$ for a dynamically unstable configuration; (ii) a larger winding number $w_{0}=10$ with either $V_{0}=100$ for a dynamically stable configuration, or $V_{0}=139.2$ for a dynamically unstable configuration.

- We have investigated the dynamics of each of these configurations using the truncated Wigner approximation for the simulations. Specifically, we have calculated the quasiparticle mode populations as a function of simulation time. The single trajectory results for the $w_{0}=3$ case with $V_{0}=163.7$ (unstable) leads to exponential growth in the unstable modes, which can be interpreted as non-degenerate parametric amplification. In contrast for $V_{0}=140$ (stable) there is negligible growth in these modes. The results for the larger winding number $w_{0}=10$, does not lead to a similarly straightforward interpretation. For both $V_{0}=100$ (stable) and $V_{0}=139.2$ (unstable) there is appreciable growth in several modes. This is indicative of higher order processes, neglected in the Bogoliubov quasiparticle picture. This suggestion is reinforced by the observation that for the $w_{0}=10$ case the nonlinearity is $C=3.95 \times 10^{3}$, which is significantly larger than the $w_{0}=3$ case where the nonlinearity is $C=3.55 \times 10^{2}$.

- We have discussed the connection of our results with the usual derivation of the Hawking effect for analogue fluid models. Although the QdLN exhibits a rich mode structure in all regimes of interest, dynamical instabilities only appear for certain values of $w_{0}$ and $V_{0}$. We are able to construct normalisable Bogoliubov modes for the dynamical instabilities, which couple equal and opposite real frequencies. This can lead to exponential growth for those modes and this represents the closest analogy with the Hawking effect for our quantum system. Moreover, for a large winding number we observe: (i) the number of unstable regions increases (due to the higher number of negative energy modes), although they become narrower; and (ii) the nonlinearity increases so that the system approaches the hydrodynamic regime. The combination of these effects indicates it may be possible 
to (approximately) describe the system by a classical fluid in some regime, for which the prediction of a thermal spectrum from Unruh [103] and Visser [224] becomes relevant.

\subsubsection{Outlook}

The QdLN is a fascinating system from a theoretical point of view. Although we have considered some important aspects of the system, there are certainly opportunities for further inquiry. We therefore suggest several directions any future theoretical studies could take:

- The QdLN exhibits a rich spectrum and structure for the quasiparticle modes. Even at relatively modest winding numbers (ie. $w_{0}=10$ ) the dynamics appear highly nonlinear. From our preliminary investigation in this regime, it is not yet clear what underlying nonlinear processes contribute to the mode dynamics, which could be the subject of further investigation.

- To explore previously unaccessible regimes the numerical procedure used here could be improved by the use of larger numerical grids. In particular, it is of considerable interest to examine the QdLN with large winding numbers, which corresponds to the hydrodynamic regime. In this case the non-trivial phase variations and the large nonlinearities would require large spatial and temporal grids respectively.

- The quasi-one dimensional model presented here could be extended to two or even three dimensions. The additional degrees of freedom may allow some lattitude in the choice of transonic stationary solutions, not available in the one dimensional case. However, it is anticipated that the numerics will become difficult in this case because the total number of grid points will need be large to correctly resolve the Bogoliubov mode functions. (Recall that for the one dimensional case, finding the spectrum from the $\mathrm{BdG}$ equations requires the diagonalisation of a matrix with $2 n_{x} \times 2 n_{x}$ elements.) 


\section{Appendix A}

\section{Q-function for different states}

\section{A.1 Values of parameters in particular cases.}

To fit the kinds of distribution in Fig.3.1 we determine the parameters $v, \sigma$ and $c$ by fitting the moments $\langle a\rangle,\left\langle a a^{\dagger}\right\rangle$ and $\left\langle a a a^{\dagger} a^{\dagger}\right\rangle$.

\section{Parameters for a number state}

The exact values for the quantities (3.6-3.10) in the case of a number state $|m\rangle$ are

$$
\begin{aligned}
\langle a\rangle & =0 \\
\left\langle a a^{\dagger}\right\rangle & =m+1 \\
\langle a a\rangle & =0 \\
\left\langle a a a^{\dagger}\right\rangle & =0 \\
\left\langle a a a^{\dagger} a^{\dagger}\right\rangle & =(m+1)(m+2)
\end{aligned}
$$

leading to the choice

$$
\begin{aligned}
v^{4} & =m^{2}+\frac{3}{2} m+\frac{1}{2} \\
\sigma & =m+1-\sqrt{m^{2}+\frac{3}{2} m+\frac{1}{2}} \\
& \approx 1 / 4 \quad \text { as } m \rightarrow \infty \\
c & =0 .
\end{aligned}
$$

The two quantities (A.3,A.4) are given exactly by this choice. 


\section{Parameters for a superposition of two number states}

For an equal superposition of the kind (3.2) that

$$
\begin{aligned}
v^{4} & =n^{2}+\frac{n}{2}-\frac{1}{8} \\
\sigma & =n+\frac{1}{2}-\sqrt{n^{2}+\frac{n}{2}-\frac{1}{8}} \\
c & =\frac{\sqrt{n}}{2 v} .
\end{aligned}
$$

The exact computation of the quantities (3.6-3.10) yields in this case

$$
\begin{aligned}
\langle a\rangle & =\frac{1}{2} \sqrt{n} \\
\left\langle a a^{\dagger}\right\rangle & =n+\frac{1}{2} \\
\langle a a\rangle & =0 \\
\left\langle a a a^{\dagger}\right\rangle & =\frac{1}{2}(n+1) \sqrt{n} \\
\left\langle a a a^{\dagger} a^{\dagger}\right\rangle & =(n+1)^{2}
\end{aligned}
$$

The first, second and last of these are fitted exactly, leading to the approximate values

$$
\begin{aligned}
\langle a a\rangle & \approx\left(v^{2}+\sigma\right) c^{4} \\
& =\left(n+\frac{1}{2}\right) \frac{n^{2}}{16\left(n^{2}+n / 2-1 / 8\right.} \\
\left\langle a a a^{\dagger}\right\rangle & \approx\left(v^{3}+3 \sigma v\right) c \\
& =\frac{1}{2} \sqrt{n}\left(3\left(n+\frac{1}{2}\right)-2 \sqrt{n^{2}+n / 2-1 / 8}\right)
\end{aligned}
$$

Even for $n=1$ these are very tolerable approximations; (A.18) is diminished by comparison with $\left\langle a a^{\dagger}\right\rangle$ by a factor of $c^{4} \approx 0.045$, compared to the exact value of 0 . For large $n$ (A.19) becomes equal to the true value (A.16), and even for $n=1$ the true and the model results differ by less than $8 \%$.

\section{Parameters for a coherent state}

For a coherent state $|\beta\rangle$, where $\beta \equiv m$ is taken to be real, the quantities take the form

$$
\begin{aligned}
\langle a\rangle & =\beta \\
\left\langle a a^{\dagger}\right\rangle & =\beta^{2}+1 \\
\langle a a\rangle & =\beta^{2} \\
\left\langle a a a^{\dagger}\right\rangle & =\beta\left(\beta^{2}+2\right) \\
\left\langle a a a^{\dagger} a^{\dagger}\right\rangle & =\beta^{4}+4 \beta^{2}+2
\end{aligned}
$$


from which we can compute

$$
\begin{aligned}
v^{4} & =\beta^{4}+\beta^{2}+\frac{1}{2} \\
\sigma & =\beta^{2}=1-\sqrt{\beta^{4}+\beta^{2}+\frac{1}{2}} \\
c & =\frac{\beta}{v} \approx 1-\frac{1}{2 \beta^{2}} \quad \text { as } \beta \rightarrow \infty
\end{aligned}
$$

It is easy to check that for large $\beta$ the quantities (A.22,A.23) are correctly given, and they are of course correct for $\beta=0$. For $\beta=1$, we find that $\langle a a\rangle=0.8$ instead of the exact value of 1 , and $\left\langle a a a^{\dagger}\right\rangle=2.84$, instead of the exact value of 3 .

\section{A.2 A rigorous lower bound on the energy}

As a spinoff from our methodology of section 3.1, we find that we can also develop a rigorous lower bound for the energy as a function of the mean-field, and we can evaluate the predictions given by assuming the lower bound is equal to the ground-state energy.

To see this we note the mean energy, which is given by (3.25) in the mean-field approximation, is parameterised by number variance $\operatorname{var}[N]$ and the mean-field $\langle X\rangle$. Then we know that $\operatorname{var}[N]$ satisfies the two inequalities (3.16) and (3.20), which we can combine together as

$$
\operatorname{var}[N] \geq \max \left(\frac{\langle X\rangle^{2}}{4\left\langle Y^{2}\right\rangle}, \delta n(1-\delta n)\right) .
$$

We can develop a lower bound on $\left\langle Y^{2}\right\rangle$ by noting that

$$
\left\langle Y^{2}\right\rangle=n-\frac{1}{2}-\left\langle X^{2}\right\rangle \leq n-\frac{1}{2}-\langle X\rangle^{2}
$$

so that we can deduce from (A.28) that

$$
\operatorname{var}[N] \geq \max \left(\frac{\langle X\rangle^{2}}{4\left(n-\frac{1}{2}-\langle X\rangle^{2}\right)}, \delta n(1-\delta n)\right),
$$

and that the mean energy (3.25) satisfies the lower bound

$$
\langle E\rangle \geq-Z\langle X\rangle^{2}+u\left\{2+n^{2}+3 n+\max \left(\frac{\langle X\rangle^{2}}{4\left(n-\frac{1}{2}-\langle X\rangle^{2}\right)}, \delta n(1-\delta n)\right)\right\}
$$

This bound is parameterised by the mean-field $\langle X\rangle$ only, so the rigorous lower bound is given by the value, $X_{\min }(u, n)$, of $\langle X\rangle$ which gives the minimum value, $E_{\min }(u, n)$, the right hand side of (A.31), and subject to the restriction that $\langle X\rangle \leq \sqrt{n-1}$ (the equality holding for a coherent state). These are easy to evaluate, and resulting chemical potential and mean-field are plotted in Fig.A.1. The results are less accurate than the phase space method (see Fig.3.3), but are still surprisingly good. 

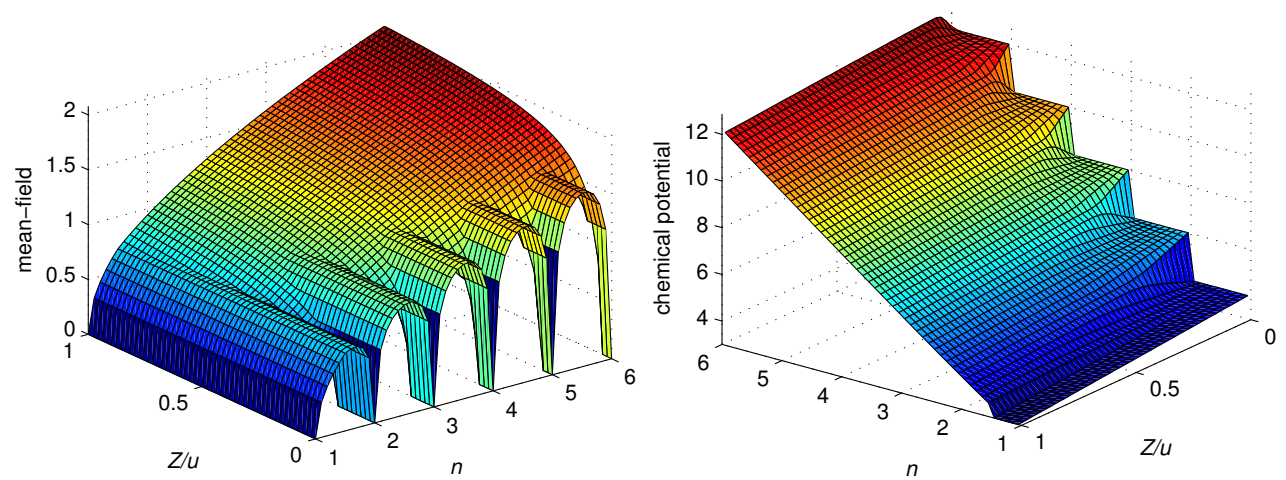

Figure A.1: Ground state phase diagram calculated using rigorous lower bound on energy (A.31) in Q-function representation 


\section{Appendix B}

\section{Numerical methods}

In this appendix we outline the numerical algorithm for integrating the projected Gross-Pitaevskii equation, which is used in parts II and III of this thesis, for simulating the dynamics of a BoseEinstein condensate via the truncated Wigner approximation. The algorithm is based on the Fourth-order Runge Kutta in the Interaction Picture (RK4IP) algorithm as developed previously by Caradoc-Davies [211]. The projector is incorporated into the algorithm following Norrie [210]. Note that in the following discussion, all parameters are rescaled to be dimensionless.

\section{B.1 Projected Gross-Pitaevskii equation}

The projected Gross-Pitaevskii equation (PGPE) given by (7.5) can be written in dimensionless form as:

$$
\begin{aligned}
i \frac{\partial \psi}{\partial t} & =\hat{D} \psi+\hat{P}\{\hat{N} \psi\} \\
\hat{D} & =-\frac{1}{2} \nabla^{2} \quad \text { (Dispersive term) } \\
\hat{N} & =V_{\text {ext }}+C|\psi|^{2} \quad \text { (Nonlinear term) }
\end{aligned}
$$

Expanding the field in some basis:

$$
\hat{\psi}(\mathbf{x}, t)=\sum_{j \in L} \phi_{j}(\mathbf{x}) \alpha_{j}(t)
$$

where $L$ represents a low-energy subspace of the system, the projector is given by its action on some function $f(\mathbf{x})$ as:

$$
\hat{P}\{f(\mathbf{x})\}=\sum_{j \in L} \phi_{j}(\mathbf{x}) \int d \mathbf{x}^{\prime} \phi_{j}^{*}\left(\mathbf{x}^{\prime}\right) f\left(\mathbf{x}^{\prime}\right)
$$




\section{B.2 Plane-wave basis}

It is useful to introduce the plane-wave basis with the field given by a discrete Fourier transform

$$
\psi(\mathbf{x}, t)=\frac{1}{\sqrt{V}} \sum_{\mathbf{k} \in L} e^{i \mathbf{k} \cdot \mathbf{x}} \alpha_{\mathbf{k}}(t)
$$

and the mode amplitude by an inverse (continuous) Fourier transform

$$
\alpha_{\mathbf{k}}(t)=\frac{1}{\sqrt{V}} \int d \mathbf{x} e^{-i \mathbf{k} \cdot \mathbf{x}} \psi(\mathbf{x}, t)
$$

The advantage of using the plane-wave basis is two-fold:

- There exist efficient algorithms for performing the discrete Fourier transforms, which result from the numerical representation of (B.6) and (B.7) - we discuss this further in section B.5 below. Moreover, when the numerical grid for coordinate space is sufficiently dense, it is appropriate to implement the projector by a cut-off $\mathbf{k}_{\text {cut }}$ in momentum space, as the single particle states match the eigenstates of the system for momenta $\mathbf{k} \sim \mathbf{k}_{\text {cut }}$.

- The action of the Laplacian operator is diagonal in momentum space. That is:

$$
\hat{D} \psi \equiv-\frac{1}{2} \nabla^{2} \psi=\frac{1}{\sqrt{V}} \sum_{\mathbf{k} \in L} \frac{1}{2} k^{2} e^{i \mathbf{k} \cdot \mathbf{x}} \alpha_{\mathbf{k}}
$$

In the plane-wave basis the PGPE transforms to the equation of motion for the mode amplitudes as

$$
\frac{\partial \alpha_{\mathbf{k}}}{\partial t}=-i\left[\frac{1}{2} k^{2} \alpha_{\mathbf{k}}+\frac{1}{\sqrt{V}} \int d \mathbf{x}\left\{e^{-i \mathbf{k} \cdot \mathbf{x}}\left(V_{\mathrm{ext}}+C|\psi|^{2}\right) \psi\right\}\right]
$$

The projector explicitly requires that $\mathrm{k} \in L$ here.

\section{B.3 Interaction picture}

The solution of (B.9) is facilitated by transforming to the interaction picture [28]. We can transform to the interaction picture by

$$
\alpha_{\mathbf{k}}^{\mathrm{IP}}(t)=e^{i\left(t-t^{\prime}\right) k^{2} / 2} \alpha_{\mathbf{k}}(t)
$$

The PGPE in momentum space in the interaction picture is then

$$
\frac{\partial \alpha_{\mathbf{k}}^{\mathrm{IP}}}{\partial t}=-i e^{i\left(t-t^{\prime}\right) k^{2} / 2} \frac{1}{\sqrt{V}} \int d \mathbf{x}\left\{e^{-i \mathbf{k} \cdot \mathbf{x}}\left(V_{\mathrm{ext}}+C|\psi|^{2}\right) \psi\right\}
$$

Therefore the free rotation of each mode has been removed by the transformation — this leads to increased stability for the numerical algorithm, which we now outline. 


\section{B.4 Projected RK4IP algorithm}

For an ordinary differential equation of the form:

$$
\frac{d \mathbf{y}}{d t}=\mathbf{f}(\mathbf{y}, t)
$$

the fourth-order Runge-Kutta formula is given by [246]

$$
\begin{aligned}
\mathbf{h}_{1} & =\Delta t \mathbf{f}\left(\mathbf{y}_{n}, t_{i}\right) \\
\mathbf{h}_{2} & =\Delta t \mathbf{f}\left(\mathbf{y}_{i}+\mathbf{h}_{1} / 2, t_{i}+\Delta t / 2\right) \\
\mathbf{h}_{3} & =\Delta t \mathbf{f}\left(\mathbf{y}_{i}+\mathbf{h}_{2} / 2, t_{i}+\Delta t / 2\right) \\
\mathbf{h}_{4} & =\Delta t \mathbf{f}\left(\mathbf{y}_{i}+\mathbf{h}_{3}, t_{i}+\Delta t\right) \\
\mathbf{y}_{i+1} & =\mathbf{y}_{i}+\left[\mathbf{h}_{1}+2\left(\mathbf{h}_{2}+\mathbf{h}_{3}\right)+\mathbf{h}_{4}\right] / 6+O\left(\Delta t^{5}\right)
\end{aligned}
$$

This gives the propagation of the field $\mathbf{f}(\mathbf{y}, t)$ from $t_{i}$ to $t_{i+1}$ where the time-step is given by $\Delta t=t_{i+1}-t_{i}$.

The solution of (B.11) using the above algorithm can be optimised by choosing the reference time as $t^{\prime}=t_{i}+\Delta t / 2$ so that $\mathbf{h}_{2}$ and $\mathbf{h}_{3}$ are evaluated at the origin of the interaction picture. This leads to the optimised algorithm:

$$
\begin{aligned}
\alpha_{\mathbf{k}}^{\mathrm{IP}}\left(t_{i}\right) & =e^{-i \Delta t k^{2} / 4} \alpha_{\mathbf{k}}\left(t_{i}\right) \\
h_{1} & =-i \Delta t e^{-i \Delta t k^{2} / 4} \frac{1}{\sqrt{V}} \int d \mathbf{x}\left\{e^{-i \mathbf{k} \cdot \mathbf{x}}\left(V_{\mathrm{ext}}\left(t_{i}\right)+C\left(t_{i}\right)\left|\psi_{1}\right|^{2}\right) \psi_{1}\right\} \\
h_{2} & =-i \Delta t \frac{1}{\sqrt{V}} \int d \mathbf{x}\left\{e^{-i \mathbf{k} \cdot \mathbf{x}}\left(V_{\mathrm{ext}}\left(t_{i}+\Delta t / 2\right)+C\left(t_{i}+\Delta t / 2\right)\left|\psi_{2}\right|^{2}\right) \psi_{2}\right\} \\
h_{3} & =-i \Delta t \frac{1}{\sqrt{V}} \int d \mathbf{x}\left\{e^{-i \mathbf{k} \cdot \mathbf{x}}\left(V_{\mathrm{ext}}\left(t_{i}+\Delta t / 2\right)+C\left(t_{i}+\Delta t / 2\right)\left|\psi_{3}\right|^{2}\right) \psi_{3}\right\} \\
h_{4}^{*} & =-i \Delta t \frac{1}{\sqrt{V}} \int d \mathbf{x}\left\{e^{-i \mathbf{k} \cdot \mathbf{x}}\left(V_{\mathrm{ext}}\left(t_{i}+\Delta t\right)+C\left(t_{i}+\Delta t\right)\left|\psi_{4}\right|^{2}\right) \psi_{4}\right\} \\
\alpha_{\mathbf{k}}\left(t_{i+1}\right) & =e^{-i \Delta t k^{2} / 4}\left(\alpha_{\mathbf{k}}^{\mathrm{IP}}\left(t_{i}\right)+\frac{1}{6}\left(h_{1}+2\left(h_{2}+h_{3}\right)\right)\right)+\frac{1}{6} h_{4}^{*}
\end{aligned}
$$

where we have defined the following:

$$
\begin{aligned}
& \psi_{1}=\frac{1}{\sqrt{V}} \sum_{\mathbf{k} \in L} e^{i \mathbf{k} \cdot \mathbf{x}} \alpha_{\mathbf{k}}\left(t_{i}\right) \\
& \psi_{2}=\frac{1}{\sqrt{V}} \sum_{\mathbf{k} \in L} e^{i \mathbf{k} \cdot \mathbf{x}}\left(\alpha_{\mathbf{k}}^{\mathrm{IP}}\left(t_{i}\right)+h_{1} / 2\right) \\
& \psi_{3}=\frac{1}{\sqrt{V}} \sum_{\mathbf{k} \in L} e^{i \mathbf{k} \cdot \mathbf{x}}\left(\alpha_{\mathbf{k}}^{\mathrm{IP}}\left(t_{i}\right)+h_{2} / 2\right) \\
& \psi_{2}=\frac{1}{\sqrt{V}} \sum_{\mathbf{k} \in L} e^{i \mathbf{k} \cdot \mathbf{x}} e^{-i \Delta t k^{2} / 4}\left(\alpha_{\mathbf{k}}^{\mathrm{IP}}\left(t_{i}\right)+h_{3}\right)
\end{aligned}
$$

Note that to provide the most general form of the algorithm, both $V_{\mathrm{ext}}$ and $C$ are written as time- 
dependent terms. However, for many situations of interest these terms are time-independent.

\section{B.5 Numerical grids}

The numerical implementation of the projected RK4IP algorithm requires a choice of discrete grid for the coordinate $(\mathbf{x})$ and momentum $(\mathbf{k})$ spaces. The practical implementation of the transforms between coordinate and momentum space uses Fast Fourier Transforms (FFTs) [247], which are most efficient for a uniform grid with $N=2^{n}$ points for some positive integer $n$. Moreover, FFTs are periodic so that the end points of the grid should not be repeated. If we consider a onedimensional system on a domain $[-L / 2, L / 2]$, we therefore have the following choice of grids:

1. Left aligned: $x_{j} \in[-L,-L+\Delta x, \ldots, L-2 \Delta x, L-\Delta x]$

2. Right aligned: $x_{j} \in[-L+\Delta x,-L+2 \Delta x, \ldots, L-\Delta x, L]$

3. Centre aligned: $x_{j} \in[-L+\Delta x / 2,-L+3 \Delta x / 2, \ldots, L-3 \Delta x / 2, L-\Delta x / 2]$

where $\Delta x=L / N$. We henceforth choose the left aligned grid for convenience. The corresponding momentum space grid is

$$
k_{j} \in \frac{2 \pi}{L}[0,1,2, \ldots, N / 2-2, N / 2-1,-N / 2,-N / 2+1, \ldots,-2,-1]
$$

The extension to two or three dimensions is straightforward, although it should be noted that the FFT algorithm requires the same number of points for each dimension with $N_{x}=N_{y}=N_{z}$. The fft and (inverse) ifft functions in Matlab are based on the FFTW library [247]. In this case, the numerical implementation of (B.6) and (B.7) is given respectively by

$$
\psi\left(x_{j}\right)=\frac{\sqrt{V}}{\Delta V} \text { ifft }\left[\alpha\left(k_{j}\right)\right]
$$

and

$$
\alpha\left(k_{j}\right)=\frac{\Delta V}{\sqrt{V}} \operatorname{fft}\left[\psi\left(x_{j}\right)\right]
$$

where $\Delta V$ is the volume element for the discretised grid and where $\psi\left(x_{j}\right)$ and $\alpha\left(k_{j}\right)$ are arrays.

\section{B.6 Numerical stability and accuracy}

In order to ensure that the projected RK4IP algorithm leads to numerically accurate solutions, there are several diagnostics that should be checked, as well as guidelines for choosing a suitable time step. 


\section{B.6.1 Checking stability}

\section{Convergence}

Numerical convergence can be checked by comparing the solution from a single time step to the case where the time step is halved and the field recomputed after two half steps. If the projected RK4IP converges these solutions should be the same to within numerical precision. By comparing whole and half time step solutions for a long simulation time (ie. consisting of many time steps $m$ and $2 m$ respectively) we can check the global convergence of the algorithm.

\section{Preserved quantities: norm and energy}

It is straightforward to show that the GPE is associated with conservation laws for the normalisation and energy of the field [3]. In particular, the GPE preserves the normalisation of the field $N=\int d \mathbf{x}|\psi|^{2}$ (this is consistent with the fact that the many-body Hamiltonian commutes with the number operator). Therefore the convergence of the algorithm can be ensured by choosing a time step small enough to give a constant normalisation. Moreover, for the time-independent Hamiltonian, the energy must be conserved, which serves as an another useful diagnostic check.

\section{B.6.2 Time step}

The time dynamics of the condensate mode is determined by a term of the form $\sim e^{-i \mu t}$ ( $\mu$ is the dimensionless chemical potential). Therefore to represent this phase factor accurately the Nyquist criterion means we require a time step:

$$
\Delta t \ll \frac{\pi}{\mu}
$$

A secondary requirement is given by noting that the shortest time scale for excitations is given by the evolution of the free particle modes. Therefore we have:

$$
\Delta t \ll \frac{2 \pi}{k^{2}}
$$

In general it is necessary to choose a time step that satisfies both (B.31) and (B.32). 


\section{Appendix C}

\section{Bogoliubov-de Gennes equations for PGPE}

We calculate the excitation spectrum (the dispersion curve) for a homogeneous condensate with steady flow, starting with the projected Gross-Pitaevskii equation (PGPE). The projected subspace in this case is given by the momentum grid ${ }^{1}$.

The projected GPE is given by

$$
i \hbar \frac{\partial \psi}{\partial t}=\left[-\frac{\hbar^{2}}{2 m} \nabla^{2}+V_{e x t}\right] \psi+\hat{P}\left\{U_{0}|\psi|^{2} \psi\right\}
$$

The wavefunction is given in an arbitrary basis set $\phi_{n}$ by

$$
\psi(\mathbf{x}, t)=\sum_{n} c_{n}(t) \phi_{n}(\mathbf{x})
$$

The action of the projector on some function $F(\mathbf{x})$ is given by

$$
\hat{P} F(\mathbf{x})=\sum_{k} \phi_{k}(\mathbf{x}) \int \phi_{k}^{*}\left(\mathbf{x}^{\prime}\right) F\left(\mathbf{x}^{\prime}\right) d \mathbf{x}^{\prime}
$$

where $\mathbf{k} \in G$ for a discrete momentum grid $G$. In the one dimensional case $-\bar{k} \leq k \leq+\bar{k}$ where $\bar{k}=\max (|k|)$. Following the usual linear response theory, we take

$$
\psi(\mathbf{x}, t)=e^{-i \mu t / \hbar}\left(\phi_{0}(\mathbf{x})+\delta \phi(\mathbf{x}, t)\right)
$$

where the ground state wavefunction $\phi_{0}$ satisfies the time-independent GPE. Linearizing (C.1) we get

$$
i \hbar \dot{\delta \phi}=\left[-\frac{\hbar^{2}}{2 m} \nabla^{2}+V_{e x t}-\mu\right] \delta \phi+U_{0} \hat{P}\left\{2\left|\phi_{0}\right|^{2} \delta \phi+\phi_{0}^{2} \delta \phi^{*}\right\}
$$

\footnotetext{
${ }^{1}$ This is not the same projector that is defined by the subspace orthogonal to the ground state wavefunction
} 


\section{Homogeneous condensate with steady flow}

Consider a one dimensional homogeneous system with $V_{\text {ext }}=0$ and periodic boundary conditions; the ground state wavefunction with momentum $q$ takes the form

$$
\phi_{0}(x)=\sqrt{n_{0}} e^{i q x}
$$

Equation (C.5) becomes

$$
i \hbar \dot{\delta \phi}=\left[-\frac{\hbar^{2}}{2 m} \nabla^{2}-\mu\right] \delta \phi+U_{0} \hat{P}\left\{2 n_{0} \delta \phi+n_{0} e^{i 2 q x} \delta \phi^{*}\right\}
$$

A component $k^{\prime}=k+q$ of $\delta \phi$ couples to a term $2 q-k^{\prime}=q-k$ via the final term. Therefore we can write

$$
\begin{aligned}
\delta \phi(x, t) & =\sum_{j}\left(u(x) e^{-i w_{j} t}+v^{*}(x) e^{i w_{j} t}\right) \\
& =\sum_{j, k}\left(c_{k+q} e^{-i\left(w_{j} t-(k+q) x\right)}+s_{q-k}^{*} e^{i\left(w_{j} t-(k-q) x\right.}\right)
\end{aligned}
$$

Substituting (C.8) into (C.5) and equating coefficients of $e^{-i \omega t}$ and $e^{i \omega t}$ respectively we get (dropping subscripts $j$ )

$$
\begin{aligned}
& \hbar \omega \sum_{k} c_{k+q} e^{i(k+q) x} \\
& =\left[L_{G P}+U_{0} n_{0}-\mu\right] \sum_{k} c_{k+q} e^{i(k+q) x}+U_{0} n_{0} \hat{P}\left\{\sum_{k} s_{q-k} e^{i(k+q) x}\right\} \\
& -\hbar \omega \sum_{k} s_{q-k} e^{i(k-q) x} \\
& \quad=\left[L_{G P}+U_{0} n_{0}-\mu\right] \sum_{k} s_{q-k} e^{i(k-q) x}+U_{0} n_{0} \hat{P}\left\{\sum_{k} c_{k+q} e^{i(k-q) x}\right\}
\end{aligned}
$$

with the GP operator for the homogeneous case

$$
L_{G P} \equiv-\frac{\hbar^{2}}{2 m} \nabla^{2}+U_{0} n_{0}
$$

Noting that $L_{G P} \phi_{0}=\mu \phi_{0}$ gives

$$
\mu=\frac{\hbar^{2} q^{2}}{2 m}+U_{0} n_{0}
$$

and only keeping components $e^{i p x}$ within the projected subspace can then write

$$
\hbar \omega c_{p+q}=\left(\frac{\hbar^{2}}{2 m}\left(p^{2}+2 p q\right)+U_{0} n_{0}\right) c_{p+q}+A_{p} U_{0} n_{0} s_{q-p}
$$




$$
-\hbar \omega s_{q-p}=\left(\frac{\hbar^{2}}{2 m}\left(p^{2}-2 p q\right)-U_{0} n_{0}\right) s_{q-p}+B_{p} U_{0} n_{0} c_{p+q}
$$

For convenience we have introduced

$$
\begin{aligned}
A_{p} & =1, & & p \leq \bar{k}-q \\
& =0, & & \text { otherwise } \\
B_{p} & =1, & & p \geq-\bar{k}+q \\
& =0, & & \text { otherwise }
\end{aligned}
$$

Equations (C.14) and (C.15) then lead to two different forms of the dispersion relation

Anomalous region: $p>\bar{k}-q$ or $p<-\bar{k}+q$

$$
\left(\hbar \omega-\frac{\hbar^{2} p q}{m}\right)^{2}=\left(\frac{\hbar^{2}}{2 m} p^{2}+U_{0} n_{0}\right)^{2}
$$

Normal region: $-\bar{k}+2 q \leq p \leq \bar{k}-2 q$

$$
\left(\hbar \omega-\frac{\hbar^{2} p q}{m}\right)^{2}=\frac{\hbar^{2}}{2 m} p^{2}\left(\frac{\hbar^{2}}{2 m} p^{2}+2 U_{0} n_{0}\right)
$$

This shows that the action of the projector means that the dispersion relations are discontinuous at $p=-\bar{k}+q$ and $p=\bar{k}-q$. Note that $q=0$ returns the usual form of the dispersion relation for a stationary homogeneous condensate. 


\section{List of Figures}

2.1 Treatment of hopping terms in the Bose-Hubbard model for a lattice of dimension $d=1$. The dashed arrows indicate those hopping terms $J a_{i} a_{j}^{\dagger}$ included by a mean-field approximation, whereas the solid arrows indicate those terms included explicitly in the Hamiltonian. We consider two formulations: (a) The hopping terms between the $i$ th site and its $z=2 d$ nearest neighbours are treated solely by a mean-field approximation; (b) The hopping terms between two adjacent sites $i$ and $i+1$ are included explicitly, whereas the hopping terms between each of these sites and and their (other) nearest $z^{\prime}=2 d-1$ neighbours are included using the mean-field approximation.

2.2 Ground state phase diagram for the Bose-Hubbard model using a self-consistent mean-field approach; the mean-field and chemical potential are both shown as a function of the relative interaction strength $Z / u$ and mean occupation number $n$ (normal ordered) . . . . . . . . . . . . . . . . . .

3.1 Q-function for the superposition of $n=6$ and $n=7$ states in proportion 1:0, $0.85: 0.15,0.5: 0.5,0.15: 0.85,0: 1 ;$ and for a coherent state with $\beta=\sqrt{6} . \ldots$

3.2 Level curves of energy (3.11) in the Q-function representation for three different cases are indicated by the dashed curves, with the energy decreasing for darker regions; the permissible parameter space for $v$ and $c$ is given by the bounds $v \geq 0$ and $0 \leq|c| \leq 1$ (for purely illustrative purposes we allow $c$ to be negative here because the problem is symmetrical about $c=0$ ) where $v$ is further bounded by (3.17). Since (3.11) has no local minima, the minimum energy occurs within the shown bounded region and subject to the constraint (3.22) derived from the phase-number uncertainty relation which is shown by the solid curve. The solution in each case is indicated by a circle. In the first case $(n=1, Z / u=0.5)$ this occurs for $c=0$ so that the mean-field is zero; in the second case $(n=1.5$, $Z / u=0.5)$ this occurs for a non-zero $c$ so that there is a mean-field. In both these cases, the solution saturates the upper bound for $v$. Conversely, in the third case ( $n=1.5, Z / u=10)$ where the lattice is weak, the solution occurs within the bounded region. . . . . . . . . . . . . . . . . .

3.3 Ground state results for Bose-Hubbard model with Q-function representation; the mean-field and chemical potential are shown as a function of relative interaction strength $Z / u$ and (anti-normal ordered) occupation $n \ldots \ldots \ldots$ 
3.4 Mean number occupation $n$ as a function of $\mu$ at $\nu=-500$ for the one site model. The Lagrange multiplier $\mu$ plays the role of (but is not equal to) the chemical potential; the horizontal regions are indicative of the system near the incompressible Mott insulator phase where $\partial \bar{n} / \partial \mu=0 \ldots \ldots \ldots \ldots$

3.5 Mean-field versus variance for commensurate (a) and incommensurate (b) mean site occupations for the one site case. The mean occupation number is indicated on the right axis of each plot. . . . . . . . . . . . . . .

4.1 Ground state phase diagram for two site Bose-Hubbard model using the Qfunction representation in the mean-field approximation. The mean-field and intersite correlations are shown as a function of the mean (anti-normal ordered) occupation $n$ and the relative interaction strength $z t / u \ldots \ldots \ldots$

4.2 A contour plot of the mean occupation $\bar{n}$ with respect to the Lagrange multipliers $\mu$ and $\nu$ reveals the Mott lobes for commensurate occupations, shown for $\bar{n}=1$, 2,3 , where the solutions to (4.65) are degenerate with respect to $\mu$, indicating the incompressibility of the phase. The Mott insulator phase does not exist for incommensurate occupations as shown by the remaining curves at $\bar{n}=0.5,1.5$, 2.5 and 3.5 from left to right in the figure. The dashed lines represent possible (non-degenerate) trajectories for the Mott insulator phase as discussed in section 4.2.1.

4.3 Mean-field as a function of number variance for commensurate (a) and incommensurate (b) occupations, both with $d=1$. In contrast to the one site approximation, in the commensurate case the Mott insulator phase with vanishing mean-field can occur for a non-zero number variance. . . . . . . . . . . . . .

4.4 Ground state results for the Bose-Hubbard model in the two site mean-field approximation. The mean-field and intersite correlations are shown as a function of the mean site occupation $\bar{n}$ and the relative interaction strength $z t / u$. A nonzero mean-field represents the superfluid regime. The mean-field is zero below a critical value $(z t / u)_{c}$ for commensurate occupations, indicating the onset of the Mott insulator phase. The intersite correlations are non-zero everywhere except for commensurate occupations in the limit of no tunneling where $z t / u=0$. The results are qualitatively similar for 1, 2, and 3 dimensional lattices. However, a feature of the $d=1$ phase diagram is the weak suppression of the mean-field at half-integer occupations, which is seen more seen more clearly in Fig.4.6. This may be an artifact of the two site approximation and requires further study. . . .

4.5 $d=1,2,3$ results: mean-field, intersite correlation and variance for commensurate occupations of $\bar{n}=1,2,3$. The dashed vertical lines indicate the corresponding positions (from left to right) of the phase boundary between the superfluid and Mott insulator phases as calculated using the perturbation theory outlined in section $4.2 .3 \ldots \ldots \ldots \ldots \ldots \ldots$ 
4.6 The mean-field (a) and intersite correlation (b) using a reduced basis of three states in the limit of zero tunneling $(z t / u=0)$ for $d=1,3$. The methodology is outlined in section 4.2.1. A feature of the $d=1$ case is the weak suppression of the mean-field around half-integer occupations. The $d=2$ results, which been omitted for clarity, are very close to the $d=3$ case. . . . . . . . . . . . . . .

5.1 Sonic horizons in a flowing fluid: the black and white hole horizons occur where the normal component of the fluid velocity is equal to the local speed of sound; between the two surfaces, a region called the ergoregion, the flow is supersonic. The behaviour of sound waves at different points in the fluid flow is shown by "sound cones" (the wavefronts of sound waves emitted from a point, defining the locus of null geodesics); the sound cones are tipped in the direction of flow in the supersonic region so that all null geodesics necessarily propagate in the direction of the flow. See the text for more details. . . . . . . . . . . . . .

6.1 Schematic of de Sitter expansion for the FRW analogue model; for region I $\left(t<t_{0}\right)$, there is no expansion and the mode solutions are those of a Minkowski spacetime with $U=U_{0}$; for region II $\left(t_{0}<t<t_{f}\right)$, there is a de Sitter type expansion and the mode solutions are non-trivial; finally for region III $\left(t>t_{f}\right)$ the expansion is turned off and the mode solutions are those of a Minkowski spacetime with $U=U_{0} / X \ldots \ldots \ldots \ldots \ldots \ldots \ldots$

6.2 Particle production for a de Sitter spacetime in the FRW analogue model: (a) $\bar{t}_{s}=1 \times 10^{-4}$ and a range of expansions $X$ as shown; (b) $X=2000$ and a range of expansion rates $\bar{t}_{s}$ as shown. The dimensionless scaling unit is $\bar{t}_{s}=t_{s} \hbar /\left(m L^{2}\right)$. In both cases the nonlinearity is $U_{0} N_{0}=10^{5} \hbar^{2} /\left(m L^{2}\right)$ (dimensionless units are introduced in chapter 7$) \ldots \ldots \ldots$. . . . . .

6.3 Equipartition of energy for a de Sitter spacetime in the FRW analogue model. In both cases the expansion is $X=1 \times 10^{5}$ and the nonlinearity is $U_{0} N_{0}=$ $10^{5} \hbar^{2} /\left(m L^{2}\right)$. The dimensionless energy is $\bar{\epsilon}_{k}=\epsilon_{k} m L^{2} / \hbar^{2}$ (dimensionless units are introduced in chapter 7$) \ldots \ldots \ldots \ldots$. . . . . . . . . .

6.4 Temperature from linear fit to equipartition for three different expansions $X$ for the FRW analogue model. The cosmological result from (6.93) is shown by the green solid curve. The dimensionless temperature is $\bar{T}=k_{B} T \hbar^{2} /\left(m L^{2}\right)$. The dimensionless scaling unit is $\bar{t}_{s}=t_{s} \hbar /\left(m L^{2}\right)$. The nonlinearity is $U_{0} N_{0}=$ $10^{5} \hbar^{2} /\left(m L^{2}\right)$ (dimensionless units are introduced in chapter 7 ). . . . . . . .

7.1 Number of atoms in the condensate vs time for different sets of parameters: each result is for a single trajectory with either a Bogoliubov (quasiparticle) initial vacuum or a white noise (momentum) vacuum. Other parameters are $N_{x}=N_{y}=128$ 
7.2 Equipartition of energy: linear fit of (7.30) to give temperature $\bar{T}$. The initial state includes vacuum fluctuations for a white noise vacuum (ie. 1/2 particle per momentum mode). Ergodicity has been assumed and the results are therefore time averaged over the final 50 simulation time points. Parameters are: (a) $C_{N L}=10^{4}, N_{0}=10^{5}$; (b) $C_{N L}=10^{5}, N_{0}=10^{7}$.

7.3 Calculated dispersion relation from (7.36). The numerical results are given by an density plot of the $\log$ of the power spectrum (renormalised so that the peak value is unity) with the darker regions indicating higher density. Results are ensemble averaged over 10 runs with parameters $N_{0}=10^{7}, N_{x}=N_{y}=128$. For comparison, also shown is the analytic result (white dashed curve) from the Bogoliubov theory (7.24), and the phonon branch of the spectrum $\omega \sim c k$ (blue solid curve) . . . . . . . . . . . . . . . . . .

8.1 de Sitter expansion: time dependence of Bogoliubov mode populations. Parameters are $C_{N L}(\bar{t}=0)=1 \times 10^{5}, N_{0}=10^{7}$ and $X=2 \times 10^{3}$. The blue points on each curve show where each mode crosses over from phonon to free-particle behaviour (as defined by $\bar{k}_{c}^{2} / 2=C_{N L}$ ). The green dashed curve shows the analytic prediction in the acoustic approximation from (6.68). The red solid curve shows the analytic prediction for a sudden transition from $(6.95) \ldots \ldots$. . . .

8.2 de Sitter model: density plot for renormalised wavefunction at beginning $(t=0)$ and at end $\left(t=t_{f}\right)$ of expansion. Parameters are $t_{s}=1 \times 10^{-5}, C_{N L}(t=0)=$ $1 \times 10^{5}$, and $N_{0}=10^{7} \ldots \ldots \ldots \ldots \ldots \ldots \ldots$

8.3 Modified de Sitter expansion: time dependence of Bogoliubov mode populations. Parameters are $C_{N L}(\bar{t}=0)=1 \times 10^{5}, N_{0}=10^{7}$ and $X=2 \times 10^{3}$. The lead in and lead out times are given by $t_{I}=t_{I I I}=\frac{1}{2} t_{s}$. The blue points on each curve show where each mode crosses over from phonon to free-particle behaviour (as defined by $\bar{k}_{c}^{2} / 2=C_{N L}$ ). The red solid curve shows the analytic prediction for a sudden transition from $(6.95) \ldots \ldots \ldots$. . . . . . . .

8.4 tanh expansion: time dependence of Bogoliubov mode populations. Parameters are $C_{N L}(\bar{t}=0)=1 \times 10^{5}, N_{0}=10^{7}$ and $X=10^{3}$. The blue points on each curve show where each mode crosses over from phonon to free-particle behaviour (as defined by $\bar{k}_{c}^{2} / 2=C_{N L}$ ). The green dashed curve shows the analytic prediction in the acoustic approximation from (6.79). The red solid curve shows the analytic prediction for a sudden transition from (6.95). . . . . .

8.5 Cyclic universe: Nonlinearity vs time (left) and time dependence of Bogoliubov mode populations (right) for a single cycle $(m=1)$ and for two different periods $t_{s}$. Parameters are $C_{N L}(\bar{t}=0)=1 \times 10^{5}, N_{0}=10^{7}$ for both cases. The red solid line shows the position of the peak wave-vector for parametric resonance from the analytic prediction (6.98). 
8.6 Cyclic universe: Nonlinearity vs time (left) and time dependence of Bogoliubov mode populations (right) for three different scenarios. Parameters are $C_{N L}(\bar{t}=$ $0)=1 \times 10^{5}, N_{0}=10^{7}$ for all cases. The red solid line shows the position of the peak wave-vector for parametric resonance from the analytic prediction (6.98) . . . . . . . . . . . . . . . . . . . . . . . .

8.7 Cyclic model: Density plot for renormalised wavefunction at beginning $(t=0)$ and at end $\left(t=t_{f}\right)$ of expansion. Parameters are $m=10, X=2, t_{s}=1 \times 10^{-4}$, $C_{N L}(t=0)=1 \times 10^{5}$, and $N_{0}=10^{7}$.

8.8 Cyclic model: Density plot for renormalised wavefunction at beginning $(t=0)$ and at end $\left(t=t_{f}\right)$ of expansion. Parameters are $m=10, X=2, t_{s}=2 \times 10^{-4}$, $C_{N L}(t=0)=1 \times 10^{5}$, and $N_{0}=10^{7}$.

8.9 Equipartition of energy at the final time for de Sitter expansion with four different expansion rates. Parameters are $C_{N L}(t=0)=1 \times 10^{5}, N_{0}=10^{7}$ and $X=2 \times 10^{3}$.

9.1 The chemical potential as a function of flow velocity at the waist $(x=0)$ as given by (9.34); when $\mu>\mu_{\text {crit }}$ there are two real positive solutions. The dashed line indicates the transonic condition where $c=v$ and a crossover is possible from the subsonic to supersonic regimes (or vice versa). Dimensionless parameters are $C=500, V_{0}=100$ and $w_{0}=10$ (the current is determined implicitly by this choice of parameters). . . . . . . . . . . . . . . . . .

9.2 velocity $(v)$ and speed of sound $(c)$ for two real and positive solutions to cubic given by (9.41) and (9.42); in both cases parameters are $C=500, V_{0}=100$ and $\mu=\mu_{\text {crit }}=5.99 \times 10^{3}$ for the case when $J=J\left(w_{0}=10\right)$. The third solution of the cubic is negative, giving a negative density, and is therefore omitted. When $J>J\left(w_{0}=10\right)$ the solutions become complex indicating an unstable flow. Note that for the critical case (b) the current corresponds to a winding number of $w_{0}=10$, whereas for the subcritical case (a) phase quantisation is violated. .

9.3 The potential (9.13) with $V_{0}=100$ (in dimensionless units, which have been introduced in section 9.4.4) . . . . . . . . . . . . . . . . . . .

9.4 Stationary state for $w_{0}=3$ and $V_{0}=100$; corresponds to $\mu=5.93 \times 10^{2}$ and

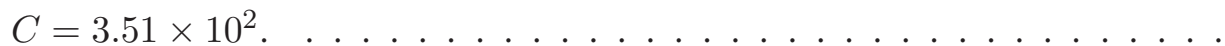

9.5 Stationary state for $w_{0}=10$ and $V_{0}=100$; corresponds to $\mu=5.99 \times 10^{3}$ and $C=3.95 \times 10^{3}$.

9.6 Stationary state for $w_{0}=50$ and $V_{0}=100$; corresponds to $\mu=1.48 \times 10^{5}$ and $C=9.87 \times 10^{4}$.

9.7 Nonlinearity $C$ as function of winding number $w_{0}$ for stationary solutions of QdLN with $V_{0}=100$. Note that the nonlinearity depends only very weakly on $V_{0}$, and reproducing this plot for any $V_{0} \leq 2000$ gives a quantitatively similar result. 
9.8 Estimated Hawking temperature as function of winding number $w_{0}$ for range of potential depths $V_{0}$. The data points have been calculated from $((9.61))$ by a numerical estimate of the surface gravity $\bar{g}_{H}$. The solid curves are given by the estimate from (9.16). . . . . . . . . . . . . . . . . .

10.1 Stability diagram of quantum de Laval nozzle: Largest imaginary component of eigenvalue for the Bogoliubov modes for a range of winding numbers as a function of $V_{0}$. The values of the imaginary eigenvalue component and $V_{0}$ are both given in the dimensionless units introduced in section 9.4.4. Each solution was found using a spatial grid of 256 points. . . . . . . . . . . . . . .

10.2 Eigenvalue spectrum (all negative eigenvalues and same number of lowest positive eigenvalues) of QdLN found from solutions of BdG equations. The red lines show the moduli of the negative eigenvalues and the blue lines show the positive eigenvalues. Eigenvalues have an imaginary component as indicated, only for a pair of eigenvalues that satisfies $\epsilon_{i}=-\epsilon_{j}$ exactly. Solutions have been found using a spatial grid of 1024 points. . . . . . . . . . . . . . . . . .

10.3 Dynamically unstable configuration: Orthogonal mode functions for $V_{0}=163.7$ and $w_{0}=3$. Modes $1 \leq i \leq 10$ are shown. In the first column $\left|u_{i}(x)\right|^{2}$ is given by the blue curve, and $\left|v_{i}(x)\right|^{2}$ is given by the red curve. In the second column $\operatorname{Re}\left(u_{i}(x)\right)$ is given by the solid blue curve, and $\operatorname{Im}\left(v_{i}(x)\right)$ is given by the dashed green curve. In the third column $\operatorname{Re}\left(v_{i}^{*}(x)\right)$ is given by the solid red curve, and $\operatorname{Im}\left(v_{i}^{*}(x)\right)$ is given by the dashed orange curve. Solutions have been found using a spatial grid of 1024 points.

10.4 Dynamically unstable configuration: Orthogonal mode functions for $V_{0}=139.2$ and $w_{0}=10$. Modes $1 \leq i \leq 10$ are shown. In the first column $\left|u_{i}(x)\right|^{2}$ is given by the blue curve, and $\left|v_{i}(x)\right|^{2}$ is given by the red curve. In the second column $\operatorname{Re}\left(u_{i}(x)\right)$ is given by the solid blue curve, and $\operatorname{Im}\left(v_{i}(x)\right)$ is given by the dashed green curve. In the third column $\operatorname{Re}\left(v_{i}^{*}(x)\right)$ is given by the solid red curve, and $\operatorname{Im}\left(v_{i}^{*}(x)\right)$ is given by the dashed orange curve. Solutions have been found using a spatial grid of 1024 points. . . . . . . . . . . . . . . . . . .

10.5 Bogoliubov mode populations for single trajectory from TWA evolution for parameters $V_{0}=140, w_{0}=3$, corresponding to a dynamically stable configuration. The simulation end time is $t=1 \ldots \ldots \ldots \ldots \ldots$

10.6 Bogoliubov mode populations for single trajectory from TWA evolution for parameters $V_{0}=163.7, w_{0}=3$, corresponding to a dynamically unstable configuration. The simulation end time is $t=1$. The dynamically unstable modes $(i=1,6)$ are indicated by the dark red curves. . . . . . . . . . . .

10.7 Bogoliubov mode populations for single trajectory from TWA evolution for parameters $V_{0}=163.7, w_{0}=3$, corresponding to a dynamically unstable configuration. The simulation end time is $t=5$. The dynamically unstable modes $(i=1,6)$ are indicated by the dark red curves. . . . . . . . . . . 
10.8 Coordinate space density vs time for unstable configuration with $w_{0}=3$ and $V_{0}=163.7$ : (a) gives the normalised density of the field by $n(x, t)=$ $|\psi(x, t)|^{2} / N_{0}$; (b) gives a intensity plot of the change in density from the initial state by $\Delta n(x, t)=n(x, t)-n(x, 0)$. Note that in plot (a) the high frequency noise has been filtered out during post-processing of the data. . . . . . . . . 170

10.9 Bogoliubov mode populations from TWA evolution for parameters $V_{0}=100$, $w_{0}=10$, corresponding to a dynamically stable configuration. The simulation end time is $t=0.1$. The quasiparticle number has been calculated by averaging over 40 trajectories. . . . . . . . . . . . . . . . .

10.10 Bogoliubov mode populations from TWA evolution for parameters $V_{0}=139.2$, $w_{0}=10$, corresponding to a dynamically unstable configuration. The simulation end time is $t=0.1$. The quasiparticle number has been calculated by averaging over 40 trajectories. The dynamically unstable modes $(i=5,6)$ are indicated by the dark red curves. . . . . . . . . . . . . . . . . .

10.11 Dynamically unstable configuration: Mode function densities for $V_{0}=163.7$ and $w_{0}=3$. Modes $11 \leq i \leq 20$ are shown. In the first column $\left|u_{i}(x)\right|^{2}$ is given by the blue curve, and $\left|v_{i}(x)\right|^{2}$ is given by the red curve. In the second column $\operatorname{Re}\left(u_{i}(x)\right)$ is given by the solid blue curve, and $\operatorname{Im}\left(v_{i}(x)\right)$ is given by the dashed green curve. In the third column $\operatorname{Re}\left(v_{i}^{*}(x)\right)$ is given by the solid red curve, and $\operatorname{Im}\left(v_{i}^{*}(x)\right)$ is given by the dashed orange curve. . . . . . . . . .

10.12 First 20 eigenvalues $\epsilon_{i}$ for configuration with $V_{0}=139.2$ and $w_{0}=10$. The blue circles (left axis) give $\left|\operatorname{Re}\left(\epsilon_{i}\right)\right|$ whereas the green crosses (right axis) give $\left|\operatorname{Im}\left(\epsilon_{i}\right)\right|$. Note that $\operatorname{Re}\left(\epsilon_{i}\right)<0$ for modes $i \leq 5$ and $\operatorname{Re}\left(\epsilon_{i}\right)>0$ for modes $i \geq 6$.

A.1 Ground state phase diagram calculated using rigorous lower bound on energy (A.31) in Q-function representation . . . . . . . . . . . . . . . . . . . 184 



\section{References}

[1] F. Dalfovo, S. Giorgini, L. P. Pitaevskii, and S. Stringari. Theory of Bose-Einstein condensation in trapped gases. Rev. Mod. Phys., 71:463, 1999.

[2] A. J. Leggett. Bose-Einstein condensation in the alkali gases: Some fundamental concepts. Rev. Mod. Phys., 73:307, 1999.

[3] L. Pitaevskii and S. Stringari. Bose-Einstein condensation. Oxford University Press, Oxford, first edition, 2002.

[4] C. J. Pethick and H. Smith. Bose-Einstein Condensation in Dilute Gases. Cambridge University Press, Cambridge, United Kingdom, first edition, 2001.

[5] A. Einstein. Sitzber. Kgl. Preuss. Akad. Wiss., page 3, 1925.

[6] S. N. Bose. Z. Phys., 26:178, 1924.

[7] F. London. The $\lambda$-phenomenon of liquid helium and the Bose-Einstein degeneracy. Nature, 141:643, 1938.

[8] N. N. Bogoliubov. J. Phys. (USSR), 11:23, 1947.

[9] O. Penrose and L. Onsager. Bose-Einstein Condensation and Liquid Helium. Phys. Rev., 104:576, 1956.

[10] E. P. Gross. Nuovo Cimento, 20:454, 1961.

[11] L. P. Pitaevskii. Zh. Eksp. Teor. Fiz., 40:646, 1961.

[12] M. H. Anderson, J. R. Ensher, M. R. Matthews, C. E. Wieman, and E. A. Cornell. Observation of Bose-Einstein Condensation in a Dilute Atomic Vapor. Science, 269:198, 1995.

[13] C. C. Bradley, C. A. Sackett, J. J. Tollett, and R. G. Hulet. Evidence of Bose-Einstein Condensation in an Atomic Gas with Attractive Interactions. Phys. Rev. Lett., 75(9):16871690, Aug 1995.

[14] K. B. Davis, M. O. Mewes, M. R. Andrews N. J. van Druten, D. S. Durfee, D. M. Kurn, and W. Ketterle. Bose-Einstein Condensation in a Gas of Sodium Atoms. Phys. Rev. Lett., 75(22):3969-3973, Nov 1995. 
[15] D. G. Fried, T. C. Killian, L. Willmann, D. Landhuis, S. C. Moss, D. Kleppner, and T. J. Greytak. Bose-Einstein Condensation of Atomic Hydrogen. Phys. Rev. Lett., 81(18):38113814, Nov 1998.

[16] S. L. Cornish, N. R. Claussen, J. L. Roberts, E. A. Cornell, and C. E. Wieman. Stable ${ }^{85} R b$ Bose-Einstein Condensates with Widely Tunable Interactions. Phys. Rev. Lett., 85(9):17951798, Aug 2000.

[17] F. Pereira Dos Santos, J. Léonard, J. Wang, C. J. Barrelet, F. Perales, E. Rasel, C. S. Unnikrishnan, M. Leduc, and C. Cohen-Tannoudji. Bose-Einstein Condensation of Metastable Helium. Phys. Rev. Lett., 86(16):3459-3462, Apr 2001.

[18] A. Robert, O. Sirjean, A. Browaeys, J. Poupard, S. Nowak, D. Boiron, C.I. Westbrook, and A. Aspect. A Bose-Einstein Condensate of Metastable Atoms. Science, 292(5516):461464, 2001.

[19] G. Modugno, G. Ferrari, G. Roati, R. J. Brecha, A. Simoni, and M. Inguscio. Bose-Einstein Condensation of Potassium Atoms by Sympathetic Cooling. Science, 294(5545):13201322, 2001.

[20] T. Weber, J. Herbig, M. Mark, H.-C. Nagerl, and R. Grimm. Bose-Einstein Condensation of Cesium. Science, 299(5604):232-235, 2003.

[21] I. Bloch. Exploring quantum matter with ultracold atoms in optical lattices. J. Phys. B: At. Mol. Opt. Phys.", 38(9):S629-S643, 2005.

[22] Q. Chen, J. Stajic, and K. Levin. Applying BCS-BEC Crossover Theory To High Temperature Superconductors and Ultracold Atomic Fermi Gases. Low Temperature Physics, 32:406, 2006.

[23] A.L. Fetter and A.A. Svidzinsky. Vortices in a trapped dilute Bose-Einstein condensate. Journal of Physics: Condensed Matter, 13(12):R135-R194, 2001.

[24] R. J. Ballagh and C. M. Savage. The Theory of Atom Lasers. Modern Physics Letters B, 14:153-188, 2000.

[25] W. P. Reinhardt and C.W. Clark. Soliton dynamics in the collisions of Bose-Einstein condensates: an analogue of the Josephson effect. J. Phys. B: At. Mol. Opt. Phys.", 30(22):L785-L789, 1997.

[26] T. Kinoshita, T. Wenger, and D. S. Weiss. Observation of a One-Dimensional TonksGirardeau Gas. Science, 305(5687):1125-1128, 2004.

[27] C. Barcelo and S. Liberati and M. Visser. Analogue Gravity. Living Rev. Rel., 8, 2006.

[28] A.L. Fetter and J.D. Walecka. Quantum Theory of Many-Particle Systems. Dover Publications Inc., 1971, 1999, 2003. 
[29] A. L. Fetter. Nonuniform states of an imperfect bose gas. Annals of Physics, 70:67-101, March 1972.

[30] C. W. Gardiner and P. Zoller. Quantum Noise. Springer-Verlag, Berlin, 3rd edition, 2004.

[31] C. W. Gardiner. Particle-number-conserving Bogoliubov method which demonstrates the validity of the time-dependent Gross-Pitaevskii equation for a highly condensed Bose gas. Phys. Rev. A, 56:1414, 1997.

[32] Y. Castin and R. Dum. Low-temperature Bose-Einstein condensates in time-dependent traps: Beyond the $U(1)$ symmetry-breaking approach. Phys. Rev. A, 57(4):3008-3021, Apr 1998.

[33] D. F. Walls and G. J. Milburn. Quantum Optics. Springer-Verlag, Berlin Heidelberg, first edition, 1994.

[34] M.J. Steel, M.K. Olsen, L.I. Plimak, P.D. Drummond, S.M. Tan, M.J. Collett, D.F. Walls, and R. Graham. Dynamical quantum noise in trapped Bose-Einstein condensates. Phys. Rev. A, 58:4824, 1998.

[35] A. Sinatra and Y. Castin and C. Lobo. A Monte Carlo formulation of the Bogolubov theory. Journal of Modern Optics, 47(14-15):2629, 2000.

[36] A. Sinatra, C. Lobo, and Y. Castin. Classical-Field Method for Time Dependent BoseEinstein Condensed Gases. Phys. Rev. Lett., 87(21):210404, Nov 2001.

[37] A. Sinatra, C. Lobo, and Y. Castin. The truncated Wigner method for Bose-condensed gases: limits of validity and applications. J. Phys. B: At. Mol. Opt. Phys., 35(17):35993631, 2002.

[38] C. W. Gardiner, J. R. Anglin, and T. I. A. Fudge. The Stochastic Gross-Pitaevskii equation. J. Phys. B: At. Mol. Opt. Phys., 35:1555, 2002.

[39] C. W. Gardiner and M. J. Davis. The Stochastic Gross-Pitaevskii equation:II. J. Phys. B: At. Mol. Opt. Phys., 36:4731, 2003.

[40] A. Polkovnikov. Quantum corrections to the dynamics of interacting bosons: Beyond the truncated Wigner approximation. Phys. Rev. A, 68:053604, 2003.

[41] A. A. Norrie, R. J. Ballagh, and C. W. Gardiner. Quantum turbulence and correlations in Bose-Einstein condensate collisions. Phys. Rev. A, 73(4):043617, 2006.

[42] A. A. Norrie, R. J. Ballagh, and C. W. Gardiner. Quantum Turbulence in Condensate Collisions: An Application of the Classical Field Method. Phys. Rev. Lett., 94(4):040401, 2005.

[43] P. D. Drummond and J. F. Corney. Quantum dynamics of evaporatively cooled BoseEinstein condensates. Phys. Rev. A, 60(4):R2661-R2664, Oct 1999. 
[44] P. Deuar and P. D. Drummond. Gauge $P$ representations for quantum-dynamical problems: Removal of boundary terms. Phys. Rev. A, 66(3):033812, Sep 2002.

[45] P. Deuar and P. D. Drummond. First-principles quantum dynamics in interacting Bose gases: I. The positive P representation. J. Phys. A: Math. Gen., 39(5):1163-1181, 2006.

[46] P. Deuar and P. D. Drummond. First-principles quantum dynamics in interacting Bose gases II: stochastic gauges. J. Phys. A: Math. Gen., 39(11):2723-2755, 2006.

[47] B. V. Svistunov. J. Mosc. Phys. Soc., 75:387, 1992.

[48] Yu. M. Kagan, B. V. Svistunov, and G. V. Shlyapnikov. Sov. Phys. JETP, 75:387, 1992.

[49] Yu. Kagan and B. V. Svistunov. Phys. Rev. Lett., 79:3331, 1997.

[50] R. J. Marshall, G. H. C. New, K. Burnett, and S. Choi. Exciting, cooling, and vortex trapping in a Bose-condensed gas. Phys. Rev. A, 59:2085, 1999.

[51] M. J. Davis, R. J. Ballagh, and K. Burnett. Dynamics of thermal Bose fields in the classical limit. J. Phys. B: At. Mol. Opt. Phys., 34:4487-4512, 2001.

[52] M. J. Davis, S. A. Morgan, and K. Burnett. Simulations of Bose Fields at Finite Temperature. Phys. Rev. Lett., 87:160402, 2001.

[53] M.J. Davis, S.A. Morgan, and K. Burnett. Simulations of thermal Bose fields in the classical limit. Phys. Rev. A, 65:053618, 2002.

[54] P. B. Blakie and M. J. Davis. Projected Gross-Pitaevskii equation for harmonically confined Bose gases at finite temperature. Phys. Rev. A, 72(6):063608, 2005.

[55] C. W. Gardiner, J. R. Anglin, and T. I. A. Fudge. The Stochastic Gross-Pitaevskii equation. J. Phys. B: At. Mol. Opt. Phys., 35:1555, 2002.

[56] A. S. Bradley and C. W. Gardiner. arXiv:cond-mat/0602162, 2006.

[57] A. S. Bradley, P. B. Blakie, and C. W. Gardiner. Properties of the stochastic Gross-Pitaevskii equation: finite temperature Ehrenfest relations and the optimal plane wave representation. J. Phys. B: At. Mol. Opt. Phys., 38:4259, 2005.

[58] M. J. Davis, C. W. Gardiner, and R. J. Ballagh. Quantum kinetic theory. VII. The influence of vapor dynamics on condensate growth. Phys. Rev. A, 62(6):063608, Nov 2000.

[59] H. T. C. Stoof. Coherent verses Incoherent Dynamics during Bose-Einstein Condensation in Atomic Gases. J. Low Temp. Phys., 114:11, 1999.

[60] C. W. Gardiner. Handbook of Stochastic Methods. Springer-Verlag, Berlin Heidelberg, third edition, 2004. 
[61] U. Leonhardt and T. Kiss and P. Ohberg. Theory of elementary excitations in unstable Bose-Einstein condensates and the instability of sonic horizons. Phys. Rev. A, 67:033602$1,2003$.

[62] P. Jain and C. W. Gardiner. A phase-space method for the Bose-Hubbard model: application to mean-field models. J. Phys. B: At. Mol. Opt. Phys., 37(18):3649-3680, 2004.

[63] D. Jaksch, C. Bruder, J. I. Cirac, C. W. Gardiner, and P. Zoller. Cold Bosonic Atoms in Optical Lattices. Phys. Rev. Lett., 81(15):3108, October 1998.

[64] M. Greiner, O. Mandel, T. Esslinger, T. W. Hänsch, and I. Bloch. Quantum phase transition from a superfluid to a Mott insulator in a gas of ultracold atoms. Nature, 415:39, 2002.

[65] D.S. Rokhsar and B.G. Kotliar. Gutzwiller projection for bosons. Phys. Rev. B., 44(18):10328, 1991.

[66] W. Zwerger. Mott-Hubbard transition of cold atoms in optical lattices. J. Opt. B: Quantum Semiclass. Opt., 5:S9, 2003.

[67] A.M. Rey, K. Burnett, R. Roth, M. Edwards, C.J. Williams, and C.W. Clark. Bogoliubov approach to superfluidity of atoms in an optical lattice. J. Phys. B: At. Mol. Opt. Phys., 36(5):825-841, 2003.

[68] D. van Oosten, P. van der Straten, and H. T. C. Stoof. Quantum phases in an optical lattice. Phys. Rev. A, 63(053601), 2001.

[69] P. S. Jessen and I. H. Deutsch. Optical Lattices. Advances in Atomic, Molecular and Optical Physics, 37, 1996.

[70] R. Grimm, M. Weidemuller, and Y. B. Ovchinnikov. Optical dipole traps for neutral atoms. Molecular and Optical Physics, 42:95, 2000.

[71] C. Orzel, A.K. Tuchman, M.L. Fenselau, M. Yasuda, and M.A. Kasevich. Squeezed States in a Bose-Einstein Condensate. Science, 291:2386, 2001.

[72] M. Greiner, O. Mandel, T. W. Hänsch, and I. Bloch. Collapse and revival of the matter wave field of a Bose-Einstein condensate. Nature, 419:51, 2002.

[73] D. van Oosten, P. van der Straten, and H.T.C. Stoof. Mott insulators in an optical lattice with high filling factors. arXiv:cond-mat/0205066, October 2002.

[74] O. Morsch, J.H. Müller, D. Ciampini, M. Cristiani, P.B. Blakie, C.J. Williams, P.S. Julienne, and E. Arimondo. Decay and revival of phase coherence of a Bose-Einstein condensate in a one-dimensional lattice. Phys. Rev. A, 67(3):031603, 2003.

[75] M.P.A. Fisher, P.B. Weichman, G. Grinstein, and D.S. Fisher. Boson localization and the superfluid-insulator transition. Phys. Rev. B, 40(15):546, July 1989.

[76] S. Sachdev. Quantum Phase Transitions. Cambridge University Press, 1999. 
[77] K. Burnett, M. Edwards, C.W. Clark, and M. Shotter. The Bogoliubov approach to number squeezing of atoms in an optical lattice. J. Phys. B: At. Mol. Opt. Phys., 35:1671, 2002.

[78] J.J. García-Ripoll, J.I. Cirac, P. Zoller, C. Kollath, U. Schollwöck, and J. von Delft. Variational ansatz for the superfluid Mott-insulator transition in optical lattices. arxiv:condmat/0306162, 2003.

[79] L. Amico and V. Penna. Dynamical Mean Field Theory of the Bose-Hubbard Model. Phys. Rev. Lett., 80(10):2189, 1998.

[80] L. Amico and V. Penna. Time-dependent mean-field theory of the superfluid-insulator phase transition. Phys. Rev. B, 62(2):1224, 2000.

[81] K. Sheshadri, H.R. Krishnamurthy, R. Pandit, and T.V. Ramakrishnan. Superfluid and Insulating Phases in an Interacting-Boson Model: Mean-Field Theory and the RPA. Europhys. Lett., 22(4):257, 1993.

[82] J.K. Freericks and H. Monien. Phase diagram of the Bose-Hubbard model. Europhys. Lett., 26(7):545, 1994.

[83] J.K. Freericks and H. Monien. Strong-coupling expansions for the pure and disordered Bose-Hubbard model. Phys. Rev. B, 53(5):2691, 1996.

[84] N. Elstner and H. Monien. Dynamics and thermodynamics of the Bose-Hubbard model. Phys. Rev. B, 59(19):12184, 1999.

[85] N. Elstner and H. Monien. A numerical exact solution of the Bose-Hubbard model. arXiv:cond-mat/9905367, 1999.

[86] R.T. Scalettar, G.G. Batrouni, and G.T. Zimanyi. Localization in Interacting, Disordered, Bose Systems. Phys. Rev. Lett., 66(24):3144, 1991.

[87] P. Niyaz, R.T. Scalettar, C.Y. Fong, and G.G. Batrouni. Phase transitions in an interacting boson model with near-neighbor repulsion. Phys. Rev. B, 50(1):362, 1994.

[88] G.G. Batrouni and R.T. Scalettar. World-line quantum Monte Carlo algorithm for a onedimensional Bose model. Phys. Rev. B, 46(14):9051, 1992.

[89] W. Krauth, N. Trivedi, and D. Ceperley. Superfluid-Insulator Transition in Disordered Boson Systems. Phys. Rev. Lett., 67(17):2307, 1991.

[90] A. van Otterlo and K.-H. Wagenblast. Coexistence of Diagonal and Off-Diagonal LongRange Order: A Monte Carlo Study. Phys. Rev. Lett., 72(22):3598, 1994.

[91] G.G. Batrouni, R.T. Scalettar, G.T. Zimanyi, and A.P. Kampf. Supersolids in the BoseHubbard Hamiltonian. Phys. Rev. Lett., 74(13):2527, 1995.

[92] I. Carusotto and Y. Castin. An exact reformulation of the Bose-Hubbard model in terms of a stochastic Gutzwiller ansatz. New Journal of Physics, 5:91.1, 2003. 
[93] S. R. White. Density Matrix Formulation for Quantum Renormalization Groups. Phys. Rev. Lett., 69(19):2863, 1992.

[94] R. V. Pai, R. Pandit, H.R. Krishnamurthy, and S. Ramasesha. One-dimensional Disordered Bose Hubbard Model: A Density-Matrix Renormalization Group Study. Phys. Rev. Lett., 76(16):2937, 1996.

[95] T.D. Kühner and H. Monien. Phases of the one-dimensional Bose-Hubbard model. Phys. Rev. B, 58(22):14741, 1998.

[96] S. Rapsch, U. Schollwöck, and W. Zwerger. Density matrix renormalization group for disordered bosons in one dimension. Europhys. Lett., 46:559, 1999.

[97] T. D. Kühner, S. R. White, and H. Monien. One-dimensional Bose-Hubbard model with nearest-neighbor interaction. Phys. Rev. B, 61(18):12474, 2000.

[98] S. Sachdev. Quantum Phase Transitions. Cambridge University Press, 1990, 2000.

[99] Z. Nazario and D. I. Santiago. Quantum States of Matter of Simple Bosonic Systems: BEC's, Superfluids and Quantum Solids. arXiv:cond-mat/0308005, August 2003.

[100] R. Roth and K. Burnett. Phase diagram of bosonic atoms in two-color superlattices. Phys. Rev. A, 68(2):023604, 2003.

[101] R. Roth and K. Burnett. Ultracold bosonic atoms in two-colour superlattices. Journal of Optics B: Quantum and Semiclassical Optics, 5(2):S50-S54, 2003.

[102] C.R. MacCluer. The Many Proofs and Applications of Perron's Theorem. Siam Review, 42(3):487, 2000.

[103] W.G. Unruh. Experimental Black-Hole Evaporation. Phys. Rev. Lett., 46:1351, 1981.

[104] S. E. Ch. Weinfurtner. Analog model for an expanding universe. arXiv:gr-qc/0404063, 2004.

[105] P. O. Fedichev and U. R. Fischer. Gibbons-Hawking Effect in the Sonic de Sitter SpaceTime of an Expanding Bose-Einstein-Condensed Gas. Phys. Rev. Lett., 91(24):240407, 2003.

[106] P. O. Fedichev and U. R. Fischer. Observer dependence for the phonon content of the sound field living on the effective curved space-time background of a Bose-Einstein condensate. Phys. Rev. D, 69(6):064021, 2004.

[107] P. O. Fedichev and U. R. Fischer. "Cosmological" quasiparticle production in harmonically trapped superfluid gases. Phys. Rev. A, 69(3):033602, 2004.

[108] M. Uhlmann and Yan Xu and R. Schutzhold. Aspects of cosmic inflation in expanding Bose-Einstein condensates. New Journal of Physics, 7, 2005. 
[109] C. Barcelo, S. Liberati, and M. Visser. Probing semiclassical analog gravity in BoseEinstein condensates with widely tunable interactions. Phys. Rev. A, 68(5):053613, 2003.

[110] R.A. Duine and H.T.C. Stoof. Quantum Evaporation of a Bose-Einstein Condensate. arXiv:cond-mat/0204529, 2002.

[111] U. R. Fischer. Quasiparticle universes in Bose-Einstein condensates. arXiv:condmat/0406086, 2004.

[112] S. Basak and P. Majumdar. 'Superresonance' from a rotating acoustic black hole. Classical and Quantum Gravity, 20:3907, 2003.

[113] T. R. Slatyer and C. M. Savage. Superradiant scattering from a hydrodynamic vortex. Classical and Quantum Gravity, 22(19):3833-3839, 2005.

[114] M. Novello and M. Visser and G. Volovik, editor. Artificial Black Holes. World Scientific, 2002.

[115] G. E. Volovik. The Universe in a Helium Droplet. Oxford University Press, 2003.

[116] G. E. Volovik. Superfluid analogies of cosmological phenomena. Physics Reports, 351(4):195-348, 2001.

[117] T. Jacobson and R. Parentani. An Echo of Black Holes. Scientific American, pages 69-75, 2005.

[118] M. Visser. Acoustic black holes: horizons, ergospheres and Hawking radiation. Classical and Quantum Gravity, 15(6):1767-1791, 1998.

[119] R. Balbinot, A. Fabbri, S. Fagnocchi, and R. Parentani. Hawking Radiation from Acoustic Black Holes, Short Distance and Back-Reaction Effects. arXiv.org:gr-qc/0601079, 2006.

[120] C. Barcelo and S. Liberati and M. Visser. Analogue gravity from Bose-Einstein condensates. Class. Quantum Grav., 18:1137, 2001.

[121] N.D. Birrell and P.C.W. Davies. Quantum fields in curved space. Cambridge University Press, 1982.

[122] J. F. Donoghue. General relativity as an effective field theory: The leading quantum corrections. Phys. Rev. D, 50(6):3874-3888, Sep 1994.

[123] S. Liberati, M. Visser, and S. Weinfurtner. Analogue quantum gravity phenomenology from a two-component Bose-Einstein condensate. Classical and Quantum Gravity, 23(9):3129_ 3154, 2006.

[124] R. Schützhold, G. Plunien, and G. Soff. Dielectric Black Hole Analogs. Phys. Rev. Lett., 88(6):061101, Jan 2002. 
[125] M. Visser. Hawking Radiation without Black Hole Entropy. Phys. Rev. Lett., 80(16):34363439, Apr 1998.

[126] C. Barceló, S. Liberati, and M. Visser. Analogue gravity from field theory normal modes? Classical and Quantum Gravity, 18(17):3595-3610, 2001.

[127] J.E. Lidsey. Cosmic dynamics of Bose-Einstein condensates. Classical and Quantum Gravity, 21(4):777-785, 2004.

[128] M. Cadoni. Acoustic analogues of two-dimensional black holes. Classical and Quantum Gravity, 22(2):409-419, 2005.

[129] C. Barceló, S. Liberati, S. Sonego, and M. Visser. Causal structure of analogue spacetimes. New Journal of Physics, 6:186, 2004.

[130] N. Bilic. Relativistic acoustic geometry. Classical and Quantum Gravity, 16(12):39533964, 1999.

[131] C. M. DeWitt and B. S. DeWitt. Black Holes. Gordon and Breach, New York, 1973. Proceedings of the 23rd Les Houches Summer School, Les Houches (France), August 1972.

[132] L. Parker. Particle Creation in Expanding Universes. Phys. Rev. Lett., 21(8):562-564, Aug 1968.

[133] L. Parker. Quantized Fields and Particle Creation in Expanding Universes. I. Phys. Rev., 183(5):1057-1068, Jul 1969.

[134] L. Parker. Quantized Fields and Particle Creation in Expanding Universes. II. Phys. Rev. D, 3(2):346-356, Jan 1971.

[135] C. Barcelo, S. Liberati, and M. Visser. Towards the Observation of Hawking Radiation in Bose-Einstein Condensates. International Journal of Modern Physics A, 18(21):37353745, 2003.

[136] Yu. Kagan, E. L. Surkov, and G. V. Shlyapnikov. Evolution of a Bose-condensed gas under variations of the confining potential. Phys. Rev. A, 54(3):R1753-R1756, Sep 1996.

[137] J. M. Vogels, C. C. Tsai, R. S. Freeland, S. J. J. M. F. Kokkelmans, B. J. Verhaar, and D. J. Heinzen. Prediction of Feshbach resonances in collisions of ultracold rubidium atoms. Phys. Rev. A, 56:1067, 1997.

[138] S. Inouye, M.R. Andrews, J. Stenger, H.-J. Miesner, D.M. Stamper-Kurn, and W. Ketterle. Observation of Feshbach resonances in a Bose-Einstein condensate. Nature, 392:151, 1998.

[139] E. A. Donley, N. R. Claussen, S. L. Cornish, J. L. Roberts, E. A. Cornell, and C. E. Wieman. Dynamics of collapsing and exploding Bose-Einstein condensates. Nature, 412(6844):295$299,2002$. 
[140] E. A. Calzetta and B. L. Hu. Bose-Einstein condensate collapse and dynamical squeezing of vacuum fluctuations. Phys. Rev. A), 68(4):043625, 2003.

[141] E. Calzetta and B. Hu. Early Universe Quantum Processes in BEC Collapse Experiments. International Journal of Theoretical Physics, 44(10):1691-1704, 2005.

[142] M. Visser, C. Barcelo, and S. Liberati. Analogue Models of and for Gravity. General Relativity and Gravitation, 34(10):1719-1734, October 2002.

[143] M. Sakagami and A. Ohashi. Hawking Radiation in Laboratories. Progress of Theoretical Physics, 107:1267, 2002.

[144] V. Cardoso. Acoustic black holes. arXiv.org:physics/0503042, 2005.

[145] H. Furuhashi, Y. Nambu, and H. Saida. Simulation of Acoustic Black Hole in a Laval Nozzle. arXiv.org:gr-qc/0601066, 2006.

[146] S. Giovanazzi, C. Farrell, T. Kiss, and U. Leonhardt. Conditions for one-dimensional supersonic flow of quantum gases. Phys. Rev. A, 70(6):063602, 2004. preprint, available at http://arxiv.org/abs/cond-mat/0405007.

[147] C. Farrell and U. Leonhardt. The perfectly matched layer in numerical simulations of nonlinear and matter waves. Journal of Optics B,7(1):1-4, 2005. preprint, available at http://arxiv.org/abs/cond-mat/0404020.

[148] S. Basak and P. Majumdar. 'Superresonance' from a rotating acoustic black hole. Classical and Quantum Gravity, 20(18):3907-3913, 2003.

[149] L. J. Garay and J.R. Anglin and J.I. Cirac and P. Zoller. Sonic black holes in dilute BoseEinstein condensates. Phys. Rev. A, 63:023611, 2001.

[150] T. Vachaspati. Propagating phase boundaries as sonic horizons. arXiv.org:gr-qc/0312069, 2003.

[151] T. A. Jacobson and G. E. Volovik. Event horizons and ergoregions in ${ }^{3} H e$. Phys. Rev. D, 58(6):064021, Aug 1998.

[152] T. Jacobson and G. Volovik. Effective spacetime and Hawking radiation from a moving domain wall in a thin film of ${ }^{3} \mathrm{He}$-A . JETP Letters, 68(11):874-880, 1998.

[153] G. E. Volovik. Horizons and Ergoregions in Superfluids. arXiv.org:gr-qc/0603093, 2006.

[154] G. E. Volovik. Momentum space topology of fermion zero modes on brane. JETP Letters, 75:55, 2002.

[155] G. E. Volovik. Black-hole horizon and metric singularity at the brane separating two sliding superfluids. JETP Letters, 76:240, 2002. 
[156] R. Schützhold and W.G. Unruh. Gravity wave analogues of black holes. Phys. Rev. D, 66(4):044019, Aug 2002.

[157] U. Leonhardt and P. Piwnicki. Relativistic Effects of Light in Moving Media with Extremely Low Group Velocity. Phys. Rev. Lett., 84(5):822-825, Jan 2000.

[158] U. Leonhardt and P. Piwnicki. Leonhardt and Piwnicki Reply:. Phys. Rev. Lett., 85(24):5253, Dec 2000.

[159] U. Leonhardt. A Primer to Slow Light. arXiv.org:gr-qc/0108085, 2001.

[160] W. G. Unruh and R. Schutzhold. On slow light as a black hole analogue. Phys. Rev. D, 68(2):024008, 2003.

[161] L. J. Garay, J. R. Anglin, J. I. Cirac, and P. Zoller. Sonic Analog of Gravitational Black Holes in Bose-Einstein Condensates. Phys. Rev. Lett., 85(22):4643-4647, Nov 2000.

[162] S. W. Hawking. Black hole explosions? Nature, 248(5443):30-31, 1974.

[163] S. Hawking. Particle creation by black holes. Communications in Mathematical Physics, 43(3):199-220, 1975.

[164] R. Parentani R. Brout, S. Massar and Ph. Spindel. A primer for black hole quantum physics. Physics Reports, 260(6):329-446, 1995.

[165] R. M. Wald. The Thermodynamics of Black Holes. Living Rev. Rel., 4(6), 2000.

[166] T. Jacobson. Introduction to Quantum Fields in Curved Spacetime and the Hawking Effect. arXiv:gr-qc/0308048, 2003.

[167] J. Traschen. An Introduction to Black Hole Evaporation. arXiv.org:gr-qc/0010055, 2000.

[168] M. K. Parikh and F. Wilczek. Hawking Radiation As Tunneling. Phys. Rev. Lett., 85(24):5042-5045, Dec 2000.

[169] J.D. Bekenstein. Black Holes and Entropy. Phys. Rev. D, 7(8):2333-2346, Apr 1973.

[170] M. Visser. Essential and Inessential Features of Hawking Radiation. International Journal of Modern Physics D, 12:649-661, 2003.

[171] A.D. Helfer. Do black holes radiate? Reports on Progress in Physics, 66(6):943-1008, 2003.

[172] T. Jacobson. Black-hole evaporation and ultrashort distances. Phys. Rev. D, 44(6):17311739, Sep 1991.

[173] W.G. Unruh. Sonic analogue of black holes and the effects of high frequencies on black hole evaporation. Phys. Rev. D, 51:2827, 1995. 
[174] R. Brout, S. Massar, R. Parentani, and Ph. Spindel. Hawking radiation without transPlanckian frequencies. Phys. Rev. D, 52(8):4559-4568, Oct 1995.

[175] S. Corley and T. Jacobson. Hawking spectrum and high frequency dispersion. Phys. Rev. D, 54(2):1568-1586, Jul 1996.

[176] B. Reznik. Trans-Planckian tail in a theory with a cutoff. Phys. Rev. D, 55(4):2152-2158, Feb 1997.

[177] S. Corley. Computing the spectrum of black hole radiation in the presence of high frequency dispersion: An analytical approach. Phys. Rev. D, 57(10):6280-6291, May 1998.

[178] S. Corley and T. Jacobson. Lattice black holes. Phys. Rev. D, 57(10):6269-6279, May 1998.

[179] T. Jacobson and D. Mattingly. Hawking radiation on a falling lattice. Phys. Rev. D, 61(2):024017, Dec 1999.

[180] W. G. Unruh and R. Schutzhold. Universality of the Hawking effect. Phys. Rev. D, 71(2):024028, 2005.

[181] T. A. Jacobson and R. Parentani. An Echo of Black Holes. Scientific American Magazine, December 2005.

[182] T. Jacobson. On the origin of the outgoing black hole modes. Phys. Rev. D, 53(12):70827088, Jun 1996.

[183] S. Massar. Semiclassical back reaction to black hole evaporation. Phys. Rev. D, 52(10):5857-5864, Nov 1995.

[184] R. M. Wald. Quantum Field Theory in Curved Spacetime and Black Hole Thermodynamics. University of Chicago Press, 1994.

[185] R. Balbinot, S. Fagnocchi, A. Fabbri, and G. P. Procopio. Backreaction in Acoustic Black Holes. Phys. Rev. Lett., 94(16):161302, 2005.

[186] R. Schutzhold, M. Uhlmann, Y. Xu, and U. R. Fischer. Quantum backreaction in dilute Bose-Einstein condensates. Phys. Rev. D, 72:105005, 2005.

[187] S. Sinha, A. Raval, and B. Hu. Black Hole Fluctuations and Backreaction in Stochastic Gravity. Foundations of Physics, 33(1):37-64, 2003.

[188] S. Liberati, S. Sonego, and M. Visser. Unexpectedly large surface gravities for acoustic horizons? Classical and Quantum Gravity, 17:2903, 2000.

[189] S.A. Fulling. Aspects of Quantum Field Theory in Curved Space-Time. Cambridge University Press, 1989.

[190] G.F.R. Ellis and T. Rothman. Lost horizons. Am. J. Phys., 61(10):883-893, Oct 1993. 
[191] J. Audretsch. Cosmological particle creation as above-barrier reflection: approximation method and applications. J. Phys. A: Math. Gen., 12(8):1189-1203, 1979.

[192] L. P. Grishchuk and Y. V. Sidorov. Squeezed quantum states of relic gravitons and primordial density fluctuations. Phys. Rev. D, 42(10):3413-3421, Nov 1990.

[193] S. Winitzki. Cosmological particle production and the precision of the WKB approximation. Phys. Rev. D, 72(10):104011, 2005.

[194] U. R. Fischer and R. Schutzhold. Quantum simulation of cosmic inflation in two-component Bose-Einstein condensates. Phys. Rev. A, 70(6):063615, 2004.

[195] D. Mattingly. Modern tests of Lorentz invariance. Living Rev. Rel., 8:5, 2005.

[196] J. Martin and R.H. Brandenberger. Trans-Planckian problem of inflationary cosmology. Phys. Rev. D, 63(12):123501, May 2001.

[197] A.A. Abrikosov and L.P. Gorkov and I.E. Dzyaloshinski. Methods of Quantum Field Theory in Statistical Mechanics. Prentice-Hall Inc., 1963.

[198] C. Bernard and A. Duncan. Regularization and renormalization of quantum field theory in curved space-time. Annals of Physics, 107:201, 1977.

[199] A. H. Guth. Inflationary universe: A possible solution to the horizon and flatness problems. Phys. Rev. D, 23(2):347-356, Jan 1981.

[200] G.W. Gibbons and S.W. Hawking. Cosmological event horizons, thermodynamics, and particle creation. Phys. Rev. D, 15(10):2738, May 1977.

[201] A.S. Lapedes. Bogoliubov transformations, propagators, and the Hawking effect. J. Math. Phys., 19(11):2289, November 1978.

[202] M. Abramowitz and I. A. Stegun. Handbook of Mathematical Functions with Formulas, Graphs, and Mathematical Tables. Dover, New York, ninth dover printing, tenth gpo printing edition, 1964.

[203] A. R. Liddle and D. H. Lyth. Cosmological Inflation and Large-Scale Structure. Cambridge University Press, 2000.

[204] S. Weinfurtner and M. Visser, 2006. Private Communication.

[205] P.J. Steinhardt and N. Turok. Cosmic evolution in a cyclic universe. Phys. Rev. D, 65(12):126003, May 2002.

[206] E. M. Lifshitz and L. D. Landau. Mechanics. Elsevier, 3rd edition, 1976.

[207] J.J. García-Ripoll, V.M. Pérez-García, and P. Torres. Extended Parametric Resonances in Nonlinear Schrödinger Systems. Phys. Rev. Lett., 83(9):1715-1718, Aug 1999. 
[208] M. Modugno, C. Tozzo, and F. Dalfovo. Detecting phonons and persistent currents in toroidal Bose-Einstein condensates by means of pattern formation. arXiv.org:condmat/0605183, 2006.

[209] I. Zlatev, G. Huey, and P.J. Steinhardt. Parametric resonance in an expanding universe. Phys. Rev. D, 57(4):2152-2157, Feb 1998.

[210] A. A. Norrie. A Classical Field Treatment of Colliding Bose-Einstein Condensates. PhD thesis, University of Otago, 2005.

[211] B. M. Caradoc-Davies. Vortex Dynamics in Bose-Einstein Condensates. PhD thesis, University of Otago, 2000.

[212] J. L. Roberts, N. R. Claussen, James P. Burke, Chris H. Greene, E. A. Cornell, and C. E. Wieman. Resonant Magnetic Field Control of Elastic Scattering in Cold ${ }^{85}$ Rb. Phys. Rev. Lett., 81(23):5109-5112, Dec 1998.

[213] C.W. Gardiner. Quantum Matter Waves. Private Communication, 2005.

[214] M. Brewczyk and P. Borowski and M. Gajda and K. Rzazewski. Temperature-dependent Bogoliubov approximation in the classical field approach to weakly interacting Bose gases. J. Phys. B: At. Mol. Opt. Phys., 37:2725, 2004.

[215] M. Edwards and R.J. Dodd and C.W. Clark and K. Burnett. Zero-Temperature, Mean-Field Theory of Atomic Bose-Einstein Condensates. J. Res. Natl. Inst. Stand. Technol., 101:553, 1996.

[216] A. Griffin. Theory of excitations of the condensate and non-condensate at finite temperatures. arXiv:cond-mat/9901172, 1999.

[217] W. Hansel and P. Hommelhoff and T. W. Hansch and J. Reichel. Bose-Einstein condensation on a microelectronic chip. Nature, 413:498, 2001. (Experimental: optical box trap).

[218] T. P. Meyrath and F. Schreck and J. L. Hanssen and C.-S. Chuu and M. G. Raizen. BoseEinstein condensate in a box. Phys. Rev. A, 71(4):041604, 2005.

[219] Y. Zhang and L. Yin. Three-body recombination of a condensed Bose gas near a Feshbach resonance. Phys. Rev. A, 72(4):043607, 2005.

[220] E. Braaten and H.-W. Hammer and M. Kusunoki. Efimov States in a Bose-Einstein Condensate near a Feshbach Resonance. Phys. Rev. Lett., 90(17):170402, 2003.

[221] N. R. Claussen and E. A. Donley and S. T. Thompson and C. E. Wieman. Microscopic Dynamics in a Strongly Interacting Bose-Einstein Condensate. Phys. Rev. Lett., 89(1):010401, Jun 2002.

[222] R. A. Duine and H. T. C. Stoof. Dynamics of a Bose-Einstein condensate near a Feshbach resonance. Phys. Rev. A, 68(1):013602, 2003. 
[223] A. A. Norrie and R. J. Ballagh and C. W. Gardiner and A. S. Bradley. Three-body recombination of ultracold Bose gases using the truncated Wigner method. Phys. Rev. A, 73(4):043618, April 2006.

[224] M. Visser. Acoustic propagation in fluids: an unexpected example of Lorentzian geometry. arXiv.org:gr-qc/9311028, 1993.

[225] U. Leonhardt and T. Kiss and P. Ohberg. Bogoliubov theory of the Hawking effect in BoseEinstein condensates. J. Opt. B: Quantum Semiclass Opt., 5:S42, 2003.

[226] A. L. Fetter. Ground state and excited states of a confined condensed Bose gas. Phys. Rev. A, 53(6):4245-4249, Jun 1996.

[227] L. D. Landau and E. M. Lifshitz. Fluid Mechanics. Pergamon Press, 1959,1963.

[228] Chia-Shun Yih. Fluid Mechanics. West River Press, 1977,1979.

[229] P.B. Blakie. Optical Manipulation of Bose-Einstein Condensates. PhD thesis, University of Otago, July 2001.

[230] J. Brand and W. P. Reinhardt. Generating ring currents, solitons and svortices by stirring a Bose-Einstein condensate in a toroidal trap. J. Phys. B: At. Mol. Opt. Phys., 34(4):L113L119, 2001.

[231] J. A. Sauer, M. D. Barrett, and M. S. Chapman. Storage Ring for Neutral Atoms. Phys. Rev. Lett., 87(27):270401, Dec 2001.

[232] S. Gupta, K. W. Murch, K. L. Moore, T. P. Purdy, and D. M. Stamper-Kurn. Bose-Einstein Condensation in a Circular Waveguide. Phys. Rev. Lett., 95(14):143201, 2005.

[233] M. B. Crookston, P. M. Baker, and M. P. Robinson. A microchip ring trap for cold atoms. J. Phys. B: At. Mol. Opt. Phys., 38(18):3289-3298, 2005.

[234] A. S. Arnold, C. S. Garvie, and E. Riis. Large magnetic storage ring for Bose-Einstein condensates. Phys. Rev. A, 73(4):041606, 2006.

[235] B. G. Swingle and T. A. B. Kennedy. Generation of topological flows by phase imprinting. J. Phys. B: At. Mol. Opt. Phys., 38(19):3503-3515, 2005.

[236] R. Schutzhold. On the detectability of quantum radiation in Bose-Einstein condensates. arXiv.org:quant-ph/0602180, 2006.

[237] Y. Castin. Course 1: Bose-Einstein Condensates in Atomic Gases: Simple Theoretical Results. Les Houches Summer School, 72:1, 2001. preprint, available at http://arxiv.org/abs/cond-mat/0105058.

[238] S. A. Morgan, S. Choi, K. Burnett, and M. Edwards. Nonlinear mixing of quasiparticles in an inhomogeneous Bose condensate. Phys. Rev. A, 57(5):3818-3829, May 1998. 
[239] E.M. Lifshitz and L.P. Pitaevskii. Statistical Physics, Part 2. Pergamon, Oxford, 1980.

[240] A. L. Fetter. Theory of a dilute low-temperature trapped Bose condensate. arXiv:condmat/9811366, 1998.

[241] J. Javanainen and Y. Zheng. Perturbing a persistent current with an external potential. Phys. Rev. A, 63(6):063610, May 2001.

[242] P. Villain and M. Lewenstein. Fermi-Pasta-Ulam problem revisited with a Bose-Einstein condensate. Phys. Rev. A, 62(4):043601, Sep 2000.

[243] J. D. Bekenstein and V. F. Mukhanov. Spectroscopy of the quantum black hole. Physics Letters B, 360:7, 1995.

[244] S. Corley and T. Jacobson. Black hole lasers. Phys. Rev. D, 59(12):124011, May 1999.

[245] C. Barcelo, A. Cano, L. J. Garay, and G. Jannes. Stability analysis of sonic horizons in Bose-Einstein condensates. Phys. Rev. D, 74(2):024008, 2006. preprint, available at http://arxiv.org/abs/gr-qc/0603089.

[246] W. H. Press, B. P. Flannery, S. A. Teukolsky, and W. T. Vetterling. Numerical Recipes: The Art of Scientific Computing. Cambridge University Press, Cambridge (UK) and New York, 2nd edition, 1992.

[247] M. Frigo and S. G. Johnson. The Design and Implementation of FFTW3. Proceedings of the IEEE, 93(2):216-231, 2005. special issue on "Program Generation, Optimization, and Platform Adaptation". 